\title{
MedienPädagogik
}

Zeitschrift für Theorie und Praxis der Medienbildung

Lernsituationen mit Metaphern und Wikibooks.

Fallstudien zu Entwicklungspotenzialen einer integrativen Medienbildung in der Lehrerinnen- und Lehrerbildung

\section{Problematisierung der Praxis und Forschungsstand}

Franco Rau

\section{Zusammenfassung}

Im dritten Teil dieser Arbeit erfolgt eine Problematisierung der Praxis sowie die Darstellung des Forschungsstandes für zwei ausgewählte Schwerpunkte. Der erste Schwerpunkt fokussiert den Stand der Medienbildung im Lehramtsstudium (Kap. 4). Der zweite Schwerpunkt widmet sich den erziehungswissenschaftlichen Studienanteilen als Handlungsund Forschungsfeld des vorliegenden Projektes (Kap. 5). In vergleichender Betrachtung bildungspolitischer Leitbilder und der aktuellen Praxis werden jeweils konkrete Probleme benannt und potenzielle Gestaltungsspielräume markiert (Kap. 4.2, 5.2). Zur Vorbereitung eines theoretisch und empirisch begründeten Entwurfs von Lernsituationen zur integrativen Medienbildung erfolgt zum einen eine theoretisch-begriffliche Verortung medienpädagogischer Begriffe (Kap. 4.3) sowie eine Betrachtung des aktuellen Forschungsund Diskussionsstandes zu den Themenfeldern «soziale Medien als Mittel zur Gestaltung von Hochschullehre» (Kap. 4.4) und "soziale Medien als Gegenstand universitärer Medienbildung» (Kap. 4.5). Zum anderen erfolgt zur Berücksichtigung des Handlungs- und Forschungsfeldes eine Betrachtung von theoretischen und empirischen Ansätzen zur Erfahrung-Theorie-Relationierung im Rahmen der erziehungswissenschaftlichen Studienanteile (Kap. 5.3). Es werden die Potenziale von Metaphern als Forschungskonstrukt und Reflexionsmöglichkeit in der Lehrerinnen- und Lehrerbildung (Kap. 5.4) sowie in weiteren pädagogischen Kontexten (Kap. 5.5) vorgestellt und hinsichtlich ihrer Relevanz für die vorliegende Arbeit diskutiert.

\section{Medienbildung mit sozialen Medien im Lehramtsstudium}

\subsection{Methodische Verortung}

Die Problematisierung der Praxis bildet gemäss des Prozessmodells der entwicklungsorientierten Bildungsforschung (Abb. 4.1) von Sesink und Reinmann (2015) einen zentralen Ausgangspunkt für das vorliegende Forschungs- und Entwicklungsprojekt (Kap. 3.1.1). Die Problematisierung bzw. das Erkennen eines Problems umfasst für Sesink und Reinmann $(2015,72)$ das Hinterfragen des Verhältnisses von realer Praxis und normativer Orientierung sowie der zwei zugehörigen Pole. Im Fokus der ersten Problematisierung steht die (fehlende) Medienbildung im Lehramtsstudium (Kap. 4.2). In Betrachtung bildungspolitischer Leitbilder zur Medienbildung wird im 
Vergleich mit der aktuellen Praxis der Lehrerinnen- und Lehrerbildung an der TU Darmstadt gezeigt, «dass «etwas nicht stimmt»» (Sesink und Reinmann 2015, 71). Die aktuelle Praxis muss als problematisch eingeschätzt werden, da nicht alle Lehramtsstudierenden im Rahmen des Lehramtsstudiums angemessen auf die Aufgaben einer schulischen Medienbildung vorbereitet werden (Kap. 4.2). Die Problematisierung erfolgt weitgehend auf einer makrodidaktischen Ebene und fokussiert den Aufbau aktueller Studiengänge und Module. Die normativen Leitbilder werden in Form verschiedener bildungspolitischer Positionen und Strategiepapiere untersucht. Die konkrete Praxis der TU Darmstadt wird über die Analyse von Modulhandbüchern und Projekterfahrungen im Lehramtsstudium der TU Darmstadt diskutiert (Grell et al. 2015).

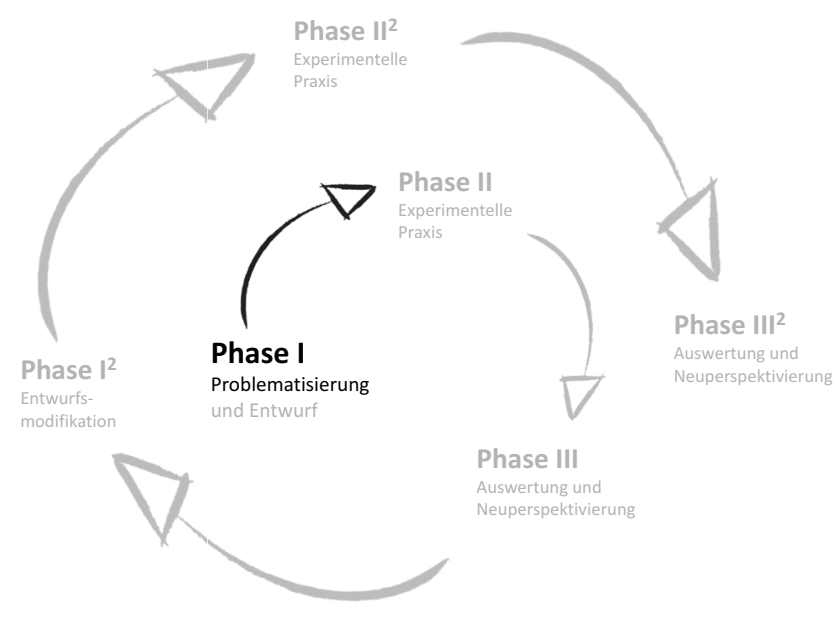

Abb. 4.1: Verortung des Kapitels im Phasenmodell der entwicklungsorientierten Bildungsforschung.

Die vorgenommene Problematisierung bildet den Ausgangspunkt zum Entwurf einer neuen Praxis. Zur Vorbereitung eines theoretisch begründeten Entwurfs erfolgt in Adaption der von Sesink und Reinmann (2015) skizzierten Forschungsphase die Aufarbeitung des aktuellen Forschungsstandes. In Anlehnung an die Empfehlungen von Tulodziecki et al. $(2013,2014)$ werden theoretisch und empirisch fundierte Erkenntnisse präsentiert und für die Projektziele diskutiert. Im Rahmen einer theoretisch-begrifflichen Klärung erfolgt $u$. a. eine vertiefende Auseinandersetzung mit dem Konstrukt der medienpädagogischen Kompetenz als Orientierungshilfe zur Formulierung angemessener Zielstellungen für die Praxis (Kap. 4.3.2). Darüber hinaus wird auf Basis verschiedener Literaturübersichten und Fallstudien eine Synthese von Erkenntnissen zu sozialen Medien als Mittel zur Gestaltung von Hochschullehre vorgenommen (Kap. 4.4). Weiterhin werden mediendidaktische Projekte sowie Ergebnisse aus dem E-Learning-2.0-Diskurs diskutiert. Diese Erkenntnisse und Ansätze 
dienen u. a. als mediendidaktische Grundlage für Entscheidungen zum Einsatz digitaler sozialer Medien im Rahmen des Konzeptes (Kap. 6). Darüber hinaus werden im Rahmen des Entwurfs aktuelle Konzepte und Diskussionsthemen aus einer überwiegend deutschsprachigen medienerzieherischen Perspektive berücksichtigt (Kap. 4.5). Aus der Kombination dieser Perspektiven können soziale Medien als «Mittel» und «Gegenstand» universitärer Medienbildung verstanden werden.

In Anlehnung an die skizzierten Prozess- und Qualitätsstandards (Kap. 2.2) erfüllt die vorgenommene Verknüpfung einer praxisorientierten Problematisierung sowie der Erarbeitung des aktuellen Forschungsstandes zwei Funktionen. Zum einen wurde das Ziel verfolgt, über die Berücksichtigung einer praxis- und forschungsorientierten Perspektive dem Qualitätskriterium zur Berücksichtigung der «Praxisrelevanz von Forschungsfragen und Praxistauglichkeit von Lösungen» (Tulodziecki et al. 2013, 228) gerecht zu werden. Zum anderen wurde durch die zwei berücksichtigten Perspektiven das Kriterium zum «Zusammenwirken von Forschung und Praxis» (Tulodziecki et al. 2013, 229) berücksichtigt. Wenngleich im Rahmen des Projektes die Rolle des Lehrenden und des Forschenden durch die gleiche Person realisiert wurde, eröffnete die Unterscheidung zwischen Praxisproblematisierung und Erarbeitung von Forschungserkenntnissen einen - wie auch von Tulodziecki et al. (2013) vorgeschlagenen - temporären Perspektivwechsel im Rahmen der Vorbereitung des Projektes.

\subsection{Problematisierung der Medienbildung im Lehramtsstudium}

Die Problematisierung der Medienbildung im Lehramtsstudium erfolgt in Betrachtung des Verhältnisses von normativen Orientierungen bzw. vorhandenen Leitbildern und der Praxis an der TU Darmstadt. Die Diskussion aktueller Leitbilder erfolgt im ersten Schritt in Betrachtung verschiedener bildungspolitischer Dokumente und Berichte (Kap. 4.2.1). Auf Basis von drei skizzierten Leitideen (Kap. 4.2.1.1, 4.2.1.2, 4.2.1.3) werden Implikationen und Herausforderungen für die Lehrerinnen- und Lehrerbildung markiert (Kap. 4.2.1.4). Im zweiten Schritt erfolgt eine Diskussion der realen Praxis der Medienbildung im Lehramtsstudium der TU Darmstadt (Kap. 4.2.2). Dafür werden zwei zentrale Problemdimensionen unterschieden: «Begrenzte Möglichkeiten zum Lernen mit digitalen Medien» (Kap. 4.2.2.1) sowie «Lernen über Medien nur als Wahloption» (Kap. 4.2.2.2). Zum Abschluss der Problematisierung werden potenzielle Handlungsstrategien zum Umgang mit dem Spannungsfeld zwischen normativen Ansprüchen einer Medienbildung im Lehramtsstudium einerseits und der aktuellen Praxis an der TU Darmstadt anderseits diskutiert (Kap. 4.2.3). 


\subsubsection{Medienbildung als bildungspolitisches Leitbild}

Zur Identifikation relevanter Leitbilder erfolgte eine Onlinerecherche bildungspolitischer Dokumente ${ }^{69}$. Zur Recherche wurden die länderspezifischen Bildungsserver und Schulportale sowie die Website der Initiative «Keine Bildung ohne Medien» (KBoM) herangezogen ${ }^{70}$. In einem weiten Begriffsverständnis umfassen bildungspolitische Dokumente u. a. Forderungen und Positionspapiere von Initiativen (z. B. KBoM 2009, 2011), vom Bund und Ländern initiierte Expertenberichte und -empfehlungen (z. B. BMBF 2010; BMFSFJ 2013) sowie Beschlüsse und Strategiepapiere der Kultusministerkonferenz (z. B. KMK 2012, 2016a). Auf Basis der Recherche werden im Folgenden drei konsensuale normative Orientierungen zur Medienbildung skizziert: (1.) «Medienbildung als Bildungs- und Erziehungsauftrag» (Kap. 4.2.1.1), (2.) «Lernen mit und über (digitale) Medien als Leitidee» (Kap. 4.2.1.2) sowie (3.) «Integrativer Ansatz statt eigener Lernbereich» (Kap. 4.2.1.3). Im Zusammenhang mit den skizzierten Zielstellungen für die schulische Medienbildung werden auch vielfältige Herausforderungen und Anforderungen für die Lehrerinnen- und Lehrerbildung diskutiert. Die zentralen Implikationen und Herausforderungen für die Lehrerinnen- und Lehrerbildung (Kap. 4.2.1.4) werden zum Abschluss des Kapitels präzisiert.

\subsubsection{Medienbildung als Bildungs- und Erziehungsauftrag}

Medienbildung wird in zahlreichen bildungspolitischen Dokumenten als relevanter staatlicher Erziehungs- und Bildungsauftrag markiert (z. B. BMBF 2010; LKM 2015; KMK 2016a). Die Beschreibung von Medienkompetenz als neue Kulturtechnik (z. B. KMK 2012; LKM 2015) unterstreicht die Relevanz von Medienbildung in bildungspolitischen und -administrativen Kontexten. Der Begriff «Medienbildung» lässt sich in diesem Zusammenhang als «breitbandiges Konzept» zur pädagogisch strukturierten Medienkompetenzvermittlung verstehen (Jörissen 2011, 214). Dieses Begriffsverständnis bestätigt sich sowohl bei Betrachtung älterer Veröffentlichungen wie dem Expertenbericht des BMBF (2010) als auch in jüngeren bildungspolitischen und -administrativen Dokumenten. Im Positionspapier der Länderkonferenz Medienbildung

69 Im ersten Schritt der Recherche wurde die Website der Initiative «Keine Bildung ohne Medien!» als Startpunkt gewählt. Die Initiative versteht sich selbst als «Netzwerk, das engagierte Einzelpersonen und Einrichtungen im Feld der Medienbildung zusammenführt», als «Impulsgeber für bildungspolitische Aktivitäten zur Förderung von Medienbildung» sowie als «Anlaufstelle für das Anstoßen regionaler und überregionaler Aktivitäten». Mit der «Länderkarte Medienkompetenzförderung» bietet die Website einen strukturierten Überblick über bildungspolitische sowie bildungsadministrative Veröffentlichungen auf Bundes- und Landesebene. Zudem wurde die Suchoption der Webseite verwendet.

70 Die heterogene Struktur sowie die unterschiedlichen Begriffe einzelner Websites stellten eine Schwierigkeit für ein standardisiertes Recherchevorgehen dar. Während zum Zeitpunkt der Recherche der Hessische Bildungsserver den Begriff «Medienbildung» verwendete, nutzte das Schulportal Thüringen die Begriffskombination «Medien und Bildung». Zur Identifikation relevanter Dokumente wurden jeweils die Themenseiten sowie die Suchoptionen verwendet. Als Suchbegriffe dienten u. a. «Medienbildung», «Medienkompetenz» sowie «integrative Medienbildung». Es wurden nur Veröffentlichungen mit Bezug auf die Institutionen Schulen und Hochschule berücksichtigt. 
(LKM 2015) wird Medienkompetenz z. B. zur «unverzichtbaren Kulturtechnik» erklärt und deren Erwerb soll «durch eine verbindliche, grundlegende und systematische schulische Medienbildung» (ebd., 3) erfolgen. In einem Koalitionsantrag (Bundestag 2015) werden neben dem Medienkompetenzbegriff auch die Formulierungen «digitale Bildung» (ebd., 3) und «digitale Medienkompetenz verwendet» (ebd., 5). Um eine «eine breite und vertiefte und damit nachhaltige Medienbildung zu erzielen» (Bundestag 2015, 2), werden verschiedene Handlungsfelder und Aufgaben markiert. In einer vergleichbaren Logik kann auch das Strategiepapier der KMK (2016a) verstanden werden. Wenngleich in dem Strategiepapier zunächst die Formulierung «Kompetenzen in der digitalen Welt» verwendet wird (KMK 2016a), werden vor allem Ansätze zur pädagogisch strukturierten Kompetenzvermittlung diskutiert. Inwiefern die Fokussierung digitaler Aspekte, wie von der KMK (2016a) formuliert, dabei «die bisher entwickelten Konzepte zur Medienbildung» (ebd., 14) erweitert oder einschränkt, wird durchaus kontrovers diskutiert ${ }^{71}$. Wenngleich sich das Verständnis von Medienkompetenz bzw. "Kompetenzen in der digitalen Welt» in den Papieren unterscheidet, ist hinsichtlich der zwei folgenden Aspekte ein Konsens erkennbar:

1. Die Relevanz bzw. Notwendigkeit einer pädagogisch strukturierten Medienkompetenzvermittlung als staatlicher Bildungs- und Erziehungsauftrag sowie der Bezeichnung von Medienkompetenz als Kulturtechnik.

2. Der Einforderung einer strukturellen Verankerung von Medienbildungsangeboten in formalen Bildungsinstitutionen, insbesondere in der Schule.

Verschiedene medienpädagogische Initiativen, wie z. B. KBoM (2009, 2011, 2014), betonen im Rahmen ihrer bildungspolitischen Forderungen die Bedeutung von Medienbildung und Medienkompetenz. Entsprechende Forderungen finden sich in den letzten Jahren aber auch zunehmend in den Publikationen des Bundesministeriums für Bildung und Forschung (BMBF 2010, 2016) sowie der KMK (2012, 2016a). Im Vorwort des Expertenberichtes des BMBF (2010) markiert Schavan beispielsweise, dass eine «grundlegende Medienbildung» dazu beitragen kann, sich in einer «digitalen Welt» zurechtzufinden. Dafür «Orientierung zu vermitteln», so Schavan weiter, «ist ein staatlicher Bildungsauftrag». Im aktuellen Strategiepapier der KMK (2016a) wird auf den Beschluss zur «Medienbildung in der Schule» (KMK 2012) verwiesen, welcher weiterhin gültig ist (KMK 2016a, 10 f.). Zudem mache es die «digitale Revolution»» erforderlich, so die KMK (2016a), «nunmehr verbindliche Anforderungen zu formulieren, über welche Kenntnisse, Kompetenzen und Fähigkeiten Schülerinnen und Schüler am Ende ihrer Pflichtschulzeit verfügen sollen, damit sie zu einem selbstständigen

71 Beispielsweise kritisiert die Gesellschaft für Informatik (GI 2016) den reduzierten Bildungsbegriff im Entwurf KMK (2016a) und fordert u. a. die Einführung eines eigenen Fachs. Auch die Gesellschaft für Medienpädagogik und Kommunikationskultur (GMK 2016) problematisiert u. a. die verwendeten Begriffe im Entwurf der KMK (2016a) und hinterfragt das Verhältnis zum früheren Beschluss «Medienbildung in der Schule» der KMK (2012). 
und mündigen Leben in einer digitalen Welt befähigt werden» (ebd., 11). Auf Basis der exemplarischen Belege wird davon ausgegangen, dass Medienbildung - verstanden als pädagogisch strukturierte Medienkompetenzvermittlung - im bildungspolitischen Diskurs als staatlicher Bildungs- und Erziehungsauftrag betrachtet wird.

Zur Realisierung dieses Auftrages wird in verschiedenen Positionspapieren von bildungspolitischen Initiativen KBoM (z. B. 2009, 2011, 2014), dem BMBF (z. B. 2010, 2016) dem Bundesministerium für Familie, Senioren, Frauen und Jugend (BMFSFJ 2013) und der KMK $(2012,2016 a)$ eine strukturelle Verankerung von Medienbildungsangeboten in formalen Bildungsinstitutionen empfohlen bzw. gefordert. Als gemeinsamer Fokus dieser Forderungen steht dabei die Institution Schule bzw. der Schulunterricht ${ }^{72}$. Dabei finden sich Formulierungen, welche die Institution Schule als relevantes Handlungsfeld charakterisieren. So sieht das BMBF $(2016,2)$ das Bildungssystem in der Verantwortung, «die strukturellen Möglichkeiten zu schaffen, um Heranwachsenden den kompetenten Umgang mit digitalen Medien zu vermitteln». In diesem Kontext heben sie auch die Verantwortung bestimmter Schulfächer hervor und sehen relevante Aufgaben «in den Lehrplänen der Länder, in der Ausbildung der Lehrkräfte und auch in der infrastrukturellen Ausstattung von Schulen» (BMBF 2016, 3). Die «systematische Einbettung von Medienbildung und Medienkompetenzvermittlung in das Unterrichts-, aber auch in das Schulgeschehen» ist auch für das $\operatorname{BMFSFJ}(2013,14)$ «weiter ein Entwicklungsfeld». Mit mehr Nachdruck und einer stärkeren Betonung der Verbindlichkeit dieser Aufgabe fordert die KBoM (2011):

«In den Orientierungs- und Rahmenplänen für frühkindliche Bildungseinrichtungen sowie in den Curricula und Prüfungen aller Schularten sind medienpädagogische Themen verbindlich zu verankern. Bundesweit sollten im Bereich Medienkompetenz klare Mindeststandards für die einzelnen Altersstufen festgelegt werden: Kein Jugendlicher darf die Schule ohne grundlegende Medienbildung verlassen» KBoM $(2011,5)$.

Eine Betonung der Verbindlichkeit schulischer Medienbildung kann auch mit Bezug auf jüngere Publikationen veranschaulicht werden. So soll mit dem Beschluss der KMK (2012) «Medienbildung in der Schule» ein Beitrag geleistet werden, um «Medienbildung als Pflichtaufgabe schulischer Bildung nachhaltig zu verankern sowie den Schulen und Lehrkräften Orientierung für die Medienbildung in Erziehung und Unterricht zu geben» (ebd., 3). Auch im Positionspapier der LKM $(2015,2)$ wird eine

72 Die Forderung der Initiative $\operatorname{KBoM}(2009,2011,2014)$ sowie des BMFSFJ (2013) sind nicht beschränkt auf die Institution der Schule. Um allen «Mitgliedern der Gesellschaft eine grundlegende Medienbildung zu ermöglichen», forderte beispielsweise die Initiative «KBoM!» im Jahr 2011, «müssen medienpädagogische Angebote und Programme in allen Bildungsbereichen und allen Erziehungs- und Bildungseinrichtungen strukturell verankert werden» (KBoM 2011, 5). Im Beschluss der KMK (2012) heisst es dazu: «Die Entwicklung von umfassender Medienkompetenz durch Medienbildung ist eine gesamtgesellschaftliche Aufgabe, die nur im Zusammenwirken von Schule und Elternhaus sowie mit den Verantwortlichen in Politik, Wirtschaft und Kultur bewältigt werden kann» (KMK 2012, 3). 
«verbindliche, grundlegende und systematische schulische Medienbildung» zur Förderung von Medienkompetenz gefordert. Die Verbindlichkeit einer Medienbildung hinsichtlich einer strukturellen Verankerung wird, wie von der KBoM (2011) gefordert, über die Integration relevanter Inhalte und Ziele in Lehr- und Bildungsplänen diskutiert und aktuell angestrebt. Mit der Zielstellung, «eine breite und vertiefte und damit nachhaltige Medienbildung zu erzielen», heisst es beispielsweise im Antrag der Fraktionen der CDU/CSU und SPD (Bundestag 2015), dass «digitale Inhalte und die Zielsetzung der digitalen Selbstständigkeit fächerübergreifend und verpflichtend in den Bildungsplänen aller Schulstufen verankert werden [müssen]» (Bundestag 2015, 2). Zudem haben sich die Kultusministerien der Bundesländer gemäss der aktuellen KMK-Strategie dazu verpflichtet, «dafür Sorge zu tragen, dass alle Schülerinnen und Schüler, die zum Schuljahr 2018/2019 in die Grundschule eingeschult werden oder in die Sek I eintreten, bis zum Ende der Pflichtschulzeit die in diesem Rahmen formulierten Kompetenzen erwerben können» (ebd., 18). Zur Realisierung dieser Aufgabe sind gemäss der KMK (2016a, 22) «neben möglichen Anpassungen der länderspezifischen Lehr- und Bildungspläne auch niederschwellige Maßnahmen wie die Einführung/Anpassung von Medienpässen/Computerführerscheinen oder ergänzende Erlasse zu den curricularen Vorgaben möglich». Wenngleich die von der KMK (2016a) formulierte Selbstverpflichtung, für Medienbildung «Sorge zu tragen», im Vergleich zur Forderung der KBoM (2011) eher zurückhaltend erscheint, markieren die exemplarischen Belege auf Bundesebene die Relevanz einer pädagogisch strukturierten Medienkompetenzvermittlung in der bildungspolitischen Debatte.

\subsubsection{Lernen mit und über (digitale) Medien als Leitidee}

Das Verständnis von Medienbildung bleibt in bildungspolitischen und -administrativen Kontexten nicht auf eine Medienkompetenzvermittlung im Sinne eines Lernens über Medien beschränkt. Vielmehr scheint es einen Konsens zu geben, dass ein Lernen über Medien mit einem Lernen mit Medien erfolgen soll. Diese Perspektive zeigt in älteren Positionspapieren und Stellungnahmen (z. B. BMBF 2010; KBoM 2011; KMK 2012) sowie in aktuellen Publikationen (z. B. LKM 2015; BMBF 2016; KMK 2016a). Die Vision eines Lernens mit und über digitale Medien knüpft an das enge Verständnis einer integrativen Medienbildung an, wie es bereits von der Arbeitsgruppe Erziehungswissenschaft (1997) diskutiert wurde (Kap. 1.1). Dieses Kapitel zeigt, dass das Lernen mit und über (digitale) Medien eine zentrale Leitidee markiert. Zudem werden exemplarische Einblicke gegeben, was unter einem Lernen mit sowie einem Lernen über Medien in bildungspolitischen und -administrativen Kontexten verstanden wird.

Bei Betrachtung der älteren Publikationen des BMBF (2010) und der KMK (2012) erfolgt die Verknüpfung des Lernens mit und über Medien vor dem Hintergrund der sich durch Medien eröffnenden Möglichkeiten und Chancen. «[D]urch ihr vielfältiges 
didaktisch-methodisches Potenzial» können Medien, so die Argumentation der KMK $(2012,4)$ «als Motor und Motivator für das Lehren und Lernen in der Schule» betrachtet werden. In diesem Kontext wird von der KMK $(2012,4)$ exemplarisch auf Ansätze des selbstgesteuerten oder kooperativen Lernens verwiesen. Neben der Betonung weiterer Potenziale markiert die KMK (2012, 4), dass Medien «nicht nur Bildungsprozesse und Lerntransfers in methodisch-didaktischem Sinne fördern; sie werden selbst zum Bildungsgegenstand. Schulische Medienbildung umfasst also stets das Lernen mit Medien und das Lernen über Medien». Im Rahmen des Expertenberichtes des BMBF $(2010,7)$ werden vor allem digitale Medien betrachtet sowie die sich damit entwickelnden neuen «Lern- und Arbeitsformen, die sowohl für Bildungs- als auch für Arbeitsprozesse einschneidende Veränderungen mit sich bringen». Vor diesem Hintergrund umfasst Medienbildung «Medien als Gegenstand des Lernens und das in hohem Maße selbst gesteuerte und selbst verantwortete Lernen mit Medien, das im Lebensverlauf zunehmende Bedeutung gewinnt und eingeübt sein will» (BMBF 2010, 7). In den Forderungen der Initiative KBoM (2011) bezeichnet der Medienbildungsbegriff eine «umfassende und integrierende Perspektive auf medienbezogene, lebenslange Bildungsprozesse für alle Ziel- und Altersgruppen» (ebd., 6). Wenngleich dieses Verständnis nicht auf die Bildungsinstitution Schule beschränkt bleibt, wird auch in den Forderungen der KBoM (2011) auf ein Lernen mit und über Medien verwiesen. Insbesondere die Betonung eines handlungsorientierten Ansatzes, mit dem «sowohl die Probleme und Risiken in den Medien präventiv und kritisch-reflexiv angegangen als auch das positive und kreative Potenzial von Medien für formale und informelle Bildungskontexte handlungsorientiert eröffnet» (ebd.) werden, kann als ein Lernen mit und über Medien als Leitidee gedeutet werden (Kap. 4.5.1.2).

Die Verzahnung eines Lernens mit und über Medien wird auch in jüngeren Publikationen thematisiert, wenngleich Medienbildung teilweise durch die Termini «digitale Bildung» (BMBF 2016) bzw. «Kompetenzen in einer digitalen Welt» (KMK 2016a) ersetzt wurde. Mit der intendierten Betonung der Herausforderungen der Digitalisierung durch die KMK (2016a) und das BMBF (2016) geht gleichwohl eine Einschränkung des Medienbegriffs einher. Die Leitidee des Lernens mit und über Medien richtet sich vor allem auf digitale Medien. Aufgrund der Digitalisierung, so lautet beispielsweise die Argumentation der KMK (2016a, 11), welche «alle Lebensbereiche und - in unterschiedlicher Intensität - alle Altersstufen umfasst, sollte das Lernen mit und über digitale Medien und Werkzeuge bereits in den Schulen der Primarstufe beginnen». Auch im Rahmen des Strategiepapiers des BMBF (2016) wird das Lernen mit und über Medien auf digitale Medien eingeschränkt. Im Fokus «[d]igitaler Bildung» steht für das $\operatorname{BMBF}(2016,10)$ sowohl «die Vermittlung digitaler Kompetenz, d. h. der Fähigkeit zur fachkundigen und verantwortungsvollen Nutzung digitaler Medien (digitale Bildung als Lehr- und Lerninhalt), als auch das Lernen mit digitalen Medien (digitale Bildung als Instrument)». Die LKM (2015) markiert ein Lernen mit und über (digitale) 
Medien ebenfalls als Leitidee, verwendet im Gegensatz zu den vorherigen Positionen jedoch einen weiten Medienbegriff:

«Als Lernen mit und über Medien erfordert [die schulische Medienbildung] die verbindliche Integration ihrer Inhalte, Gegenstände und Ziele in die landesweiten Fachlehrpläne sowie eine umfassende Berücksichtigung bei der schulinternen Lehr- und Lernplanung.» (LKM 2015, 2)

Die exemplarischen Auszüge belegen, dass ein Lernen mit und über Medien eine konsensfähige Leitidee in bildungspolitischen und -administrativen Dokumenten markiert. Mit der Fokussierung digitaler Medien bzw. der Betonung der Digitalisierung wird in verschiedenen Publikationen (BMBF 2016; KMK 2016a) gleichwohl eine Differenz erkennbar. Die Betonung des Digitalen, welche durch die nahezu inflationäre Verwendung des Adjektivs «digital» an vielfältigen Stellen zum Ausdruck gebracht wird, beinhaltet dabei nicht nur die Implikationen für die Wahl der (Lern-)Medien, sondern auch für das Lernen über Medien. So wurde für den entwickelten Kompetenzrahmen «der Begriff «Kompetenzen in der digitalen Welt` gewählt, um den zukünftig noch stärker digital vorhandenen Zugängen zu Medien und Diensten zu entsprechen» (KMK 2016a, 14). Gleichwohl soll der entwickelte Kompetenzrahmen, so die Argumentation der KMK (2016a, 11), «mit Blick auf die konkreten Anforderungen für eine schulische «Bildung in der digitalen Welt über die bisher entwickelten Konzepte zur Medienbildung [hinausgehen]».

Ohne an dieser Stelle einen systematischen Vergleich auszuführen, wie die Leitidee des (1.) Lernens über Medien sowie (2.) des Lernens mit Medien in den verschiedenen bildungspolitischen und -administrativen Dokumenten jeweils verstanden wurde, wird hier zumindest ein exemplarischer Einblick gegeben (Tab. 4.1). Dieser Einblick kann auch einen ersten Hinweis dafür geben, inwiefern der von der KMK (2016a, 11) formulierte Anspruch in der Realisierung nachvollziehbar erscheint ${ }^{73}$. Einen ersten Überblick wie das Lernen über Medien verstanden wird, bieten die verwendeten Strukturierungsangebote zur Begründung der Relevanz von Medienbildung bzw. zur Darstellung relevanter Kompetenzen und Fähigkeiten (Tab. 4.1). Im Rahmen des früheren Beschlusses der KMK (2012, 3 ff.) wird die «Bedeutung der Medienbildung in der Schule» beispielsweise in Form von fünf verschiedenen Dimensionen erläutert. Im Gegensatz dazu werden im Bericht der Expertenkommission des BMBF (2010, 8 ff.) sowie im aktuellen Strategiepapier der KMK (2016a, 15 ff.) Kompetenzen bzw. Performanzen formuliert, welche jeweils übergeordneten Themenfeldern bzw.

73 Bedingt durch die unterschiedlichen Publikationstypen und Veröffentlichungskontexte fällt die Präzisierung der zentralen Leitideen teilweise sehr unterschiedlich aus. Die Ausführungen reichen von mehrdimensionalen Kompetenzmodellen (LKM 2015; KMK 2016a), Konkretisierungen spezifischer Themen- und Aufgabenfelder (BMBF 2010) über die Beschreibung vielfältiger Bedeutungsebenen (KMK 2012) bis hin zu prägnanten Formulierungen, die sich auf wenige Sätze bzw. Absätze begrenzen (BMBF 2016). 
Kompetenzbereichen zugeordnet werden. Formal zeigt sich dabei zunächst, dass im Strategiepapier der KMK (2016a, 15 ff.) zwischen mehr Kompetenzbereichen unterschieden wird als in den vorherigen Veröffentlichungen. In Betrachtung der inhaltlichen Bedeutung und Reichweite der verschiedenen Bereiche fällt auf, dass der Aspekt der «Identitätssuche und Orientierung» (BMBF 2010, 9) bzw. «Identitätsbildung und Persönlichkeitsentwicklung» KMK $(2012,5)$ in den sechs Kompetenzbereichen nicht explizit thematisiert wird. Hinsichtlich der Abstraktionsgrades kann markiert werden, dass die Bezeichnung der Kompetenzbereiche des Strategiepapiers der KMK (2016a, 15 ff.) sich jeweils aus einer Kombination von zwei Aktivitäten bzw. Performanzen zusammensetzt. Die Bedeutungsdimensionen im Beschluss «Medienbildung in der Schule» (KMK 2012, 3 ff.) sind dagegen offener formuliert.

\begin{tabular}{|c|c|c|}
\hline $\begin{array}{l}\text { Themen- und Aufgabenfelder } \\
\text { des BMBF (2010) }\end{array}$ & $\begin{array}{l}\text { Bedeutungsdimensionen der } \\
\text { KMK (2012) }\end{array}$ & $\begin{array}{l}\text { Kompetenzbereiche der KMK } \\
\text { (2016a) }\end{array}$ \\
\hline Information und Wissen & $\begin{array}{l}\text { Unterstützung und Gestaltung } \\
\text { innovativer und nachhaltiger } \\
\text { Lehr- und Lernprozesse }\end{array}$ & $\begin{array}{l}\text { Suchen, Verarbeiten und Auf- } \\
\text { bewahren }\end{array}$ \\
\hline $\begin{array}{l}\text { Kommunikation und Kooper- } \\
\text { ration }\end{array}$ & $\begin{array}{l}\text { Selbst bestimmte, aktive und } \\
\text { demokratische Teilhabe an } \\
\text { Politik, Kultur und Gesell- } \\
\text { schaft }\end{array}$ & $\begin{array}{l}\text { Kommunizieren und Koope- } \\
\text { rieren }\end{array}$ \\
\hline $\begin{array}{l}\text { Identitätssuche und Orien- } \\
\text { tierung }\end{array}$ & $\begin{array}{l}\text { Identitätsbildung und Persön- } \\
\text { lichkeitsentwicklung }\end{array}$ & Produzieren und Präsentieren \\
\hline \multirow[t]{2}{*}{$\begin{array}{l}\text { Digitale Wirklichkeiten und } \\
\text { produktives Handeln }\end{array}$} & $\begin{array}{l}\text { Ausprägung moralischer Hal- } \\
\text { tungen, ethischer Werte und } \\
\text { ästhetischer Urteile }\end{array}$ & Schützen und sicher Agieren \\
\hline & $\begin{array}{l}\text { Schutz von Kindern und } \\
\text { Jugendlichen vor negativen } \\
\text { Einflüssen und Wirkungen von } \\
\text { Medien }\end{array}$ & Problemlösen und Handeln \\
\hline
\end{tabular}

Tab. 4.1: Schematische Gegenüberstellung der formulierten Zieldimensionen der Publikationen der KMK (2012, 2016a) sowie des BMBF (2010).

In der exemplarischen Betrachtung der Teilbereiche «Kommunikation und Kooperation» (BMBF 2010, 9), «[s]elbst bestimmte, aktive und demokratische Teilhabe an Politik, Kultur und Gesellschaft» (KMK 2012, 4) sowie "Kommunizieren und Kooperieren» (KMK 2016a, 16) lassen sich weitere Unterschiede hinsichtlich der Abstraktionsebene relevanter Fähigkeiten und Kompetenzen zum Lernen über Medien formulieren. Im Rahmen des früheren Beschlusses der KMK $(2012,4)$ erfolgt die Darstellung der «Bedeutung der Medienbildung in der Schule» u. a. durch die folgende Formulierung: 
«Die Kommunikations- und Massenmedien sind konstitutiver Bestandteil der gesellschaftlichen Wirklichkeit; sie gestalten politische Prozesse mit, beeinflussen die öffentliche Meinungsbildung und entscheiden in hohem Maße über Umfang und Art gesellschaftlicher Teilhabe. Ob neue Medien dabei zu mehr Demokratie und zu mehr gesellschaftlicher Freiheit führen oder aber das eine wie das andere auch einschränken und bedrohen können, hängt wesentlich von ihrem kompetenten Gebrauch ab. Mangelnde Medienkompetenz beschränkt die Möglichkeiten des Einzelnen zur politischen Mitwirkung und kulturellen Partizipation» (KMK 2012, 4).

In dieser Formulierung kann Medienkompetenz als Erweiterung der Möglichkeiten zur «politischen Mitwirkung und kulturellen Partizipation» verstanden werden. Medienbildung wird in diesem Kontext von der KMK $(2012,5)$ als Aspekt «politischer Bildung» verstanden. Medienbildung, so die Formulierung der KMK $(2012,5)$ «trägt damit zur Ausgestaltung unserer auf Freiheit, Gerechtigkeit und Solidarität ausgerichteten Gesellschaft bei» (KMK 2012, 5). Mit dem Bezug zu diesen übergeordneten Normen bleiben die Ausführungen in diesem Teilbereich eher abstrakt und stellen in der Praxis handelnde Lehrerinnen und Lehrer vor das «Problem der Normen-Ableitung» (Jank und Meyer 2008, 123 ff.). Im Gegensatz dazu sind die formulierten Performanzen des BMBF $(2010,9)$ sowie der KMK $(2016 a, 16)$ konkreter gefasst. Neben vier weiteren Bereichen formuliert das $\operatorname{BMBF}(2010,9)$ beispielsweise die zwei folgenden Ziele: «mit- und voneinander lernen in und mit [d] igitalen Medien» sowie «sich bei der Herstellung von Produkten und Dienstleistungen auf (virtuelle) Gemeinschaften stützen und sich an deren Entstehung, Erhalt und Verbreitung beteiligen». Im Strategiepapier der KMK (2016a, 16) wird der Kompetenzbereich «Kommunizieren und Kooperieren» zunächst in fünf Teilbereiche untergliedert, welche jeweils zwei bis vier Performanzen beinhalten. Exemplarische Formulierung sind «Digitale Werkzeuge für die Zusammenarbeit bei der Zusammenführung von Informationen, Daten und Ressourcen nutzen» sowie "Als selbstbestimmter Bürger aktiv an der Gesellschaft teilhaben». In Betrachtung dieser Performanzbeschreibungen lässt sich festhalten, dass der Abstraktionsgrad an zu erwerbenden Fähigkeiten und Kompetenzen zum Lernen über Medien im Strategiepapier KMK (2016a, 16) präziser erfolgt als im früheren Beschluss der KMK (2012). Zugleich wird sichtbar, dass sich auch die Abstraktionsebenen der einzelnen Performanzen zum Teil deutlich unterscheiden. So bleibt im Teilbereich «An der Gesellschaft aktiv teilhaben» und der exemplarischen Performanz «Als selbstbestimmter Bürger aktiv an der Gesellschaft teilhaben» auch im aktuellen Strategiepapier der KMK $(2016 a, 16)$ relativ offen, wie dies über ein Lernen über Medien möglich wird. 
Die Leitidee des Lernens mit Medien wird in den Publikationen unterschiedlich zum Ausdruck gebracht. Im Expertenbericht des BMBF $(2010,7)$ wird das «Lernen mit Medien» im Kontext der sich durch digitale Medien neu entwickelnden «Lernund Arbeitsformen» begründet. So werden für «Bildungs- und Arbeitsprozesse entscheidende Veränderungen» vom $\operatorname{BMBF}(2010,7)$ erwartet. Entsprechend müsse Medienbildung auch das Lernen mit Medien umfassen. Im Beschluss der KMK (2012, 4) wird das Lernen mit Medien als eigene Bedeutungsdimension dargestellt. Die Begründung zum Lernen mit Medien erfolgt mit Bezug auf ihr «vielfältiges didaktischmethodisches Potenzial» (KMK 2012, 4). So werden verschiedene Möglichkeiten markiert, die einen sachgerechten Einsatz von Medien im Unterricht ermöglichen. Am umfangreichsten wird das Lernen mit Medien im aktuellen Strategiepapier der KMK (2016a) markiert. Neben der Integration relevanter Kompetenzen in die Lehr- und Bildungspläne der Länder, wird der systematische Einsatz digitaler Lernumgebungen gefordert (KMK 2016a, 11 f.). Die Begründung dieser Zielstellung erfolgt im Strategiepapier der KMK (2016a) sowohl über die Betonung neuer Möglichkeiten, als auch über Erwartungen zu Veränderungen von zukünftig relevantem Lernen. So werden "die Individualisierungsmöglichkeit und die Übernahme von Eigenverantwortung bei den Lernprozessen gestärkt» (KMK 2016a, 12), sofern ein sinnvoller Einsatz digitaler Medien erfolgt. Zudem «rückt [beim Lernen] weniger das reproduktive als das prozess- und ergebnisorientierte - kreative und kritische - Lernen in den Fokus» (KMK 2016a, 13). Ausserdem wird der Einsatz digitaler Medien mithilfe klassischer E-Learning-Argumente begründete, wie beispielsweise die Orts- und Zeitunabhängigkeit des Lernens (KMK 2016a, 13).

\subsubsection{Integrativer Ansatz statt eigener Lernbereich}

Die Umsetzung des Bildungsauftrages einer Medienbildung soll als integraler Bestandteil aller Unterrichtsfächer erfolgen. Diese Perspektive markiert, neben der Auffassung von Medienbildung als Erziehungs- und Bildungsauftrag sowie der Leitidee eines Lernens mit und über Medien, einen dritten Konsens in bildungspolitischen und -administrativen Dokumenten zur Medienbildung bzw. digitaler Bildung. Statt einen eigenen Lernbereich oder ein spezifisches Fach «Medienkunde» zu etablieren, wird der Ansatz einer integrativen Medienbildung bevorzugt, wie er bereits von der Arbeitsgruppe Erziehungswissenschaft (1997) diskutiert wurde (Kap. 1.1). Dieser Konsens wird zum einen durch die Formulierung von Zukunftsaufgaben und Forderungen zum Ausdruck gebracht (z. B. BMBF 2010; KMK 2012, 2016a). Zum anderen finden sich Problematisierungen der aktuellen Situation zur Hervorhebung der aktuellen Handlungsrelevanz (z. B. BMBF 2010; KBoM 2011; Bundestag 2015). Im Zusammenhang mit dieser Umsetzungsstrategie stehen verschiedene Implikationen zur Veränderung von Lehr- und Bildungsplänen sowie erweiterte Aufgaben von Lehrerinnen und Lehrer. 
Bereits im Expertenbericht des BMBF $(2010,13)$ wurde darauf aufmerksam gemacht, dass die Medienbildung in der Schule als integrative Aufgabe betrachtet wird bzw. «in der Schule integrativ in unterschiedlichen Fächern stattfinden soll». Zugleich markiert das BMBF $(2010,13)$ die Problematik, dass «es nur wenige Ansätze [gibt], Medienbildung als kontinuierlichen Prozess über verschiedene Fächer und Jahrgangsstufen hinweg in systematischer und koordinierter Weise zu realisieren». Zur verpflichtenden und flächendeckenden Verankerung müssen Lösungen entwickelt werden, so lautet die Forderung des Expertenberichts des (BMBF 2010, 13), welche vor allem an die Bildungspolitik gerichtet sind. In ähnlicher Weise problematisiert die Initiative KBoM $(2011$, 8), dass sich die «Förderung von Medienkompetenz und Medienbildung als Querschnittsaufgabe für alle Fächer bislang nicht durchgesetzt [hat]. Zwar sind in den Bildungs- und Lehrplänen der Bundesländer medienpädagogische Themen in unterschiedlicher Differenzierung enthalten, aber die Verbindlichkeit fehlt» (ebd.). Zudem problematisiert die KBoM $(2011,8)$ die fehlende "grundlegende medienpädagogische Qualifizierung aller Lehrkräfte». Zum Umgang mit diesem problematisierten Ist-Zustand forderte die $\operatorname{KBoM}(2011,5)$ u. a. eine verbindliche Verankerung medienpädagogischer Themen «in den Curricula und Prüfungen aller Schularten» sowie «die Verankerung einer medienpädagogischen Grundbildung als verbindlicher und prüfungsrelevanter Bestandteil in allen pädagogischen Studiengängen und Ausbildungsbereichen ${ }^{74}$. Die Argumentationsfigur einer Problematisierung des Ist-Zustandes wurde in jüngerer Vergangenheit auch für die Formulierung eines Koalitionsantrages im deutschen Bundestag (2015) verwendet. So problematisiert der Koalitionsantrag die häufig ausbleibende Integration digitaler Medien in die Unterrichtsgestaltung. Zudem wird das «Wissen über und [der] Umgang mit digitalen Medien» von deutschen Schülerinnen und -Schülern mit Verweis auf die ICILS ${ }^{75}$ (Bos et al. 2014) als kritisch eingeschätzt: «Eine solche Momentaufnahme steht im Widerspruch zum Anspruch Deutschlands, eine fortschrittliche Bildungsnation zu sein» (Bundestag 2015, 2). Vor diesem Hintergrund wird u. a. eine Verankerung «der Vermittlung von digitaler Medienkompetenz sowie digitalen Bildungsinhalten fächerübergreifend in die Bildungspläne und in die länderspezifischen Qualitätsrahmen zur Schulentwicklung [gefordert]» (Bundestag 2015, 5).

Die Betonung eines integralen bzw. integrativen Konzeptes erfolgt insbesondere in den Veröffentlichungen der KMK $(2012,2016 a)$. So wird im Beschluss «Medienbildung in der Schule» (KMK 2012) darauf hingewiesen, dass "Medienbildung in der Schule [...] kein Unterrichtsfach» ist und es deshalb umso wichtiger sei, «die

$74 \quad$ Neben den Forderungen auf dem Kongress wurde die Forderungen nach einer medienpädagogischen Grundbildung in einem weiteren Papier gefordert (KBoM 2014).

75 ICILS steht für «International Computer and Information Literacy Study» (Fraillon et al. 2014). Die Durchführung dieser Studie in Deutschland trägt den Untertitel: «Computer- und informationsbezogene Kompetenzen von Schülerinnen und Schülern in der 8. Jahrgangsstufe im internationalen Vergleich (Bos et al. 2014). 
Voraussetzungen für eine umfassende, alle Fächer einbeziehende Medienbildung in der Schule zu schaffen» (KMK 2012, 6). Zugleich bleibt der Verpflichtungscharakter dieses Beschlusses lediglich auf der Ebene einer Empfehlung. Hinsichtlich der Überarbeitung von Lehr- und Bildungsplänen wird beispielsweise die «Aktualisierung und Akzentuierung der Medienbildung in den einzelnen Fächern und die Formulierung eigener fächerübergreifender Kriterien zur Medienbildung» von der KMK $(2012,6)$ als «wünschenswert» bezeichnet. Deutlich verbindlicher kann das Strategiepapier der KMK $(2016 a, 6)$ verstanden werden. Wie bereits skizziert, verpflichten sich die Länder zur Vermittlung der «Kompetenzen in einer digitalen Welt» für «alle Schülerinnen und Schüler, die zum Schuljahr 2018/2019 in die Grundschule eingeschult werden oder in die Sek I eintreten» KMK (2016a, 6). Die Realisierung soll als integrativer Bestandteil der Fächer erfolgen:

«Dies wird nicht über ein eigenes Curriculum für ein eigenes Fach umgesetzt, sondern wird integrativer Teil der Fachcurricula aller Fächer. Jedes Fach beinhaltet spezifische Zugänge zu den Kompetenzen in der digitalen Welt durch seine Sach- und Handlungszugänge. Damit werden spezifische Fach-Kompetenzen erworben, aber auch grundlegende (fach-)spezifische Ausprägungen der Kompetenzen für die digitale Welt. Die Entwicklung der Kompetenzen findet auf diese Weise (analog zum Lesen und Schreiben) in vielfältigen Erfahrungs- und Lernmöglichkeiten statt» (KMK 2016a, 11 f.).

Zur Realisierung dieser Umsetzungsidee lassen sich vielfältige Herausforderungen markieren, von denen für diese Arbeit zwei Aspekte besonders erwähnenswert sind: (1.) die Erweiterung des Aufgabenspektrums von Lehrerinnen und Lehrer sowie (2.) die notwendige Qualifikation zur Bewältigung dieser neuen Aufgaben. Im Strategiepapier der KMK (2016a, 28) wird beispielsweise explizit darauf hingewiesen, dass sich die Anforderungen, welche «die Bildungs- und Erziehungsprozesse in der digitalen Welt m mit sich bringen», so die KMK (2016a, 28) weiter, "das Aufgabenspektrum aller Lehrkräfte dauerhaft quantitativ und qualitativ erheblich [erweitern]». Die Vermittlung von Kompetenzen in einer digital geprägten Kultur kann in diesem Sinne als ein neues Aufgabenfeld markiert werden. Um diese Aufgabe angemessen bearbeiten zu können, ist eine Bedingung, so die Argumentation der KMK (2016a, 24), dass alle Lehrkräfte «selbst über allgemeine Medienkompetenz verfügen und in ihren fachlichen Zuständigkeiten zugleich ‘Medienexperten` werden». Die bildungspolitische Relevanz einer angemessenen Lehrerinnen- und Lehrerbildung als Voraussetzung für eine angemessene Medienbildung in der Schule wird auch in verschiedenen weiteren Dokumenten bestätigt (z. B. KMK 2012; BMBF 2016; KBoM 2014, 2011; Bundestag 2015). 


\subsubsection{Implikationen und Herausforderungen für die Lehrerinnen- und Lehrerbildung}

Bei Betrachtung der drei skizzierten Leitideen markiert der Begriff Medienbildung - verstanden als «breitbandiges Konzept» zur pädagogisch strukturierten Medienkompetenzvermittlung (Jörissen 2011, 214) - ein zentrales Leitbild für die Schule. Neben der Vermittlung von Medienkompetenz umfasst dieses Leitbild auch ein Lernen mit Medien (Kap. 4.2.1.2) sowie die Konzeptualisierung von Medienbildung als integrativen Ansatz aller Schulfächer (Kap. 4.2.1.3). Im Zusammenhang mit diesen Leitideen stehen vielfältige Herausforderungen für Lehrkräfte sowie für die Lehrerinnen- und Lehrerbildung. Um den komplexen Anforderungen zur Medienbildung in der Schule gerecht werden zu können, bedarf es einer angemessenen Vorbereitung und Begleitung von Lehrkräften in allen Phasen der Lehrerinnen- und Lehrerbildung. Umso überraschender ist es, dass es im Gegensatz zu den vielfältigen Leitbildern für die schulische Medienbildung kaum vergleichbare Leitbilder für Lehrerinnen- und Lehrerbildung identifiziert werden konnten. Zudem sind die existierenden bildungspolitischen und -administrativen Dokumente zur Medienbildung in der Lehrerinnenund Lehrerbildung sowie die aktuelle Situation der Praxis kritisch zu bewerten. Diese Kritik begründet sich sowohl vor dem Hintergrund der skizzierten Leitbilder zur schulischen Medienbildung sowie bei Betrachtung des medienpädagogischen Fachdiskurses.

Zur Beschreibung der Medienbildung in der Lehrerinnen- und Lehrerbildung in Deutschland erfolgt in Anlehnung an Kammerl und Mayrberger (2011, 175 f.) ein Blick in aktuelle Rahmenpläne und Studienordnungen. Kammerl und Mayrberger (2011, 176) diskutieren dafür die bildungswissenschaftlichen Standards für der Lehrerinnen- und Lehrerbildung der Kultusministerkonferenz (KMK 2004). In Auseinandersetzung mit den überschaubaren Bezügen zu medienbezogenen Fähigkeiten und Kenntnissen im Rahmen der Standards kommen Kammerl und Mayrberger $(2011,176)$ zu einem aus medienpädagogischer Perspektive ernüchternden Urteil. Für Kammerl und Mayrberger $(2011,176)$ wird deutlich, dass der Beschluss zu den Standards «von verbindlichen Vorgaben zur Förderung einer grundlegenden Medienbildung von Lehrerinnen und Lehrern über die gesamte Dauer ihrer Berufstätigkeit recht weit entfernt ist». So lassen sich zwar einerseits «eher mediendidaktisch ausgerichtete[...] Standards» identifizieren. Anderseits fehlen Kammerl und Mayrberger $(2011,176)$ zentrale Aspekte, wie z. B. «die Förderung von umfassender Medienkompetenz durch eine integrierte Medienerziehung und gezielte Förderung von Prozessen der Medienbildung». Aus medienpädagogischer Perspektive müssen die Standards für die Lehrerinnen- und Lehrerbildung im Bereich der Bildungswissenschaften der KMK (2004) als problematisch bezeichnet werden.

Die von Kammerl und Mayrberger (2011) formulierte Kritik an den Standards im Bereich der Lehrerinnen- und Lehrerbildung trifft auch für die von der KMK (2008) formulierten inhaltlichen Anforderungen der «Fachwissenschaften und Fachdidaktiken 
in der Lehrerbildung» zu. Zum einen sind die expliziten Bezüge zu Medien oder medienbezogenen Kompetenzen in der Gesamtbetrachtung des Dokumentes überschaubar. Bei den im Studium aufzubauenden Kompetenzen finden sich Bezüge zu Medien in dem Bereich «[ü]ber Erkenntnis- und Arbeitsmethoden der Fächer verfügen» (KMK 2008, 4). Dabei sollen Studierende die fachspezifischen «Erkenntnis- und Arbeitsmethoden und Medien» kennen und in der Lage sein, diese «adressaten- und sachgerecht anzuwenden». Die Kompetenz, diese Fähigkeiten und Kenntnisse zu vermitteln wird nicht thematisiert. In den zwei weiteren Bereichen «Fachwissen» und «fachdidaktisches Wissen» finden sich keine Bezüge zu Medien. Im Bereich des Vorbereitungsdienstes sowie der Fort- und Weiterbildung finden sich ebenfalls keine Bezüge zu Medien. In Betrachtung der verschiedenen Fachprofile lassen sich analog zu den Standards der Bildungswissenschaften eher mediendidaktische Kompetenzen identifizieren. So werden Medien vor allem als «Unterrichtsmedien» ${ }^{76}$ bezeichnet, es geht also um den Einsatz respektive die Nutzung fachangemessener Medien ${ }^{77}$. Für den Bereich der Biologie beschränken sich die Medienbezüge beispielsweise auf die Kompetenzformulierung «können Unterrichtskonzepte und -medien fachgerecht gestalten, inhaltlich bewerten, neuere chemische/biologische Forschung in Übersichtsdarstellungen verfolgen, um sie in den Unterricht einzubringen» (KMK 2008, 20).

Mit dem Beschluss «Medienbildung in der Schule» im Jahr 2012 werden die von Kammerl und Mayrberger $(2011,176)$ markierten Probleme von der KMK (2012) berücksichtigt und thematisiert. So wird «Medienbildung» von der KMK $(2012,9)$ zum «Bildungsauftrag der Schule» erklärt. Zur Realisierung dieses Bildungsauftrages werden ferner verschiedene Handlungsfelder markiert, um «die Voraussetzungen für eine umfassende, alle Fächer einbeziehende Medienbildung in der Schule zu schaffen» (KMK 2012, 6). Die ersten zwei Handlungsfelder der KMK $(2012,6)$ umfassen die «Lehr- und Bildungspläne» der Schulen sowie die «Lehrerbildung». So heisst es in diesem Beschluss explizit: «In diesem Sinne ist Medienbildung sowohl in den Bildungswissenschaften als auch in der fachbezogenen Lehrerausbildung der ersten und zweiten Phase in den Prüfungsordnungen ausreichend und verbindlich zu verankern» (KMK 2012, 7). Als Konsequenz dieses Beschlusses liegt die Erwartung nahe, dass folgende Veränderungen der Standards für die Lehrerinnen- und Lehrerbildung den Versuch unternehmen, dem Bildungsauftrag der Medienbildung gerecht zu werden. Die im Jahr 2014 veränderte Fassung der «Standards für die Lehrerbildung: Bildungswissenschaften (KMK 2014) sowie die im Jahr 2016 veränderte Fassung der «Ländergemeinsame[n] inhaltliche[n] Anforderungen für die Fachwissenschaften und Fachdidaktiken in der Lehrerbildung» (KMK 2016b) umfassen jedoch keine Änderung hinsichtlich der von Kammerl und Mayrberger (2011) kritisierten Aspekte. Vor

76 Belege für eine entsprechende Formulierung bieten u. a. die Kompetenzprofile der Fächer Biologie (KMK 2008, 20), Chemie (ebd., 22), Geschichte (ebd., 29) Informatik (ebd., 32), Physik (ebd., 45).

77 Belege für eine entsprechende Formulierung bieten u. a. die fachdidaktischen Studieninhalte des Fachs Arbeit, Wirtschaft, Technik (KMK 2008, 11) sowie der Geographie (ebd., 21). 
dem Hintergrund der vielfältigen Modelle und Konstrukte zur zeitgemässen Konzeptualisierung medienpädagogischer Kompetenz von Lehrkräften (z. B. Blömeke 2005; Moser 2010b; Mayrberger 2012a; Tulodziecki 2012) sind die Standards weiterhin als problematisch zu bewerten.

\subsubsection{Praxis der Medienbildung im Lehramtsstudium der TU Darmstadt}

Der Aufbau der folgenden Ausführungen zur Praxis der Medienbildung im Lehramtsstudium der TU Darmstadt gliedert sich in zwei Teile. Im ersten Schritt wird in Betrachtung der Studienordnung der Grundwissenschaften skizziert, welche Möglichkeiten sich zum Lernen mit digitalen Medien eröffnen und welche Grenzen dabei existieren. Im Rahmen der ersten Phase des QSL-Projektes ${ }^{78}$ "Digitale Lehrerbildung» wurde dabei sichtbar, dass die Fächerauswahl der Studierenden auch die Möglichkeiten zum Lernen mit digitalen Medien vorbestimmt. Der Einsatz digitaler Medien ist nicht allen Fachdisziplinen der Lehrerinnen- und Lehrerbildung verankert (Kap. 4.2.2.1). Im zweiten Schritt wird auf struktureller Ebene aufgezeigt, dass ein Lernen über Medien nur ein optionaler Bereich im Lehramtsstudium der Grundwissenschaft darstellt. Entsprechend ist ein Lernen über Medien nur denjenigen Studierenden möglich, welche diese Wahloption angenommen haben (Kap. 4.2.2.2).

\subsubsection{Begrenzte Möglichkeiten zum Lernen mit digitalen Medien}

Die Formulierung des Lernens mit Medien umfasst in den folgenden Ausführungen zwei Bedeutungsebenen. Auf der ersten Ebene umfasst das «Lernen mit Medien» im wörtlichen Sinne Situationen, in denen Lehramtsstudierende die Gelegenheit erhalten, mit digitalen Medien etwas zu lernen. Im Fokus dieser Ebene steht vor allem die Praxis der Hochschullehre bzw. des Studiums und welche Erfahrungen Studierenden dabei ermöglicht werden. Auf der zweiten Ebene umfasst das «Lernen mit Medien» didaktisches und lerntheoretisches Theoriewissen, um Lehr- und Lernsituationen mit digitalen Medien analytisch bewerten und professionell gestalten zu können. Im Fokus dieser Ebene steht die Absicht, Lehramtsstudierende zu befähigen, als angehende Expertinnen und Experten für das Lehren und Lernen eine mediendidaktische Perspektive, einnehmen zu können. Beiden Ebenen ist gemeinsam, dass Medien als Mittel der Vermittlung verstanden bzw. als Lerninstrumente konzeptualisiert werden. Das Ziel der folgenden Ausführungen ist die Beantwortung der Frage, inwiefern Studierenden im Lehramtsstudium Möglichkeiten zum Lernen mit Medien (auf beiden Bedeutungsebenen) eröffnet werden.

78 QSL-Mittel dienen der Verbesserung der Qualität der Studienbedingungen und der Lehre an der TU Darmstadt. Weitere Informationen sind online verfügbar unter https://www.intern.tu-darmstadt.de/ dez_ii/ studienprogramme/qsl_mittelvergabe_2/qsl_mittelvergabe.de.jsp (01.08.2018). 
Diese Frage kann strukturell auf Basis der Studienordnungen beantwortet werden. Das Lernen mit Medien kann in Anlehnung an Schiefner-Rohs (2013a) als bildungstechnologisch-optimierende Perspektive vereinfacht werden: Es gilt zu klären, wie neue Medien zur Optimierung von Lehr- und Lernarrangements verwendet werden können. Auch für Ruge $(2014,188)$ steht bei dieser Betrachtungsweise von Medien die Frage im Fokus, «wie sich (formelle) Bildungsprozesse, wie z. B. Schulunterricht oder die Hochschuldidaktik, durch den Einsatz von Medien optimieren lassen und ob durch neue Technologien auch neue Möglichkeiten entstehen, die es zu nutzen gilt» (ebd.). Ruge $(2014,188)$ bezeichnet diese Perspektive «klassischerweise als Mediendidaktik». Die Frage nach dem didaktischen Mehrwert ist häufig mit dieser Perspektive verbunden. Medien sind in diesem Verständnis als Mittel oder Werkzeug der Vermittlung zu verstehen. Für (künftige) Lehrkräfte gilt es zu lernen, wie diese didaktisch angemessen eingesetzt werden können.

Bei Betrachtung der Studienordnung der Grundwissenschaften werden neben den zentralen Studienzielen auch «zentrale Kompetenzen in der grundwissenschaftlichen Ausbildung» (TU Darmstadt 2009, 3) markiert. Im Rahmen der aufgelisteten zehn Kompetenzen findet sich die Formulierung: «Den Einsatz neuer Medien pädagogisch begründen und argumentativ vertreten» (ebd.). Wenngleich die Formulierung des «Einsatzes» von Medien Anknüpfungspunkte zum Lernen mit Medien eröffnet, können pädagogische Begründungen zur Verwendung neuer Medien auch über ein Mittelverständnis von Medien hinausgehen. Bei dieser Formulierung bleibt insofern zunächst offen, inwiefern der pädagogische Einsatz neuer Medien ein Lernen mit Medien, ein Lernen über Medien oder im Sinne von Petko (2011) eine Sensibilisierung für Medien mithilfe von Medien fokussiert. Stattdessen lässt sich die Aussage treffen, dass die Studienordnung - metaphorisch formuliert - mit Bezug auf die zentralen Zielkompetenzen zunächst einen weiten Möglichkeitsraum eröffnet.

Daran anknüpfend lässt sich feststellen, dass die identische bzw. ähnliche Formulierung auch zur Konkretisierung der Zielebene einzelner Module verwendet wird. «Den Einsatz neuer Medien pädagogisch begründen und argumentativ vertreten [zu können]» (TU Darmstadt 2009, 3), wird als Zielstellung im Pflichtmodul «P2: Didaktik, Methodik und Medien» (TU Darmstadt 2009, 3) verwendet. Für das Wahlpflichtmodul «WP1: Informationspädagogik» (TU Darmstadt 2009, 36) findet sich die Formulierung: «für die pädagogische Sinnhaftigkeit selbst entwickelter Formen des Medieneinsatzes argumentativ (auch in Bezug auf kritische Nachfrage) eintreten». Unter Berücksichtigung der angegebenen Inhalte in den jeweiligen Modulbeschreibungen lässt sich für die jeweiligen Module präziser bestimmen, inwiefern diese strukturellen Möglichkeiten zum Lernen mit Medien eröffnen sollten.

Das Thema bzw. der Inhalt des Pflichtmoduls «Didaktik, Methodik und Medien» (TU Darmstadt 2009,3) ist gemäss des Modulhandbuchs die «Analyse und Reflexion der Bedingungen, Ziele, Inhalte, Formen und Mittel von Unterricht» (TU Darmstadt 
2009, 3). Mit dieser - an die Strukturmerkmale des Berliner Modells (Heinmann 1962) erinnernden - Präzisierung des Modulthemas, scheint die Lesart möglich, Medien sowohl als Mittel als auch als Inhalt des Unterrichts zu verstehen. In der Konsequenz daran eröffnet die Modulbeschreibung für Lehrende die Option, Medien als Mittel des Unterrichts zu diskutieren. Inwiefern Studierenden in der Praxis Möglichkeiten zum Lernen mit Medien eröffnet werden, kann gleichwohl nicht explizit beantwortet werden. Vielmehr lässt sich sagen, dass im Rahmen des Pflichtmoduls die Lehrenden der drei Modulveranstaltungen auf Basis der Studienordnung die Legitimation - oder zugespitzter formuliert die Pflicht - haben, Möglichkeiten zum Lernen mit neuen Medien zu eröffnen. Die drei Modulveranstaltungen umfassen gemäss der Studienordnung das Seminar oder die Vorlesung «Allgemeine Didaktik», die Vorlesung «Pädagogik der Neuen Medien» sowie das Seminar «Schulentwicklung». Von diesen drei Veranstaltungen sind von den Studierenden zwei Veranstaltungen zu besuchen. Hinsichtlich der formalen Bezeichnung der Veranstaltungen scheint es naheliegend, dass sowohl in der Veranstaltung zur Allgemeinen Didaktik als auch in der Vorlesung zur «Pädagogik der Neuen Medien» Möglichkeiten zum Lernen mit Medien bzw. Einblicke in eine didaktische Perspektive auf Medien eröffnet werden. Entsprechend hätten Studierende gemäss der Studienordnung verschiedene Möglichkeiten zum Lernen über Medien im Rahmen des Pflichtbereichs der Grundwissenschaften.

Das Thema bzw. der Inhalt des Wahlpflichtmoduls «Informationspädagogik» (TU Darmstadt 2009, 6) ist gemäss des Modulhandbuchs sehr knapp formuliert: «Einsatz Neuer Medien in Bildungsprozessen» (ebd., 3). In dieser Formulierung wird zum einen erneut der Begriff des Einsatzes verwendet. Offen bleibt hier die Frage, von wem neue Medien eingesetzt werden. Zum anderen eröffnet eine Interpretation dieser Formulierung vielfältige Möglichkeiten zur Diskussion der Frage, welches Bildungsverständnis hier zum Ausdruck gebracht werden soll. Zur Klärung der Frage, inwiefern im Rahmen dieses Moduls strukturell Möglichkeiten zum Lernen mit Medien eröffnet werden sollten, ist es lohnenswert die weiteren Kompetenzziele zu betrachten. Verschiedene Ziele sind dabei so fokussiert formuliert, dass Medien vor allem als Mittel zu verstehen sind. Exemplarisch belegen lässt sich diese didaktisch geprägte Perspektive auf Medien mit den vier folgenden Kompetenzzielen:

- «Einsatzbereiche für Neue Medien in Bildungsprozessen hinsichtlich ihrer unterschiedlichen lernfördernden Potenziale differenzieren und begrenzen» (TU Darmstadt 2009, 6).

- «[D]ie Modellierungsfunktion der Neuen Medien beurteilen und an Beispielen aus den Studienfächern demonstrieren oder illustrieren» (ebd.).

- «Neue Medien in Bildungsprozessen so einsetzen, dass neue Möglichkeiten der Veranschaulichung und der Verständnisförderung erschlossen werden» (ebd.).

- «[D]en Einsatz Neuer Medien in Bildungsprozessen so organisieren, dass die Lernenden zu erhöhter Selbsttätigkeit und eigenverantwortlichem Lernen angeregt werden» (ebd.). 
Ähnlich wie beim vorherigen Pflichtmodul wird ein Lernen mit Medien bzw. das Einnehmen einer didaktischen Perspektive im Rahmen der Studienordnung gefordert. Daraus ergibt sich die Konsequenz, dass Studierende auf Basis der Studienordnung potenziell auch im Wahlpflichtbereich Möglichkeiten zum Lernen mit Medien haben. Daran ist jedoch die Bedingung geknüpft, dass Studierende sich für diesen Wahlpflichtbereich entscheiden.

Neben der Analyse der Studienordnung kann auf die Erkenntnisse des Forschungs- und Entwicklungsprojektes «Digitale Lehrerbildung» zurückgegriffen werden (Grell et al. 2015). In diesem Projekt wurden u. a. Veranstaltungskonzepte aufgearbeitet, in denen ein innovativer und didaktisch begründeter Einsatz von digitalen Medien für Lehramtsstudierende erfahrbar wird. Bei der Recherche von mediendidaktisch und medienpädagogisch geeigneten Lehrveranstaltungen an der TU Darmstadt zeigten sich u. a. zwei zentrale Ergebnisse:

1. «Mediendidaktisch geeignete Lehrveranstaltungen, i.S.v. Lehrveranstaltungen, in denen ein innovativer und didaktisch begründeter Einsatz von digitalen bzw. neuen Medien für angehende Lehrerinnen und Lehrer erfahrbar (und mitunter aus didaktischer Perspektive zum Thema gemacht) wird, sind in einem begrenzten Umfang vorhanden. Angeboten werden diese Veranstaltungen von interessierten und engagierte Einzelpersonen (z. B. Erik Kremser in der Physik) oder von Arbeitsgruppen mit entsprechenden Arbeits- und Forschungsschwerpunkten (z. B. AG «Mathematikdidaktik» in der Mathematik).

2. Mediendidaktisch geeignete Lehrveranstaltungen stehen insofern nur einer begrenzten Auswahl von Studierenden zur Verfügung, so dass die Fächerauswahl der Studierenden derzeit implizit auch die Möglichkeiten vorbestimmt, Lehrveranstaltungen mit einem angemessenen Einsatz digitaler Medien in den Fachdisziplinen zu erleben» (Grell et al. 2015, 7 f.).

\subsubsection{Lernen über Medien nur als Wahloption}

Die Formulierung des Lernens über Medien geht über das instrumentelle Verständnis von Medien als Lernmedien hinaus. Mit einem Lernen über Medien wird in Anlehnung an Ruge $(2014,188)$ die Zielstellung verbunden, «die Entwicklung von Medienkompetenz zu fördern und somit den Lernenden zur Partizipation in einer immer stärker mediatisierten Welt zu befähigen» (ebd.). Medien sind in diesem Verständnis nicht die Mittel der Vermittlung, sondern werden selbst zum Thema bzw. Inhalt der Vermittlungssituation. Bei einer erneuten Betrachtung der Studienordnungen kann strukturell aufgezeigt werden, inwiefern Studierenden auch Möglichkeiten zum Lernen über Medien eröffnet werden. So lässt die zentrale Kompetenzformulierung «Den Einsatz neuer Medien pädagogisch begründen und argumentativ vertreten» (TU Darmstadt 2009, 3) die Interpretation zu, dass eine pädagogische Begründung auf 
inhaltlicher Ebene formuliert werden kann. Das Thema bzw. der Inhalt des Pflichtmoduls «Didaktik, Methodik und Medien» (TU Darmstadt 2009, 3) ist die «Analyse und Reflexion der Bedingungen, Ziele, Inhalte, Formen und Mittel von Unterricht» (ebd.). Mit dieser Präzisierung des Moduls scheint auch die Lesart legitim, Medien als Inhalte des Unterrichts zu thematisieren. Inwiefern Studierenden in der Praxis Möglichkeiten zum Lernen über Medien eröffnet werden, kann gleichwohl nicht explizit beantwortet werden. Vielmehr lässt sich sagen, dass im Rahmen des Pflichtmoduls die Lehrenden der drei Modulveranstaltungen auf Basis der Studienordnung die Legitimation haben, Möglichkeiten zum Lernen über neue Medien zu eröffnen.

Die drei Modulveranstaltungen umfassen laut Studienordnung das Seminar oder die Vorlesung «Allgemeine Didaktik», die Vorlesung «Pädagogik der Neuen Medien» sowie das Seminar «Schulentwicklung». Von diesen drei Veranstaltungen sind von den Studierenden zwei Veranstaltungen zu besuchen. Studierende haben beispielsweise die Option, sich für das Seminar «Schulentwicklung» sowie für das Seminar «Allgemeine Didaktik» zu entscheiden. Im Rahmen dieser Veranstaltung besteht die Möglichkeit, dass Lehrende die Zieldimension «Den Einsatz neuer Medien pädagogisch begründen und argumentativ vertreten [zu können]» (TU Darmstadt 2009, 3) nicht adressieren. Insofern eröffnet die Studienordnung - trotz der Formulierung zentraler Zielkompetenzen - die Option, dass sich Studierende nicht mit dem Einsatz neuer Medien aus einer pädagogischen Perspektive beschäftigen müssen, sofern sich die Lehrenden der Seminare «Allgemeine Didaktik» und "Schulentwicklung» nicht dafür verantwortlich fühlen. Entsprechend hätten diese Studierenden gemäss der Studienordnung auch keine Möglichkeiten zum Lernen über Medien im Rahmen des Pflichtbereichs der Grundwissenschaften.

Das Thema bzw. der Inhalt des Wahlpflichtmoduls «Informationspädagogik» (TU Darmstadt 2009, 6) beschränkt sich auf die Formulierung «Einsatz Neuer Medien in Bildungsprozessen» (ebd.). Wie bereits skizziert, sind Kompetenzziele einerseits so fokussiert formuliert, dass sie Medien vor allem als Mittel verstehen und gerade nicht als Gegenstand. Zugleich finden sich offenere Formulierungen, die auch die Interpretation zulassen, dass Möglichkeiten zum Lernen über Medien zu eröffnen sind. Eine beispielhafte Formulierung ist die bereits zitierte Kompetenzbeschreibung: «für die pädagogische Sinnhaftigkeit selbst entwickelter Formen des Medieneinsatzes argumentativ (auch in Bezug auf kritische Nachfrage) eintreten». Weitere offene Formulierungen lauten «eigene Kenntnisse und Fähigkeiten auf dem Gebiet der Neuen Medien so weitergeben, dass andere davon lernen können» sowie «die Entwicklungen auf dem Gebiet der Neuen Medien so beurteilen, dass daraus der eigene Weiterbildungsbedarf abgeleitet werden kann». Ähnlich wie beim vorherigen Pflichtmodul wird ein Lernen über Medien nicht explizit gefordert. Allerdings eröffnen Formulierungen wie «eigene Kenntnisse und Fähigkeiten auf dem Gebiet der Neuen Medien» die Möglichkeit, Medien in diesem Kontext nicht nur als Mittel zu verstehen, sondern 
selbst zum Lerngegenstand zu erklären. Daraus ergibt sich die Konsequenz, dass Studierende auf Basis der Studienordnung potenziell im Wahlpflichtbereich Möglichkeiten zum Lernen über Medien haben. Daran sind jedoch die Bedingungen geknüpft, dass Studierende sich für diesen Wahlpflichtbereich entscheiden und dass Lehrende der Modulveranstaltungen das Lernen über Medien anregen.

\subsubsection{Problemkonkretisierung und offene Gestaltungsmöglichkeiten}

Bei Betrachtung skizzierter Leitbilder für Medienbildung als institutionelle Vermittlungsaufgabe einerseits und der konkreten curricularen und praxisbezogenen Rahmenbedingungen der Lehrerinnen- und Lehrerbildung an der TU Darmstadt anderseits, kann eine erste Problemdimension konkretisiert werden: Die konkrete Praxis der Lehrerinnen- und Lehrerbildung an der TU Darmstadt bietet zum Zeitpunkt des Forschungs- und Gestaltungsprojektes kaum institutionell verankerte Möglichkeiten alle Lehramtsstudierende auf ihre zukünftigen Aufgaben zur integrativen Vermittlung von Medienkompetenz vorzubereiten. Stattdessen sind die Möglichkeiten, relevante mediendidaktische und -pädagogische Angebote wahrnehmen zu können, abhängig von den gewählten Studien- bzw. Unterrichtsfächern, den jeweiligen Dozentinnen und Dozenten sowie dem Interesse der Lehramtsstudierenden. So war es bis zum Wintersemester 2017/2018 möglich, ein Lehramtsstudium erfolgreich abzuschliessen, ohne eine Lehrveranstaltung mit explizitem oder reflexivem Bezug zu digitalen Medien besucht zu haben ${ }^{79}$. Eine Pflichtveranstaltung für alle Lehramtsstudierenden bzw. eine medienpädagogisch und mediendidaktisch verpflichtende Lehrerinnenund Lehrerbildung existiert zum Zeitpunkt der Studie nicht.

Das entsprechende zukünftige Lehrkräfte gemäss der skizzierten Leitbilder (Kap. 4.2.1) angemessen darauf vorbereitet werden, Medienbildung im Rahmen ihrer Schulfächer integrativ vermitteln zu können, kann in Anlehnung an die Positionen von Kammerl und Ostermann (2010), Niesyto (2012) und Moser (2012) begründet bezweifelt werden. Kammerl und Ostermann $(2010,48)$ skizzieren in ihrer «Expertise zum Stellenwert der Medienkompetenzförderung in Schulen» den «Teufelskreislauf fehlender Medienbildung» (Abb. 4.2). Sie markieren u. a. das Problem, dass Lehramtsstudierende zum Studienbeginn kaum über Medienkompetenzen verfügen und «die meisten von ihnen erfahren», so Kammerl und Ostermann $(2010,48)$, «keine oder kaum Ausbildung in diesem Bereich». Zu einem ähnlichen Schluss kommt Kammerl auch fünf Jahre später: «Medienkompetenzförderung als zentrale Aufgabe schulischer Medienbildung ist nicht im Bewusstsein aller (zukünftigen) Lehrpersonen verankert» (Kammerl 2015, 33). Zugleich sind medienpädagogische Angebote im Lehramtsstudium, wie Kammerl $(2015,33)$ exemplarisch für die Christian-Albrechts-Universität

79 Seit dem Wintersemester 2017/2018 gibt es im neuen Lehramtsstudiengang ein medienpädagogisches Pflichtmodul. 
zu Kiel und die Universität Hamburg zeigt, zwar belegbar aber nicht verpflichtend. Gründe für die wenigen Kompetenzen der Lehramtsstudierenden sind für Kammerl und Ostermann $(2010,48)$ u. a. die eigenen Schulerfahrungen bzw. das Problem, dass Medienbildung in Schulen nicht adäquat realisiert wird. Zugleich reproduzieren jene unvorbereiteten Lehramtsabsolventinnen und -absolventen das Problem fehlender Medienbildung an Schulen, wenn sie über keine angemessene medienpädagogische und mediendidaktische Ausbildung verfügen.

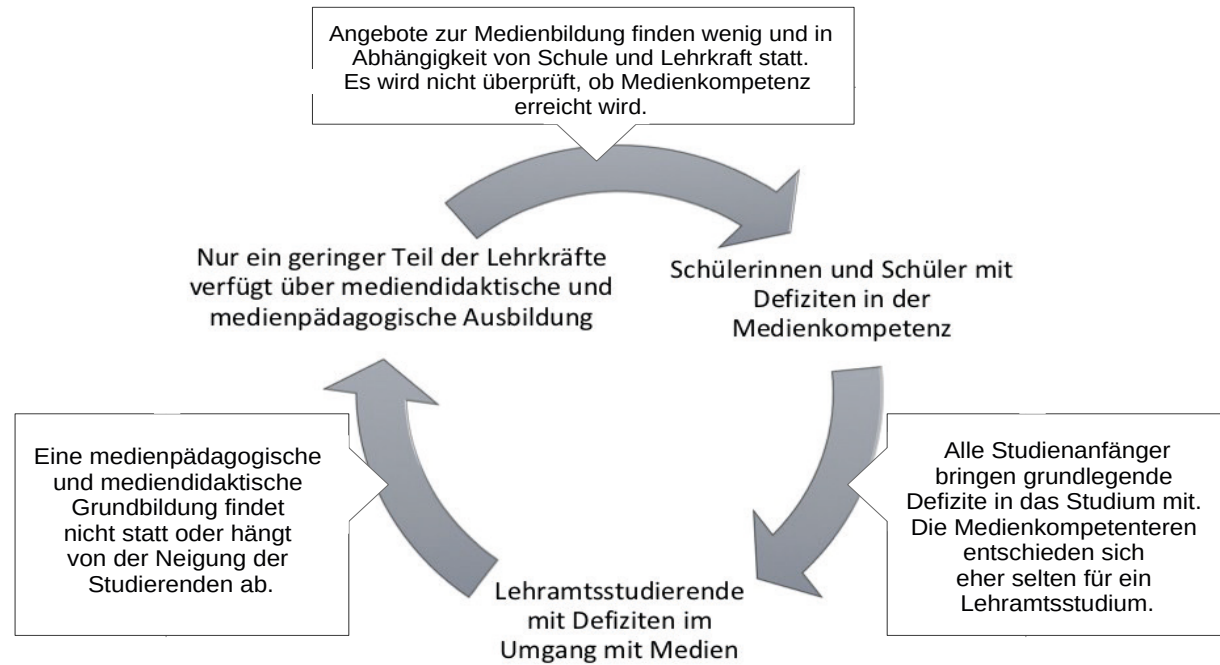

Abb. 4.2: $\quad$ Teufelskreis der Medienbildung nach Kammerl und Ostermann $(2010,48)$.

Ein Ausweg aus diesem Teufelskreislauf sehen Kammerl und Ostermann (2010, 49) in einer "stärkeren Verankerung in den Lehrplänen» sowie einer "Stärkung der Medienbildung in der Lehrerbildung». Zugleich kritisieren sie, dass eine medienpädagogisch und mediendidaktisch verpflichtende Lehrerinnen- und Lehrerbildung in der ersten Phase kaum erfolgt ist. Diese Problematik und eine vergleichbare Lösungsperspektive einer medienpädagogischen Lehrerinnen- und Lehrerbildung diskutiert Niesyto (2012) für das Bundesland Baden-Württemberg. Auch Moser (2012, 267) argumentiert dafür, dass die «Medienbildung in der Ausbildung der Lehrkräfte aller Schulstufen massiv zu verstärken ist». Die notwendige Verstärkung begründet sich für Moser $(2012,267)$ auch aufgrund der aktuellen Situation, dass eine Medienbildung für Lehrkräfte an Hochschulen «nur mit schwachen Anteilen vertreten [ist]». Diese Problematik zeigt sich auch im Fall der TU Darmstadt (Kap. 4.2.2). Zur medienpädagogischen Professionalisierung von Lehramtsstudierenden skizzieren Kammerl und Ostermann (2010, 51 f.) bestehende Überlegungen für ein Mindestcurriculum für die erste Phase der Lehrerinnen- und Lehrerbildung. Zudem verweisen Kammerl und Ostermann $(2010,50)$ auf verschiedene Ansätze zur Konzeptualisierung 
medienpädagogischer Kompetenz von Lehrerinnen und Lehrern, z. B. von Blömeke $(2005)^{80}$.

Zum produktiven Umgang mit dem skizzierten Problem einer fehlenden medienpädagogisch und mediendidaktisch verpflichtenden Lehrerinnen- und Lehrerbildung konnte im Rahmen des vorliegenden Projektes kein Veränderungsprozess des Curriculums angestossen werden. Stattdessen wird ein Ansatz verfolgt, den Kammerl und Ostermann $(2010,45)$ in Anlehnung an Kron und Sofos (2003) für den Unterricht empfehlen: «Integrative Medienbildung als curriculare Transformationsaufgabe». Übertragen auf die Praxis der Hochschullehre im Bereich der universitären Lehrerinnen- und Lehrerbildung scheint diese Perspektive anschlussfähig an ein weites Begriffsverständnis einer integrativer Medienbildung (Kap. 1.1). Wenn digitale Medien im Unterricht, wie in den Leitbildern skizziert (Kap. 4.2.1) sowohl Gegenstand als auch Mittel sein sollen, wird im Folgenden davon ausgegangen, dass diesem Anspruch auch für die universitäre Lehrerinnen- und Lehrerbildung zu folgen ist. Entsprechend gilt es auch in den jeweiligen Studienfächern zu hinterfragen, in welcher Weise Zielsetzungen, Medien, Methoden und Inhalte weiterentwickelt werden können. Zur exemplarischen Planung, Erprobung und empirischen Untersuchung einer integrativen Medienbildung in der Lehrerinnen- und Lehrerbildung dienten ausgewählte Seminare an der TU Darmstadt. Die Seminare zählen zu den erziehungswissenschaftlichen Anteilen des Lehramtsstudiums (Horstkemper 2004) bzw. können im Bereich der Bildungswissenschaften der ersten Phase der Lehrerinnen- und Lehrerbildung verortet werden (KMK 2014). Innerhalb dieses institutionellen Rahmens wird zum einen der Versuch unternommen, den disziplinären und fachwissenschaftlichen Vermittlungsaufgaben gerecht zu werden (KMK 2014; Horstkemper 2004). Zum anderen sollen Möglichkeiten geschaffen werden, um zur medienpädagogischen und mediendidaktischen Professionalisierung beizutragen. Mit dem gewählten Ansatz eröffnen sich spezifische Gestaltungs- und Handlungsmöglichkeiten, welche im Folgenden näher bestimmt werden. Dafür erfolgt eine Verortung im Kontext der Lehrerinnen- und Lehrerbildung sowie hinsichtlich hochschuldidaktischer Handlungsebenen.

Einen systematischen Einblick in den Aufbau der Lehrerinnen- und Lehrerbildung in Deutschland veranschaulicht Reintjes (2006) in Form von Wirkungsketten (Abb. 4.3). In einer bildungsadministrativen bzw. -organisatorischen Perspektive wird in der Darstellung zwischen Inputs, Outputs sowie den dazwischen liegenden Prozessen differenziert. Zudem werden drei Phasen der Lehrerinnen- und Lehrerbildung unterschieden: Die erste Phase als Lehramtsstudium an der Universität, die zweite Phase als Vorbereitungsdienst bzw. Referendariat auf Schule- und Studieneingangsebene

80 Seit der Veröffentlichung von Kammerl und Ostermann (2010) sind zudem weitere Konzepte (z. B. Moser 2010b) und Aktualisierungen bestehender Konzepte entstanden (z. B. Mayrberger 2012a; Tulodziecki 2012). 
sowie die dritte Phase in Form von Fortbildungen und weiteren Angeboten. Die gewählten Seminare an der TU Darmstadt lassen sich als Praxis- und Forschungsfeld in der ersten Phase der Lehrerinnen- und Lehrerbildung verorten. In Betrachtung der schematischen Darstellung der Struktur der Lehrerinnen- und Lehrerbildung in Deutschland (Abb. 4.3) von Reintjes $(2006,83)$ wird erkennbar, dass entsprechende Seminare zwar Wirkungen im Prozess haben können, jedoch zugleich von verschiedenen Bedingungen gerahmt werden. Die Gestaltungsmöglichkeiten zur Veränderung der Praxis sind beispielsweise abhängig von den jeweiligen Studienordnungen der Universitäten sowie übergeordneten Standards zur Lehrerinnen- und Lehrerbildung.

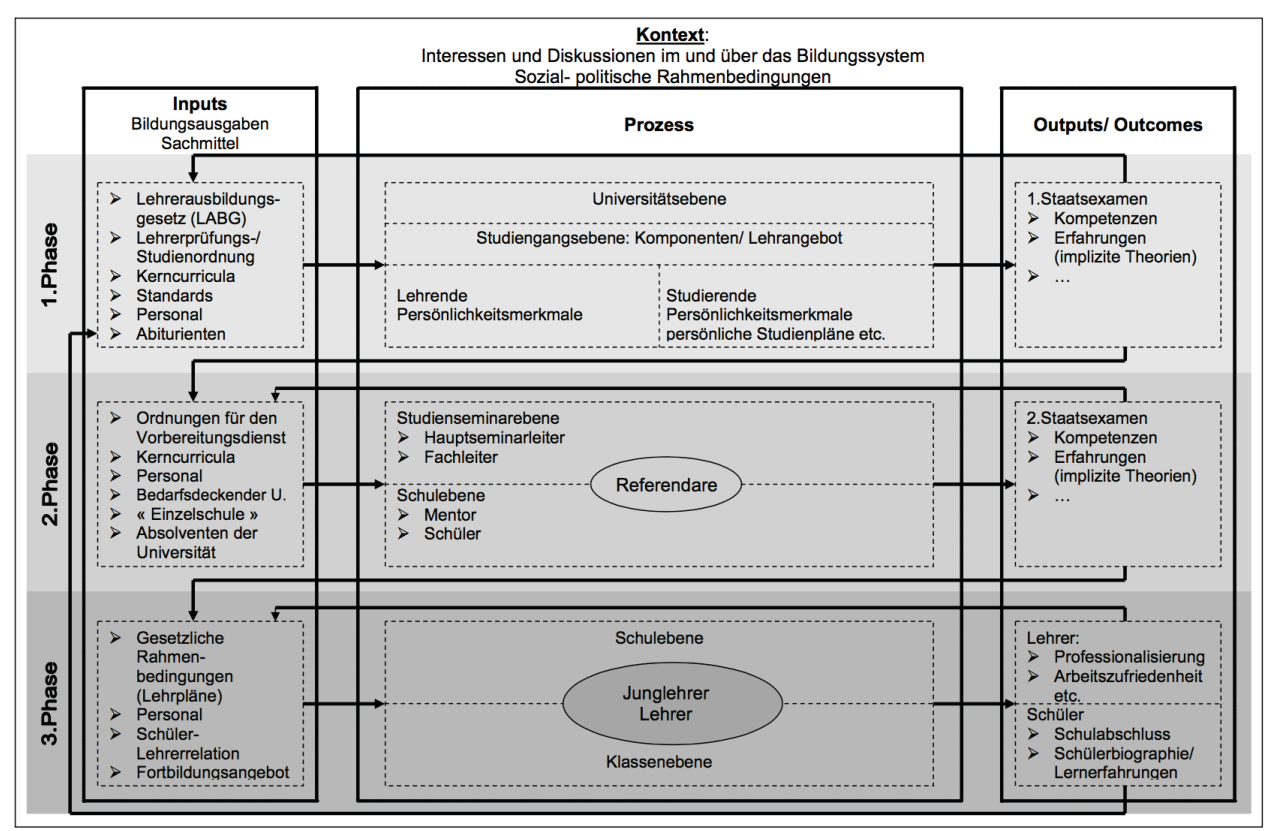

Abb. 4.3: Struktur der Lehrerinnen- und Lehrerbildung in Deutschland von Reintjes $(2006,83)$.

Mit der Unterscheidung der verschiedenen Phasen gehen zudem weitere Unterschiede einher, die u. a. das Verhältnis zwischen «erziehungswissenschaftlichem und curriculumsbezogenem Wissen sowie zwischen Theorie und Praxis» (SchiefnerRohs 2012a, 46) betreffen. Die erste Phase der Lehrerinnen- und Lehrerbildung kann nach Terhart (2000) in vier Studienelemente unterschieden werden: Fachstudien (Terhart 2000, 99 ff.), fachdidaktische Studien (ebd., 102 ff.), erziehungswissenschaftliche Studien (ebd., 104 ff.) und schulpraktische Studien (ebd., 107 ff.). Diese verschiedenen Studienanteile sollen in je unterschiedlicher Weise dazu beitragen, die wissenschaftliche und theoretische Basis für die Entwicklung relevanter Kompetenzen bereitzustellen. Die Aufgabe des erziehungswissenschaftlichen Studiums in der Lehrerinnen- und Lehrerbildung sieht Schiefner-Rohs (2012a, 46) mit Verweis auf den Wissenschaftsrat vor allem in der «wissenschaftlichen Grundlegung» sowie 
der «Entwicklung von Reflexionskompetenz» (Wissenschaftsrat 2001) ${ }^{81}$. Das Praxisund Forschungsfeld kann als spezifischer Ausschnitt der ersten Phase der Lehrerinnen- und Lehrerbildung näher bestimmt werden und es wird zugleich angedeutet, dass weitere normative Leitbilder für entsprechende Veranstaltungen existieren. Mit der empirischen Untersuchung der Praxis können Erkenntnisse gewonnen werden, um die Möglichkeiten und Grenzen einer integrativen Medienbildung im Rahmen erziehungswissenschaftlicher Seminare des Lehramtsstudiums konkreter zu bestimmen $^{82}$.

Weiterhin eröffnen (hochschul-)didaktische Perspektiven die Möglichkeit, das Praxis- und Forschungsfeld hinsichtlich didaktischer Handlungs- und Analyseebenen zu konkretisieren. Als Instrument «für die Strukturierung des komplexen Phänomenzusammenhangs der Didaktik» unterscheiden Kron et al. $(2014,45)$ zwischen vier Betrachtungsebenen: der makrosozialen, der institutionellen, der mikrosozialen sowie der intrapersonalen Ebene (Tab. 4.2). Für Kron et al. $(2014,51)$ sind die mikrosoziale Ebene entscheidend für die Didaktik, wenngleich die vier Betrachtungsweisen «in einem gegenseitigen Verweisungszusammenhang zu sehen [sind]». Mit dem Erkenntnisziel, die Möglichkeiten und Grenzen einer integrativen Medienbildung in Seminaren der erziehungswissenschaftlichen Anteile des Lehramtsstudiums näher bestimmen zu können, lässt sich die Betrachtungsebene des Praxis- und Forschungsfeldes zunächst zwischen der mikrosozialen Ebene und der intrapersonalen Ebene verorten. Hinsichtlich der Frage, wie Menschen mit Medien für Medien sensibilisiert werden können, erscheint es für eine Einschätzung des Anregungsgehalts von Pädagogikseminaren mit Wikibooks sinnvoll, eine Einschätzung der «Lern-, Bildungs-, Entwicklungsprozesse der handelnden Subjekte/Individuen» vorzunehmen. Eine entsprechende Perspektive erscheint anschlussfähig an die von Sesink und Reinmann (2015) formulierte Bedeutungsdimension «Entwicklung als Ereignis und Geschehen» zu betrachten (Kap. 2.3.3).

81 Die zentralen Leitbilder der erziehungswissenschaftlichen Studien in der Lehrerinnen- und Lehrerbildung werden ausführlich im Kapitel 5.2.1 dargestellt.

82 Gleichwohl ist jeweils zu prüfen, inwiefern die Rahmenbedingungen derart ähnlich sind, dass die gewonnenen Erkenntnisse für den jeweils spezifischen Kontext fruchtbar sind, da sich auch die erziehungswissenschaftlichen Studien an unterschiedlichen Universitäten hinsichtlich Umfang und Schwerpunktsetzung unterscheiden. 
(1) Die makrosoziale Ebene

(2) Die institutionelle Ebene

(4) Die intrapersonale Ebene gesellschaftliche Konstitutionsbedingungen regelgeleiteten Handelns; ökonomische, politische, kuturelle, soziale Strukturen und Funktionszusammenhänge

kulturelle und gesellschaftliche Einrichtungen und Organisationen: Betriebe, Universitäten, Schulen, Massenmedien, Kirchen

Interaktion und Kommunikation, interpersonale Beeinflussung: Eltern-Kind-Beziehung, Familie, Unterricht, Peer-Group

Lern-, Bildungs-, Entwicklungsprozesse der handelnden Subjekte/Individuen, Konstitution von Regelbewusstsein, Konstruktion von Wissen, kognitive, emotionale Strukturen, Einstellungen und Erwartungen

Tab. 4.2: Vier Betrachtungsebenen didaktischer Phänomene von Kron et al. $(2014,45)$.

Ein alternatives Modell zur Strukturierung der für die Hochschullehre spezifischen Handlungszusammenhänge bzw. für hochschuldidaktisches Handeln hat Wildt $(2002,7)$ vorgelegt. Ausgehend «von einem curricularen Aufbau von Lehr- und LernZusammenhängen als eine Abfolge von Lehr- bzw. Lernsituationen», unterscheidet Wildt (2002, 7 f.) zwischen sieben «Handlungs- bzw. Analyseebenen»:

1. (Inter)Aktionen sind als «zeitlich begrenzte Aktionen bzw. Interaktionen in Lehrund Lernzusammenhängen» (Wildt 2002, 8) zu verstehen (z. B. «ein Redebeitrag»)

2. (Lern)Situationen sind als «raumzeitliche Einheiten» (ebd.) zu verstehen, in denen die jeweiligen (Inter)Aktionen eingebunden sind (z. B. methodische Arrangements wie «Referate» oder «Gruppenarbeiten»).

3. (Lehr)Veranstaltungen lassen sich als die «Zusammenfassung einander zugeordneter, aufeinander folgender Lehr-Lernsituationen verstehen» (ebd.).

4. Teilstudiengänge (Module) umfassen für Wildt $(2002,8)$ «Lehrveranstaltungen oder Lehrveranstaltungssequenzen», welche abgrenzbare fachliche Handlungszusammenhänge darstellen.

5. Studiengänge bestehen für Wildt $(2002,8)$ aus Modulen und sind auf spezifische Abschlüsse ausgerichtet und zugleich «mehr als die Summe der Module, aus denen sie zusammengesetzt sind».

6. Studiengangsysteme markieren die Zusammenhänge unterschiedliche Studiengänge in Form von «zahlreichen Überschneidungen, Übergängen, aber auch [...] Abgrenzungen voneinander» (ebd.).

7. Systematische Vernetzung markiert die «Verflechtung» der Studiengangsysteme in ihrer Eingebundenheit in gesellschaftliche Zusammenhänge (z. B. Bildungssystem, Beschäftigungssystem) (ebd.).

Auf Basis des Handlungsmodells von Wildt $(2002,7)$ können die Gestaltungs- und Analysemöglichkeiten für das vorliegende Projekt auf drei Ebenen fokussiert werden: «(Lehr)Veranstaltungen», «(Lern)Situationen» und «(Inter)Aktionen». Zugleich 
wird sichtbar, dass die Gestaltungsmöglichkeiten durch Studienmodule, spezifische Studiengänge sowie Studiengangsysteme gerahmt werden. Diese Rahmung entspricht den erziehungswissenschaftlichen Studien für Lehramtsstudierende in der Lehrerinnen- und Lehrerbildung im Allgemeinen sowie dem Studium der Grundwissenschaften an der TU Darmstadt im vorliegenden Fall.

\subsubsection{Zwischenfazit}

Im Rahmen der vorgenommenen Problematisierung erfolgte eine Diskussion aktueller Leitbilder zur institutionellen Medienbildung bzw. zur pädagogisch-strukturierten Medienkompetenzförderung. Für die schulische Medienbildung wurde zum einen die Relevanz dieses Bildungsauftrages als Leitbild hervorgehoben (Kap. 4.2.1.1). Zum anderen wurde skizziert, dass schulische Medienbildung integrativ in den Schulfächern erfolgen und ein Lernen mit sowie ein Lernen über Medien umfassen soll (Kap. 4.2.1.2, 4.2.1.3). Mit diesen Leitbildern sind zugleich verschiedene Implikationen für die Lehrerinnen- und Lehrerbildung verbunden. Allerdings finden sich auf bildungspolitischer Ebene kaum angemessene Leitbilder für die Lehrerinnen- und Lehrerbildung (Kap. 4.2.1.4). An diesen zwei Problemdimensionen wurde verdeutlicht, dass diese Problematik auch bei Betrachtung der Praxis der TU Darmstadt deutlich wird (Kap. 4.2.2.1, 4.2.2.2). Zur Identifikation möglicher Handlungsoptionen wurde die Idee skizziert, Seminare in den erziehungswissenschaftlichen Studien für Lehramtsstudierende neu zu konzipieren. Die damit verbundenen Gestaltungs- und Analysemöglichkeiten wurden hinsichtlich verschiedener Modelle verortet.

Mit diesen Überlegungen sind zugleich verschiedene Fragen und Herausforderungen verbunden, welche in den folgenden Kapiteln näher thematisiert und diskutiert werden. Dafür erfolgt zunächst eine Positionierung zu den Begriffen «Medienbildung» (Kap. 4.3.1), «Medienpädagogische Kompetenz» (Kap. 4.3.2) sowie «soziale Medien» (Kap. 4.3.3). Eine entsprechende Positionierung scheint notwendig, um eine Nachvollziehbarkeit der Begriffsverwendung zwischen fachwissenschaftlichen und bildungspraktischen Diskursen zu ermöglichen. Auf Basis der begrifflichen Klärung widmen sich die zwei folgenden Kapitel dem Forschungsstand zum Lernen mit sozialen Medien in der Hochschule (Kap. 4.4) und es erfolgt eine Betrachtung von sozialen Medien als potenzielles Thema bzw. potenzieller Gegenstand in der Lehre (Kap. 4.5). Die erarbeiteten Erkenntnisse dienen als Orientierung zur Formulierung eines begründeten Entwurfs (Kap. 6). 


\subsection{Begrifflich-theoretische Positionierung}

Mit den folgenden Ausführungen wird das Ziel verfolgt, die bisherigen Begriffsverwendungen sowie deren jeweiligen Begriffsverständnisse zu explizieren und zwischen der medienpädagogischen Praxis der Lehrerinnen- und Lehrerbildung in der Hochschule sowie dem medienpädagogischen Forschungs- und Theoriediskurs zu verorten. Dafür erfolgt in Anlehnung an Jörissen (2011) zunächst eine Differenzierung und Verortung von drei Perspektiven auf den Begriff der Medienbildung (Kap. 4.3.1). Das folgende Kapitel widmet sich der Vorstellung ausgewählter Modelle zum Begriff der medienpädagogischen Kompetenz. Dabei wird zum einen die orientierende Funktion entsprechender Modellierungen hervorgehoben. Zum anderen wird u. a. in Anlehnung an Mayrberger (2012a) und Moser (2010b) diskutiert, wie Modelle zur medienpädagogischen Kompetenz die Charakteristika sozialer Medien berücksichtigen können (Kap. 4.3.2). Abschliessend erfolgt im Rahmen des Kapitels eine Konkretisierung des Begriffs «soziale Medien» und eine Differenzierung hinsichtlich drei unterschiedlicher Vorstellungen entsprechender Medien (Kap. 4.3.3).

\subsubsection{Medienbildung (und Medienkompetenz)}

Der Begriff Medienbildung hat in den letzten Jahren eine zunehmende Verbreitung erfahren. In unterschiedlichen Variationen findet sich der Begriff vor allem in bildungspolitischen und -administrativen Dokumenten ${ }^{83}$ (Kap. 4.2.1). Zudem steht der Terminus «strukturale Medienbildung» für ein bildungstheoretisch fundiertes Konzept der medienpädagogischen Fachdebatte (Jörissen und Marotzki 2009). Mit der Verbreitung und Verwendung des Begriffs über die medienpädagogische Fachdebatte hinaus wird einerseits die Hoffnung verbunden, gesellschaftlich für ein spezifisches Thema zu sensibilisieren und Aufmerksamkeit zu schaffen (Tulodziecki 2010). Anderseits macht Jörissen $(2011,212)$ darauf aufmerksam, dass Begriffe, die in «verschiedenen Diskursen zirkulieren», in verschiedenen «perspektivischen Gefügen adaptiert werden». Trotz der begrifflichen Äquivalenz können mit dem Begriff «Medienbildung» in den verschiedenen Diskursen entsprechend unterschiedliche Aspekte zum Ausdruck gebracht werden. Um Klarheit über das Begriffsverständnis im vorliegenden Projekt zu schaffen, werden in Anlehnung an Jörissen (2011) zunächst drei Bedeutungsdimensionen des Begriffs skizziert: (a) «Medienbildung als Auftrag und Ergebnis von Bildungsinstitutionen» (Kap. 4.3.1.1) und (b) «Medienbildung als Ziel und/oder Resultat individueller Lernprozesse» (Kap. 4.3.1.2) sowie (c) Medienbildung

83 Exemplarisch genannt werden kann an dieser Stelle der Beschluss «Medienbildung in der Schule». In diesem wird Medienbildung von der KMK (2012) zur «Pflichtaufgabe schulischer Bildung» erklärt (Kap. 4.2.1.1). Ein zweites Beispiel stellt die Formulierung «Portfolio Medienbildungskompetenzen» dar. Mit diesem Begriff versucht das hessische Kultusministerium die Kompetenzen von Lehrerinnen- und Lehrer, die für eine Medienkompetenzförderung von Schülerinnen und Schüler notwendig sind, begrifflich zu fassen (Hessischer Bildungsserver 2016). 
als bildungstheoretisches Konzept (Kap. 4.3.1.3). Nach einer Diskussion der Chancen und Grenzen der jeweiligen Perspektiven (Kap. 4.3.1.4) erfolgt eine eigene Positionierung (Kap. 4.3.1.5).

\subsubsection{Medienbildung als Auftrag und Output von Bildungsinstitutionen}

Das Verständnis von Medienbildung als Auftrag und Output von Bildungsinstitutionen kann mit Jörissen $(2011,213)$ auf den «übergreifenden Zusammenhang der administrativen und institutionellen Organisation von Ausbildung im weitesten Sinne» bezogen werden. Bildung lässt sich in diesen Zusammenhang als «die organisierte Bereitstellung von Optionen für Individuen im Interesse des Erwerbs von Wissen und Kompetenzen durch entsprechende gesellschaftliche Maßnahmen [verstehen]» (Jörissen 2011, 213). Dieses begriffliche Verständnis von Bildung «umfasst das Ganze der administrativen Entscheidungen, ihrer Realisierung und der sich daraus ergebenden Effekte» (ebd.). Wird der Begriff Medienbildung in entsprechenden Kontexten verwendet, ist dieser nach Jörissen $(2011,214)$ als ein «übergreifendes, breitbandiges Konzept zu verstehen, das den gesellschaftlichen Bildungsauftrag vor der zeitdiagnostischen Folie einer durchgängig und tiefgreifend mediatisierten Gesellschaft neu bewertet». Einen Einblick in die Frage, wie diese Bewertungen aktuell ausfallen und welche Konsequenzen für den gesellschaftlichen Bildungsauftrag gezogen werden, wurde bereits im Rahmen der Problematisierung skizziert (Kap. 4.2.1). Für Jörissen (2011) wird mit diesem Begriffsverständnis eine bildungspolitische und administrative Perspektive auf Medienbildung zum Ausdruck gebracht.

Über den bildungspolitischen Diskurs hinaus macht Jörissen (2011) darauf aufmerksam, dass über die Entwürfe und die Diskussion von Bildungsstandards für Medienbildung ein entsprechendes begriffliches Verständnis zum Gegenstand der medienpädagogischen Fachdebatte wird. Dafür verweist Jörissen $(2011,214)$ auf die Beiträge von Moser (2010a), Tulodziecki (2010) sowie Herzig und Grafe (2010). Zur Irritation von Jörissen (2011) verwenden sie den Begriff der Medienbildung nicht im Sinne eines bildungstheoretisch fundierten Konzeptes. Vielmehr verstehen Tulodziecki (2010) sowie Herzig und Grafe (2010), wie sie in einer weiteren Publikation deutlich machen, «die Medienbildung als zusammenfassenden Begriff für alle bildungsrelevanten Prozesse mit Medienbezug mit der Zielstellung eines bestimmten Kompetenzniveaus» (Tulodziecki et al. 2010, 178). Für Jörissen (2011) knüpfen sie damit an das begriffliche Verständnis einer schulischen Medienbildung an bzw. an die Logik einer bildungspolitischen bzw. -administrativen Perspektive. Dabei lässt sich das von Herzig und Grafe (2010) entwickelte Konzept der «Bildungsstandards in der Medienbildung» aus der Sicht von Jörissen $(2011,214)$ als «struktureller Koppler der Erfolgskriterien des Bildungswesens (Output) mit denen pädagogischer Praxis (individuelle Lernerfolge)» verstehen. Ferner markiert Jörissen $(2011,228)$ die Chance, dass «die 
Medienbildung» in Sinne dieses Begriffsverständnis als «struktureller Koppler fachintern begründeter Notwendigkeiten einerseits und bildungspolitischer sowie administrativer Kommunikationsbedarfe andererseits fungieren» kann.

\subsubsection{Medienbildung als Ziel und/oder Resultat individueller Lernprozesse}

Ein zweites Verständnis von Medienbildung kann in Anlehnung an Jörissen (2011, $215 \mathrm{ff}$.) als Ziel und/oder Resultat individueller Lernprozesse auf Basis pädagogischer Vermittlungsarbeit verstanden werden. Ein entsprechendes Verständnis steht einerseits in Relation zu der zuvor skizzierten bildungspolitischen und -administrativen Perspektive. Anderseits muss es jedoch auf einer anderen Ebene verortet werden. Statt «als abstrakter «Output) des Bildungssystems» steht für Jörissen $(2011,215)$ das aufgrund von pädagogischen Handlungen (vorläufig) erzielte Ergebnis individueller Lernprozesse im Fokus dieses Begriffsverständnisses. In dieser Perspektive lässt sich Bildung gemäss Jörissen verstehen als «[...] das Verfügen eines Individuums über ein (jeweils zu bestimmendes) von ihm erworbenes Wissen und Können auf einem jeweils zu bestimmenden Niveau, das je nach Kontext von einfachen Kenntnissen bis hin zu komplexen und reflexiven Kompetenzen reichen kann» (Jörissen 2011, 216).

Dieser Logik folgend liegt für Jörissen $(2011,218)$ der Schluss nahe, den Begriff der Medienbildung als äquivalent bzw. als Synonym zum Medienkompetenzbegriff zu verstehen. Während Bildung als Ergebnis individueller Lernprozesse markiert wurde, wird der Begriff der Medienbildung als das Ergebnis individueller Lernprozesse mit Medienbezug verstanden. Ein entsprechendes Verständnis ähnelt, so Jörissen (2011), stark dem Begriff von Medienkompetenz wie er beispielsweise von Sutter (2010) definiert wird. Für Sutter $(2010,43)$ werden mit dem Begriff Medienkompetenz «vielfältige Fähigkeiten und Fertigkeiten beschrieben und zwar überwiegend in Form statisch feststellbarer Lernresultate». Medienbildung kann in diesem Sinne als das «individuelle Pendant zu ‘der Medienbildung〉 als abstrakter Output des Bildungssystems» (Jörissen 2011, 218) verstanden werden. Im Folgenden wird ein entsprechendes Begriffsverständnis als pädagogisch-praktische Perspektive bezeichnet.

Medienbildung wird aus dieser Perspektive, so Jörissen $(2011,218)$, «nah an formalen pädagogischen Vermittlungsprozessen diskutiert». Als Beispiel verweist Jörissen $(2011,218)$ auf den Begriff der «Mediengrundbildung». Dieser wird - analog zum Begriff der «Grundbildung» - als «die Vermittlung von Medienkompetenzen verstanden». Medienbildung als Ergebnis (medien-)pädagogischer Vermittlungsarbeit zu verstehen, erfolgt nach Jörissen $(2011,229)$ überwiegend im Kontext «des etablierten Konzepts der ‘Medienkompetenz»». Zugleich finden sich im Kontext von Praxisprojekten Beispiele, in denen die Begriffe Medienkompetenz und Medienbildung synonym verwendet werden. So skizziert Aufenanger (2003) im Rahmen eines Projektberichtes in einem ausserschulischen Kontext, "dass die Vermittlung von 
Medienkompetenz - bzw. von Medienbildung, wie im Projekt genannt - auch für jüngere Kinder möglich ist» (Aufenanger 2003, 6). Während Aufenanger (2003, 1 ff.) in seiner Argumentation konsequent den Medienkompetenzbegriff verwendet, greift er in der Darstellung des Praxisprojektes auf das Vokabular des Projektpartners zurück: «Das von der Stiftung des Ravensburger Verlags geförderte Projekt war angetreten, Kindern in Horteinrichtungen Medienbildung zu vermitteln» (Aufenanger 2003, 7).

\subsubsection{Medienbildung als bildungstheoretisches Konzept}

Das dritte Verständnis von Medienbildung fokussiert Bildungsprozesse «im Kontext von Medialität» (Jörissen 2011, 222). Dieses von Jörissen (2011, 222 f.) vorgestellte Begriffsverständnis bezieht sich auf den wissenschaftlichen Ansatz der «strukturalen Medienbildung» (Jörissen und Marotzki 2009). Im Gegensatz zu den zuvor vorgestellten Begriffsverständnissen und Perspektiven wird dabei explizit auf eine Bildungstheorie Bezug genommen. So basiert dieses Verständnis nach Jörissen $(2011,223)$

«[...] auf einer strukturalen Bildungstheorie, die Bildungsprozesse als eine Form komplexer, selbstreflexiver Lern- und Orientierungsprozesse versteht. Bildung lässt sich aus dieser Perspektive nicht als Ergebnis oder Zustand verstehen, sondern muss als ein Prozess aufgefasst werden, in welchem vorhandene Strukturen und Muster der Weltaufordnung durch komplexere Sichtweisen auf Welt und Selbst ersetzt werden» (Jörissen 2011, 223).

Mit dem Begriff der Medialität weist Jörissen darauf hin, dass es in Abgrenzung zu den vorherigen Begriffsverständnissen theoretisch nicht ausreichend ist, Medien als Werkzeuge oder Instrumente zu konzeptualisieren. Jörissen $(2011,222)$ sieht die «konstitutiven Aspekte von 〈Medien`» in diesem Begriffsverständnis in ihrer «immanente[n] Strukturiertheit». Diese Strukturiertheit von Medien lässt sich nicht, so Jörissen $(2011,222)$ weiter, «auf der Oberfläche ihres Erscheinens» erschliessen. In einer Reihe weiterer Negativbestimmungen macht Jörissen $(2011,222)$ darauf aufmerksam, dass bekannte Dichotomien zur Beschreibung der Auseinandersetzung von Menschen mit Medien, beispielsweise «Nutzer (Subjekt) vs. Angebot (Medium)», für den Ansatz der «Strukturalen Medienbildung» keine hinreichend komplexe Beschreibung ermöglichen. Im Zusammenhang des zuvor markierten Bildungsbegriffs wird von einer «tiefgreifenden Verwobenheit von Subjektivität und Medialität» (Jörissen 2011, 223) ausgegangen.

Ohne die Komplexität der begrifflich-theoretischen Zusammenhänge an dieser Stelle ausreichend reproduzieren zu können, wurden erste Indizien markiert, dass sich die Verwendung der Begriffe «Bildung» und «Medien» deutlich von den zwei zuvor skizzierten Begriffsverständnissen unterscheiden. So überrascht es nicht, dass Jörissen (2011) die vorgestellte Perspektive von bildungspolitischen und 
praxisbezogenen Bildungsverständnissen abgrenzt und den zugrunde liegenden Bildungsbegriff im «Diskurs der modernen Bildungstheorie» (Jörissen 2011, 220) verortet. Im Gegensatz zu den vorherigen Perspektiven markiert das Begriffsverständnis der strukturalen Medienbildung eine dezidiert bildungstheoretische Perspektive. Wenngleich Jörissen $(2011,231)$ in seinem Beitrag explizit auf seinen Ansatz der strukturalen Medienbildung eingeht, steht dieser Ansatz exemplarisch für «ein ganzes Spektrum vorhandener, wenn auch vielfach erst ansatzweise ausdifferenzierter Medienbildungsmodelle, die sich je nach Art und Gewichtung ihrer Bildungs- und Medialitätstheorien unterscheiden». Möglichkeiten zur exemplarischen Veranschaulichung bildungstheoretischer Perspektiven bietet gemäss Jörissen $(2011,231)$ die «bildungstheoretisch motivierte Theoriediskussion um Medienbildung». Dafür verweist Jörissen $(2011,231)$ u. a. auf die Beiträge des sechsten Jahrbuchs Medienpädagogik von Meder (2007), Sesink (2007) sowie Spanhel (2007), welche die Vielfalt bildungstheoretisch argumentierender Ansätze erkennbar werden lassen ${ }^{84}$.

\subsubsection{Kritik der jeweiligen Perspektiven}

In Anlehnung an Jörissen (2011) lassen sich verschiedene Möglichkeiten und Grenzen der skizzierten Begriffsverständnisse markieren ${ }^{85}$. Mit der Fokussierung einer administrativ-organisatorischen Perspektive weist Jörissen $(2011,213)$ auf das Problem hin, "dass Vorstellungen von Bildung vorhanden sind, nicht aber, welche» (ebd.). Ferner können (und müssen) Fragen wie «pädagogische Vermittlungsprozesse zu gestalten sind» (ebd.) oder was Bildung überhaupt meint, so Jörissen (2011, 213), in dieser Perspektive nicht berücksichtigt werden. Mit der Beschreibung von «Medienbildung» durch Bildungsstandards sieht Jörissen $(2011,215)$ zudem die Gefahr, dass diese Beschreibung nicht auf einen «konsistente[n] und theoretisch wohlbegründeten Fachbegriff von Bildung» aufbauen muss. Ausführlicher werden die Grenzen, aber auch die Chancen von Bildungsstandards für die Medienbildung von Tulodziecki (2010) diskutiert. Dafür markiert Tulodziecki (2010) aus seiner Sicht

84 Diese Vielfalt zeigt sich u. a. daran, dass Spanhel (2007) Medienbildung zwar als Schlüsselbegriff sieht, im Gegensatz zu Jörissen (2011) diesen jedoch nicht nur prozessbezogen versteht. In einem weiten Verständnis lässt Medienbildung nach Spanhel $(2007,48)$ als «Voraussetzung und Ziel einer Teilhabe der Menschen aller Altersstufen an den Kommunikationsprozessen in der heutigen Mediengesellschaft begreifen» (ebd.). In einem engen Verständnis ist Medienbildung für Spanhel $(2007,48)$ «zu verstehen als Prozess und als Ergebnis des Prozesses der Vermittlung von Welt und Selbst durch Medien».

85 Als Vertreter der strukturalen Medienbildung ist diese Kritik von Jörissen (2011) nicht überraschend. Zudem ist anzumerken, dass die Kritik von Jörissen (2011) im Spannungsfeld zwischen Wissenschaft und Praxis deutlich im Bereich der scientific community verortetet werden muss (Kap. 2.1). Wenn Jörissen (2011) in der Diskussion von Medienbildungsstandards beispielsweise auf das Problem hinweist, dass die Berücksichtigung unterschiedlicher gesellschaftlicher Gruppen die Gefahr birgt, keinen theoretisch fundierten Bildungsbegriff zu verwenden, markiert er damit ein Problem, dass die Wissenschaft mit der Praxis hat. Inwiefern die wissenschaftlich fragwürdige Theoriefundierung von Medienbildungsstandards ein Problem für die Praxis darstellt, wird von Jörissen (2011) nicht in den Blick genommen. 
verschiedene Spannungsfelder, die letztlich nicht auflösbar sind, aber eine Positionierung ermöglichen. Dabei beschränkt Tulodziecki $(2010,82)$ seine Einschätzung von Medienbildungsstandards nicht auf die wissenschaftliche Fachdebatte, wie es bei Jörissen (2011) der Fall ist. So eröffnen Bildungsstandards für Tulodziecki (2010, 82) die Chance, zur Verständigung von unterschiedlichen Gruppen beizutragen und den «Geltungsanspruch der Medienbildung in der Bildungsdiskussion insgesamt [zu] stärken». Ferner benennt Tulodziecki $(2010,82)$ die folgenden Funktionen, die Medienbildungsstandards erfüllen können, «z. B. eine Orientierungs-, eine Curriculums-, eine Reform-, eine Qualifizierungs- und eine Evaluationsfunktion» (ebd.).

In Abgrenzung von der pädagogisch-praktischen Perspektive ist es für die wissenschaftliche Fachdebatte nach Jörissen $(2011,219)$ keine gute Entscheidung, die Begriffe Medienbildung und Medienkompetenz synonym zu verwenden. Diese eingenommene Position begründet Jörissen (2011, 218 f.) u. a. hinsichtlich der Differenzen von kompetenztheoretischen und bildungstheoretischen Überlegungen. So ermöglicht eine kompetenztheoretische Perspektive für Jörissen (2011, 218 f.) zwar die Beschreibung eines Dispositionsaufbaus, nicht aber einer «Dispositionstransformation». Insbesondere zur Betrachtung von «Orientierungswissen» ist es für Jörissen $(2011,219)$ jedoch notwendig, prozessorientierte und transformative (bzw. bildungstheoretische) Modelle zu verwenden. Jörissen $(2011,220)$ problematisiert entsprechend, dass die Gleichsetzung der Begriffe Medienbildung und Medienkompetenz "an den begrifflich-theoretischen Differenzen beider Modelle vorbeigehen und insofern beiden Konzepten nicht gerecht [werden können]». Diese Problematik wird u. a. auch von Tulodziecki et al. (2010) und Mayrberger (2012a) markiert. So sehen Tulodziecki et al. $(2010,178)$ keine Veranlassung dafür, «den Begriff der Medienkompetenz zugunsten des Begriffs der Medienbildung aufzugeben». Der Auffassung von Tulodziecki et al. (2010) folgend, sind die Begriffe «Medienkompetenz und Medienbildung» nach Mayrberger (2012a, 403) als unterschiedliche Diskussionsbereiche medienbezogener Aneignung anzusehen, die sich langfristig betrachtet bedingen (können)».

Die Potenziale der bildungstheoretischen Perspektive sieht Jörissen (2011, 227) darin, «(medien-)kulturelle Transformationen von Bildungsoptionen und Subjektivierungsbedingungen sichtbar bzw. reflexiv zugänglich zu machen und diese somit in erziehungswissenschaftlichen, aber auch darüber hinausgreifende Selbstverständigungsdiskurse einzubringen». Darüber hinaus können Erkenntnisse der Medienbildungsforschung dazu beitragen, so Jörissen (2011, 228), «qualifizierte Reflexionsangebote» für medienpädagogische Handlungsfelder zu entwickeln. Ferner betont Jörissen (2011) die Potenziale für den medienpädagogischen Diskurs und markiert u. a. die Möglichkeiten zur «Ausdifferenzierung der theoretischen Fundamente der Medienpädagogik» sowie der «Erweiterung ihrer methodologischen-forschungsbezogenen Ansätze». Wenngleich Jörissen (2011) darauf verzichtet die Grenzen dieser 
Perspektive zu markieren, lassen sich diese im Vergleich mit den vorherigen Begriffsverständnissen implizit bereits erkennen. So stehen bildungsorganisatorische. bzw. -administrative Fragen und Themen nicht im Fokus dieser Perspektive. Ebenfalls ausgeblendet werden praktisch-pädagogische Fragen wie beispielsweise normative Fragen zur Gestaltung medienpädagogischer Praxis.

\subsubsection{Zwischenfazit und Positionierung}

Zur Klärung und Diskussion des Begriffs der Medienbildung wurden mit Bezug auf Jörissen (2011) drei verschiedene Perspektiven bzw. Begriffsverständnisse unterschieden (Kap. 4.3.1.1, 4.3.1.2, 4.3.1.3) und die Möglichkeiten und Grenzen der jeweiligen Perspektive skizziert (Kap. 4.3.1.4). Unterschiede zeigten sich dabei hinsichtlich des jeweils zugrunde liegenden Bildungsbegriffs sowie bei der Verortung zu bestimmten Diskurszusammenhängen. Ferner markieren die jeweiligen Begriffsverständnisse spezifische Blickwinkel auf den Zusammenhang zwischen Lern- und Bildungszusammenhängen in einer zunehmend mediatisierten Welt, wodurch jeweils spezifische Zusammenhänge sichtbar gemacht bzw. ausgeblendet werden.

Bei Betrachtung der bisherigen Begriffsverwendung zur Problematisierung der vorhandenen Leitbilder (Kap. 4.2.1) sowie der universitären Praxis (Kap. 4.2.2) wurde Medienbildung überwiegend aus einer bildungspolitischen bzw. -administrativen Perspektive diskutiert. An dieses Verständnis anknüpfend, wird der Begriff Medienbildung nach Tulodziecki et al. $(2010,178)$ «als zusammenfassender Begriff für alle bildungsrelevanten Prozesse mit Medienbezug mit der Zielstellung eines bestimmten Kompetenzniveaus» verstanden. Dieses Verständnis erscheint kompatibel zu der Beschreibung von Jörissen (2011, 214), welcher Medienbildung aus einer bildungsadministrativen Perspektive als «breitbandiges Konzept» zur Realisierung des skizzierten Bildungsauftrages zusammenfasst (Kap.4.2.1.1). Es dient für das vorliegende Projekt als Begriffsverständnis für den Medienbildungsbegriff.

Den Argumentationen von Jörissen (2011), Tulodziecki et al. (2010) und Mayrberger (2012a) folgend, ist der Medienkompetenzbegriff deutlich geeigneter, die zu formulierenden Ziele von Lehraktivitäten und Ergebnisse individueller Lernprozesse zu beschreiben. Bezugnehmend auf Tulodziecki $(1997,116)$ kann Medienkompetenz dabei konkret verstanden werden als die «Fähigkeit und Bereitschaft zu einem sachgerechten, selbstbestimmten, kreativen und sozial verantwortlichen Handeln in einer von Medien mitgestalteten Welt». Daran anknüpfend wird für die Planung und Realisierung medienpädagogischer und mediendidaktischer Lehr- und Lernaktivitäten der Begriff der medienpädagogischen Kompetenz verwendet. Unter dem Begriff werden in Anlehnung an Tulodziecki (2012) und Moser (2010b) Wissensbestände, Fähigkeiten sowie Handlungs- und Lernbereitschaft gefasst und im folgenden Kapitel näher präzisiert (Kap. 4.3.2). Mit der Formulierung «Medienbildung für 
Lehrerinnen- und Lehrer» sind in der vorliegenden Arbeit entsprechend der zuvor skizzierten Begriffsverständnisse alle bildungsrelevanten Prozesse mit Medienbezug in der Lehrerinnen- und Lehrerbildung mit dem Ziel, Studierende bei der Entwicklung medienpädagogischer Kompetenzen zu fördern und zu unterstützen, zu verstehen.

\subsubsection{Medienpädagogische Kompetenz als normative Orientierungshilfe}

In der Problematisierung wurde anhand bildungspolitischer Dokumente die Relevanz schulischer Medienbildung betont und konkrete Leitbilder und Zielvorstellungen skizziert (Kap. 4.2.1). Auffällig ist jedoch, dass sich in der bildungspolitischen und -administrativen Diskussion kaum zugehörige Leitbilder für die Lehrerinnenund Lehrerbildung finden (Kap. 4.2.1.4). Die Frage, welche Kompetenzen Lehrkräfte in einer digital geprägten bzw. zunehmend mediatisierten Welt benötigen, um professionell handeln zu können, scheint in der öffentlichen Diskussion kaum Beachtung zu finden. Es werden jedoch Antworten auf diese Fragestellung benötigt, um Lehrerinnen- und Lehrer angemessen auf ihre Aufgaben im Kontext schulischer Medienbildung vorbereiten zu können. Um einen Einblick in den medienpädagogischen Fachdiskurs zu diesem Thema zu eröffnen, widmen sich die folgenden Ausführungen dem Konstrukt der medienpädagogischen Kompetenz. Ausgehend von den Überlegungen von Blömeke $(2000,2005)^{86}$ wurde das Konstrukt in den letzten Jahren für forschungs- und praxisbezogene Zusammenhänge diskutiert und weiterentwickelt (z. B. Mayrberger 2012a; Tulodziecki 2012; Schiefner-Rohs 2012b). Exemplarisch werden dafür drei Ansätze vorgestellt:

- der Entwurf eines Kompetenz-Standard-Modells für die erste Phase der Lehrerinnen- und Lehrerbildung von Tulodziecki (2012) (Kap. 4.3.2.1),

- die Weiterentwicklung des Teilkonstruktes der mediendidaktischen Kompetenz nach Mayrberger (2012a) (Kap. 4.3.2.2) sowie

- die Formulierung von Standards zur Medienkompetenz für Lehrerinnen- und Lehrer nach Moser (2010a) (Kap. 4.3.2.3)

Die vorgestellten Überlegungen bieten mit unterschiedlichen Akzentuierungen erste Orientierungshilfen zur Formulierung und Förderung medienpädagogischer Zielstellungen für die Lehrerinnen- und Lehrerbildung und werden im Rahmen des Entwurfs (Kap. 6) erneut aufgegriffen. Die Ausführungen von Tulodziecki (2012)

86 Das Konstrukt der medienpädagogischen Kompetenz zielt nach Blömeke $(2005,77)$ «auf die Wahrnehmung medienbezogener Aufgaben durch Pädagogen, insbesondere durch Lehrpersonen». In den Fokus wird dabei folgende Sichtweise gerückt: «medienbezogene Aufgaben stellen also der Einsatz von Medien beim Unterrichten und die Realisierung von medienbezogenen Erziehungsaufgaben dar» (Blömeke 2005, 77). Dafür lassen sich nach Blömeke $(2005,78)$ fünf Kompetenzbereiche unterscheiden: (1.) «medienerzieherische Kompetenz», (2.) «mediendidaktische Kompetenz», (3.) «sozialisationsbezogene Kompetenz im Medienzusammenhang», (4.) «eigene Medienkompetenz» sowie (5.) «Schulentwicklungskompetenz im Medienzusammenhang». 
und Mayrberger (2012a) knüpfen dabei an die Arbeiten von Blömeke (2000, 2005) an. Tulodziecki (2012) entwirft ein Kompetenzmodell-Standard-Modell, welche sich an den Zielen der ersten Phase der Lehrerinnen- und Lehrerbildung orientiert und zugleich ausgewählte Kompetenzbereiche des Konstruktes medienpädagogischer Kompetenz berücksichtigt. Mayrberger (2012a) widmet sich der Aktualität des Teilkonstruktes «mediendidaktischer Kompetenz» und entwirft einen Erweiterungsvorschlag dieses Kompetenzbereichs. Als alternatives Modell zur medienpädagogischen Kompetenz wird als dritter Ansatz der Entwurf von Moser (2010a) zur Formulierung von Standards zur Medienkompetenz für Lehrerinnen- und Lehrer skizziert.

\subsubsection{Kompetenz-Standard-Modell für die Lehrerinnen- und Lehrerbildung von Tulodziecki}

Das von Tulodziecki $(2012,294)$ entworfene «Kompetenz-Standard-Modell» kann als Versuch verstanden werden, "positive Funktionen von Standards» hervorzuheben und Umsetzungsschwierigkeiten möglichst zu verringern. Neben der Herleitung der Standards auf Basis des Kompetenzmodells von Blömeke (2000) sowie hinsichtlich spezifischer Leitideen der universitären Lehrerinnen- und Lehrerbildung wurde dafür auch die Entwicklung des Modells hinsichtlich der getroffenen Entscheidungen präsentiert. Dabei geht Tulodziecki $(2012,293)$ grundsätzlich davon aus, dass die Standards dann positiv in der Praxis wirken, wenn diese «als Reflexionshilfen und als Anlässe für eine diskursive Verständigung zwischen Lernenden und Lehrenden dienen». In diesem Zusammenhang betont Tulodziecki $(2012,293)$ auch, dass es zur Realisierung der Standards auch wichtig ist, dass eine Verständigung «über die Prozessqualitäten des Studiums erfolgt» (ebd.). Ferner sieht Tulodziecki $(2012,294)$ in dem vorgelegten Kompetenz-Standard-Modell die Chance, «Möglichkeiten der Förderung» aufzuzeigen. So sollten die Überlegungen zur Differenzierung von Niveaustufen «den Entwicklungscharakter von Kompetenzen betonen und zugleich auf Förderungsmöglichkeiten verweisen» (Tulodziecki 2012, 294).

Tulodziecki $(2012,282)$ unterscheidet zur Strukturierung seines «KompetenzStandard-Modell» für die erste Phase der Lehrerinnen- und Lehrerbildung zwischen Kompetenzbereichen und Kompetenzaspekten ${ }^{87}$. In Vereinfachung der Dimensionen von Blömeke (2005) sind für Tulodziecki $(2012,282)$ drei medienpädagogische Handlungsbereiche für die Schule zentral, welche im Folgenden als

87 Der Begriff des Kompetenzbereiches wurde u. a. mit Verweis auf die KMK (2004) verwendet. Kompetenzbereiche werden von Tulodziecki $(2012,282)$ auch als «zentrale medienpädagogische Handlungsbereiche» bezeichnet und markieren unterscheidbare Zielbereiche, für die Lehrkräfte Kenntnisse und Fähigkeiten benötigen. Der Begriff des Kompetenzaspektes wird von Tulodziecki (2012) nicht explizit definiert. Auf Basis seines Modells zeigt sich, dass Kompetenzaspekte abhängig von den Leitbildern spezifischer Bildungsinstitutionen sind und quer zu den Kompetenzbereichen liegen. Zudem versteht Tulodziecki $(2012,286)$ im Verlauf des Textes «den Ausprägungsgrad von Kompetenzaspekten im Sinne potenzieller Entwicklungen». 
Kompetenzbereiche bezeichnet werden: «die Mediennutzung zur Anregung und Unterstützung von Lernprozessen ${ }^{88}$, «die Wahrnehmung von medienbezogenen Erziehungs- und Bildungsaufgaben ${ }^{89}$ sowie "die Entwicklung medienpädagogischer Konzepte in der Schule» ${ }^{90}$ (Tulodziecki 2012, 282). In Betrachtung der zentralen Aufgaben der universitären Lehrerinnen- und Lehrerbildung unterscheidet Tulodziecki (2012, 282 ff.) Kompetenzaspekte, welche sich an übergeordneten Leitideen der spezifischen Phase der Lehrerinnen- und Lehrerbildung orientieren. Das «wesentliche Ziel der universitären Lehrerbildung», besteht für Tulodziecki (2012) darin, «dass die Studierenden wissenschaftliche Grundlagen für die berufliche Handlungsfähigkeit erwerben». Für den ausgewählten Kompetenzbereich «Mediennutzung zur Anregung und Unterstützung von Lernprozessen» lassen sich die von Tulodziecki (2012) unterschiedenen fünf Kompetenzaspekte exemplarisch veranschaulichen (Tab. 4.3).

\begin{tabular}{|c|c|}
\hline Kompetenzaspekt & Standard \\
\hline $\begin{array}{l}\text { Bedingungen für medi- } \\
\text { enpädagogisches Han- } \\
\text { deln durchschauen und } \\
\text { einschätzen }\end{array}$ & $\begin{array}{l}\text { A1.1: Die Bedeutung der ausserschulischen Mediennutzung für das } \\
\text { Lernen mit Medien unter Bezug auf theoretische Ansätze und } \\
\text { empirische Ergebnisse an Beispielen beschreiben. } \\
\text { A.1.2: Konsequenzen aus der Bedeutung gemäß A1.1 an Beispielen } \\
\text { für das Lernen mit Medien demonstrieren. }\end{array}$ \\
\hline $\begin{array}{l}\text { Theoretische Ansätze } \\
\text { für medienpädagogi- } \\
\text { sches Handeln charak- } \\
\text { terisieren und bewerten }\end{array}$ & $\begin{array}{l}\text { A2.1: Lerntheoretische und mediendidaktische Ansätze sowie empi- } \\
\text { rische Ergebnisse zum Lernen mit Medien sachgerecht darstel- } \\
\text { len. } \\
\text { A2.2: Lerntheoretische und mediendidaktische Ansätze zum Lernen } \\
\text { mit Medien aus empirischer, normativer und realisierungsbezo- } \\
\text { gener Sicht bewerten. }\end{array}$ \\
\hline $\begin{array}{l}\text { Beispiele für medienpä- } \\
\text { dagogisches Handeln } \\
\text { analysieren und be- } \\
\text { werten }\end{array}$ & $\begin{array}{l}\text { A3.1: Unterrichts- oder Projektbeispiele mit Medienverwendung } \\
\text { hinsichtlich ihrer Zielvorstellungen, Lernvoraussetzungen, Lehr- } \\
\text { handlungen und Lernaktivitäten [...] analysieren. } \\
\text { A3.2: Unterrichts- oder Projektbeispiele mit Medienverwendung aus } \\
\text { empirischer, normativer und realisierungsbezogener Sicht be- } \\
\text { werten. }\end{array}$ \\
\hline
\end{tabular}

88 Dieser Kompetenzbereich ist vergleichbar mit dem Teilkonstrukt der «mediendidaktischen Kompetenz», welche Blömeke $(2005,77)$ als Fähigkeit beschreibt, «Medien in geeigneten Lehr-Lernformen reflektiert zu verwenden».

89 Dieser Kompetenzbereich ist vergleichbar mit dem Teilkonstrukt «medienerzieherische Kompetenz» von Blömeke $(2005,77)$.

90 Dieser Kompetenzbereich ist vergleichbar mit dem Teilkonstrukt «Schulentwicklung» von Blömeke $(2005,77)$. 


\begin{tabular}{|c|c|}
\hline Kompetenzaspekt & Standard \\
\hline $\begin{array}{l}\text { Eigene Vorschläge für } \\
\text { medienpädagogisches } \\
\text { Handeln theoriegeleitet } \\
\text { entwickeln }\end{array}$ & $\begin{array}{l}\text { A4.1: Vorhandene Medienangebote hinsichtlich ihrer angenom- } \\
\text { menen Lernvoraussetzungen sowie ihrer Zielvorstellungen, } \\
\text { Inhalte, methodischen Implikationen, medialen Gestaltungs- } \\
\text { merkmalen und lerntheoretischen Annahmen analysieren } \\
\text { sowie hinsichtlich ihrer Eignung für einen geplanten Unterricht } \\
\text { bewerten. } \\
\text { A4.2: Unterrichts- oder Projektbeispiele mit Medienverwendung mit } \\
\text { Bezug auf theoretische Ansätze entwerfen und hinsichtlich ihrer } \\
\text { Lernvoraussetzungen, Zielvorstellungen, Lehrhandlungen und } \\
\text { Lernaktivitäten ([...]) skizzieren. } \\
\text { A4.3: Für einen geplanten Unterricht einen eigenen Medienbeitrag } \\
\text { oder eine Lernumgebung entwerfen. } \\
\text { A4.4: Eigene Unterrichts- oder Projektbeispiele mit Medienverwen- } \\
\text { dung unter Berücksichtigung empirischer, normativer und } \\
\text { realisierungsbezogener Aspekte reflektieren. }\end{array}$ \\
\hline $\begin{array}{l}\text { Theoriebasierte Beispie- } \\
\text { le für medienpädago- } \\
\text { gisches Handeln erpro- } \\
\text { ben und evaluieren }\end{array}$ & $\begin{array}{l}\text { A5.1: Ein theoriebasiertes Unterrichts- oder Projektbeispiel mit Medi- } \\
\text { enverwendung in einer realen oder simulierten Situation erpro- } \\
\text { ben und für ausgewählte Fragen Daten aufnehmen. } \\
\text { A5.2: Aufgenommene Daten auswerten und interpretieren und die } \\
\text { Erprobung hinsichtlich eines Vergleichs von Planung und Um- } \\
\text { setzung sowie hinsichtlich methodologischer Fragen von Evalu- } \\
\text { ationen reflektieren. }\end{array}$ \\
\hline
\end{tabular}

Tab. 4.3: Kompetenz-Standard-Modell von Tulodziecki (2012, 290 f.) zur Medienpädagogik in der ersten Phase der Lehrerinnen- und Lehrerbildung für den Kompetenzbereich: «Mediennutzung zur Anregung und Unterstützung von Lernprozessen».

Vor dem Hintergrund der formulierten Problematisierung (Kap. 4.2.3) erscheint der Entwurf von Tulodziecki (2012) dahingehend überraschend, dass Tulodziecki (2012) einerseits betont, dass er die eigene Medienkompetenz von Lehrerinnen- und Lehrer als wichtigen Bestandteil des Konstruktes der medienpädagogischen Kompetenz versteht. Anderseits jedoch die eigene Medienkompetenz von Lehramtsstudierenden als Kompetenzbereich in seinem Modell nicht berücksichtigt ${ }^{91}$. Für das vorliegende Projekt sind die Überlegungen von Tulodziecki (2012) unabhängig davon interessant, weil sie spezifisch für die erste Phase der Lehrerinnen- und Lehrerbildung formuliert wurden. So kann das Modell als wissenschaftliche Reflexionshilfe für die vorgenommene Problematisierung der Praxis (Kap. 4.2.2) verwendet werden. Bei erneuter Betrachtung der herausgearbeiteten Probleme (a) «Begrenzte Möglichkeiten zum Lernen mit digitalen Medien» (Kap. 4.2.2.1) und (b) «Lernen über Medien nur als Wahloption» (Kap. 4.2.2.2) zeigt sich beispielsweise, dass viele Studierende keine Anlässe und institutionell verankerten Möglichkeiten haben, um die formulierten $\mathrm{Ni}$ veaustufen zu erreichen. Welche Prozessqualität die vorhandenen Angebote haben,

91 Diese Reduktion begründet Tulodziecki (2012) u. a. mit der Annahme, dass die Medienkompetenz von Studierenden als Voraussetzung für ein Lehramtsstudium betrachtet werden kann. In Anlehnung an den skizzierten «Teufelskreis der Medienbildung» (Kap. 4.2.1.4) von Kammerl und Ostermann (2010) ist diese Annahme jedoch skeptisch zu bewerten. 
wurde dabei noch nicht berücksichtigt. Zum anderen eröffnet das Modell auch eine erste Orientierungsmöglichkeit für den vorzulegenden Entwurf (Kap. 6). Neben den Hinweisen zur Unterscheidung kognitiver Niveaus bietet auch die Strukturierung von Kompetenzaspekten und Kompetenzbereichen eine Orientierungsmöglichkeit zur Formulierung medienpädagogischer Zielstellungen für unterschiedliche Lehrveranstaltungen.

\subsubsection{Mediendidaktische Kompetenz nach Mayrberger}

Bei Betrachtung des Teilbereiches der mediendidaktischen Kompetenz diskutiert Mayrberger (2012a) in ihren Überlegungen die Aktualität des Konstruktes der medienpädagogischen Kompetenz. Die Frage nach der Aktualität stellt Mayrberger (2012a, 391) zum einen bei der Betrachtung «des sich gegenwärtig vollziehenden Medienwandels und der sich damit verändernden (medienbezogenen) Sozialisationsbedingungen». Diese Veränderungen skizziert Mayrberger (2012a, 392) im Zusammenhang mit der «Diskussion um das sich verändernde, allgegenwärtige Internet [...] mit seinen spezifischen Angeboten und Umgangsweisen und deren Bedeutung für Schule und Unterricht heute». Zum anderen kritisiert sie die bestehende Konzeptualisierung mediendidaktischer Kompetenz hinsichtlich der zu starken Fokussierung der «Perspektive der Lehrenden als Akteure im Lehr- und Lernprozess, wenn relativ eng von der Integration von Medien als Werkzeug und Lehr- bzw. Lernmittel im Unterricht gesprochen wird». In Erweiterung der Überlegungen von Blömeke $(2000,2005)$ sowie als Alternative zu dem zuvor skizzierten Kompetenz-Standard-Modell (Kap. 4.3.2.1) von Tulodziecki (2012) werden nach Mayrberger (2012a, 391) heuristische und normative Überlegungen präsentiert, welche als Anregungen zur Entwicklung eines theoretisch-begründeten Entwurfs (Kap. 6) dienen.

Ein zentraler Ausgangspunkt der Überlegungen von Mayrberger (2012a, 395) lautet, dass eine «zeitgemäße didaktische Perspektive auf die Gestaltung von Lehr- und Lernumgebungen mit digitalen Medien» u. a. danach fragen sollte,

«[...] inwieweit die Mediensozialisation der Schülerinnen und Schüler einen Einfluss auf die Integration von digitalen Medien im Unterricht hat, inwieweit sich entsprechende technologische und soziale Potenziale des Social Webs für ein verändertes Lehren und Lernen in formalen Kontexten adaptieren liessen und wie sich Schule und Unterricht grundlegend verändern müssen, damit digitale Medien hier ihr Potenzial zur Unterstützung von Veränderungsprozessen einbringen könne» (Mayrberger 2012a, 395).

Diesen Anspruch an eine didaktische Perspektive begründet Mayrberger (2012a) hinsichtlich drei zentraler Aspekte: einem veränderten «Lehren und Lernen mit dem Social Web» (395 ff.), dem «Partizipativen Lernen» (397 ff.) sowie der «Entgrenzung 
von Lehr- und Lernprozessen» (399 ff.). Gründe für die Weiterentwicklung des Konstruktes liegen für Mayrberger (2012a, 397) hinsichtlich des ersten Aspektes «nicht in den neuen technologischen Möglichkeiten, die das veränderte Netz mit der Social Software heute bietet, denn die sind morgen schon wieder ganz andere». Stattdessen betont Mayrberger (2012a, 397) die «kultivierenden Umgangsweisen mit heutigen digitalen Medien, wie Produktion, Artikulation, Netzwerkbildung oder Partizipation», die für die Weiterentwicklung des Konstruktes mediendidaktischer Kompetenz von Bedeutung sind. Die «soziale Weiterentwicklung des Internets zum Web 2.0», wie sie von Mayrberger (2012a, 397) benannt wird, ist aus ihrer Perspektive anschlussfähig an pädagogische Überlegungen zum Konzept des partizipativen Lernens und markiert den zweiten zentralen Aspekt. Damit ist keine neue Idee gemeint, sondern es werden u. a. Anschlussmöglichkeiten zur konstruktivistischen Didaktik sowie zum ELearning-2.0-Diskurs skizziert. Partizipatives Lernen beschreibt Mayrberger (2012a, 397) dabei als «Beteiligung der Lernenden bei der Gestaltung von individuellen und gemeinsamen Lernprozessen». Durch die Verwendung bzw. durch die Integration digitaler Medien kann, so argumentiert Mayrberger (2012a, 399), "[d]iese Art des Lernens und die Etablierung einer entsprechenden Lernkultur [...] sehr gut realisiert und vielfältiger gestaltet werden». Nur «folgerichtig» ist es für Mayrberger (2012a, 399) in diesem Zusammenhang, mithilfe sozialer Medien zur Öffnung von Schule und Unterricht sowie zur Entgrenzung von Lehr- und Lernprozessen beizutragen. Im Fokus ihres dritten Argumentes stehen $u$. a. die mit sozialen Medien verbundenen Möglichkeiten für «öffentliches Lernen, zu dem von Dritten aktiv beigetragen werden könnte» (Mayrberger 2012a, 399). Um diese Potenziale realisieren zu können, müssen sich Lehrerinnen- und Lehrer darüber bewusst sein, so (Mayrberger 2012a, 401), "dass formale Lernumgebungen so offen gestaltet sein sollten, dass sie (zumindest) Phasen der Selbststeuerung, -bestimmung oder -organisation im Sinne einer konstruktivistisch orientierten Auffassung vom Lehren und Lernen zulassen». Diese offene Gestaltung erfordert von Lehrkräften die Fähigkeit, die Grenzen formale Lernumgebungen erkennen zu können sowie die Bereitschaft, durch soziale Medien «eröffnende Wege» zu erkunden (Mayrberger 2012a, 401). Dabei geht Mayrberger (2012a, 401) davon aus, dass das Verfügen «über zeitgemäße, medienpädagogische Kompetenzen» das «Beschreiten von diesen (zum Teil) neuen Wegen» erleichtert.

Als Vorschlag für eine zeitgemässe Konzeption des Teilbereichs mediendidaktischer Kompetenz adaptiert Mayrberger (2012a, 401) zunächst zwei Inhaltsfelder von Blömeke (2000): «1) Einsatz von Medien und Informationstechnologien als Werkzeug und Mittel im Unterricht» und «2) die Gestaltung und Weiterentwicklung schulischer Lehr- und Lernformen» gemäss der zuvor skizzierten Überlegungen. Zudem erscheint es Mayrberger (2012a) sinnvoll, einen dritten Inhaltsbereich zu ergänzen: «Entgrenzung formaler Lehr- und Lernprozesse mit digitalen Medien» (Mayrberger 2012a, 404). Dabei geht es Mayrberger (2012a, 404) insbesondere darum aufzuzeigen, 
dass die beschriebenen Veränderungen nicht nur Veränderungen von Werkzeugen darstellen. Vielmehr bedarf es auch einer Reflexion des bisherigen Verständnisses «von Unterricht und Schule sowie der Rollen von Lehrenden und Lernenden» (ebd.). Der Vorschlag zur Konzeption der Mediendidaktischen Kompetenz umfasst nach Mayrberger (2012a, 404) entsprechend bestehende Qualifikationsziele und erweitert diese um weitere Zielstellungen (Tab. 4.4).

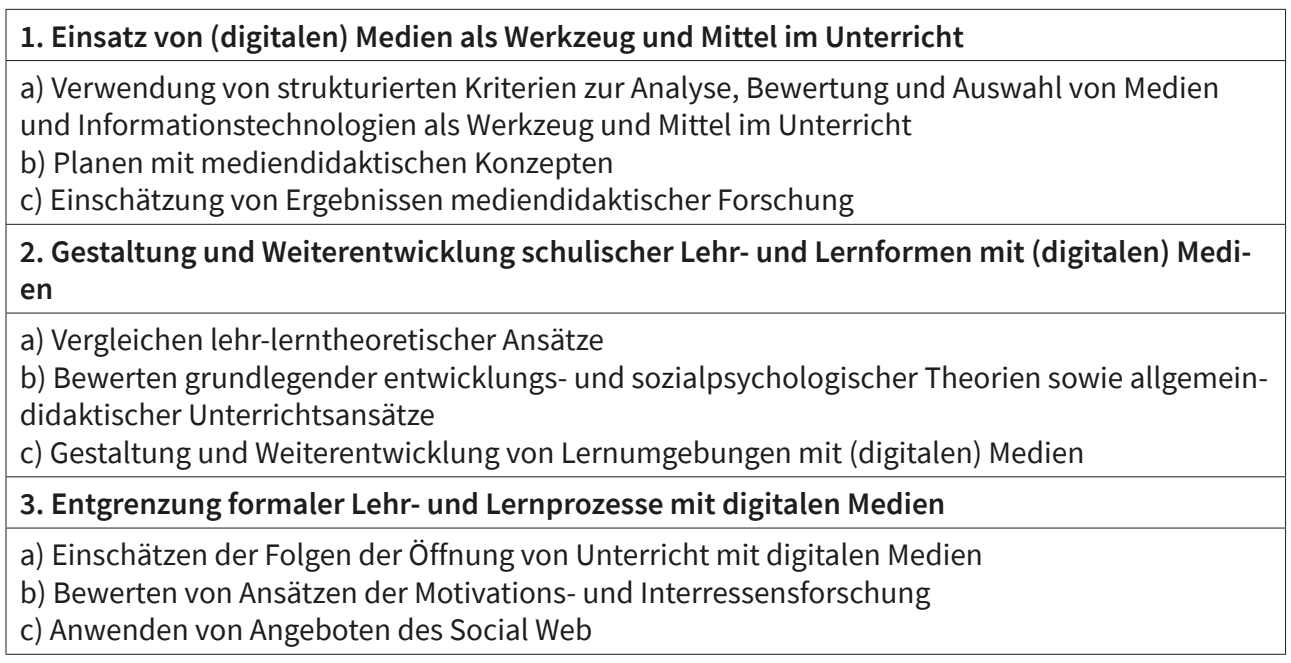

Tab. 4.4: Inhaltsbereiche und Kompetenzen der mediendidaktischen Kompetenz von Lehrenden von Mayrberger (2012a, 401).

Mit dem formulierten Vorschlag unternimmt Mayrberger (2012a, 400 f.) den Versuch, eine Orientierung für eine mittel- bis kurzfristige «Anpassung der medienbezogenen Kompetenzförderung von Lehrenden in allen Phasen der Lehrerbildung» zu eröffnen. Da Mayrberger (2012a) ihre Überlegungen nicht auf eine spezifische Phase der Lehrerinnen- und Lehrerbildung beschränkt - anders als in den vorherigen Überlegungen von Tulodziecki (2012) - bleiben ihre Kompetenzbeschreibungen abstrakter. Zugleich finden sich in den zuvor skizzierten Überlegungen verschiedene inhaltliche Konkretisierungen ihrer Kompetenzen. Beispielsweise markiert sie mit den Begriffen der Partizipation und des partizipativen Lernens zentrale Leitideen zur Weiterentwicklung schulischer Lehr- und Lernformen. Zudem betont sie, dass die Integration aktueller digitale Medien nicht angemessen mit dem Werkzeugbegriff gefasst werden kann und mediendidaktische Kompetenz darüber hinaus geht:

«Mediendidaktisch kompetente Lehrende sind in der Lage, (digitale) Medien im Unterricht unter Ausschöpfung deren spezifischer, didaktischer Potenziale für die Gestaltung von schülerorientierten Lernumgebungen zu verwenden und zu reflektieren sowie die Folgen der Integration digitaler Medien für das formale und informelle Lernen abzuschätzen, kritisch zu bewerten und mit der Entgrenzung umzugehen» (Mayrberger 2012a, 406). 
Der Vorschlag zur Konzeption einer «[m]ediendidaktische[n] Kompetenz von Lehrerinnen und Lehrern» (Mayrberger 2012a, 401) bietet eine weitere Orientierungsmöglichkeit für den vorzulegenden Entwurf eines Pflichtseminars (Kap. 6). Im Vergleich mit dem Vorschlag von Tulodziecki (2012) zum Kompetenzbereich «Mediennutzung zur Anregung und Unterstützung von Lernprozessen» sind insbesondere die Ausführungen zur Entgrenzung sowie zum partizipativen Lernen eine interessante Anregung. In Übereinstimmung mit Tulodziecki (2012) markiert auch Mayrberger (2012a) die Relevanz einer strukturierten medienpädagogischen Professionalisierung.

\subsubsection{Medienkompetenz für Lehrerinnen- und Lehrer nach Moser}

Auch Moser (2010b) widmet sich der Frage, welche Kompetenzen für Lehrerinnenund Lehrer hinsichtlich einer professionellen Medienbildung zentral sind. Anders als die zwei vorherigen Ansätze von Tulodziecki (2012) und Mayrberger (2012a) orientiert sich Moser (2010b) als Ausgangspunkt für seine Überlegungen nicht am Kompetenzmodell zur medienpädagogischen Kompetenz von Blömeke $(2000,2005)^{92}$. Zur Beantwortung der Fragestellung entwirft Moser «Standards der Medienkompetenz» für Lehrkräfte. Dieser Modellentwurf orientiert sich an den «allgemeinen Standards der Lehrerbildung, wie sie an den pädagogischen Hochschulen der Schweiz formuliert sind» (Moser 2010b, 28). Die von Moser bezeichneten «Standards der Medienkompetenz» setzen allgemeine Kompetenzen der Lehrerinnen- und Lehrerbildung voraus und lassen sich als medienspezifische Erweiterungen verstehen. In der Terminologie von Blömeke (2005) lassen sich entsprechende Kompetenzen auch als Dimensionen medienpädagogischer Kompetenz beschreiben. Dabei versucht Moser (2010b, 29) mit seinen Standards «Durchschnittskompetenzen» zu formulieren, «die in Zukunft von allen Lehrern erreicht werden müssten, um erfolgreich im Informationszeitalter unterrichten zu können». Moser (2010b) unterscheidet zehn Bereiche:

- «Fachspezifisches Wissen und Können im Umgang mit Medien» (ebd., 29 f.)

- «Lernen, Denken und Entwicklung» (ebd., 30 f.),

- «Motivation und Interesse an Medien» (ebd., 31),

- «Heterogenität» (ebd., 32),

- «Kooperation, Partizipation und Web 2.0» (ebd., 32 f.),

- «Kommunikation» (ebd., 33 f.),

- «Unterrichten mit Medien» (ebd., 34 f.),

92 Zudem richtet sich die zugehörige Publikation «Schule 2.0 - Medienkompetenz für den Unterricht» von Moser (2010b) im Gegensatz zu den Beiträgen von Tulodziecki (2012) und Mayrberger (2012a) nicht explizit an die scientific community in Form einer wissenschaftlichen Tagung bzw. eines Tagungsbandes. Vielmehr scheint das Taschenbuch primär für Akteure in Schulen geschrieben zu sein. In der Gegenüberstellung von Praxis und scientific community (Kap. 2.2) sind die Publikation von Moser (2010b) zwischen diesen zwei Polen zu verorten. 
- «Diagnose und Evaluation» (ebd., 35),

- «Medien und Gesellschaft» (ebd., 36) und

- «Wissensmanagement» (ebd., 36 f.).

Beim Vergleich mit den zuvor skizzierten Überlegungen von Blömeke (2005), Mayrberger (2012a) und Tulodziecki (2012) finden sich verschiedene Gemeinsamkeiten sowie unterschiedliche Akzentuierungen. Ähnlich wie Blömeke (2005) formuliert Moser (2010b) seine Kompetenzdimensionen ${ }^{93}$ unter Berücksichtigung allgemeiner Leitbilder bzw. Standards für die Lehrerinnen- und Lehrerbildung. Dabei beschränkt sich Moser (2010b) nicht auf die Aufgabenbereiche des Unterrichtens und Erziehens, wie z. B. bei Blömeke (2005), sondern skizziert auch Kompetenzformulierungen für das individuelle und kooperative Wissensmanagement von Lehrerinnen- und Lehrer sowie die Zusammenarbeit mit Kolleginnen und Kollegen sowie mit Eltern. Entsprechend nachvollziehbar erscheint die höhere Anzahl unterschiedlicher Kompetenzdimensionen. Ferner bieten die Ausführungen von Moser (2010b) eine weitere Strukturierungsmöglichkeit der benannten Kompetenzdimensionen. Diese werden jeweils in «Wissen», «Lern- und Umsetzungsbereitschaft» sowie «Können» differenziert ${ }^{94}$. Am Beispiel der Kompetenzdimension «Kooperation, Partizipation und Web 2.0» kann diese Differenzierung exemplarisch veranschaulicht werden (Tab. 4.5).

93 Der Begriff der Kompetenzdimension von Moser (2010b) ist vergleichbar mit dem Begriff des Kompetenzbereichs bei Tulodziecki (2012).

94 Auch Tulodziecki (2012) markiert einen Unterschied zwischen Fertigkeiten und der Bereitschaft von Lehrenden. Allerdings werden diese Überlegungen in seinem Kompetenz-Standard-Modell nicht weiter ausgeführt. 


\begin{tabular}{|l|l|l|}
\hline Wissen & $\begin{array}{l}\text { Lern- und Umsetzungsbereit- } \\
\text { schaft }\end{array}$ & Können \\
\hline $\begin{array}{l}\text { Die Lehrpersonen kennen die } \\
\text { Konzepte der Vernetzung und } \\
\text { Globalisierung, die mit der } \\
\text { Kooperation im Rahmen der } \\
\text { digitalen Medien und des Web } \\
\text { 2.0 verbunden sind. }\end{array}$ & $\begin{array}{l}\text { Die Lehrperson ist bereit, } \\
\text { Verantwortung für eine Lern- } \\
\text { kultur zu übernehmen, in der } \\
\text { Online-Medien und Präsenz- } \\
\text { unterricht eng miteinander } \\
\text { verknüpft sind. }\end{array}$ & $\begin{array}{l}\text { Die Lehrperson unterstützt } \\
\text { Schüler, digitale Medien für } \\
\text { partizipatives Lernen einzuset- } \\
\text { zen (z. B. in der Nutzung web- } \\
\text { basierter Anwendungen, in der } \\
\text { Einrichtung von Lerngruppen } \\
\text { als Social Communities). }\end{array}$ \\
\hline $\begin{array}{l}\text { Sie sind sich der Bedeutung } \\
\text { dieser neuen Kooperationsfor- } \\
\text { men für die Kommunikation } \\
\text { und Kooperation bewusst. }\end{array}$ & $\begin{array}{l}\text { Sie ist sich bewusst, wann } \\
\text { digitale Medien in der Kom- } \\
\text { munikation mit Kollegen und } \\
\text { Eltern einzubeziehen sind. }\end{array}$ & $\begin{array}{l}\text { Sie reflektiert die Kooperation } \\
\text { regelmässig mit ihren Schü- } \\
\text { lern. }\end{array}$ \\
\hline $\begin{array}{l}\text { Sie kennen die Diskussion um } \\
\text { das Verhältnis von medialen } \\
\text { und „realen“ Elementen der } \\
\text { Kommunikation. }\end{array}$ & $\begin{array}{l}\text { Sie ist sich der besonderen } \\
\text { Bedingung bewusst, welche } \\
\text { mit der Kommunikation und } \\
\text { Kooperation über Medien } \\
\text { verbunden sind. }\end{array}$ & $\begin{array}{l}\text { Sie fördert Kooperation mit } \\
\text { Kollegen, Eltern und allen } \\
\text { an Schulen Beteiligten über } \\
\text { Medien (E-Mails, Blogs, News- } \\
\text { letters, etc.). }\end{array}$ \\
\hline \multicolumn{2}{|l}{} & $\begin{array}{l}\text { Sie versteht Lernen als Teil } \\
\text { einer Netzkultur, die nicht nur } \\
\text { passiv rezipiert, sondern aktiv } \\
\text { mit eigenen Beiträgen parti- } \\
\text { zipiert. }\end{array}$ \\
\hline
\end{tabular}

Tab. 4.5: Darstellung der Durchschnittskompetenzen für die Kompetenzdimension «Kopperation, Partizipation und Web 2.0» von Moser (2010b, 33 f.).

In den Kompetenzformulierungen von Moser (2010b, 33 f.) zur Dimension «Kooperation, Partizipation und Web 2.0» (Tab. 4.5) finden sich verschiedene Anknüpfungspunkte zu den Überlegungen von Mayrberger (2012a) zur Aktualisierung der mediendidaktischen Kompetenz. Der Begriff des «partizipativen Lernens» wird sowohl von Mayrberger (2012a) als auch von Moser (2010b) hervorgehoben. Der Verwendung von sozialen Medien bzw. Angeboten des Social Web wird dabei jeweils das Potenzial zugesprochen, zur Veränderung einer Lernkultur beizutragen. Wenngleich die konkreten Standards von Moser (2010b) mitunter Fragen aufwerfen ${ }^{95}$, eröffnet die Strukturierung seines Konstruktes hinsichtlich der zehn Kompetenzdimensionen sowie der Unterscheidung zwischen Wissen, Können sowie einer Lern- und Umsetzungsbereitschaft hilfreiche Anregungen zur Formulierung und Gestaltung medienpädagogischer Lehrerinnen- und Lehrerbildung. Insbesondere seine Ausführungen zur Partizipation und zum Web 2.0 werden in den folgenden Kapiteln näher betrachtet (Kap. 4.5.2.1).

95 Am Beispiel der ausgewählten Dimension «Kooperation, Partizipation und Web 2.0» (Tab. 4.5) stellt sich zum Beispiel die Frage, warum hinsichtlich des Aspektes «Lern- und Umsetzungsbereitschaft» der Operator des Wissens bzw. des «Bewusstseins» gewählt wurde. Diese Formulierung findet sich auch als Operator für den Aspekt des Wissens. 


\subsubsection{Positionierung}

Ausgehend vom Konstrukt der medienpädagogischen Kompetenz von Blömeke $(2000,2005)$ wurden drei ausgewählte Ansätze skizziert, um medienpädagogisch relevante Kompetenzen für Lehrerinnen- und Lehrer zu beschreiben. Das KompetenzStandard-Modell von Tulodziecki (2012) fokussiert die erste Phase der Lehrerinnenund Lehrerbildung und umfasste drei Kompetenzbereiche sowie fünf Kompetenzaspekte (Kap. 4.3.2.1). Die Ausführungen von Mayrberger (2012a) widmen sich der Erweiterung des Teilkonstruktes der mediendidaktischen Kompetenz für alle Phasen der Lehrerinnen- und Lehrerbildung. Dabei werden insbesondere die Herausforderungen für Lehrerinnen- und Lehrer in Auseinandersetzung mit sozialen Medien markiert (Kap. 4.3.2.2). Als dritter Ansatz wurden Überlegungen von Moser (2010b) zur Formulierung von «Standards der Medienkompetenz» von Lehrerinnen- und Lehrer vorgestellt. Mit der von Moser vorgenommen Differenzierung zwischen «Können», «Wissen» sowie «Lern- und Umsetzungsbereitschaft» eröffnete sich eine weitere Orientierungsmöglichkeit zur Formulierung von Kompetenzen als relevante Zielstellungen. Zudem konnten bei Moser (2010b) Anknüpfungspunkte zu den Überlegungen von Mayrberger (2012a) identifiziert werden, welche die Bedeutung sozialer Medien hervorheben.

Mit der Darstellung der verschiedenen Positionen wurde zum einen das Ziel verfolgt, einen ersten Einblick in den medienpädagogischen Fachdiskurs zu eröffnen. Während Sesink (2015) in der ersten Phase einer entwicklungsorientierten Bildungsforschung insbesondere die Problematisierung der Praxis aus einer praxisbezogenen Perspektive betont, wurden in den skizzierten Prozessstandards gestaltungs- und entwicklungsorientierte Forschung (Kap. 2.2) auch die Relevanz des Projektes für den aktuellen Forschungsdiskurs markiert. Diesbezüglich finden sich in den Beiträgen von Tulodziecki (2012), Mayrberger (2012a) sowie Moser (2010b) jeweils Hinweise darauf, dass die medienpädagogische Professionalisierung in der Lehrerinnen- und Lehrerbildung ein relevantes Praxis- und Forschungsfeld darstellt.

Zum anderen erfolgte die Auseinandersetzung mit den drei ausgewählten Modellen mit dem Ziel, normative Orientierungshilfen für die Planung eigener Lehrveranstaltungen zu erarbeiten (Kap. 6). Während in bildungspolitischen und -administrativen Dokumenten nur selten explizit relevante Kompetenzen für Lehrkräfte formuliert werden, eröffnen die skizzierten Modelle strukturierte Zieldimensionen und -bereiche für die medienpädagogische Lehrerinnen- und Lehrerbildung. Für das vorliegende entwicklungsorientierte Forschungsprojekt sind die vorgestellten Überlegungen insbesondere für den Entwurf und die Reflexion der konkreten Praxis interessant. Hinsichtlich der transitiven bzw. gestalterischen Bedeutungsdimension von Entwicklung (Kap. 2.3.3.1) bieten die Überlegungen ein breites Antwortspektrum hinsichtlich der Frage, für welches Ziel «etwas» zu entwickeln ist. Für die Planung und Durchführung des vorliegenden Projektes wurde entschieden, den Kompetenzbereich 
«Mediennutzung zur Anregung und Unterstützung von Lernprozessen» (Tulodziecki 2012) bzw. das Teilkonstrukt der mediendidaktischen Kompetenz (Mayrberger 2012a; Blömeke 2000, 2005) näher zu diskutieren. Zudem wurde in Anlehnung an die Argumentation von Mayrberger (2012a) und dem exemplarisch skizzierten Kompetenzbereich «Kooperation, Partizipation und Web 2.0» von Moser (2010b) die Entscheidung getroffen, die Auseinandersetzung mit sozialen Medien zu fokussieren. Dafür erfolgt zunächst eine begriffliche Klärung der bereits benannten Termini «Social Software», «Social Web», «Web 2.0» und «soziale Medien» (Kap. 4.3.3). Darauf aufbauend werden in den zwei folgenden Kapiteln theoretische sowie empirische Erkenntnisse zur Gestaltung von Hochschullehre mit sozialen Medien diskutiert (Kap. 5.4) und das Thema Partizipation im Zusammenhang mit sozialen Medien konkretisiert (Kap. 5.5).

\subsubsection{Soziale Medien als aktueller Sammelbegriff}

Mit den Begriffen «soziale Medien» oder «Social Web» wird der Versuch unternommen, Entwicklungen und Phänomene des Internets bzw. des World Wide Web begrifflich zu fassen. Neben dem Terminus «soziale Medien» lassen sich sowohl im öffentlichen als auch im sozial- und bildungswissenschaftlichen Diskurs eine Vielzahl weiterer Begriffe identifizieren. Exemplarisch genannt werden an dieser Stelle «Web 2.0», «Social Software», «Read/Write Web» und «Two-way Web» (z. B. Dron 2007; Danciu und Grosseck 2011; Wheeler 2009). Neben der Vielzahl an Begrifflichkeiten finden sich zudem eine Vielzahl an kritischen Diskussionen von und Auseinandersetzungen mit entsprechenden Begrifflichkeiten ${ }^{96}$. Ohne an dieser Stelle die unterschiedlichen Diskussionen der verschiedenen Termini im Detail nachzuzeichnen, zielen die folgenden Ausführungen auf eine begriffliche Präzisierung des Gegenstandes dieses Kapitels. Auf Basis von Taddicken und Schmidt (2017) wird dafür zunächst eine Arbeitsdefinition von sozialen Medien vorgestellt. Im zweiten Schritt wird in Anlehnung an Grell und Rau (2014) eine Differenzierung von drei unterschiedlichen Kategorien von sozialen Medien vorgeschlagen. Die Differenzierung erscheint hilfreich ${ }^{97}$, um die folgenden Ausführungen über die Lern- und Bildungspotenziale sowie mögliche Spannungsfelder in der Bildungspraxis adäquat einschätzen zu können.

Die Arbeitsdefinition orientiert sich im Folgenden am Begriffsverständnis von Taddicken und Schmidt (2017). Sozialen Medien bzw. Social Media bezeichnet demnach einen «Sammelbegriff für Angebote auf Grundlage[n] digital vernetzter Technologien, die es Menschen ermöglichen, Informationen aller Art zugänglich zu machen und davon ausgehend soziale Beziehungen zu knüpfen und/oder zu pflegen»

96 Exemplarisch für den deutschsprachigen sozial- und bildungswissenschaftlichen Diskurs kann an dieser Stelle auf die Begriffsdiskussion des Social Web (z. B. Schmidt 2008), sozialer Medien (z. B. Taddicken und Schmidt 2017) sowie des Web 2.0 verwiesen werden (z. B. Grell und Rau 2011; Reinmann 2010).

97 Ein erschöpfendes und trennscharfes Kategoriensystem ist gleichwohl nicht zu erwarten. 
(Taddicken und Schmidt 2017, 8). Die Wahl des Terminus soziale Medien bzw. Social Media zur Darstellung der folgenden Überlegungen begründet sich vor allem durch deren aktuelle Verbreitung. Wie Taddicken und Schmidt (2017, 9) am Beispiel einer Google-Suche demonstrieren, ist der Begriff «Social Media» wesentlich verbreiteter als der Begriff «Web 2.0». Die Orientierung am Begriffsverständnis von Taddicken und Schmidt (2017) begründet sich zum einen durch die Aktualität des erschienenen Handbuchs (Schmidt und Taddicken 2017). Zum anderen liegt die von Taddicken und Schmidt (2017) vertretende sozialwissenschaftliche Perspektive auf soziale Medien wesentlich näher an einer bildungswissenschaftlichen Position als beispielsweise eine informatische Sichtweise.

Relevant ist ebenfalls die Erkenntnis, dass die genannten Begriffe zwar in bildungswissenschaftliche bzw. medien- und hochschuldidaktische Diskussionen aufgenommen wurden, der Entstehungskontext jedoch kein genuin-pädagogischer ist (Grell und Rau 2011, 1 f.). Ferner wird in Betrachtung der jeweiligen Begriffsverwendungen sichtbar, dass es entsprechenden Termini in Bildungskontexten mitunter an einer präzisen Explikation mangelt. Im Diskurs über die Hochschullehre wird dies dann problematisch, wenn diese Begriffe als vermeintlich alles erklärende Labels verwendet werden und damit unrealistische Erwartungen über die Medienverwendung zukünftiger Studierender vermitteln. Zudem dürfen Termini wie soziale Medien, Web 2.0 oder Social Media nicht als Konzepte, die Aussagen über das konkrete Handeln von Menschen erlauben, missverstanden werden (Grell und Rau 2011, 3) Die vielfältigen Möglichkeiten zur aktiven Teilhabe und Mitgestaltung des Internets sind nicht zu verwechseln mit (impliziten) Annahmen über eine neue oder aktivere Teilhabe der jeweiligen Menschen. Gleichwohl lässt sich in Anlehnung an Jörissen und Marotzki (2007) eine mit dem Web 2.0 verbundene Vermassung technisch niedrigschwelliger Webanwendungen aufzeigen, welche eine aktive und produktive Teilhabe im World Wide Web ermöglichen (nicht aber quasi-automatisch herstellen).

Die Möglichkeit, Beiträge in themenspezifischen Gruppen von grossen OnlineNetzwerken (z. B. Facebook, Google+) zu erstellen, zu lesen und zu kommentieren sowie das gemeinsame Erarbeiten von Texten (z. B. via Etherpad, Wikipedia) sind nur wenige Beispiele für eine aktive und produktive Teilhabe durch sozialen Medien. Zugleich lassen diese Beispiele in Ansätzen erkennen, welche Bandbreite an digital vernetzter Technologien der Begriff soziale Medien umfasst. Um deutlicher zum Ausdruck zu bringen, welche spezifischen Merkmale von sozialen Medien im Folgenden im Fokus stehen werden, lohnt es sich, zwischen unterschiedlichen Formen oder Typen von sozialer Medien zu unterscheiden. Wie Taddicken und Schmidt $(2017,9)$ markieren, haben entsprechende Systematisierungen jedoch zwei Limitationen: So erscheint es Taddicken und Schmidt $(2017,9)$ kaum möglich, Gattungen systematisch hinsichtlich ihrer kommunikativen Prinzipien zu unterscheiden, da andere Anwendungen und Dienste diese aufgreifen können: «Facebook ist beispielsweise eine 
Netzwerkplattform, die auch Chatfunktionen anbietet; mixxt.de vereint Funktionalitäten von Diskussionsforen und Wikis; [...] etc.». Die zweite Grenze Systematisierung sehen Taddicken und Schmidt $(2017,9)$ in der hohen «Innovationsgeschwindigkeit im Bereich der sozialen Medien». So birgt jeder Versuch einer systematischen Differenzierung sozialer Medien die Gefahr, «nach gewisser Zeit veraltet und unvollständig zu sein» (Taddicken und Schmidt 2017, 9).

In Adaption und Vereinfachung bestehender Systematisierungen (z. B. Taddicken und Schmidt 2017; Grosseck 2009) wird im Folgenden zwischen drei unterschiedlichen Typen von sozialen Medien unterschieden. Als Unterscheidungskriterien dienen (a) der erkennbare Fokus der Anwendungen, (b) die zentralen Vernetzungsmodi zwischen Nutzerinnen und Nutzer sowie (c) mögliche Interaktionsformate sowie deren Grad an öffentlicher Zugänglichkeit. Unterschieden werden zwischen soziale Medien als

- Webanwendungen und Apps zur Knüpfung und Pflege von Kontakten (Kap. 4.3.3.1)

- Hochschulinterne Lernplattformen (Kap. 4.3.3.2)

- Sharing-Gemeinschaften auf Basis gemeinsamer Interessen (Kap. 4.3.3.3)

\subsubsection{Webanwendungen und Apps zur Knüpfung und Pflege von Kontakten}

Viele so genannter sozialer Medien werden als Webanwendung zur Knüpfung und Pflege von (privaten und/oder beruflichen) Kontakten charakterisiert und unter dem Begriff der sozialen Online-Netzwerke zusammengefasst. Diese sind vor allem für das Auffinden von Personen und Kontakten sowie für das Pflegen von (privaten und beruflichen) Beziehungen ausgelegt. Bekannte Beispiele hierfür sind Facebook ${ }^{98}$, XING ${ }^{99}$ oder LinkedIn ${ }^{100}$. Der charakteristische Fokus entsprechender Webanwendungen wird bei den angeführten Beispielen u. a. mit Blick auf die Landingpage erkennbar. Die Plattform XING «begrüsst» nicht registrierte Nutzerinnen und Nutzer beispielsweise zunächst mit der (nicht belegten) Faktenaussage: «50 \% aller Jobs werden über Kontakte vergeben» (Abb. 4.4), um darauf aufbauend den Fokus ihrer Webanwendung zum Ausdruck zu bringen: «Und XING ist das Netzwerk für berufliche Kontakte: 200.000 neue Kontakte werden hier jeden Tag geknüpft». Das Knüpfen von beruflichen Kontakten kann gemäss dieser Aussage als erkennbarer Fokus der Anwendung markiert werden. Auch auf der Landingpage von Facebook für nicht registrierte Nutzerinnen und Nutzer lässt sich eine ähnliche Formulierung finden: «Auf Facebook bleibst du mit Menschen in Verbindung und teilst Fotos, Videos und vieles mehr mit ihnen.» Mit Menschen im Verbindung zu blieben, wird von Facebook als erkennbarer Fokus markiert. Als charakteristisches Merkmal entsprechender

\footnotetext{
98 http://facebook.com (4.1.2017)

99 http://xing.com (4.1.2017)

100 http://www.linkedin.com (4.1.2017)
} 
Webanwendungen markieren Jörissen und Marotzki $(2009,199)$ zum einen, dass es strukturell die Möglichkeit gibt, sich zu «befreunden» bzw. zu vernetzen. Zum anderen wird in den Standardeinstellungen das Prinzip der «Freundesfreunde» genutzt, d.h. die jeweiligen Inhalte und Interaktionen können auch den Kontakten zweiten Grades zugänglich gemacht werden. Ferner findet sich in unterschiedlichen Variationen die Möglichkeit, die Kontakte anderer Personen einzusehen bzw. neue Kontakte werden auf Basis bereits bekannter Verbindungen vorgeschlagen.

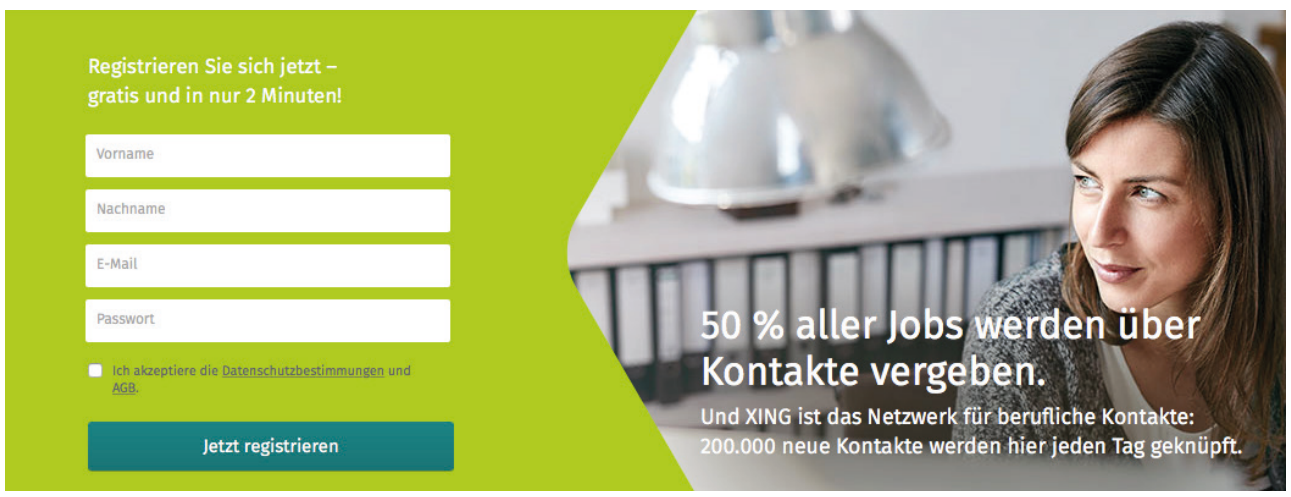

Abb. 4.4: Screenshot der Startseite des sozialen Netzwerkes XING.com (online abgerufen am 26. Februar 2016).

Diese Webanwendungen ermöglichen das Versenden von Nachrichten an konkrete Empfänger (ähnlich wie E-Mails oder SMS). Ferner eröffnen sie Anwendungen verschiedene Möglichkeiten, Mitteilungen oder Erfahrungen für alle Kontakte sichtbar (oder nur für bestimmte Teilgruppen zugänglich) zu teilen. Darunter fällt das Veröffentlichen von Fotos, das Posten von Videos, das Kommentieren von Nachrichtenbeiträgen oder das Schreiben einer Textnachricht. Aus dieser Vielzahl von persönlichen und professionell recherchierten Nachrichten von verschiedenen Akteuren entsteht ein ununterbrochener Strom zirkulierender Nachrichten welche wiederum kommentiert, "ge-liked» oder verschlagwortet werden können. In welchen Anteilen diese Netzwerke aktiv oder rezipierend genutzt werden, kann jede nutzende Person selbst entscheiden. Ebenso bleibt die Einschätzung der (sich teils widersprechenden) Informationen jeder Person selbst überlassen und stellt eine nicht unwesentliche Herausforderung im medienerzieherischen Sinne dar ${ }^{101}$.

Ferner bieten die Netzwerke die Möglichkeit, themenspezifischen Gruppen beizutreten, die mitunter auf Basis der Kontakte und der im Profil angegebenen Informationen vom Netzwerk empfohlen werden. Entsprechende Gruppen bieten in dieser Perspektive die Möglichkeit, die eigenen Kontakte zu erweitern. Zugleich eröffnen

101 Gleichwohl lässt sich spätestens seit der Wahl von Donald Trump eine öffentliche Debatte über das Erkennen und regulieren von (Fake-)Nachrichten erkennen: http://www.faz.net/aktuell/feuilleton/debatten/ hacker-kongress-ccc-widmet-sich-dem-thema-fake-news-14594184.html (29.12.2016). 
entsprechend spezifische Gruppen auch die Auseinandersetzung zum Austausch über ein spezifisches Thema - zunächst unabhängig davon, ob ein persönlicher Kontakt entsteht, welcher über das gemeinsame thematische Interesse hinausgeht. In diesem Sinne werden mit der Möglichkeit entsprechender Gruppen auch Prinzipien anderer Anwendungen (z. B. Diskussionsforen) adaptiert, wie von Taddicken und Schmidt $(2017,9)$ markiert wird.

\subsubsection{Hochschulinterne Lernplattformen}

Anders verhält es sich mit sozialen Medien im Kontext von (Hoch-)Schulen und Universitäten. Hier werden auch Lernplattformen bzw. Learning-Management-Systeme (oder spezifische Anwendungen entsprechender Systeme) als digitale soziale Medien verstanden. Bekannte Systeme wie z. B. Moodle ${ }^{102}$, Ilias ${ }^{103}$ oder Mahara ${ }^{104}$ bieten ebenfalls die Möglichkeit, Gruppen mit bestimmten Personen zu bilden. Sie dienen aber, anders als die zuvor beschriebenen Webanwendungen, nicht primär zur Knüpfung und Pflege von Kontakten zwischen Studierenden und Lehrenden. Vielmehr dienen entsprechende Systeme zur Unterstützung von Präsenzveranstaltungen oder zur Durchführung von Blended-Learning- oder Online-Kursen. Dieser Fokus entsprechender Lernplattformen und Learning-Management-Systeme kann am Beispiel der Landingpage der Moodleinstanz der TU Darmstadt illustriert werden (Abb. 4.5). So geht es nicht primär darum, sich untereinander zu vernetzen, sondern für Studierende steht das Einschreiben in Kurse im Mittelpunkt und für Lehrende die Frage, wie Kurse erstellt werden können.

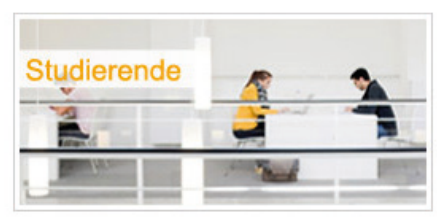

Ich komme nicht in einen Kurs...

...aus dem vergangenen Semester. ....aus dem aktuellen Semester.

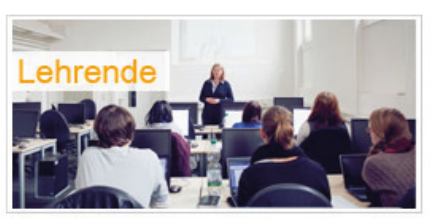

Wie bekomme ich einen Kurs...

...der mit TUCaN synchronisiert ist und in den alle Teilnehmer automatisch übertragen werden?

...ohne Synchronisation?

Abb. 4.5: Screenshot der Startseite von Moodle an der TU Darmstadt (online abgerufen am 26. Februar 2016).

Eine Vernetzung und Zusammenarbeit der Personen erfolgt im Rahmen konkreter Lehrveranstaltungen und gestellter Aufgaben. Häufig endet der Austausch zwischen den beteiligten Personen mit dem Ende der jeweiligen Lehrveranstaltungen.

102 https://moodle.de/ (25.01.2019).

103 https://www.ilias.de/ (25.01.2019).

104 https://mahara.org/ (25.01.2019). 
Eine Vernetzung der beteiligten Personen über Lehrveranstaltungen hinweg steht nicht im Fokus von Systemen wie Moodle oder Ilias. Im Gegensatz zu der vorherigen Kategorie von sozialen Medien basiert die Nutzung dieser sozialen Medien für Lernende mitunter auf Basis obligatorischer Lehrveranstaltungsanforderungen. Ebenfalls häufig gerahmt ist die Entscheidung, welche Lernplattformen und Learning-Management-Systeme an der jeweiligen Institution verwendet werden.

Bei Betrachtung möglicher Interaktionsformate lassen sich Ähnlichkeiten markieren: Lernplattformen und Learning-Management-Systeme beinhalten in der Regel ebenfalls die Möglichkeit, Direktnachrichten an konkrete Personen zu verschicken. Zudem bieten Plattformen wie Moodle die Möglichkeit, eigene Blogs zu führen. Wie mit Hinweis auf Taddicken und Schmidt $(2017,9)$ bereits markiert wurde, stellt sich für eine Differenzierung an dieser Stelle das Problem, dass Lernplattformen kommunikative Prinzipien anderer sozialer Medien adaptieren und ebenfalls verwenden. Im Mittelpunkt stehen allerdings lehrveranstaltungsbezogene Interaktionen in digitalen Kursräumen, zum Beispiel das (gemeinsame) Bearbeiten einer Aufgabe, das Lösen eines Quiz, das (Ver-)Teilen von Arbeitsmaterialien oder die Erstellung eines kollaborativen Textes im Rahmen eines Kurswikis. Ein Kurswiki als Teil einer übergeordneten Lernplattform basiert ähnlich wie plattforminterne Blogs auf ähnlichen oder gleichen kommunikativen Prinzipien. In der vorgeschlagenen Differenzierung sind diese jedoch von öffentlichen Sharing-Gemeinschaften wie z. B. Wikiia-Fan-Wikis ${ }^{105}$ oder Wikipedia ${ }^{106}$ dahingehend abzugrenzen, da sich der Kontext bzw. der erkennbare Fokus der Anwendungen unterscheidet.

Im Gegensatz zu Webanwendungen zum Pflegen von Kontakten (Kap. 4.3.3.1) oder zu Sharing-Gemeinschaften auf Basis gemeinsamer Interessen - wie zu zeigen sein wird (Kap. 4.3.3.3) - ist die öffentliche Zugänglichkeit der jeweiligen Interaktionen in den Standardeinstellungen von Lernplattformen häufig auf die jeweilige Lehrveranstaltung beschränkt. Eine Konfrontation mit einer Vielzahl unterschiedlicher Informationen und Einschätzungen bleibt - wenn nicht vom Lehrenden intendiert - in der Regel aus. In welchen Anteilen diese sozialen Medien von den Lernenden aktiv oder eher rezipierend genutzt werden, wird neben den medialen Möglichkeiten durch das didaktische Arrangement bzw. die Anforderungen der Lehrenden gerahmt.

105 https://www.wikia.com/explore (25.01.2019).

106 https://de.wikipedia.org/ (25.01.2019). 


\subsubsection{Sharing-Gemeinschaften auf Basis gemeinsamer Interessen}

Als dritte Kategorie von sozialen Medien werden Webanwendungen mit einem spezifischen (thematischen) Fokus und einer zugehörigen Community gefasst. Als typische Beispiele kann u. a. die Fotocommunity flickr ${ }^{107}$, Wikipedia ${ }^{108}$ als kooperativ erstellte Onlineenzyklopädie sowie die Ratgebercommunity gutefrage ${ }^{109}$ genannt werden. Diese sozialen Medien zeichnen sich dadurch aus, dass das gezielte Teilen von Wissen, Inhalten oder digitalen Artefakten im Mittelpunkt steht. Formen der Vernetzungen erfolgen in diesem Kontext durch die Arbeit an einer bestimmten Sache bzw. an einem gemeinsamen Thema, wie z. B. einem Wikipedia-Artikel. Dabei steht die Arbeit an einer gemeinsamen «Sache» im Fokus, weniger die Vernetzung untereinander. Exemplarisch illustrieren lässt sich dieser Fokus mit Blick auf die Landingpage der Flickr-Community (Abb. 4.6). Hier geht es darum, Teil einer Gemeinschaft zu werden, die sich durch das Interesse an Fotos auszeichnet. In ähnlicher Weise wenngleich weniger instruktiv - findet sich bei der Registrierung bei Wikipedia oder Wikibooks die Formulierung: «Wikipedia wird von Menschen wie dir geschaffen» bzw. «Wikibooks wird von Menschen wie dir geschaffen». Statt einer individuellen Vernetzung oder Verknüpfung steht in entsprechenden das gemeinsame Produkt bzw. gemeinsame «Sache» im Fokus.

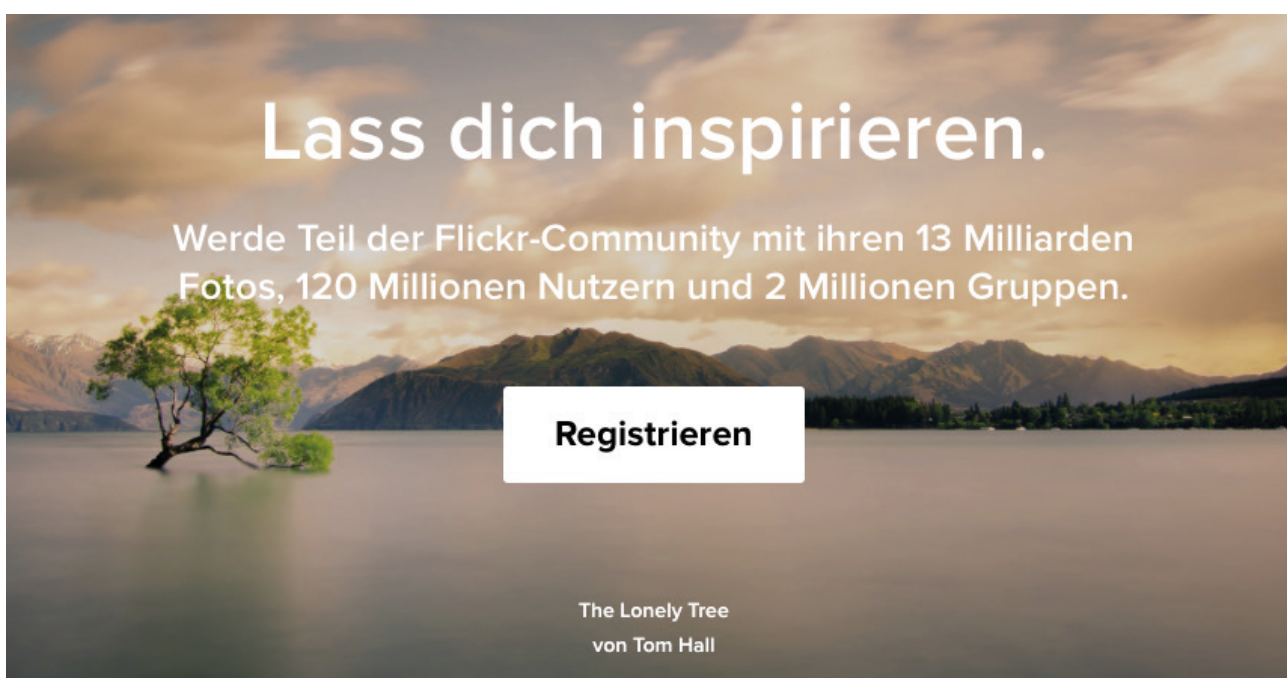

Abb. 4.6: Screenshot der Startseite der Fotocommunity flickr.com (online abgerufen am 26. Februar 2016).

Die Interaktionsmöglichkeiten der verschiedenen Plattformen fallen mitunter sehr unterschiedlich aus. Wikis eröffnen beispielsweise durch die Versionsgeschichte einen Einblick darin, wer wann welche Formulierungen ergänzt, gelöscht oder

107 https://www.flickr.com/ (25.01.2019).

108 https://de.wikipedia.org/ (25.01.2019).

109 https://www.gutefrage.net/ (25.01.2019). 
editiert hat. Die Berechtigung und Begründung entsprechender Änderungen werden öffentlich auf der Diskussionsseite thematisiert und ausgehandelt. Auf anderen Plattformen erfolgt die Vernetzung über die Vergabe von Tags und Schlagworten i.S.v. einer semantischen Indizierung - von digitalen Produkten (Jörissen und Marotzki 2007, 214). Durch die Verschlagwortung von Fotos (z. B. flickr $\left.{ }^{110}\right)$, Lesezeichen (z. B. bibsonomy ${ }^{111}$ ) oder Folien und Texten (z. B. slideshare ${ }^{112}$ ) werden beispielsweise Menschen sichtbar, die ähnliche Tags vergeben. Zugleich besteht auch bei entsprechenden Sharing-Plattformen - ähnlich wie bei Wikipedia oder der Ratgebercommunity gutefrage.net - die Möglichkeit verschiedene Artefakte zu kommentieren und zu diskutieren.

Die Frage der öffentlichen Zugänglichkeit ist im Vergleich zu geschlossenen Lernplattformen weitaus weniger restriktiv. Während Sharing-Gemeinschaften wie academia.eu ${ }^{113}$ oder researchgate ${ }^{114}$ als Webcommunities für Wissenschaftlerinnen und Wissenschaftlicher wissenschaftliche Artikel nur für Mitglieder der Community teilen, sind die Inhalte und digitalen Artefakte der Sharing-Gemeinschaften wie digitale Fotos auf flickr, Artikel auf Wikipedia oder Fragen und Antworten auf gutefrage.net, offen zugänglich. Für eine aktive Beteiligung bei entsprechenden Webcommunities ist gleichwohl ein Zugang notwendig. In welchen Anteilen diese Netzwerke aktiv oder rezipierend genutzt werden, kann selbst entschiedenen werden; häufig bleibt dieses auf eine kleine Gruppe von aktiven Mitgliedern beschränkt. Inwiefern ein Thema oder eine Frage dabei angemessen bzw. fachlich korrekt bearbeitet wird, ist jeweils abhängig von der Gemeinschaft bzw. von den Menschen, die sich beteiligen.

\subsubsection{Zwischenfazit}

Mit der Vorstellung der drei unterschiedlichen Typen von sozialen Medien wurde der Versuch unternommen, die weit gefasste Arbeitsdefinition von sozialen Medien nach Taddicken und Schmidt $(2017,8)$ zu präzisieren. Dabei konnten unterschiedliche Schwerpunktsetzungen identifiziert werden. So steht bei «Sharing-Gemeinschaften auf Basis gemeinsamer Interessen» in Anlehnung an die Arbeitsdefinition von Taddicken und Schmidt $(2017,8)$ vor allem die Möglichkeit im Fokus, «Informationen aller Art zugänglich zu machen». Der Schwerpunkt von «Webanwendungen zur Knüpfung und Pflege von Kontakten» richtet sich hingegen auf den zweiten Teil der Arbeitsdefinition von Taddicken und Schmidt $(2017,8)$ : «soziale Beziehungen zu knüpfen und/ oder zu pflegen». Die dritte Kategorie «hochschulinterne Lernplattformen» bietet

110 https://www.flickr.com/ (25.01.2019).

111 https://www.bibsonomy.org/ (25.01.2019).

112 https://de.slideshare.net/ (25.01.2019).

113 https://www.academia.edu/ (25.01.2019).

114 https://www.researchgate.net/ (25.01.2019). 
hinsichtlich der verschiedenen Vernetzungsmodi und Interaktionsformate verschiedene Gemeinsamkeiten zu den zwei vorherigen Differenzierungen. In Anknüpfung an Grosseck (2009) lässt sich die Gemeinsamkeit der drei Kategorien formulieren, dass entsprechende technischen Anwendungen nahezu intuitiv bedienbar sind und kaum informationstechnisches Verständnis voraussetzen. Allerdings sind diese technischen Möglichkeiten im Rahmen universitärer Bedingungen, wie z. B. Leistungsanforderungen deutlich anders kontextualisiert. Somit eröffnet die vorgeschlagene Differenzierung einen ersten begrifflichen Rahmen zur weiteren Diskussion lernförderlicher Potenziale sozialer Medien (Kap. 4.4.2) und deren spannungsvollen Verhältnissen zu universitären Rahmenbedingungen (Kap. 4.4.3).

\subsection{Sozialen Medien als Mittel zur Gestaltung von Hochschullehre}

Im Rahmen der Problematisierung der universitären Medienbildung im Lehramtsstudium wurde u. a. kritisiert, dass Studierende nur über begrenzte Möglichkeiten zum Lernen mit digitalen Medien verfügen (Kap. 4.2.2.1). Zugleich wird von ihnen als zukünftige Lehrkräfte erwartet, dass sie über eine medienpädagogische Kompetenz verfügen (Kap. 4.2.1). Ausgehend von der Annahme, dass eigene Lernerfahrungen mit digitalen Medien sinnvolle Anlässe eröffnen können, die eigene mediendidaktische Kompetenz zu entwickeln, widmet sich die vorliegende Arbeit im Folgenden einer mediendidaktisch geprägten Diskussion zur Gestaltung einer universitären Lehrerinnen- und Lehrerbildung mit sozialen Medien. Anknüpfend an Vorarbeiten (Rau 2017a, Rau 2017b) wird die universitäre Lehrerinnen- und Lehrerbildung zunächst in der Diskussion einer zeitgemässen Hochschullehre sowie im Zusammenhang der ELearning-2.0-Debatte verortet (Kap. 4.4.1). Daran anschliessend erfolgt ein Einblick in die theoretische Debatte zu Potenzialen sozialer Medien im Zusammenhang mit aktuellen Lernverständnissen (Kap. 4.4.2) sowie die exemplarische Diskussion empirischer Erkenntnisse zu aktuellen Spannungsfeldern in der Praxis (Kap. 4.4.3).

\subsubsection{Hochschuldidaktik und eLearning 2.0}

Eine zeitgemässe Betrachtung und Diskussion akademischer Lehre muss sich aus hochschuldidaktischer Perspektive am Leitbild des Wandels vom Lehren zum Lernen orientieren. Statt Inhalte und Lehrende in den Mittelpunkt didaktischer Überlegungen zu stellen, wird gemäss Wildt $(2013,40)$ eine Lehrauffassung angestrebt, die Lernprozesse und Studierende fokussiert. Statt tradierter rezeptionsorientierter Formate sollen vor allem Varianten des «aktiven und kooperativen bzw. kollaborativen Lernens» (ebd.) in der Lehre realisiert werden. In Anlehnung an konstruktivistische Ansätze wird Lernen in diesem Verständnis als ein aktiver (Konstruktions-) Prozess aufgefasst. Gefördert werden kann ein entsprechendes Lernen in formalen 
Bildungskontexten z. B. durch die Schaffung authentischer Problemsituationen sowie durch die Unterstützung kooperativer und kollaborativer Aktivitäten (z. B. Arnold und Kempkes 1998; Schulz-Zander und Tulodziecki 2011; Wildt 2013). Als Konsequenz dieses angestrebten Wandels von Lehr- und Lernkulturen kann mit Schneider et al. (2009) für die Hochschullehre festgehalten werden, dass Lehrende herausgefordert sind, «Lehre aus der Perspektive des Lernens neu zu durchdenken» (Schneider et al. 2009, 5). Mit der Integration digitaler Medien in der akademischen Lehre wird häufig die Hoffnung verbunden, entsprechende hochschuldidaktische Innovationen voranzutreiben und einen Wandel von Lehr- und Lernkulturen unterstützen zu können (Mayrberger 2007). In Anlehnung an diese Hoffnung zielen die folgenden Ausführen darauf ab, das Potenzial von sozialen Medien für ein aktives und kollaboratives Lernen in der Hochschullehre zu diskutieren. Diese Überlegungen markieren sowohl auf wissenschaftlicher als auch auf praxisbezogener Ebene einen zentralen Ausgangspunkt dieser Arbeit.

Die Veränderung von Lehr- und Lernkulturen in der deutschsprachigen Diskussion wurde zwischen 2007 und 2013 vor allem in Betrachtung neuer Praktiken mit dem Social Web diskutiert ${ }^{115}$. Beispielsweise formuliert Ehlers (2010) die These, dass sich mit der E-Learning-2.0-Debatte die Metapher für das Lernen geändert habe: «Von Rezeption zur Partizipation» (Ehlers 2010, 66). Mit mehr Enthusiasmus betont Wheeler (2009) das Potenzial vom Social Web für Bildungskontexte: «lt would be foolish to ignore the tremendous opportunities» (Wheeler 2009, 3). Neben der Betonung technologischer Potenziale zur Eröffnung neuer Lernpraktiken und den damit erhofften Veränderungen in institutionellen Kontexten, erscheint der Diskurs über digitale Medien in der Lehre aber auch geprägt von (vor-)schnellen Enttäuschungen (Gouseti 2010). Mit Blick auf das Social Web in Bildungskontexten konstatieren z. B. Köhler und Neumann $(2011,11)$ bereits im Jahr 2011, dass sich «in Bezug auf die Adoption entsprechender Praktiken eine gewisse Ernüchterung eingestellt [hat]». Entsprechend allgemein formulierte Aussagen müssen in Betrachtung fehlender systematischer Forschungssynthesen für den deutschsprachigen Diskurs jedoch skeptisch betrachtet werden (Rau 2017a).

Zur vertiefenden Auseinandersetzung mit den skizzierten Diskursen werden im folgenden Kapitel konkrete Erfahrungen und Erkenntnisse zum interaktiven und

115 Als Referenzpunkte dieser Zeitangabe dienten Beiträge und Tagungsbände der Gesellschaft für Medien in der Wissenschaft. Ohne Anspruch auf Vollständigkeit wurde der Beginn der Debatte über soziale Medien in der Hochschullehre mit der Erscheinung des Buches «Online-Communities als soziale Systeme: Wikis, Weblogs und Social Software im E-Learning» von Dittler (2007) auf das Jahr 2007 datiert. In den folgenden Jahren finden sich verschiedene Beiträge zum Einsatz von sozialen Medien in der Hochschullehre. Die Anzahl an Beiträgen geht, wie von Rau (2017a) gezeigt wird, im Jahr 2013 deutlich zurück. Daher wurde das Ende des «Hypes» auf das Jahr 2013 für diesen Zusammenhang datiert. Wie im Kapitel gezeigt wird, gibt es gleichwohl gute Gründe, die Diskussion über das Lehren und Lernen mit sozialen Medien fortzuführen. Zudem finden sich im internationalen Kontext weiterhin Publikationen zu sozialen Medien in Bildungskontexten wie exemplarisch am Themenheft «Social media and education ... now the dust has settled» von Selwyn und Stirling (2016) belegt werden kann. 
kollaborativen Lernen mit digitalen sozialen Medien skizziert. Im Fokus stehen die Fragen:

- Wie wurde akademische Lehre mithilfe digitaler sozialer Medien gestaltet?

- Welche spezifischen (neuen) Herausforderungen stellen sich in institutionellen Lehr- und Lernarrangements mit sozialen Medien?

Die Diskussion und Beantwortung dieser Fragen erfolgt in Betrachtung der Mikroebene von Hochschullehre. Zugleich werden Bezüge zu den universitären Rahmenbedingungen aufgezeigt. In Anknüpfung an das Leitbild des Wandels vom Lehren zum Lernen werden vor allem interaktive und kollaborative Praktiken mit sozialen Medien in der Hochschullehre betrachtet.

\subsubsection{Zwischen aktuellen Lernverständnissen und Potenzialen sozialer Medien}

Studierende in den Mittelpunkt der akademischen Lehre zu stellen und «Lehre aus der Perspektive des Lernens neu zu durchdenken» (Schneider et al. 2009, 5) wurde zu Beginn als hochschuldidaktisches Leitbild für eine zeitgemässe Hochschullehre skizziert. Die Integration digitaler Medien in die akademische Lehre, so lautet die Hoffnung, kann dazu führen, entsprechende hochschuldidaktische Innovationen voranzutreiben und einen Wandel von Lehr- und Lernkulturen zu unterstützen (Mayrberger 2007). In diesem Zusammenhang finden sich diverse Themenhefte von wissenschaftlichen Zeitschriften mit empirischen Erkenntnissen zum Lehren und Lernen mit sozialen Medien respektive dem Social Web respektive Web 2.0 (z. B. Berlanga et al. 2010; Mayrberger und Moser 2011; Meyer 2010; Selwyn und Stirling 2016) sowie erste konzeptionelle Überlegungen zu einer partizipativen Mediendidaktik (Mayrberger 2013a, 2014). Vor dem Hintergrund der vielfältigen Diskussionsbeiträge wird mit dem vorliegenden Kapitel nicht der Anspruch verfolgt, den kompletten Diskurs zu rekonstruieren. Vielmehr dient das Kapitel als erster Einblick in die mediendidaktische Diskussion über soziale Medien in der Hochschullehre. Das vorliegende Kapitel beschränkt sich dafür auf eine eher skizzenhafte Betrachtung der Diskussion von Lernen mit sozialen Medien bzw. auf die Diskussion der Frage, inwiefern das (gemeinsame) Erstellen und Teilen digitaler Produkte mit sozialen Medien das Lernen von Studierenden unterstützen und fördern kann. Dafür werden zwei Aspekte skizziert: (1) Neue Möglichkeiten zum aktiven und partizipativen Lernen sowie (2) Reflexionsanlässe und Praktiken des Sharings ${ }^{116}$.

116 Eine ausführlichere Darstellung des zugehörigen Diskurses findet sich bei Rau (2013). Gleichwohl erfolgte die Diskussion dieser Potenziale zu dieser Zeit unter dem Begriff Social Software. 


\subsubsection{Neue Möglichkeiten zum aktiven und partizipativen Lernen}

In der E-Learning-2.0-Debatte wird u. a. von Ehlers $(2010,66)$ die Perspektive vertreten, Lernende als Impulsgeber ihrer eigenen Lernprozesse zu verstehen und sie aktiv als (Ko-)Konstrukteure von Wissen und Materialien in Lehr- und Lernarrangements einzubinden. Pointierter formuliert: «Nicht die Rezeption, sondern die aktive Beteiligung steht im Vordergrund» (Ehlers 2010, 66). Die Möglichkeiten, als Lernende aktiv und selbsttätig (und nicht bloss Aufgaben erfüllend) in einer Lehrveranstaltung zu partizipieren, d. h. als Impulsgeberin bzw. Impulsgeber agieren zu können, werden unter anderem durch den Einsatz der verwendeten Medien gerahmt. Sozialen Medien wird in diesem Kontext das Potenzial zugesprochen, dass alle Beteiligten, insbesondere die Lernenden, neue Möglichkeiten erhalten, sich mitbestimmend mit ihren Erkenntnissen, Meinungen und Bewertungen in einen Gestaltungsprozess einbringen zu können (Mayrberger 2010a). So bieten beispielsweise Blogs die Möglichkeit, das eigene Denken - durch die Verschriftlichung eigener Ideen und Überlegungen - sichtbar und somit für Reflexionen und Rückmeldungen nutzbar zu machen (Luehmann 2008). Die Schaffung neuer Aktivitätsmöglichkeiten und Partizipationschancen als lernförderlich zu betrachten, begründet sich in Anlehnung an konstruktivistische Ansätze (Gerstenmaier und Mandl 1995). Um träges Wissen zu vermeiden, wird die Bedeutung von Eigenaktivität und Selbsttätigkeit als relevante Bedingung für Lernen markiert (Arnold und Kempkes 1998; Schulz-Zander und Tulodziecki 2011).

Ferner wird die Position vertreten, dass das Social Web nicht nur neue Möglichkeiten für Eigenaktivität und Partizipation eröffnet, sondern diese auch «aktiv» unterstützt. Argumentationen wie von Grosseck (2009) verweisen dabei u. a. auf die sinkenden technischen Hürden oder den Verbreitungsgrad von Social-Web-Anwendungen. Mcloughlin und Lee $(2007,667)$ vertreten ebenfalls die Position, dass das Web 2.0 bzw. das Social Web die Kreation bzw. Konstruktion von «Content» gegenüber dem blossen Konsum von Inhalten fördert. Eine ähnliche Position scheinen auch Iske und Marotzki (2010) mit Blick auf Wikis zu vertreten. So zielen Wikis nach Iske und Marotzki $(2010,149)$ "grundsätzlich auf die Transformation von Nutzern zu Autoren, von der Rezeption von Wissen zur Generierung von Wissen, von der individuellen Nutzung zur Nutzung in einer Gruppe»117.

In Betrachtung systematischer Reviews (Grell und Rau 2011; Sim und Hew 2010) zeigt sich jedoch, dass die empirische Realisierung dieser aktiven Unterstützung keinesfalls «automatisch» eintritt. Sowohl Sim und Hew (2010) als auch Grell und Rau (2011) machen in ihrer Analyse empirischer Beiträge zum Einsatz von (Micro-)Blogs und Wikis in der Hochschullehre deutlich, dass die aktive Beteiligung und Partizipation der Studierenden mithilfe sozialer Medien nur innerhalb bestimmter didaktischer Rahmungen gelingt.

117 Gleichwohl diskutieren Iske und Marotzki (2010) das Potenzial von Wikis nicht im Kontext institutioneller Bildungsinstitutionen. 


\subsubsection{Reflexionsanlässe und Praktiken des Sharings}

Anknüpfend an Iske und Marotzki (2010) kann die Ermöglichung von Praktiken des Sharings bzw. der verschiedenen Formen von Kollaboration und Kooperation innerhalb von (informellen) Lerngemeinschaften als weiteres lernförderliches Potenzial betrachtet werden. Mit dem Einsatz des Social Web und den damit verbundenen neuen Inhaltsformen können Ideen und Gedankengänge einzelner Personen dokumentierbar artikuliert und über soziale Medien geteilt werden, wodurch neue Möglichkeiten der eigenen und wechselseitigen Referenzierung entstehen. Möglichkeiten und Praktiken kollaborativen "Sharings» werden u. a. mit Bezug auf konkrete digitale Netzwerke wie Facebook thematisiert (Aydin 2012), aber auch im Kontext von persönlichen Gruppenblogs (Mcloughlin und Lee 2007) und Wikis (Iske und Marotzki 2010) diskutiert. Iske und Marotzki $(2010,146)$ weisen darauf hin, dass durch diese Artikulationen «Erfahrungen sowohl für den Autor wie auch für Dritte überhaupt erst sichtbar, adressierbar und referenzierbar und damit zum Ausgangspunkt individueller wie kollaborativer Nutzung ('sharing)) [werden]». Kollaboration erfolgt im Kontext inhaltlicher Auseinandersetzung und basiert auf dem Teilen von Informationen, Artefakten, Erfahrungen und Erkenntnissen. Sowohl Guth und Petrucco (2009) als auch Owen et al. (2006) weisen darauf hin, dass dieser Prozess Ausgangspunkt von Lern- und Transformationsprozessen sein kann: «The process of sharing knowledge often involves the process of transforming tacit knowledge into explicit knowledge, which is where true learning takes place» (Guth und Petrucco 2009, 426). Zudem wird die Bedeutung von kooperativen und kollaborativen Aktivitäten im Kontext konstruktivistischer Lerntheorien markiert (Schulz-Zander und Tulodziecki 2011).

Darüber hinaus wird von Owen et al. $(2006,25)$ vor allem das Auffinden von Menschen mit gleichen (oder komplementären) Interessen als zentrales Potenzial vom Social Web betont. Baumgartner (2006) veranschaulicht wie mithilfe von «Social Bookmarking»-Tools neue Recherchemethoden, die in Hochschulkursen Verwendung finden können. Über gleiche Internet- und Literaturreferenzen wird damit ermöglicht, mit Personen mit ähnlichen Interessen bzw. einschlägigen Expertinnen und Experten in Kontakt zu kommen (Owen et al. 2006). Die Möglichkeiten, interessante Menschen zu finden und mit ihnen kommunizieren zu können, sind auch für Aydin (2012) gute Gründe, warum auch digitale soziale Netzwerke wie Facebook in der institutionellen Lehre eingesetzt werden sollten. Guth und Petrucco (2009) betonen darüber hinaus die Möglichkeiten, in Lehr- und Lernarrangements über den eigenen Tellerrand zu schauen: «[...] social software tools give students the opportunity to interact with experts and novices alike on a global scale beyond planned classroom activities» (Guth und Petrucco 2009, 426). Für Owen et al. (2006) können so Grenzen heterogener Lerngruppen aufgelöst werden, denn in Online-Communities erzeugen Unterschiede bezüglich des Alters, Vorwissens, Geschlechts oder der Ortsgebundenheit keine Barrieren mehr (Owen et al. 2006, 45). 
Bei Betrachtung der Argumentationen wird zum einen sichtbar, dass die verschiedenen Autorinnen soziale Medien vor allem im Sinne von Sharing-Gemeinschaften auf Basis gemeinsamer Interessen verstehen. Zum anderen fokussieren die verschiedenen Argumente vor allem neue technische Möglichkeiten, welche in dieser Form zuvor nicht existierten. Vor diesem Hintergrund lässt sich als Zwischenfazit zunächst markieren, dass in Anlehnung an die skizzierten Argumente im Folgenden davon ausgegangen wird, dass mit sozialen Medien - vor allem aber nicht ausschliesslich im Sinne von Sharing-Gemeinschaften auf Basis gemeinsamer Interessen - verschiedene Potenziale einhergehen, Lern- und Bildungsprozess begleitende, kommunikative und kooperative Prozesse zu befördern. Diese Annahme muss jedoch dahingehend eingeschränkt werden, dass die Frage, inwiefern Lernende daran interessiert sind, diese Potenziale wahrzunehmen - z. B. den geschützten Raum der jeweiligen Lehrveranstaltungen überhaupt zu verlassen - zunächst offenbleibt. Ebenso stellt sich die Frage, inwiefern die universitären Rahmenbedingungen die skizzierten Möglichkeiten begrenzen oder gegebenenfalls verhindern.

\subsubsection{Erkenntnisse über Spannungsfelder in der Praxis}

Beim Versuch, die skizzierten Potenziale sozialer Medien unter universitären Rahmenbedingungen zur Entfaltung zu bringen, offenbaren sich spätestens bei der Durchführung konkreter Veranstaltungen verschiedene Herausforderungen. Mit Bezug auf die markierten Fragen werden zur Veranschaulichung dieser Herausforderungen drei ausgewählte Spannungsfelder diskutiert ${ }^{118}$. Diese Spannungsfelder bewegen sich (a) zwischen kollaborativen Lernformaten und individueller Leistungsbewertung, (b) zwischen (teil-)öffentlicher Sichtbarkeit und dem «Schonraum» akademischer Lehre sowie (c) zwischen einer individuellen Feedbackkultur und Massenveranstaltungen.

\subsubsection{Kollaborative Lernformate vs. individuelle Leistungsbewertung}

Die schriftliche Darstellung eigener Ideen und Überlegungen mithilfe von (Community-)Blogs, das Einüben des kooperativen Schreibens in öffentlichen Wikibooks oder die kooperative Form der Recherche mit Social-Bookmarking-Anwendungen sind Beispiele neuer kollaborativer Lernformate mit sozialen Medien. Diese Formate erscheinen passgenau zu zeitgemässen didaktischen Prinzipien, die «eigenaktiv-konstruierendes und kooperatives Lernen» betonen (Schulz-Zander und Tulodziecki 2011,

118 Die Darstellung der benannten Spannungsfelder erfolgt wie bei Rau (2017b) im Stil eines narrativen Reviews bzw. im Stil dichter Beschreibungen konkreter Fälle. Dafür wird auf ausgewählte Fallstudien zurückgegriffen (z. B. Ehlers et al. 2009). Zudem werden Beispiele aus eigenen (fach-)didaktischen Lehr-Lernprojekten mit Wikis an der Universität Potsdam (Rau et al. 2013) zur Veranschaulichung herangezogen. Im Fokus stehen dabei Blended-Learning-Konzepte, in denen der Einsatz von (kollaborativen) Online-Lernaktivitäten mit Aktivitäten der Präsenzveranstaltungen verzahnt wurden. 
42). Der Einsatz digitaler sozialer Medien in der Hochschule findet gleichwohl unter speziellen Rahmenbedingungen statt, die sich deutlich von den Rahmenbedingungen informeller Communities unterscheiden (Kap. 4.3.3.3). In einer Hochschulkultur, die einer individuellen Aneignungslogik folgt, und deren Anerkennungssysteme auf individuell identifizierbare Leistungen und Leistungsbewertung ausgerichtet sind, sorgen kollaborative Lernformate für spannungsvolle Verhältnisse (Dohn 2009). So müssen sich Lehrende z. B. mit der Frage auseinandersetzen, wie ein kollaborativ entstandener Wiki-Artikel von mehreren Studierenden zu bewerten ist. Die Fragen hierbei lauten: Werden kooperative und kollaborative Lehr- und Lernszenarios mit dem Ziel entwickelt, das Lernen aller beteiligten Studierenden zu unterstützen und sind dann Gruppenphänomene wie «Social Loafing» oder «Freeriding»" ${ }^{119}$ ein Problem? Bedarf es eines Mindestmasses an verpflichtender (und bewertungsrelevanter) Beteiligung für alle Gruppenmitglieder? Oder führt die Verpflichtung und Vorstrukturierung von Kooperationsprozessen gerade dazu, dass echte Kooperation und Kollaboration verhindert wird? Schliesslich könnten Studierende den Versuch unternehmen, die angeordneten kollaborativen Formen so zu bewältigen, dass diese den Vorstellungen der jeweiligen Lehrenden entspricht.

Innerhalb dieses Spannungsfeldes - zwischen anzuregenden kollaborativen Lernformaten einerseits und individuell zu bewertenden Leistungen anderseits sind Lehrende herausgefordert, eigene didaktische Gestaltungsentscheidungen zu treffen. Eine Möglichkeit zum Umgang mit dieser Herausforderung skizzieren Ehlers et al. (2009) in ihrer Fallstudie zum Einsatz von Blogs zur Unterstützung einer kompetenzförderlichen Lernumgebung. Im Rahmen der Lehrveranstaltung «Projektmanagement» (Studiengang BA «Wirtschaftsinformatik») sollte zur Schaffung einer entsprechenden Lernumgebung «das aktive Mitarbeiten und das Reflektieren durch die Studierenden» (Ehlers et al. 2009, 21) gefördert werden. In einem mehrphasigen Veranstaltungskonzept wurde das begleitende Schreiben in Blogs von Ehlers et al. $(2009,20)$ mit dem Ziel eingesetzt, "sowohl individuelle als auch Gruppen- und Peer-Reflexionsprozesse» zu unterstützen. Dafür erhielten Studierende die Aufgabe, einmal wöchentlich einen Blogbeitrag auf Basis von Orientierungs- und Reflexionsfragen zu schreiben. Im Rahmen der Veranstaltung arbeiteten die Studierenden in Projektgruppen zusammen. Ihre Blogbeiträge waren dabei nur innerhalb ihrer Gruppe einsehbar. Um Peer-Reviews zu ermöglichen, erhielt zudem eine weitere Projektgruppe Zugriff auf die Blogbeiträge ihrer Kommilitoninnen und Kommilitonen. Für die Bewertung der Veranstaltung wurde eine schriftliche Ausarbeitung, eine Klausur sowie die verfassten Blogbeiträge begutachtet. In ihrer Fallstudie berichten Ehlers et al. (2009) von der Erfahrung, dass Reflexionsleistungen für viele Studierende nicht als relevante Aktivitäten in einer Lehrveranstaltung wahrgenommen werden. Daher,

119 Mit den Begriffen «Freeriding» und «Social Loafing» werden Phänomene in Gruppenarbeiten beschrieben, bei den sich Studierende nicht in gleicher Weise beteiligen (Hall und Buzwell 2013). 
so die Konsequenz von Ehlers et al. (2009, 28), «muss Reflexion und die Arbeit im Weblog ein integraler Bestandteil des Kurs-Curriculums werden, der auch in die Bewertung mit einfließt». Zugleich sehen sie die Problematik, eigenständige Reflexionsleistungen durch Bewertungsvorgaben (und Orientierungsfragen) vorzustrukturieren und markieren in Betrachtung ihrer Praxiserfahrung, "dass es Studierenden bei der Entwicklung eigener Reflexionspraxis hilft» (Ehlers et al. 2009, 28).

Eine ähnliche Strategie zum Umgang mit diesem Spannungsfeld skizziert Öner (2009) in ihrer Fallstudie zur Unterstützung kollaborativer Unterrichtsplanung. Mit dem Einsatz von Wikis ${ }^{120}$ verfolgte Öner (2009) zum einen das Ziel, die Zusammenarbeit der Studierenden zu ermöglichen und zu unterstützen, zum anderen wurde angestrebt, den Prozess von Gruppenarbeiten sowie die individuelle Beteiligung jedes Gruppenmitglieds zu beobachten und nachvollziehen zu können. Im Rahmen eines Blended-Learning-Konzeptes erhielten die Studierenden dafür die Aufgabe, zwei Unterrichtsplanungen in kooperativer Zusammenarbeit zu erstellen. Die Aufgabenstellung für Studierende bestand darin, alle Gespräche und weitere Beteiligungen - jenseits des digitalen Austausches - im Wiki zu dokumentieren. Dies sollte auch dann erfolgen, wenn Gruppenmitglieder «offline» an der Aufgabenstellung gearbeitet hatten. Im Gegensatz zu Ehlers et al. (2009) begründete Öner (2009) diese Aufgabenstellung (gegenüber ihren Studierenden) nicht als Schaffung zusätzlicher Reflexionsanlässe für das eigene Handeln. Es ging ihr um eine gerechte Bewertung jedes einzelnen Studierenden. Auf Basis der Erfahrungen und einer anschliessenden Evaluation kommt Öner $(2009,2)$ jedoch zu dem Schluss, dass jene Aufgabenstellung, die eine gerechte Bewertung ermöglichen sollte, von verschiedenen Studierenden als irritierend wahrgenommen wurde und mehrere Studierende problematisierten, dass der so entstandene Bewertungsdruck eine wirkliche Zusammenarbeit eher verhindere.

Die vorgestellten Beispiele können hinsichtlich des Spannungsfeldes zwischen kollaborativen Lernformaten und individueller Leistungsbewertung als exemplarisch für eine Vielzahl von Fallstudien und Projekterfahrungen betrachtet werden. So zeigt sich bei systematischen Forschungsreviews von Sim und Hew (2010) sowie Grell und Rau (2011), dass der Verzicht auf verpflichtende bzw. bewertungsrelevante Aufgabenstellungen häufig mit ausbleibender studentischer Beteiligung verbunden ist. Ferner scheinen von offenen Lehr- und Lernszenarios nur jene Studierende zu profitieren, die bereits Fähigkeiten in und Interesse an selbst gesteuertem und eigenaktivkonstruierendem Lernen haben. Zugleich zeigt sich aber auch, dass mit restriktiven Entscheidungen zur Vorstrukturierung des didaktischen Settings die partizipativen und kollaborativen Potenziale von digitalen sozialen Medien überformt werden können. Dies kann unter anderem dazu führen, dass Studierende im Modus «playing the

120 Wenngleich Öner (2009) den Begriff Wiki verwendet, weist sie auch darauf hin, dass die Webapplikation GoogleDocs verwendet wurde. Die Funktionalitäten sind zwar ähnlich, zugleich überrascht die Bezeichnung Wiki an dieser Stelle. 
game» lediglich versuchen, den Anforderungen der Lehrenden gerecht zu werden, wie es bei Ebner et al. $(2010,97)$ beschrieben wird. Entsprechende studentische Handlungsstrategien können aus einer subjektwissenschaftlichen Perspektive als «defensives Lernen» bezeichnet werden (z. B. Grell 2006, Faulstich 2008). Im Vordergrund steht dabei das Bewältigen einer Situation aus einer Abwehrhaltung heraus, während die Bearbeitung echter Lernproblematiken aussen vor bleibt.

Das spannungsvolle Verhältnis zwischen kollaborativen Lernformaten einerseits sowie der individuellen Leistungsbewertung andererseits lässt sich mit sozialen Medien offensichtlich nicht auflösen. Vielmehr kann es aus studentischer Perspektive durchaus widersprüchlich erscheinen, warum zur Vorbereitung auf eine wissensbezogene Multiple-Choice-Klausur beispielsweise kollaborative Texte in einem Wiki geschrieben werden müssen. Ob sich die eingangs skizzierten Potenziale von sozialen Medien in Lehr- und Lernszenarien entfalten können, wenn Studierende diese als widersprüchlich erleben, muss bezweifelt werden. Für die Planung digitaler Lehr- und Lernszenarios ist es daher lohnenswert, die von Schulz (2006) formulierte Erkenntnis zu berücksichtigen, dass sich die verwendeten Vermittlungsformen und die zum Einsatz kommenden Prüfungsformen wechselseitig bedingen.

\subsubsection{2 Öffentliche Sichtbarkeit vs. «Schonraum» akademischer Lehre}

Ein weiteres Spannungsfeld entsteht zwischen klassischen Leistungen im Vergleich zu neuen Artefakten im Social Web. Dabei stellt sich insbesondere die Frage, welche Bedeutung die Sichtbarkeit dieser Artefakte für das Lehr- und Lerngeschehen hat. Richten sich traditionelle universitäre Leistungen, wie etwa das Schreiben von Essays und Hausarbeiten, aber auch das Bearbeiten von Klausuren, in der Regel an die jeweils Lehrenden und damit an konkrete Adressatinnen und Adressaten, lässt sich eine Differenz zu Formaten mit sozialen Medien markieren. Die verschiedenen Möglichkeiten zur Erstellung und Veröffentlichung von digitalen Materialien mithilfe sozialer Medien haben die Konsequenz, dass die Arbeitsergebnisse von Studierenden für eine (Teil-)Öffentlichkeit sichtbar werden. Studierende sind gewissermassen genötigt, ihr Denken für mehr Menschen als nur für die Lehrenden sichtbar zu machen und so ihren individuellen «Schonraum» zu verlassen. Diese Sichtbarkeit kann auf eine kleine Gruppe weiterer Studierender der gleichen Lehrveranstaltung beschränkt bleiben, wie es in den Beispielen von Ehlers et al. (2009) und Öner (2009) skizziert wurde. Über öffentliche Plattformen wie Wikipedia oder Wikibooks können die von Studierenden erstellten Produkte aber auch über die Grenzen des universitären «Schonraumes» hinaus sichtbar werden (z. B. Bonk et al. 2009). Jenseits theoretischer Lernpotenziale, stellt sich für die Studierenden in entsprechenden Settings zudem häufig die Frage, wie mit den Arbeiten von anderen Studierenden umgegangen werden kann. Inwiefern ist es beispielsweise erlaubt, sich Anregungen von anderen 
Studierenden zu holen oder inwiefern dürfen Formulierungen und Überlegungen anderer Studierender kopiert werden? Studierende äussern gegenüber Lehrenden auch die Sorge um ihr eigenes geistiges Eigentum. In der Veröffentlichung ihrer Ideen sehen sie u. a. die Gefahr, dass andere Studierende diese stehlen könnten (Waycott et al. 2013). Wenn Studierende aber keinen Sinn im (teil-)öffentlichen Austausch mit anderen Studierenden sehen, sondern vielmehr um den Schutz ihrer eigenen Ideen besorgt sind, ist die Entfaltung lernförderlicher Potenziale hier fraglich.

In Abhängigkeit vom didaktischen Einsatz digitaler sozialer Medien stellen sich die vorherigen Fragen innerhalb dieses Spannungsfeldes in unterschiedlicher Weise. Eine Variante zum Umgang dem beschriebenen Spannungsfeld lässt sich am Beispiel eines Seminars des Arbeitsbereiches Allgemeine Pädagogik mit dem Schwerpunkt Medienpädagogik an der TU Darmstadt veranschaulichen, in dem ein öffentliches Wiki als Schreib- und Feedbackumgebung zum Einsatz kommt. In der Veranstaltung sind Studierende (BA Pädagogik) mit der Aufgabe konfrontiert, eine forschungsmethodische Kritik zu einer publizierten empirischen Studie zu formulieren. Neben der Vermittlung von Kenntnissen über empirische Sozialforschung sollen Studierende dadurch ihre Kritikfähigkeit im Hinblick auf die methodische Qualität empirischer Studien weiterentwickeln. Als Strukturierungshilfe zur Entwicklung einer entsprechenden Kritik durchlaufen die Studierenden einen dreistufigen Prozess: Im ersten Schritt ist nach der Hälfte des Semesters eine erste Entwurfsfassung zu formulieren. Im zweiten Schritt sind die Studierenden aufgefordert, die Entwürfe ihrer Kommilitoninnen und Kommilitonen zu lesen und anderen Gruppen kriteriengeleitet Rückmeldung zu geben. Auf Basis des Peer-Feedbacks sowie der Rückmeldungen im Rahmen der Präsenzveranstaltungen sind die Studierenden im dritten Schritt aufgefordert, ihre Entwurfsfassung bis zum Ende des Semesters zu überarbeiten. Die Bearbeitung der ersten zwei Schritte ist verpflichtend und Voraussetzung für die Bewertung der finalen Version der forschungsmethodischen Kritik. Eine Studie wird jeweils von vier bis fünf Tandems analysiert, sodass sich Studierende über die eigene Tandemarbeit hinaus untereinander austauschen können. Damit die Studierenden frühere forschungsmethodische Kritiken lesen sowie den aktuellen Fortschritt ihrer Kommilitoninnen und Kommilitonen einsehen und bei Interesse kommentieren können, ist das Wiki unabhängig von konkreten Veranstaltungen öffentlich zugänglich. Die genannten Fragen und Sorgen von Studierenden zu diesem Veranstaltungskonzept werden seit mehreren Semestern in unterschiedlicher Form sichtbar, obwohl eine FAQ-Sammlung zur Verfügung steht, und die Studierenden zu einer quellentransparenten Bezugnahme ermuntert werden. Die Umgangsweisen der Studierenden mit dieser offenen Form des Peer-Feedbacks und der Überarbeitung eigener Texte reichen von (a) Plagiatsversuchen über (b) das Ignorieren anderer Arbeiten zum Schutz vor einer möglichen Beeinflussung der eigenen Arbeit bis hin zu (c) einem produktiven Austausch untereinander. Zudem thematisieren Studierende, dass das Formulieren schriftlicher Feedbacks eine nicht zu unterschätzende Herausforderung sei. 
Im Gegensatz zu Konzepten, in denen das (öffentliche) Teilen von Ideen und Arbeitsergebnissen gewünscht und gefordert wird, lassen sich auch Strategien von Lehrenden skizzieren, die die Sichtbarkeit studentischer Produkte über soziale Medien beschränken. Auf Basis einer Interviewstudie mit Hochschullehrenden beschreiben Waycott et al. (2013) hierzu verschiedene Varianten. Zum Schutz studentischer Ergebnisse vor einem möglichen Ideendiebstahl betonten Lehrende beispielsweise die Kontrollmöglichkeiten sozialer Medien (Waycott et al. 2013). Ein Lehrender verwies in dieser Logik, so Waycott et al. (2013), auf die Nutzung von Software zur Plagiatserkennung. Eine andere Strategie, die von Waycott et al. (2013) berichtet wurde, ist die Veränderung der Zugriffsrechte in unterschiedlichen Arbeitsphasen. So konnten die Ergebnisse von anderen Studierenden erst gesehen werden, wenn sie selbst etwas erstellt hatten. Durch diesen Zugriff konnten die Studierenden jedoch keinen Einfluss mehr auf die Arbeiten anderer Studierender nehmen. Die Lehrenden versuchten damit, der Sorge der Studierenden gerecht zu werden und einem Ideendiebstahl vorzubeugen. Es stellt sich jedoch die Frage, warum die Arbeitsergebnisse überhaupt (semi-)öffentlich geteilt werden mussten, da die verwendete Strategie zum Schutz einen kollaborativen Austausch nicht fördern. Waycott et al. (2013) weisen in der Diskussion dieser Strategie auch darauf hin, dass der scheinbare Schutz individueller studentischer Arbeiten einer tradierten Bewertungspraxis folgt. Die Frage, mit welchem Ziel und in welchem didaktischen Rahmen die Lehrenden den Einsatz ihrer Strategie auswählten, wird von Waycott et al. (2013) leider nicht präzisiert.

Das sich eröffnende Spannungsfeld zwischen der öffentlichen Sichtbarkeit studentischer Arbeitsergebnisse und dem traditionellen "Schonraum» akademischer Lehre, schafft Herausforderungen für alle Beteiligten. So lassen sich bei Studierenden verschiedene Unsicherheiten im Umgang mit (öffentlich) sichtbaren Arbeitsergebnissen identifizieren. Für Lehrende sind Entscheidungen zur didaktischen Konzeption hinsichtlich der Sichtbarkeit studentischer Ergebnisse keinesfalls trivial. Dabei zeigt sich erneut, dass das Teilen langfristig sichtbarer und kollaborativ erstellter Arbeitsergebnisse sowie die Ermunterung zum gegenseitigen Austausch nur begrenzt mit einer individuellen (und kompetitiven) Bewertungslogik vereinbar ist. Wenn Studierende in Lehrveranstaltungen einerseits traditionell individuell bewertet werden, anderseits aber aufgefordert werden, ihre selbst entwickelten Ideen und Lösungsstrategien öffentlich mit einer Gemeinschaft zu teilen, ist durchaus nachvollziehbar, dass Studierenden die eigenen Beiträge nicht vor einer Prüfung mit anderen teilen wollen. Zugleich zeigt sich, dass das Schreiben im Rahmen einer Community, die auch über die jeweils spezifische Veranstaltung hinaus besteht, jenseits der Prüfungsbewältigung von Studierenden als sinnvoll erlebt werden kann ${ }^{121}$.

121 Wie Dalsgaard und Paulsen (2009) weiterführend zeigen, ist diese Sichtbarkeit bzw. Transparenz von studentischen Interaktionen insbesondere bei reiner Online-Lehre bzw. im Fernstudium zum Aufbau einer Lerngemeinschaft bzw. zum gegenseitigen Support hilfreich. 


\subsubsection{Individuelle Feedbackkultur vs. universitäre Massenveranstaltungen}

Im Kontext des Wandels vom Lehren zum Lernen wird verstärkt eine studierendenzentrierte (statt lehrendenzentrierte) Lehre gefordert. Damit gehen auch veränderte Erwartungen an die Tätigkeiten der Lehrenden einher. King (1993) beschreibt diese Veränderung mit der Metapher «from the sage on the stage to the guide on the side» (King 1993, 30). In kritischer Abgrenzung von der rein instruktionsorientierten Lehre wird z. B. die Rolle der Lehrenden als Lernberaterinnen und Lernberater diskutiert. Vor diesem Hintergrund ist es relevant zu fragen, inwiefern sich die durch soziale Medien neu entstandenen Handlungsoptionen im Rahmen der Hochschulstrukturen nutzen lassen und wie ein angemessener Umgang mit neuen digitalen Artefakten und Interaktionsräumen in Lehr- und Lernkontexten erfolgen kann. Dies beinhaltet auch den von Grell und Rau (2011) markierten Aspekt, «mehr Aufmerksamkeit für den produktiven Umgang mit der Sichtbarkeit des individuellen und kollektiven 〈Noch-nicht-Verstanden-Habens〉 [...] zu entwickeln» (Grell und Rau 2011, 18). So ist auch zu fragen, welche Ansprüche sich an Lehrende hinsichtlich des Umgangs mit Kollektivprozessen einer gesamten Seminargruppe sowie der individuellen Schritte von Lernenden richten. Ebenfalls stellt sich die Frage, welches Interesse Studierende an regelmässigen Rückmeldungen und mäeutischen Nachfragen haben, die ihnen gegebenenfalls aufzeigen, welche Themen sie bisher noch nicht verstanden haben.

Ein möglicher Umgang mit diesen Fragen lässt sich am Beispiel einer physikdidaktischen Lehrveranstaltung an der Universität Potsdam skizzieren (Rau et al. 2013). Mithilfe eines geschlossenen Kurs-Wikis wurde versucht, eine konstruktive Feedback- und Diskussionskultur hinsichtlich physikdidaktischer Fragen zu etablieren. In einem Blended-Learning-Ansatz planten die Studierenden den Einsatz von Experimenten unter fachdidaktischen Aspekten und führten in diesem Zusammenhang experimentelle Tätigkeiten und Microteachings durch. Dafür bearbeiteten die Studierenden im Rahmen von drei thematischen Einheiten über jeweils vier Wochen die gleichen Planungsaufgaben (z. B. zur Erarbeitung von Schülervorstellungen). Bewertungsrelevant war eine von den Studierenden ausgewählte Planung zu einer der drei thematischen Einheiten. Die Bearbeitung der Aufgaben erfolgte in Tandems und musste jeweils zwei Tage vor der Präsenzveranstaltung im Wiki veröffentlicht werden. So hatten die Lehrenden «just in time» die Möglichkeit, schriftliches Feedback auf den Diskussionsseiten des Wikis zu formulieren und übergreifende Schwierigkeiten zu Beginn jeder Präsenzveranstaltung zu diskutieren. Der Umgang der Studierenden mit den regelmässig erhaltenen Rückmeldungen erfolgte gleichwohl sehr unterschiedlich. Auf Basis einer Alltagsempirie wurde zwischen drei Gruppen von Studierenden differenziert: (a) den passiven Aussenstehenden, (b) den stillen Weiterdenkerinnen und Weiterdenkern sowie (c) den aktiven Diskussionspartnerinnen und -partner. Die Gruppe der passiven Aussenstehenden ist dadurch charakterisiert, dass sie die erhaltenen Rückmeldungen nicht bzw. erst im Rahmen der Veranstaltung 
zur Kenntnis nahmen. Die Gruppe der stillen Weiterdenkerinnen und Weiterdenkern setzte sich vor der Veranstaltung mit den Rückmeldungen auseinander und konnten in der Präsenzdiskussion neue Ideen und Problemlösungen einbringen. Die (kleine) Gruppe der aktiven Diskussionspartnerinnen und -partner äusserte sich bereits vor dem Präsenztermin online zu dem erhaltenen Feedback in Form von vertiefenden Nachfragen oder alternativen Vorschlägen. Während in den Diskussionen mit den "passiven Aussenstehenden» bei den Lehrenden der Eindruck entstand, die bereits online formulierten Rückmeldungen müssten in der Präsenz erneut formuliert werden, wurden die Diskussionen mit den zwei weiteren Gruppen sowohl online als auch in der Präsenz als sehr bereichernd erlebt. Zudem konnten die Lehrenden an den online formulierten Antworten der Studierenden erkennen, wie intensiv sich diese mit den jeweiligen Themen auseinandergesetzt hatten. Diese Transparenz ermöglichte es den Lehrenden auch, die während der Präsenz formulierten Aussagen von Studierenden (sowie deren Beteiligung bzw. Nichtbeteiligung), mit den online formulierten Antworten in Beziehung zu setzen und ihre Erkenntnisse zur thematischen Auseinandersetzung der Studierenden zu erweitern.

So anregend und bereichernd die Diskussionen mit den Studierenden einerseits wahrgenommen wurden, so herausfordernd erwies sich für die Lehrenden andererseits das Schreiben der Rückmeldungen und das Beantworten der Nachfragen. Insbesondere der Anspruch, individuelle Rückmeldungen jenseits von Textbausteinen zu formulieren, ist in Abhängigkeit der Anzahl der Teilnehmerinnen und Teilnehmer eine nicht zu unterschätzende zeitliche Herausforderung für Lehrende. Im Rahmen der zuvor skizzierten fachdidaktischen Lehrveranstaltung mit jeweils vier bis acht studentischen Beiträgen pro Woche zeigte sich, dass es für Lehrende kaum möglich war, auf sämtliche studentischen Rückfragen reagieren zu können. Wurden mehrere Veranstaltungen mit entsprechend intensiven Feedbackszenarien angeboten, konnten Rückmeldungen nur noch in schematischer und wenig individualisierter Form erfolgen. Vor diesem Hintergrund lässt sich mit Blick auf die eingangs formulierten Fragen festhalten, dass die Hoffnung, Lehrende könnten mithilfe digitaler sozialer Medien auf sämtliche studentische Ideen und Produkte reagieren und so «zum omnipräsenten Coach aller Bildungsprozesse» (Grell und Rau 2011, 19) werden, im Kontext von Seminaren mit bis zu 50 Personen, die es sie an verschiedenen deutschen Hochschulen regelmässig geben soll, kaum realistisch ist. Auch in diesem Spannungsfeld wird sichtbar, dass die Organisationslogik von formalen Bildungsinstitutionen deutlich andere Ziele verfolgt, als die von einer Lehr- und Lernkultur des «Wandels vom Lehren zum Lernen» angestrebten Ziele. 


\subsubsection{Zwischenfazit}

Die bisherigen Ausführungen zeigten Gestaltungsmöglichkeiten interaktiver und kollaborativer Hochschullehre und markierten spezifische Herausforderungen, die mit dem Einsatz digitaler sozialer Medien einhergehen. Dafür wurden exemplarische Szenarien mit Blogs als Reflexionsinstrumente sowie Wikis als Feedbackumgebungen skizziert. Wenngleich mit Blogs und Wikis eher typische - gemäss des öffentlichen Sprachgebrauchs - soziale Medien in den Beispielen verwendet wurden, lassen sich diese gemäss der vorgestellten Begriffsdiskussion vor allem als hochschulinterne Lernplattformen bzw. hochschulinterne Wikis und Blogs verstehen. Der Medieneinsatz war dabei jeweils in spezifische Blended-Learning-Konzepte eingebettet, in denen Präsenz- und Onlinephasen miteinander verzahnt wurden. Die mit der Verwendung von Blogs und Wikis angestrebten Ziele, z. B. Austausch- und Kollaborationsprozesse anzuregen, wurden jedoch mit unterschiedlichen Strategien verfolgt. Die erläuterten Spannungsfelder geben einen ersten Einblick, zwischen welchen spannungsgeladenen Entscheidungen Lehrende balancieren und unter welchen herausfordernden Bedingungen sie agieren müssen. Sichtbar werden diese Herausforderungen in Bewertungssituationen kollaborativer Lernformate, im Umgang mit neuen Inhalts- und Ausdrucksformen digitaler Produkte und dem Anspruch an eine Feedbackkultur im Kontext von Massenveranstaltungen.

In Anlehnung an Dohn (2009) lassen sich diese Herausforderungen grundsätzlich auf die Diskrepanz zwischen den skizzierten Lernverständnissen sowie der Organisationslogik formaler Bildungsinstitutionen zurückführen. Zum anderen lassen sich mit Waycott et al. (2013) sowie Grell und Rau (2011) spezifische Probleme markieren, die mit dem Einsatz digitaler sozialer Medien einhergehen. Mit Blick auf das skizzierte Leitbild des Wandels vom Lehren kann für die Praxis der Hochschullehre festgehalten werden, dass der Einsatz digitaler Medien - und das ist nicht überraschend - keine bereits vorhandenen Spannungsfelder auflösen kann. Eine angemessene Balance zwischen den anzustrebenden Lehraktivitäten und den rahmenden Hochschulstrukturen zu finden, bleibt eine jeweils kontextspezifische Herausforderung. Zugleich zeigt sich mit Blick auf die skizzierten Beispiele, dass unter der Anerkennung der gegebenen Rahmenbedingungen die sich eröffnenden Potenziale in Ansätzen genutzt werden können. Diese Erkenntnisse dienen als Orientierung für den zu formulierenden Entwurf (Kap. 6).

\subsection{Soziale Medien als Gegenstand universitärer Medienbildung}

In der bildungspolitischen und-administrativen Diskussion wird die Realisierung von Medienbildung in Bildungsinstitutionen in Form eines integrativen Ansatzes gefordert (Kap. 4.2.1.3). Das Lernen soll, so die Leitidee dieses Ansatzes, mit und über Medien erfolgen. Im Fokus des aktuellen Kapitels steht nun die Perspektive des Lernens 
über Medien. Diese Betrachtung des Zusammenhangs zwischen Lernen und Medien wird in der medienpädagogischen Fachdiskussion häufig als «Medienerziehung» bzw. als medienerzieherische Perspektive bezeichnet (z. B. Ruge 2014; Tulodziecki et al. 2010). Das Lehren und Lernen über Medien kann in diesem Zusammenhang auch als (schulische) Medienkompetenzförderung und -entwicklung verstanden werden. Um einen Einblick in die medienpädagogische Fachdebatte zu eröffnen und zentrale Ideen für die Entwicklung eines Entwurfs zu skizzieren, erfolgt im ersten Schritt eine Auseinandersetzung mit medienerzieherischen Überlegungen zum Begriff der Medienkompetenz sowie zum Konzept der handlungsorientierten Medienerziehung (Kap. 4.5.1). Daran anknüpfend erfolgt im zweiten Schritt eine Betrachtung aktueller Themen- und Fragestellungen, welche in der Auseinandersetzung mit sozialen Medien zentral sind (Kap. 4.5.2).

\subsubsection{Medienerzieherische Perspektive}

In der bildungspolitischen Diskussion (Kap. 4.2.1) wird die Förderung bzw. die Entwicklung von Medienkompetenz als Ziel eines Lernens über Medien formuliert. Der Begriff Medienkompetenz wurde in der vorgenommenen Begriffsklärung als Möglichkeit verstanden, medienpädagogische Zielstellungen von Lehraktivitäten (und Ergebnisse individueller Lernprozesse) zu beschreiben (Kap. 4.3.1.5). In einer ersten Arbeitsdefinition wurde Medienkompetenz konkretisiert als «Fähigkeit und Bereitschaft zu einem sachgerechten, selbstbestimmten, kreativen und sozial verantwortlichen Handeln in einer von Medien mitgestalteten Welt» (Tulodziecki 1997, 116). Ferner wurde der Begriff Medienkompetenz im Kontext einer Medienbildung für Lehrerinnen und Lehrer als Bestandteil einer medienpädagogischen Kompetenz diskutiert (Kap. 4.3.2.4). Anknüpfend an diese Überlegungen widmet sich das folgende Kapitel zwei Fragestellungen:

- Welche Aufgaben und Ziele werden aus medienpädagogischer Perspektive mit einem Lernen über Medien verstanden?

- Wie kann ein Lernen über Medien angeregt werden?

Zur Beantwortung dieser Fragen wird im ersten Schritt das Konstrukt der Medienkompetenz als Zielstellung für medienpädagogisches Handeln konkretisiert und damit verbundene Aufgabenbereiche skizziert (Kap. 4.5.1.1). Der zweite Schritt widmet sich aktuellen Konzepten zur Gestaltung medienpädagogischer Praxis als handlungsorientierte Medienerziehung (Kap. 4.5.1.2). 


\subsubsection{Medienkompetenz als Ziel und Aufgabenbereiche der Medienbildung}

Der Begriff der Medienkompetenz wird in zahlreichen Kontexten und disziplinären Zusammenhängen diskutiert (Gapski 2001). Im Rahmen der begrifflich-theoretischen Positionierung wurde Medienkompetenz bisher vor allem als zentraler Bestandteil medienpädagogischer Kompetenz skizziert (Kap. 4.3.2.4). Mit der Verbreitung des Medienkompetenzbegriffs über vielfältige Kontexte und Diskurse ist es - analog zum Begriff der Medienbildung (Kap. 4.3.1) - jedoch nicht überraschend, dass die Bedeutung des Begriffs variiert und für unterschiedliche Zusammenhänge adaptiert wird. So erscheint es für das vorliegende Projekt sinnvoll, näher zu konkretisieren, was im Folgenden unter Medienkompetenz verstanden wird.

Als Schlüsselbegriff des medienpädagogischen Fachdiskurses sowie als zentraler Diskussionsgegenstand verschiedener Fachtagungen (Herzig et al. 2010; Moser et al. 2011) kann zur Konkretisierung des Medienkompetenzbegriffs auf zahlreiche Begriffsdiskussionen zurückgegriffen werden (z. B. Herzig und Grafe 2010; Schaumburg und Hacke 2010; Gapski 2001) An diesen Diskussionsstand anknüpfend werden zentrale Merkmale des Medienkompetenzbegriffs aus einer forschenden sowie aus einer praxisbezogenen Perspektive benannt. Aus der Perspektive der empirischen Bildungsforschung zur Messung von Medienkompetenz kommen Schaumburg und Hacke bei vergleichender Betrachtung unterschiedlicher Ansätze zu folgenden Aussagen:

- «Es handelt sich um ein mehrdimensionales Konstrukt, dessen Teilkomponenten aufgrund ihrer Verankerung in unterschiedlichen Disziplinen in ihrer Binnenstruktur auf ein heterogenes Gerüst von Teiltheorien zurückgreifen» (Schaumburg und Hacke 2010, 150).

- «Bezüglich zentraler Komponenten des Konstrukts stimmen unterschiedliche Konzepte überein, im Detail weichen die Konzepte allerdings voneinander ab» (Schaumburg und Hacke 2010, 151).

- «Zur Beziehung der Teilkomponenten gibt es bisher keine einheitlichen Vorstellungen» (ebd.).

- «Die pädagogische Tradition des Konstrukts resultiert in einer normativen Ausrichtung von Medienkompetenz als Kompetenz zur gesellschaftlichen Teilhabe» (ebd.).

Die erste Aussage von Schaumburg und Hacke ist anschlussfähig an die Überlegungen von Sutter (2010) sowie Herzig und Grafe (2010). So skizzieren sie verschiedene kompetenztheoretische Forschungstraditionen und disziplinäre Perspektiven, die der Diskussion um das Konstrukt Medienkompetenz vorausgehen. Frühere sprachwissenschaftliche bzw. linguistische Bestimmungen des Kompetenzbegriffs können nach Sutter (2010, 44 f.) beispielsweise von «strukturgenetischen Kompetenztheorien» unterschieden werden. Damit verbunden sind jeweils unterschiedliche Annahmen für das Konstrukt der Medienkompetenz. 
Für die empirische Erfassung von Medienkompetenz werden von Schaumburg und Hacke $(2010,151 \mathrm{ff}$.) in diesem Zusammenhang verschiedene Probleme und Herausforderungen skizziert. Aufgrund der Mehrdimensionalität des Medienkompetenzbegriffs besteht in der Reduktion des Konstruktes das Problem einer unzulässigen Vereinfachung oder funktionalistischen Verengung. Sutter $(2010,46$.$) weist ferner$ auf das Methodenproblem hin, dass «nur Performanzen direkt empirisch zugänglich sind und aus diesen Daten indirekt auf Kompetenzen geschlossen werden muss». Darüber hinaus kann in Anlehnung an Sutter $(2010,46$.) eine quantitativ-empirische Forschungsperspektive auf Medienkompetenz für die praxisbezogene Entwicklung und Realisierung konkreter Lehrveranstaltungen insofern problematisiert werden, dass Medienkompetenz «überwiegend in Form statisch feststellbarer Lernresultate» formuliert wird. Die Erfassung von Lernprozessen im Sinne des Erwerbs von Medienkompetenzen stellt, so Sutter (2010), jedoch die deutlich schwierigere Fragestellung dar, welche jedoch kaum bearbeitet wird.

Neben der weitgehend abstrakten Charakterisierung des Medienkompetenzbegriffs aus der Sicht der empirischen Bildungsforschung von Schaumburg und Hacke (2010) lassen sich nach Herzig und Grafe (2010) weitere Eigenschaften formulieren. Mit der Zielperspektive, Möglichkeiten zur Entwicklung von Bildungsstandards für die Medienbildung zu formulieren, kommen Herzig und Grafe $(2010,108)$ beim Vergleich unterschiedlicher Ansätze zu folgenden Aussagen über das Konstrukt der Medienkompetenz:

- "Medienkompetenz rekurriert auf die kommunikative Deutung des anthropologischen Grundverhältnisses des Menschen zu sich selbst, zu einer dinglichen und sozialen Umwelt, bezieht dabei aber den Menschen als Ganzen mit seinen kognitiven, ästhetischen, affektiven und moralischen Bedürfnissen ein. Sie ist damit konstitutiver Bestandteil der Entwicklung von Weltsichten, von sozialen Beziehungen und der Entwicklung der eigenen Persönlichkeit. Medienkompetenz hat damit nicht eine primär funktionale Bedeutung, sondern eine bildende.

- Medienkompetenz ist ausgerichtet auf ein sachgerechtes, selbst bestimmtes, kreatives und sozial-verantwortliches Handeln in einer von Medien geprägten Welt und damit wichtiger Bestandteil handelnder Lebensbewältigung.

- Medienkompetenz bezieht sich auf verschiedene Handlungsforderungen im Kontext von Medien, z. B. die Erkundung medialer Räume, die Gestaltung medialer Produkte, die Beurteilung von Mediengestaltungen oder von Entwicklungen im Medienbereich, das Treffen von Entscheidungen im Medienzusammenhang oder die Problemlösung unter Einbeziehung von Medien» (Herzig und Grafe 2010, 108).

Die von Herzig und Grafe $(2010,108)$ formulierten Eigenschaften von Medienkompetenz lassen sich als inhaltliche Konkretisierung der Aussage von Schaumburg und Hacke $(2010,152)$ verstehen, dass unterschiedliche Konzepte hinsichtlich der 
zentralen Komponenten übereinstimmen. Zudem wird in den Aussagen von Herzig und Grafe $(2010,108)$ in unterschiedlicher Weise die von Schaumburg und Hacke (2010) benannte Mehrdimensionalität des Konstruktes erkennbar, welche sich hinsichtlich der bildenden Bedeutung, der normativen Ausrichtung sowie der verschiedenen Handlungsforderungen zeigt. Entgegen der Kritik von Sutter (2010), dass der Medienkompetenzbegriff vor allem statische Lernresultate statt Lernprozesse fokussieren würde, formulieren Herzig und Grafe $(2010,108)$ zudem folgende Annahmen zum Erwerb bzw. zur Entwicklung von Medienkompetenz:

- «Medienkompetenz ist in ihrer jeweils aktuellen Erscheinungsform entwicklungsbedingt und - in erzieherischer Hinsicht - entwicklungsfähig.

- Medienkompetenz kann in handelnder Auseinandersetzung des Individuums mit medienbezogenen Aufgaben und Inhalten in sich aktiv aneignender Weise (weiter-)entwickelt werden» (Herzig und Grafe 2010, 108).

Während die erste Prämisse die Grundlage für pädagogische Tätigkeiten zur Förderung von Medienkompetenz darstellt, konkretisiert die zweite Annahme die Art und Weise der Aneignung von Medienkompetenz. Diese Grundannahmen lassen sich in der aktuellen Position einer handlungsorientierten Medienpädagogik verorten (Kap. 4.5.1.2). Die von Herzig und Grafe $(2010,108)$ benannten «Aufgaben und Inhalte» können exemplarisch in Anlehnung an Tulodziecki et al. (2010) strukturiert werden. So unterscheiden Tulodziecki et al. $(2010,180)$ zum einen zwei grundlegende Handlungszusammenhänge mit Medien: (1.) «die Auswahl und Nutzung vorhandener medialer Angebote» und (2.) «die Gestaltung und Verbreitung eigener medialer Beiträge». Diese Handlungszusammenhänge können nach Tulodziecki et al. $(2010,180)$ sowohl in getrennter Form (z. B. die Auswahl eines spezifischen Fernsehprogramms) als auch in gemeinsamer Form auftreten (z. B. bei der Online-Kommunikation über soziale Medien). Zudem zeichnet sich Medienkompetenz für Tulodziecki et al. (2010, 180) durch «Kenntnisse und Analyse- sowie Urteilsfähigkeit» aus. Diese werden hinsichtlich drei verschiedener Inhaltsbereiche unterschieden: (1.) «Möglichkeiten der Mediengestaltung», (2.) «Medieneinflüsse» und (3.) «Bedingungen der Medienproduktion und Medienverbreitung». Diese unterscheidbaren Handlungs- und Inhaltsbereiche stehen in Beziehung zueinander (Abb. 4.7). 


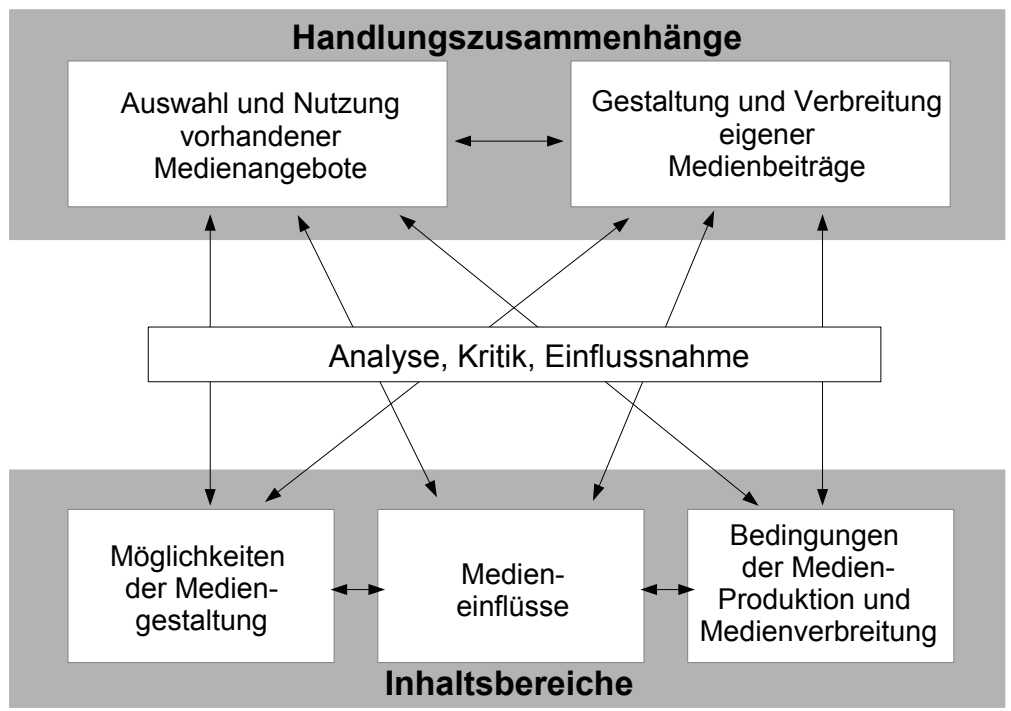

Abb. 4.7: Schematische Darstellung zur «Strukturierung des Konzepts von Medienkompetenz» von Tulodziecki et al. $(2010,181)$.

Auf Basis dieser ersten Unterscheidung differenzieren Tulodziecki et al. (2010, 182) «fünf Aufgabenbereiche für die Förderung von Medienkompetenz bzw. für die Medienbildung». Diese umfassen: «Auswählen und Nutzen von (vorhandenen) medialen Angeboten, Gestalten und Verbreiten eigener medialer Beiträge, Verstehen und Bewerten von Mediengestaltungen, Erkennen und Aufarbeiten von Medieneinflüssen, Durchschauen und Beurteilen von Bedingungen der Medienproduktion und Medienverbreitung» (Tulodziecki et al. 2010, 182). In Anlehnung an dieses allgemeine Strukturierungsmodell konkretisiert Tulodziecki $(2012,275)$ in einem Entwurf von Medienbildungsstandards zudem spezifische Kompetenzerwartungen für die einzelnen Aufgabenbereiche. Demnach sollten medienkompetente Lehramtsstudierende sowie Lehrerinnen und Lehrer «in der Lage und bereit sein»,

- «Medienangebote und nicht-mediale Möglichkeiten im Hinblick auf angestrebte Nutzungszusammenhänge zu erläutern und sachgemäß zu handhaben, kriterienbezogen zu vergleichen und zu bewerten, begründet auszuwählen sowie unter Beachtung sozialer bzw. gesellschaftlicher Verantwortung zu nutzen,

- eigene Aussagen unter Verwendung bewusst ausgewählter Gestaltungsarten mit sachgemäßer Handhabung der jeweiligen Technik und situationsangemessener Planung sowie unter Beachtung sozialer bzw. gesellschaftlicher Verantwortung zu gestalten und an Einzelne, bestimmte Gruppen oder öffentlich zu verbreiten,

- Gestaltungsmöglichkeiten von Medien zu erläutern und in ihrer Bedeutungfür mediale Aussagen einzuschätzen sowie Gestaltungsmittel für eigene Medienbeiträge begründet auszuwählen und die eingesetzten Gestaltungsmittel 
in vorhandenen Medienangeboten analytisch zu erfassen und hinsichtlich der Übereinstimmung von Form und Inhalt oder anderer Kriterien bewerten,

- Medieneinflüsse zu beschreiben, kriterienbezogen zu bewerten und problematische Einflüsse der Mediennutzung in geeigneten Formen aufzuarbeiten und ihnen entgegenzuwirken sowie Medieneinflüsse bei der eigenen Mediengestaltung und Verbreitung zu berücksichtigen,

- Bedingungen von Medienproduktion und Medienverbreitung zu erläutern, Verbindungen zwischen solchen Bedingungen und den Medienprodukten sowie ihrer Nutzung herzustellen, die Bedingungen in Orientierung am gesellschaftlich Wünschenswerten zu beurteilen und Einflussmöglichkeiten beim eigenen Handeln zu skizzieren und wahrzunehmen» (Tulodziecki 2012, 276).

Die von Tulodziecki $(2012,276)$ formulierten Ziel- und Prozessbeschreibungen lassen sich als eine exemplarische Modellierung des Kompetenzbereiches «eigene Medienkompetenz» des bereits skizzierten Konstruktes der «medienpädagogischen Kompetenz» (Blömeke 2000, 2005) verstehen. Dabei betont Tulodziecki $(2012,277)$, dass nicht zu erwarten ist, «dass Lehrpersonen Experten für alle Medienarten sind». Stattdessen "geht es vor allem», so Tulodziecki (2012, 277), «um Grundkenntnisse und grundlegende Fähigkeiten bezogen auf wichtige Teilaufgaben». Für den Entwurf konkreter Lehrveranstaltungen (Kap. 6) sind auch die Ziel- und Prozessbeschreibungen von Tulodziecki $(2012,276)$ zur Konkretisierung der allgemeinen Medienkompetenz hilfreiche Orientierungs- und Strukturierungsmöglichkeiten. Ferner lassen sich anhand des Strukturierungsvorschlags die spezifischen Themen- und Fragestellung in der Auseinandersetzung mit sozialen Medien verorten (Kap. 4.5.2).

\subsubsection{Handlungsorientierung als Leitidee praktischer Konzepte zur Medienziehung}

Eine Beantwortung der Fragestellung, wie ein Lernen über Medien angeregt werden kann, erfolgt in der medienpädagogischen Fachdebatte $u$. a. durch Konzepte zur Medienerziehung. Zugleich haben sich die Konzepte der Medienerziehung sowie die Strömungen der Medienpädagogik im 20. Jahrhundert mehrfach deutlich verändert. In zahlreichen medienpädagogischen Überblicksartikeln und Handbuchbeiträgen wird diese Veränderung und Erweiterung von Positionen und Konzepten beschrieben (z. B. Sander et al. 2008; Spanhel 2006; Tulodziecki et al. 2010). Auffällig ist dabei zum einen, dass sich diese Systematisierungsversuche hinsichtlich der Bezeichnungen früherer Phasen durchaus unterscheiden. Zum anderen ist diesen Ausführungen gemeinsam, dass die Bedeutung einer handlungsorientierten Medienerziehung bzw. Medienpädagogik im aktuellen Diskurs betont wird (Spanhel 2006; Schorb 2008; 
Tulodziecki et al. 2010) ${ }^{122}$. An den vorgestellten Überlegungen anknüpfend, wird im Folgenden konkretisiert, was unter Handlungsorientierung als Leitidee und Gestaltungsprinzip verstanden werden kann. Dies erfolgt exemplarisch für die Überlegungen von Schorb (2008) für die ausserschulische Jugendarbeit sowie in Anlehnung an Tulodziecki et al. (2010) für das Handlungsfeld Schule.

Die Position einer handlungsorientierten Medienpädagogik basiert auf der Annahme, dass sich Menschen aktiv mit Medien auseinandersetzen können und nicht als blosse Rezipientinnen und Rezipienten von Medien zu betrachten sind. Entsprechend stehen nicht Medien im Mittelpunkt dieser Position, sondern - vereinfacht formuliert - Menschen in Auseinandersetzung mit Medien. Nach Schorb $(2008,77)$ sind es «die aneignenden Individuen in ihrem gesamtgesellschaftlichen Kontext, in dem Medien eine wesentliche Rolle spielen», die im Mittelpunkt einer handlungsorientierten Medienpädagogik stehen. Die Berücksichtigung des gesellschaftlichen und sozialen Kontextes ist $u$. a. deshalb relevant, weil die Nutzung von Medien als «soziales Handeln» verstanden wird (Tulodziecki et al. 2010, 166). In diesem Zusammenhang wird Menschen bei der aktiven Mediennutzung eine «Entscheidungsfreiheit» zugesprochen und zugleich wird von Tulodziecki et al. $(2010,166)$ einschränkend formuliert, dass Menschen «konventionellen Regeln und strukturellen Bedingungen» folgen. So wäre es nach Tulodziecki et al. $(2010,166)$ eine Illusion zu glauben, «das Individuum könne in jedem Fall beliebig und souverän - nur gemäß den eigenen Interessen - Medien nutzen». Stattdessen kann von einem Wechselverhältnis zwischen Individuen, Medien und dem sozialen Kontext ausgegangen werden:

«Das Individuum wendet sich mit bestimmten Bedürfnissen, Erwartungen und Vorstellungen den Medien zu, die Medien bieten jedoch nur bestimmte vorgeformte Möglichkeiten und wirken in ihrer Gesamtheit auf die Bedürfnisse,

122 Spanhel $(2006,288)$ beginnt seine Charakterisierung der «Entwicklung medienpädagogischer Positionen» beispielsweise mit der Beschreibung einer «kulturkritisch-geisteswissenschaftlichen Position». Diese als bewahrpädagogische charakterisierte Position verortet Spanhel $(2006,288)$ seit Beginn des 20. Jahrhunderts bis in die 1960er-Jahre. Im Fokus der aktuellen Diskussion steht für Spanhel (2006, 230) die «Handlungsorientierte Position», welche die Bedeutung praktischer Medienarbeit als medienerzieherisches Konzept betont. Ein zweites Beispiel für eine ähnliche historische Rekonstruktion bieten die Ausführungen von Tulodziecki et al. (2010). Mit der Formulierung einer «behütend-pflegende[n] Medienziehung» bezeichnen Tulodziecki et al. $(2010,157)$ pädagogische Konzepte "zum Ende des 19. und zum Beginn des 20. Jahrhunderts». Nach der Vorstellung von und der Kritik an weiteren Positionen präsentieren Tulodziecki et al. (2010) Konzepte einer «Handlungs- und interaktionsorientierten Medienerziehung» seit den 1980er Jahren als Weiterentwicklung früherer Vorstellungen. Dabei sind «Grundgedanken einer handlungs- und interaktionsorientierten Medienerziehung» auch für aktuelle Entwürfe und Konzepte «zur Medienerziehung bzw. Medienbildung bedeutsam geblieben» (Tulodziecki et al. 2010). So bezeichnen Tulodziecki et al. (2010) auch ihr eigenes Konzept als «handlungs- und entwicklungsorientierte Medienbildung». Ein drittes Beispiel für eine entsprechende historische Darstellung liefert das Handbuch Medienpädagogik (Sander et al. 2008). Das Kapitel zur «Geschichte und Strömungen der Medienpädagogik» beginnt mit einem Beitrag zur Rolle von Medien im Diskurs der Reformpädagogik zum Ende des 19. Jahrhunderts bzw. zu Beginn des 20. Jahrhunderts (Moser 2008). Den Abschluss des Kapitels bildet der Beitrag «Handlungsorientierte Medienpädagogik» von Schorb (2008). Dabei skizziert Schorb (2008) zentrale Aspekte des Konzeptes und markiert die Relevanz handlungs- und produktorientierter Medienarbeit für unterschiedliche Handlungsfelder. 
Vorstellungen und Erwartungen ihrer Nutzer zurück. Dabei ist die Mediennutzung in den jeweiligen Lebenskontext von Individuen und sozialen Gruppen eingebettet» (Tulodziecki et al. 2010, 166).

Das Ziel medienpädagogischer Handlungen im Sinne einer handlungsorientierten Medienpädagogik kann allgemein als Vermittlung von Medienkompetenz verstanden werden. Medienkompetenz wird in diesem Zusammenhang als «Bestandteil kommunikativer Kompetenz» (Tulodziecki et al. 2010, 168) betrachtet bzw. nach Schorb (2008) als zeitgemässe Präzisierung der «Zielsetzung der Vermittlung kommunikativer Kompetenz» verstanden ${ }^{123}$. Mit dem Begriff der «kommunikativen Kompetenz» verweisen sowohl Tulodziecki et al. (2010) als auch Schorb (2008) auf die Arbeiten von Baacke (z. B. 1973, 1997). Dabei betont Schorb $(2008,78)$, dass die Zielsetzung Medienkompetenz zwar einerseits «allgemein gültig» ist. Anderseits ist die Konkretisierung bzw. Operationalisierung von Medienkompetenz nach Schorb (2008, 78) jeweils «an den Adressaten auszurichten». Die Adressatinnen und Adressaten des vorliegenden Forschungs- und Entwicklungsprojektes sind Lehramtsstudierende. Daher erfolgte eine Darstellung medienpädagogischer Kompetenzmodelle für Lehrerinnen und Lehrer und Lehramtsstudierende im Rahmen der begrifflich-theoretischen Positionierung (Kap. 4.3.2).

Auf Basis der skizzierten Grundannahme und Zielsetzung handlungsorientierter Medienpädagogik formuliert Schorb (2008, 80 f.) sein Konzept einer «reflexivpraktischen Medienarbeit», welches als spezifisch für «pädagogische Prozesse mit Jugendlichen» geschildert wird. Die Ausführungen beinhalten eine Konkretisierung des Medienkompetenzbegriffs sowie spezifischer Zielsetzungen «reflexiv-praktischer Medienarbeit» (Schorb 2008, 78 ff.). Medien werden in diesem Konzept von Schorb $(2008,82)$ als «Mittel der Darstellung eigener Meinungen zu Themen und Problemen» sowie als «Mittel der Aufarbeitung und Vermittlung eigener Erfahrungen» verstanden. Für die Gestaltung von Lehr- und Lernsituationen ist für Schorb $(2008,83)$ entscheidend, dass «in all diesen aktiven Verwendungsnachweisen von Medien der Prozess der Herstellung eines medialen Produktes» von Bedeutung ist. Im Rahmen dieser gestalterischen Auseinandersetzung finden verschiedene Lernprozesse statt. So nennt Schorb $(2008,83)$ u. a. die folgenden Möglichkeiten «Wissen zu inhaltlichen Bereichen kann vertieft oder neu erworben werden», «Erfahrungen können durch das eigene Handeln in Prozessen der Herstellung neu gemacht werden» und «eigene Handlungsorientierungen können reflektiert, differenziert, neu erworben oder gestärkt werden». Um entsprechende Lernprozesse erfolgreich anzuregen, skizziert Schorb (2008, 83 f.) drei pädagogische Voraussetzungen und Prinzipien. Unter Berücksichtigung dieser Aspekte kann die praktisch-reflexive Medienarbeit sowie damit

123 Schorb $(2008,78)$ begründet seine Position mit dem Argument, «dass mediale Kommunikation zum zentralen Modus der Verständigung in der Gesellschaft geworden ist». 
verbunden Lernprozesse «einen Beitrag zur eigenständigen Lebensbewältigung und gesellschaftlichen Partizipation der Adressaten leisten» (Schorb 2008, 84). Die Voraussetzungen und Prinzipien für die Gestaltung von Lehr- und Lernsituationen im Sinne der praktisch-reflexiven Medienpädagogik beschreibt Schorb $(2008,83$ f.) wie folgt:

- «Reflexiv-praktische Medienarbeit setzt an den Bedingungen und Voraussetzungen ihrer Adressaten an. [...] Das heißt, Ausgangspunkt von Prozessen reflexiv-praktischer Medienarbeit sind deren Interessen, Problemlagen, Erfahrungen. Diese bestimmen das Thema. Die Umsetzung des Themas orientiert sich an den alltagspraktischen Verarbeitungs- und Handlungsorientierungen der Adressaten.

- Reflexiv-praktische Medienarbeit ist an Selbsttätigkeit und Eigenverantwortung der Adressaten zu orientieren. Die Herstellung eines medialen Produktes ist ein Prozess, den die pädagogischen Subjekte weitgehend selbst in der Hand haben sollen. Sie sollen Organisation und Verlauf möglichst eigenverantwortlich bestimmen. Der/die PädagogIn ist UnterstützerIn und RatgebenerIn.

- Reflexiv-praktische fördert insbesondere die Fähigkeit zur inhaltlichen Nutzung der Medien als Mittel und Mittler von Kommunikation. [...] Die inhaltliche Auseinandersetzung mit einem Themenbereich im Herstellungsprozess und die dabei bei den Adressaten in Gang gesetzten Lern- und Erfahrungsprozesse sind wesentlicher als ein technisch perfekt umgesetztes und gestaltetes Produkt» (Schorb 2008, 83 f.).

Mit dem Fokus auf den Lernort Schule und dessen spezifische Bedingungen hat Tulodziecki (1992) ein Konzept für die schulische Medienerziehung bzw. haben Tulodziecki et al. (2010) ein Konzept für schulische Medienbildung entworfen. Die zentrale Zielstellung dieses Konzeptes wurde bereits im Rahmen des Kapitels als Aufgabenbereiche und Kompetenzerwartungen konkretisiert (Kap. 4.5.1.1). Tulodziecki et al. $(2010,66)$ erklären das Konzept der Handlungsorientierung als übergeordnetes didaktisches Prinzip für medienpädagogisches Handeln: «Medienbildung soll für gegenwärtiges und zukünftiges Handeln bedeutsam sein und handlungsbezogen erfolgen» (ebd.). Dieses Prinzip verknüpfen Tulodziecki et al. (2010, 66 f.) mit sechs weiteren didaktischen Leitideen und Prinzipien:

- «Sinnorientierung: Ausgangspunkt für medienpädagogisches Handeln sollen Situationen aus der Lebenswelt von Kindern und Jugendlichen sein, und das zu Lernende soll auf jetzige und zu erwartende Lebenssituationen bezogen werden.

- Kommunikationsorientierung: Lernen und Erziehung sollen in Kommunikation und für Kommunikation mit Anderen erfolgen, z. B. indem eigene Medienerfahrungen, Interessen und Bedürfnisse personal oder mithilfe von Medien 
mitgeteilt und personale oder technisch übertragene Mitteilungen Anderer angemessen aufgenommen werden.

- Bedürfnisorientierung: Diese Orientierung meint zunächst, dass die Bedürfnisse von Kindern und Jugendlichen bei der Mediennutzung ernst genommen und akzeptiert werden. Bedürfnisorientierung meint darüber hinaus, dass die erzieherischen Prozesse so gestaltet werden, dass in ihnen Bedürfnisse der Kinder und Jugendlichen zum Tragen kommen.

- Erfahrungsorientierung: Ausgangspunkt für Lernen und erzieherische Aktivitäten sollen inhaltliche und mediale Erfahrungen der Kinder und Jugendlichen sein, im Laufe erzieherischer Prozesse sollen neue inhaltliche und mediale Erfahrungen ermöglicht werden. [...]

- Unter dem Aspekt Entwicklungsorientierung ist es wichtig, dass Lernen und die erzieherische Anregung sowie die Unterstützung von Kindern und Jugendlichen einerseits entwicklungsgemäß erfolgen und anderseits entwicklungsfördernd wirken.

- Kompetenzorientierung besagt, dass für medienpädagogische Prozesse Medienkompetenz als generelles Vermögen des Menschen, in Medienzusammenhängen sinngerecht zu handeln, vorausgesetzt wird und es gleichzeitig um eine Förderung entsprechender Fähigkeiten und Bereitschaften geht» (Tulodziecki et al. 2010, 66 f.).

Zur begründeten Entwicklung von Lehr- und Lernveranstaltungen für Lehramtsstudierende (Kap. 6) dienen die vorgestellten Prinzipien und Voraussetzungen für ein angemessenes medienpädagogisches Handeln zur Förderung der Medienkompetenz - neben den skizzierten mediendidaktischen Erkenntnissen (Kap. 4.4) - als relevante Strukturierungs- und Orientierungshilfen. Zur spezifischeren Auseinandersetzung mit den Eigenschaften sozialer Medien (Kap. 4.3.3) wird im Folgenden ein Einblick in den Diskurs zentraler Themen zu sozialen Medien skizziert.

\subsubsection{Soziale Medien als Thema und Erfahrungsraum}

In Auseinandersetzung mit medienpädagogischen Zielstellungen wird der Gegenstandsbereich einer medienpädagogischen Professionalisierung auf das Thema «soziale Medien» fokussiert (Kap. 4.3.3). Diese Fokussierung erfolgt in Anlehnung an die vorgestellten Kompetenzmodelle von Mayrberger (2012a) und Moser (2010b) (Kap. 4.3.2). Zudem erfolgt die Fokussierung auf soziale Medien mit Verweis auf das Themenheft «Social Media in der Lehrerbildung» im «Journal für LehrerInnenbildung» (Mayrberger et al. 2013; Schiefner-Rohs 2013b; Spannagel 2013). In Anknüpfung an eine medienerzieherischen Perspektive widmen sich die folgenden Überlegungen der Frage, für welche aktuellen Themen und Fragen Lehramtsstudierende in der 
Auseinandersetzung mit sozialen Medien sensibilisiert werden können. Zur Beantwortung dieser Frage werden zwei exemplarische Zukunftsargumente vorgestellt. Zukunftsargumente lassen sich in Anlehnung an Döbeli Honegger (2016) dahingehend verstehen, dass Kompetenzen erworben werden sollen, die für das zukünftige Handeln der Menschen relevant sind. Dabei werden exemplarisch die folgenden zwei Aspekte fokussiert: (a) die Fähigkeit zur Teilnahme und Teilhabe an einer partizipativen Kultur (Kap. 4.5.2.1) und (b) der reflexive Umgang mit der Veränderung von Privatheit und informationeller Selbstbestimmung (Kap. 4.5.2.2).

\subsubsection{Teilnahme und Teilhabe an einer partizipativen Kultur}

Eine zentrale These in der medienpädagogischen und mediendidaktischen Fachdiskussion über soziale Medien lautet, dass diese neuen Partizipationsmöglichkeiten eröffnen können sowie das Potenzial haben, die Entwicklung von Fähigkeiten zur Teilnahme und Teilhabe an einer partizipativen Kultur zu fördern. In Betrachtung verschiedener Diskurszusammenhänge wird diese These im Folgenden belegt und hinsichtlich des jeweiligen Partizipationsverständnis konkretisiert. Bei vergleichender Betrachtung der Debatten über E-Learning-2.0 und einer Medienbildung für die Schule sowie neuer Formen politischer Teilhabe lassen sich verschiedene Schwerpunkte markieren.

In der deutschsprachigen E-Learning-2.0-Diskussion (Kap. 4.4.2) wird in unterschiedlichen Zusammenhängen auf den Partizipationsbegriff sowie die Partizipationspotenziale Bezug genommen. Beispielsweise verwendet Ehlers (2010) den Partizipationsbegriff als Metapher für das zentrale Lernverständnis im E-Learning-2.0-Diskurs. In ähnlicher Weise wird Partizipation in verschiedenen Beiträgen als relevantes - wenngleich nicht als das zentrale - Charakteristikum von neuen Lehr- und Lernkonzepten im Umgang mit digitalen Medien verstanden (z. B. Barth 2008; Ehlers 2010; Mayrberger 2010b). So lässt sich E-Learning-2.0 nach Mayrberger (2010b, 311) «allgemein durch einen hohen Grad an Interaktivität, Selbstorganisation, Partizipation, Kooperation bzw. Kollaboration, Communitiy-Orientierung und Nutzerorientierung charakterisieren». Ferner wird Partizipation als Charakteristika des Web 2.0 respektive der sozialen Medien im Zusammenhang mit neuen Möglichkeiten bzw. Annahmen zur veränderten Nutzungsweisen thematisiert (z. B. bei Mayrberger 2010b; Kimpeler 2010; Schiefner 2011). Beispielsweise skizziert Schiefner (2011, 309) die im Diskurs existierende Perspektive wie folgt: «Studierende nutzen die Medien nun nicht mehr nur passiv, sondern es besteht die Möglichkeit zur aktiven Teilhabe und Partizipation, zur Vernetzung von Personen, zur Kollaboration; [...]». Im Vergleich der angegebenen Diskussionsbeiträge erscheint das jeweilige Partizipationsverständnis auch auf anderen Ebenen verortet zu sein: (1.) als Bedingung (z. B. Bader 2010; Mayrberger 
2010b), (2.) als Interaktion zwischen Akteurinnen und Akteuren (z. B. Appelt 2011; Kimpeler 2010) oder (3.) als Erklärungsansatz für Lernen (z. B. Barth 2008).

Mit Blick auf den englischsprachigen Diskurs über E-Learning-2.0 und der Verwendung des Begriffs «participation» wird - vermutlich auch bedingt durch die Übersetzung - eine noch engere Perspektive identifiziert. So wird der Begriff «participation» mitunter als synonymer Begriff für Lernen (im konstruktivistischen Sinne) verwendet (z. B. Kim et al. 2013; Papastergiou et al. 2011; Waycott et al. 2013). Exemplarisch veranschaulichen lässt sich diese Begriffsverwendung an der Formulierung von Papastergiou et al. $(2011,1999):$ «[...] the affordances offered by blogs [...] can serve social constructivist approaches to learning that emphasize students' active participation in knowledge construction through social interactions». Ferner wird der Begriff verwendet, um über die Nutzung bestimmter Funktionalitäten von sozialen Medien zu berichten: «Students reported participation in a variety of Facebook activities, with (viewing photos), (commenting on content) and (checking to see what someone is up to being the three most popular activities» (Junco 2012, 191). Die Bandbreite an unterschiedlichen Verständnissen veranschaulicht, dass die Verwendung des Partizipationsbegriffs in der Debatte ein relevantes Thema darstellt und zugleich unterschiedlich akzentuiert wird.

Ein weiteres Begriffsverständnis findet sich in der Diskussion um schulische Medienbildung. Die Fähigkeiten Teilnahme und Teilhabe an einer partizipativen Kultur zu entwickeln, stellt für Moser (2010b, 72) ein zentrales Bildungsziel für die Schule dar, welches sich auch auf die Hochschule übertragen lässt. Mit Bezugnahme auf die Argumentation von Jenkins et al. (2009) ${ }^{124}$ formuliert Moser, dass es das Ziel von Bildungsinstitutionen mit jungen Menschen sein sollte, «die erforderlichen Fähigkeiten, das nötige Wissen, ethische Werte und Selbstvertrauen zu entwickeln, um vollwertige Teilnehmer dieser Kultur zu werden» (Moser 2010b, 73). Die Relevanz dieses Zukunftsarguments zur Auseinandersetzung mit sozialen Medien kann in erster Annäherung mit dem Partizipationsbegriff begründet werden. So ist für Mayrberger und Moser (2011) Partizipation «neben Mündigkeit und Emanzipation ein genuin pädagogisches Anliegen und wichtige Antriebskraft einer handlungs- und interaktionsorientierten Medienpädagogik». Dabei machen u. a. Mayrberger und Moser (2011), Biermann et al. (2014) sowie Kammerl und Unger (2014) deutlich, dass mit dem aktuellen Medienwandel und der Verbreitung digitaler Medien neue diskursive Räume entstehen. Diese «neue[n] Beteiligungsformen» (Mayrberger und Moser 2011) bzw. «veränderte[n] Formen öffentlicher Teilhabe»(Biermann et al. 2014) sowie

124 Moser (2010b) verweist in seinem Text auf eine Publikation von Henry Jenkins aus dem Jahr 2006, welche allerdings im Literaturverzeichnis nicht angegeben ist. Mit Blick auf das von Moser (2010b, 72 f.) übersetzte Zitat «Eine partizipative Kultur entsteht dadurch, dass die Kultur auf die Explosion der neuen Medientechnologien reagiert und diese absorbiert [...]» kann begründet davon ausgegangen werden, dass Moser (2010b) auf die Publikation von Jenkins et al. (2009) verweist, welche hier in einer überarbeiteten Fassung referenziert wird. 
«diskursiver und produktiver Praktiken» (Kammerl und Unger 2014) werfen zugleich neue Fragen auf und führen zu neuen Herausforderungen. Als Gemeinsamkeit dieser Positionen lässt sich die Erkenntnis formulieren, dass die digitale Kultur des Internets, des Web 2.0 bzw. der sozialen Medien eng mit unserer «alltäglichen Lebenswelt [...] verflochten ist» (Mayrberger und Moser 2011, o. S.) und zu einer «Transformation individueller wie gesellschaftlicher Lebensbereiche [führt]» (Kammerl und Unger 2014, 6). Mayrberger und Moser (2011) erläutern dies exemplarisch wie folgt:

«So wird das Netz, wo man online einkauft, Freunde im Chat trifft, sich täglich über aktuelle Nachrichten informiert, immer mehr als Erweiterung des alltäglichen Lebens betrachtet. Dies bedeutet daher auch, dass wer am Netz aktiv partizipiert, zugleich über einen Anteil an gesellschaftlicher Macht verfügt. Politische Kampagnen im Internet oder die Präsentation von Politikern/-innen bei Wahlkämpfen im Netz unterstreichen diesen Trend auf eindrückliche Weise» (Mayrberger und Moser 2011, o. S.).

Neben der Erkenntnis, das Internet - und damit auch soziale Medien - als Kulturraum zu verstehen, lässt sich als zweite Gemeinsamkeit die Perspektive markieren, dass im Kontext des Web 2.0 «jede/r potenziell ein (Prosumer), also Produzent/in und Konsument/in in einem [ist]» (Mayrberger und Moser 2011, o. S.). Ähnlich wie bei der skizzierten E-Learning-2.0-Debatte (Kap. 4.4) argumentieren beispielsweise auch Mayrberger und Moser (2011), dass sich mehr Menschen «dank technisch niedrigschwelliger Softwareangebote potenziell am «Mitmachnetz〉 beteiligen». Dabei werden Inhalte, so Mayrberger und Moser (2011) weiter, «von Einzelnen oder kollaborativ im Netz erstellt und publiziert, (ausgewählt) rezipiert und weiterpubliziert [...] Das Internet wird so zu einem wesentlichen Medium der Partizipation». Auch Biermann et al. (2014) argumentieren, dass die «Trennlinie zwischen Produzenten und Rezipienten, zwischen Medienmachern und Mediennutzern» zunehmend verwischt. Als Antrieb dieser Entwicklung sowie der Auflösung zeitlich und räumlicher Grenzen markieren Biermann et al. (2014) die «stetig steigende mobile Netznutzung».

Um die Relevanz der von Moser (2010b) und Jenkins et al. (2009) formulierten Zielstellung zu stützen, wird der Partizipationsbegriff als genuin-pädagogisches Anliegen skizziert. Gleichwohl wird der Partizipationsbegriff in aktuellen bildungs- und sozialwissenschaftlichen Diskursen keinesfalls einheitlich verwendet. Biermann et al. (2014) verweisen in ihrer einleitenden Betrachtung von «partizipativen Medienkulturen als Transformation von Beteiligungsmöglichkeiten» beispielsweise auf den Partizipationsbegriff von Habermas (1992). Politische Partizipation realisiert sich bei Habermas, so Biermann et al. $(2014,8)$, als «vor allem in der Beteiligung am öffentlichen Diskurs über die Gesellschaft relevanten (politischen) Themen und Fragen». Dabei lassen sich mit Biermann et al. (2014) zwei Aspekte besonders betonen: (1.) «die Chance zur Teilnahme für alle potenziell betroffenen Bürger» sowie (2.) «der 
argumentative Austausch, um eine öffentliche Meinung hervorbringen zu können». Da Habermas (1992) noch einer klassischen «massenmedial konstituierte[n] Öffentlichkeit und öffentlichen Meinung» gegenüberstand, ist für Biermann et al. (2014) die Frage, wie neue technologische Infrastrukturen wie soziale Medien neue Chancen für ideale «Diskursbedingungen» und «rationale Entscheidungsfindungen» neu zu verhandeln.

Der Partizipationsbegriff von Habermas ist für die Medienpädagogik auch insofern von Bedeutung, dass das Konzept zur Medienkompetenz von Baacke (1973) im Anschluss an die von Habermas formulierte kommunikative Kompetenz entwickelt wurde, d.h. im Anschluss an das Vermögen, an einem öffentlichen Diskurs überhaupt teilnehmen zu können. Für Biermann et al. (2014) ist diese Kompetenz nach dem Verständnis von Baacke (1973) «eng mit dem Partizipationsgedanken verknüpft», denn es gehe «letztlich um die Förderung von Partizipationskompetenz». Weiterführend ist das Konzept von Jenkins et al. (2009), welches bereits in Anlehnung an Moser (2010b) benannt wurde. Das Konzept von Jenkins et al. (2009) geht für Biermann et al. (2014) insofern über die Überlegungen von Baacke (1973) hinaus, da es nicht mehr nur darum geht «mit Medienangeboten kompetent umzugehen, sondern sich in den medialen Kulturräumen sicher und kompetent zu bewegen» (Baacke 1973, 9).

Trotz dieser unterschiedlichen Perspektiven und Begriffsreichweiten scheint Konsens darüber zu bestehen, dass soziale Medien weder technikdeterministisch zu einer aktiven Beteiligung an Lehr- und Lernaufgaben mit sozialen Medien führen noch einen rational geführten öffentlichen Diskurs von gesellschaftlich relevanten Themen etabliert haben. Dies wurde für den institutionellen Bildungskontext bereits in den Ausführungen zu den Spannungsfeldern in der Hochschullehre skizziert (Kap. 4.4.3). Insbesondere für den Bereich der Hochschullehre werden Phänomene ausbleibender Partizipation im Sinne ausbleibender Beteiligung in den Literaturberichten von Grell und Rau (2011) sowie Sim und Hew (2010) berichtet. Über die institutionelle Verwendung von sozialen Medien hinaus ist mit verschiedenen Mediennutzungsstudien belegbar (z. B. Busemannn und Gscheidle 2012; Busemannn 2013), dass soziale Medien von jüngeren Generationen, wie es Mayrberger und Moser (2011) formulieren, «erst zögerlich und teilweise gar rückläufig für die Artikulation eigener Interessen eingesetzt werden». Ferner weisen Mayrberger und Moser (2011) darauf hin, dass die These einer «(Netzgeneration», welche das Web 2.0 auf eine souveräne Art und Weise benutzt und die sich bietenden Partizipationschancen ausnutzt, um eigene Interessen zu artikulieren», empirisch nicht bestätigt werden konnte. Dies zeigt sich auch in der Beantwortung der Frage «Gibt es eine «net generation)?» von Schulmeister (2009a). Vielmehr beschränkt sich die Nutzung von sozialen Medien vor allem auf Webanwendungen und Apps zur Knüpfung und Pflege von Kontakten, welche insbesondere zur Kommunikation und zum Austausch verwendet werden. 
In Betrachtung weiterer Nutzungsstudien zeigt sich zudem, dass auch für ältere Generationen die Kommunikation im Fokus bei der Verwendung von Webanwendungen steht (z. B. Peterhans und Sagl 2011; Busemannn 2013). Über den deutschsprachigen Kontext hinaus zeigen White und Selwyn (2012) zudem, dass sich zwischen 2002 und 2010 die Internetnutzung im Allgemeinen sowie die Nutzung des Internets zum Lernen von Erwachsenen nicht grundlegend verändert hat. Auf Basis ihrer Daten kommen White und Selwyn (2012) zu der Aussage, dass die Verwendung vom Internet als Raum zum Lernen u. a. abhängig vom sozioökonomischen Hintergrund ist: «it is clear that educational» use of the internet is structured by age, occupational class and, to some extent, educational background» (White und Selwyn 2012). Allein die technische «zur Verfügungstellung» von neuen Potenzialen wird nur von einer kleinen Gruppe von Menschen genutzt. Mayrberger und Moser (2011) betonen vor diesem Hintergrund folgende Fragen aus medienpädagogische Perspektive:

«Aus medienpädagogischer Sicht stellen sich besonders Fragen nach dem spezifischen Partizipationspotenzial des Internets für unterschiedliche Zielgruppen wie Kinder, Jugendliche, Erwachsene oder Senioren und in welchen informellen und formalen Bildungskontexten eine Beteiligung in welchem Maße ermöglicht wird bzw. ermöglicht werden kann. Dabei sind auch die Motive für die Nutzung, vor allem aber Nicht-Nutzung von eingeräumten Beteiligungsmöglichkeiten in bildungsbezogenen Kontexten von Interesse» (Mayrberger und Moser 2011, o. S.).

Die von Mayrberger und Moser (2011) aufgeworfenen Themen und Fragen dienen als relevante Orientierung und Begründung für die eingangs formulierte Forschungsfrage, inwiefern die Mitgestaltung eines öffentlichen Wikibooks im Kontext eines Seminars der erziehungswissenschaftlichen Studienanteile des Lehramtsstudiums Lern- und Reflexionsanlässe zur Teilhabe an partizipativen Medienkulturen eröffnen kann.

\subsubsection{Reflexion von Privatheit und informationeller Selbstbestimmung}

Die Auseinandersetzung mit sozialen Medien eröffnet potenziell einen Zugang zu einem sich verändernden Verständnis von Privatheit und informationeller Selbstbestimmung sowie Reflexionspotenzialen im Umgang mit diesen Veränderungen. Dieses zweite Zukunftsargument zielt auf gesellschaftliche Mündigkeit in einer digital geprägten Kultur. Der potenzielle Zugang zu diesem sich verändernden Verständnis basiert zunächst auf der Beschreibung von Taddicken und Schmidt (2017, 5), dass sich - allgemein formuliert - unsere Welt verändert. Diese Veränderung wird mit (Taddicken und Schmidt 2017, 5) u. a. daran sichtbar, dass soziale Medien Einfluss 
auf die «Strukturen gesellschaftlicher Öffentlichkeit» nehmen. Dieser Einfluss zeigt sich für Taddicken und Schmidt $(2017,5)$ u. a. daran, dass soziale Medien mit der «Dominanz des Modus (Massenkommunikation) und seiner institutionalisierten Formen [brechen], indem sie andere Modi, Arenen und Kontexte bereitstellen, die Kommunikationsinhalte für einen (potenziell) großen Personenkreis sichtbar und zugänglich machen» (Taddicken und Schmidt 2017, 5).

Dies hat Konsequenzen für vielfältige Lebens- und Arbeitsbereiche. Ein relevanter Aspekt für Taddicken und Schmidt $(2017,5)$ ist in diesem Kontext das «Verständnis von Privatsphäre und informationeller Selbstbestimmung». So fällt die «Kontrolle über und die Abgrenzung von Publika für persönliche Informationen teils gewollt, oft aber ungewollt schwerer» (Taddicken und Schmidt 2017, 5). Das Thema von Kontrolle über persönliche Informationen im Umgang mit sozialen Medien wurde vor einigen Jahren vor allem im Kontext von Kampagnen wie «Think before you post» diskutiert. Im Fokus stand das Handeln von Jugendlichen und ihr Umgang mit persönlichen Informationen und Fotos am Beispiel von Webanwendungen zur Knüpfung und Pflege von Kontakten wie Facebook und WhatsApp. Spätestens seit dem Beginn der Big-Data-Debatte und der öffentlichen Auseinandersetzung mit psychometrischen Modellen, wird die Tragweite dieser (sich fortwährenden) Veränderungen auch für weitere Altersgruppen erfahrbar ${ }^{125}$. So wurde nicht zuletzt der - zumindest aus deutscher Perspektive scheinbar überraschende - Wahlerfolg von Trump mit der Möglichkeit zur Analyse von Social Media Profilen in Verbindung gebracht.

Die Schwierigkeit im Umgang mit der Kontrolle resultiert nach Taddicken und Schmidt $(2017,5)$ zum einen aus dem Charakter sozialer Medien. So sind Inhalte von bzw. Interaktionen mit sozialen Medien in der Regel (teil-)öffentlich. Es lässt sich daher einerseits die Frage stellen, wie andere Menschen mit den zugänglichen Daten und Informationen umgehen. Zum anderen betrifft die (Teil-)Veröffentlichung von Inhalten und Interaktionen auch die Frage nach Nutzungsrechten. So kann mit Kraut (2012 278) beispielsweise gefragt werden: «Was passiert mit eigenen Inhalten, die man beim jeweiligen Dienst einstellt? Darf der Betreiber diese verwenden, sie ohne Ankündigung löschen oder sie gar verkaufen?». Zum anderen resultiert die Schwierigkeit aus der, wie es Taddicken und Schmidt $(2017,5)$ formulieren, «Nicht-Flüchtigkeit» digitaler Informationen. Wie bereits im Kapitel 4.4.2.2 skizziert, ermöglicht das Teilen digitaler Inhalte - in Form von Fotos bis zu schriftlichen Kommentaren - einerseits die Artikulation eigener Ideen und Gedanken und anderseits auf diese Artikulationen anderer zu reagieren, diese zu thematisieren und zu referenzieren. So lassen sich Bildungspotenziale entsprechender Medien strukturell begründen. Die Möglichkeiten auf digital fixierte Gedanken Bezug nehmen zu können, bieten zugleich die

125 Ein Beispiel für den öffentlichen Diskurs über diese Thematik bietet dieser Beitrag: https://www. dasmagazin.ch/2016/12/03/ich-habe-nur-gezeigt-dass-es-die-bombe-gibt/ (29.01.2019). 
Möglichkeit zur algorithmischen Datenauswertung im Sinne von Big-Data- Aggregationen und -Analysen.

Neben diesen Herausforderungen auf technischer Ebene lassen sich weitere Herausforderungen auf unterschiedlichen Ebenen markieren. In diesem Kontext hat $u$. a. Kraut (2012) darauf hingewiesen, dass mit der Verwendung des Werkzeug- oder Toolbegriffs diese Ebenen ausgeblendet werden. Da für Kraut $(2012,278)$ «die meisten 〈Tools in Wirklichkeit Dienstleistungen eines Unternehmens sind und meist komplexe AGB und Datenschutzbestimmungen mit sich bringen», plädiert er einerseits für ein kritisches Hinterfragen des bisher verwendeten Toolbegriffs. Andererseits sensibilisiert er für verschiedene (häufig implizite) Entscheidungen und Konsequenzen, die mit der Verwendung sozialer Medien (in und für Bildungskontexte) einhergehen:

- Finanzierung: «Viele der Angebote sind in einer Basisversion kostenlos verfügbar, bieten allerdings auch kostenpflichtige Premiumdienste an. Können diese die laufenden Kosten für die vielen nicht zahlenden Nutzer wirklich decken? Wenn nein, wie finanziert sich der Dienst? Werbung? Nutzeranalyse?» (Kraut 2012, 278).

- Inhaltliche Kontrolle fremder Inhalte: «Wer erstellt sie und sind sie wirklich verlässlich bzw. vertrauenswürdig?»(ebd.).

- Verfügbarkeit: «In der heutigen Zeit geht man meist von ständiger Verfügbarkeit der Dienste aus, doch selbst größere Anbieter haben durchaus Probleme diese zu gewährleisten [3]. Und was ist, wenn der Betreiber aufgekauft wird, bankrott geht oder schlicht seinen kostenfreien Zugang einstellt?» (ebd.).

Entsprechende Fragen richten sich aus der Perspektive von Kraut $(2012,278)$ vor allem an «Webanwendungen und Apps zur Knüpfung und Pflege von Kontakten» sowie "Sharing-Gemeinschaften auf Basis gemeinsamer Interessen». So finanzieren sich soziale Medien wie Facebook oder Google über (zum Teil nicht von den Nutzerinnen und Nutzern intendierten) nutzer-generierten Daten ${ }^{126}$. So lässt sich mit Dander $(2014,3)$ auch grundsätzlich die Frage formulieren, wie (informationell) selbstbestimmt mit sozialen Medien umgegangen werden kann, wenn «den Anbieter[n] von Geräten und Plattformen gleichermaßen Zugriff auf die je eigenen Daten zu gewähren [ist]?».

Die Antwort von Dander $(2014,3)$ fällt ernüchternd aus: «Es scheint schier unmöglich, sich von diesem Zugriff zu emanzipieren, ohne gänzlich auf denkbare positive Effekte der Datennutzung zu verzichten» (Dander 2014, 3). Zudem lassen sich nach Dander (2014) Möglichkeiten skizzieren, wie Auseinandersetzung mit sozialen Medien einen Zugang zu einem bewussten Umgang mit Daten und damit auch einem sich verändernden Verständnis von Privatheit und informationeller Selbstbestimmung

126 Einen unterhaltsamen Einblick in die Praktiken und Funktionsweisen zur Sammlung und Analyse von Nutzerinnen- und Nutzerdaten eröffnet diese Doku-Reihe: https://donottrack-doc.com/de/ (29.01.2019). 
eröffnen. Wenngleich die Arbeit mit den Daten der Lernenden hinsichtlich ihrer eigenen Privatsphäre von Dander $(2014,6)$ als nicht unproblematisch dargestellt wird, kann sie «zumindest für diese selbst eine Konfrontation mit den digitalen Speicherungspraktiken von staatlichen und unternehmerischen Akteuren erfahrbar machen». Dafür verweist Dander (2014) auf die Vorgehensweisen von Spitz (2014), um auf die über einen gespeicherten persönlichen Daten auf Grundlage des deutschen Bundesdatenschutzgesetzes (BDSG) von unterschiedlichen Webunternehmen Zugang zu erhalten.

«Auf diese Weise kann nicht nur nachvollzogen werden, welche personenbezogenen Daten in welchen Mengen und in welcher Form gespeichert werden; die gesetzliche Grundlage der Aufforderung zur Datenherausgabe verbindet diese Erfahrungen direkt mit BürgerInnenrechten und kann somit zu einer Reflexion über die Bedeutung des Datenschutzes und der Privatsphäre für demokratisch verfasste Staaten führen» (Dander 2014, 6).

Während dieses Vorgehen vor allem für soziale Medien im Sinne von Webanwendungen und Apps zur Knüpfung und Pflege von Kontakten sowie für Sharing-Gemeinschaften auf Basis gemeinsamer Interessen Nutzerinnen und Nutzer sensibilisieren kann, bietet auch die Arbeit mit sozialen Medien im Sinne von hochschulinternen Lernplattformen, das Potenzial für entsprechende Erfahrungen. «Learning Analytics» heisst in diesem Kontext das Schlagwort (oder der Fachbegriff), der das Sammeln von Daten über Lernende, um diese zu unterstützen, bezeichnet (Kap. 3.4.2.2). Aus der Perspektive von Dander $(2014,7)$ bieten sich diese Daten auch an, um «in anonymisierter Form zum Gegenstand von gemeinsamen Analysen, Interpretationen, Diskussionen und Kritik gemacht zu werden». Im Zusammenhang mit Wikis ist dabei interessant, dass die jeweils gespeicherten Informationen über die Versionsgeschichte in transparenter Weise offengelegt werden und zum Thema gemacht werden können.

\subsubsection{Zwischenfazit zum Lernen über soziale Medien}

In Anknüpfung an die vorgestellten medienerzieherischen Überlegungen können die zwei Teilkapitel «Teilnahme und Teilhabe an einer partizipativen Kultur» (Kap. 4.5.2.1) und «Reflexion von Privatheit und informationeller Selbstbestimmung» (Kap. 4.5.2.2) als Antworten auf die Fragestellungen verstanden werden, für welche aktuellen Themen und Fragen Lehramtsstudierende in der Auseinandersetzung mit sozialen Medien sensibilisiert werden können (und sollten). Diese Antworten können als Zukunftsargumente nach Döbeli Honegger (2016) ${ }^{127}$ verstanden werden und zielen in

127 Einschränkend muss an dieser Stelle angemerkt werden, dass Döbeli Honegger (2016) in der Diskussion der Frage, warum das Digitale in die Schule gehört, verschiedene Zukunftsargumente formulieren. Die Zukunftsargumente «Employability» sowie «Lebensweltargumente» stehen in der vorliegenden Arbeit nicht im Fokus. 
insbesondere auf gesellschaftliche Teilhabe bzw. sozial verantwortliches Handeln in einer digital geprägten Kultur. Diese Themenstellungen sind insbesondere vor dem Hintergrund der Überlegungen zur medienpädagogischen Professionalisierung von Lehramtsstudierenden relevant (Kap. 4.3.2.4) und werden im Rahmen des Entwurfs erneut aufgegriffen (Kap. 6.6).

\subsection{Zusammenfassung}

Mit den vorgestellten Überlegungen dieses Kapitels wurde ein zentraler Ausgangspunkt des entwicklungsorientierten Bildungsforschungsprojektes vorgestellt. Im Fokus stand die Identifikation praxisbezogener Problemstellungen sowie die Auseinandersetzung mit wissenschaftlichen Perspektiven zur integrativen Medienbildung in der Lehrerinnen- und Lehrerbildung. Bei Betrachtung bildungspolitischer Leitbildern zur (digitalen) Medienbildung für die Schule sowie der aktuellen Lehrerinnen- und Lehrerbildung in der Universität zeigt sich eine deutliche Diskrepanz. Während von Lehrkräften einerseits erwartet wird, dass sie in einem integrativen Ansatz in ihren jeweiligen Fächern zur Medienkompetenzentwicklung ihrer Schülerinnen und Schüler beitragen sollen, eröffnet das Lehramtsstudium dafür andererseits kaum adäquate Erfahrungsmöglichkeiten und Reflexionsanlässe (Kap. 4.2). Zudem konnten theoretische und empirische Grundlagen zur Gestaltung sowie zur Analyse eines Lernens mit und über soziale Medien in der Lehrerinnen- und Lehrerbildung vorgestellt werden (Kap. 4.3, 4.4, 4.5).

Zu Beginn des Kapitels erfolgte eine methodische Verortung des Kapitels in der Logik einer entwicklungsorientierten Bildungsforschung (Kap. 4.1). Als zentraler Bestandteil der von Sesink und Reinmann (2015) geforderten Phase der Problematisierung erfolgte eine Diskussion aktueller bildungspolitischer Leitbilder (Kap. 4.2.1). Als zentrale Zielvorstellungen wurden drei Elemente herausgearbeitet:

1. Medienbildung im Sinne einer institutionellen Vermittlung von Medienkompetenz wird als allgemeiner Bildungs- und Erziehungsauftrag markiert (Kap. 4.2.1.1).

2. Statt in Form eines eigenen Lernbereichs bzw. Unterrichtsfachs soll Medienbildung in Schulen in einem integrativen Ansatz erfolgen (Kap. 4.2.1.3).

3. Ein zentrales Element integrativer Medienbildung ist das Lernen mit und über den innovativen Einsatz (digitaler) Medien (Kap. 4.2.1.2).

Beim Vergleich dieser Zielvorstellungen und Erwartungen an aktuelle Lehrkräfte mit der aktuellen Situation der universitären Lehrerinnen- und Lehrerbildung wurde eine deutliche Diskrepanz markiert (Kap. 4.2.1.4). So finden sich auf bildungspolitischer Ebene weder angemessene Leitbilder für die Lehrerinnen- und Lehrerbildung für diesen Bereich noch eine angemessene Praxis (Kap. 4.2.1.4). An den zwei Problemdimensionen wurden Herausforderungen der Praxis der TU Darmstadt 
verdeutlicht (Kap. 4.2.2.1, 4.2.2.2). Zur Identifikation möglicher Handlungsoptionen wurde die Idee skizziert, Seminare in den erziehungswissenschaftlichen Studien für Lehramtsstudierende zu konzipieren und wissenschaftlich zu untersuchen.

Im Kapitel 4.3 erfolgte eine Konkretisierung von und Positionierung zu den Begriffen und Konstrukten (1.) Medienbildung und Medienkompetenz, (2.) medienpädagogische Kompetenz sowie (3.) soziale Medien. Der Begriff der Medienbildung wird im Rahmen der Arbeit nach Tulodziecki et al. $(2010,178)$ «als zusammenfassender Begriff für alle bildungsrelevanten Prozesse mit Medienbezug mit der Zielstellung eines bestimmten Kompetenzniveaus» verstanden. Zur Beschreibung von Zielen spezifischer Lehraktivitäten und Ergebnisse individueller Lernprozesse wird in Anlehnung Jörissen (2011), Tulodziecki et al. (2010) und Mayrberger (2012a) der Medienkompetenzbegriff verwendet (Kap. 4.3.1). Nach Tulodziecki $(1997,116)$ wird Medienkompetenz verstanden als «Fähigkeit und Bereitschaft zu einem sachgerechten, selbstbestimmten, kreativen und sozial verantwortlichen Handeln in einer von Medien mitgestalteten Welt». Mit dem Begriff «medienpädagogische Kompetenz» wird die Fähigkeit, das Wissen sowie die Handlungsbereitschaft gefasst, medienpädagogischer und mediendidaktischer Lehr- und Lernaktivitäten planen und realisieren zu können. Für das vorliegende Teilprojekt wurde - neben der vorherigen Diskussion des Medienkompetenzbegriffs - insbesondere das Teilkonstrukt der mediendidaktischen Kompetenz diskutiert (Mayrberger 2012a; Blömeke 2000, 2005). In Anlehnung an Mayrberger (2012a) wurde entschieden, die Auseinandersetzung mit sozialen Medien zur Anregung von Lernprozessen zu fokussieren (Kap. 4.3.2). Der Terminus «soziale Medien» dient als «Sammelbegriff für Angebote auf Grundlage digital vernetzter Technologien, die es Menschen ermöglichen, Informationen aller Art zugänglich zu machen und davon ausgehend soziale Beziehungen zu knüpfen und/oder zu pflegen» (Taddicken und Schmidt 2017, 8). Ferner wurde zur Differenzierung der vorgestellten Arbeitsdefinition zwischen drei verschiedenen Typen von sozialen Medien unterschieden: (a) Webanwendungen und Apps zur Knüpfung und Pflege von Kontakten, (b) hochschulinterne Lernplattformen und (c) Sharing-Gemeinschaften auf Basis gemeinsamer Interessen (Kap. 4.3.3).

In Kapitel 4.4 wurden mediendidaktische Erkenntnisse und Überlegungen zum Einsatz sozialer Medien in der universitären Lehr- und Lernsituationen vorgestellt. Dabei wurden sowohl theoretische Potenziale sozialer Medien zum Lernen skizziert (Kap. 4.4.2) sowie damit in Verbindung stehende Spannungsfelder in der Hochschullehre an empirischen Beispielen exemplarisch erläutert (Kap. 4.4.3). Die vorgestellten Überlegungen eröffneten zudem erste Verortungsmöglichkeiten innerhalb des mediendidaktischen Forschungsdiskurses. Auf Basis der vorgestellten Begriffsdifferenzierung wurde argumentiert, dass verschiedene lerntheoretische Potenziale von sozialen Medien nicht für alle Typen von sozialen Medien in gleichem Masse gelten. Wenngleich hochschulinterne Lernplattformen beispielsweise ebenfalls Funktionalitäten 
wie Blogs oder Wikis bereitstellen und damit Funktionalitäten von öffentlichen Sharing-Gemeinschaften adaptieren, sind sie nicht mit diesen zu verwechseln. Wenn in der Diskussion über die Möglichkeiten von sozialen Medien beispielsweise die Praktiken des Sharings (Kap. 4.4.2.2) als Blick über den institutionellen Tellerrand bzw. zur Identifikation interessanter Menschen markiert werden, wird damit auch eine zugehörige Sharing-Gemeinschaft vorausgesetzt. Innerhalb hochschulinterner Plattformen stehen entsprechende Optionen nicht zu Verfügung. Bei der Diskussion konkreter Gestaltungsmöglichkeiten und aktueller Spannungsfelder einer interaktiven und kollaborativen Hochschullehre mit sozialen Medien wurden exemplarische Szenarien mit (vorwiegend hochschulinternen) Blogs als Reflexionsinstrumente sowie Wikis als Feedbackumgebungen skizziert. Dabei wurden zum einen unterschiedliche Strategien zur Anregung von Austausch- und Kollaborationsprozessen sichtbar. Zum anderen wurden Spannungsfelder bei Bewertungssituationen kollaborativer Lernformate, im Umgang mit neuen Inhaltsformen digitaler Produkte und dem Anspruch an eine Feedbackkultur im Kontext von Massenveranstaltungen aufgezeigt. Diese Herausforderung können u. a. auf die Diskrepanz zwischen den skizzierten Lernverständnissen einerseits und der Organisationslogik formaler Bildungsinstitutionen andererseits zurückgeführt werden (Dohn 2009). Mit Blick auf das skizzierte Leitbild des Wandels vom Lehren zum Lernen können für die Praxis der Hochschullehre zwei Aspekte festgehalten werden: (1.) Der Einsatz digitaler sozialer Medien kann grundsätzliche Spannungsfelder der Hochschullehre nicht auflösen. (2.) Unter der Anerkennung der gegebenen Rahmenbedingungen lassen sich die durch soziale Medien eröffnenden Potenziale in Ansätzen realisieren (Kap. 4.4.3).

In Anlehnung an die Argumentation von Petko (2011) wird davon ausgegangen, dass gestaltungsorientierte Forschungsansätze die Möglichkeit eröffnen, medienpädagogische Perspektiven und mediendidaktische Forschung in der deutschsprachigen Debatte näher zusammenzubringen: «Es ginge darum herauszufinden, wie Menschen mit Medien für Medien sensibilisiert werden können» (Petko 2011, 248). Daran anknüpfend widmete sich das Kapitel «Soziale Medien als Gegenstand universitärer Medienbildung» der Frage, wie Menschen für (soziale) Medien sensibilisiert werden können und welche Themen in Auseinandersetzung mit digitalen sozialen Medien als relevant erscheinen. Eine Sensibilisierung für Medien wurde als Vermittlung von Medienkompetenz konkretisiert. Aus medienerzieherischer Perspektive wurden handlungsorientierte Ansätze und Gestaltungsprinzipien zur Förderung von Medienkompetenz präsentiert (Kap. 4.5.1). Bei Betrachtung des aktuellen medienpädagogischen Fachdiskurses wurden zudem zwei relevante Themenstellungen im Kontext digitaler sozialer Medien erarbeitet: Teilnahme und Teilhabe an einer partizipativen Kultur (Kap. 4.5.2.1) sowie Reflexion von Privatheit und informationeller Selbstbestimmung (Kap. 4.5.2.1). 
Die vorgestellten theoretischen und empirischen Erkenntnisse in Form mediendidaktischer und medienerzieherischer Überlegungen erfüllen mehrere Funktionen. Zunächst konnte eine praxisbezogene Problemstellung und eine zugehörige Gestaltungsidee herausgearbeitet werden. Die Kapitel zur begrifflich-theoretischen Positionierung sowie die vorgestellten mediendidaktischen und medienerzieherischen Überlegungen dienen im Folgenden als grundlegende Orientierungspunkte für die theoriegeleitete Entwicklung eines Konzepts sowie als Entwurf einer Lehrveranstaltung (Kap. 6). Zudem bieten sie Anschlussstellen an aktuelle mediendidaktische und medienpädagogische Forschungsdiskurse und werden als Begründung für bestimmte Forschungsansätze herangezogen (Kap. 3, 7, 11). 


\section{Erfahrung-Theorie-Relationierung mit Metaphern in der Pädagogik}

\subsection{Methodische Verortung}

Mit dem vorliegenden Kapitel wird ein weiterer zentraler Ausgangspunkt des durchgeführten Forschungs- und Praxisprojektes vorgestellt und diskutiert (Abb. 5.1). In Anknüpfung an das vorherige Kapitel wird die Problematisierung der Praxis um eine Perspektive erweitert. Im Fokus stehen aktuelle Leitbilder und Forschungsergebnisse über die Praxis der erziehungswissenschaftlichen Studienanteile im Lehramtsstudium (Kap. 5.2). Es werden die normativen Orientierungen bildungspolitischer Leitbilder zur Bedeutung und Funktion des Lehramtsstudiums im Rahmen der Lehrerinnen- und Lehrerbildung skizziert (Kap. 5.2.1), deren Passung zur Studienordnung der TU Darmstadt diskutiert und den empirischen Forschungsergebnissen zu erfahrungsfundierten Vorstellungen als Lernvoraussetzungen von Lehramtsstudierenden gegenübergestellt (Kap. 5.2.2). Zum Umgang mit erfahrungsfundierten Vorstellungen werden Überlegungen zur reflexiven Lehrerinnen- und Lehrerbildung markiert.

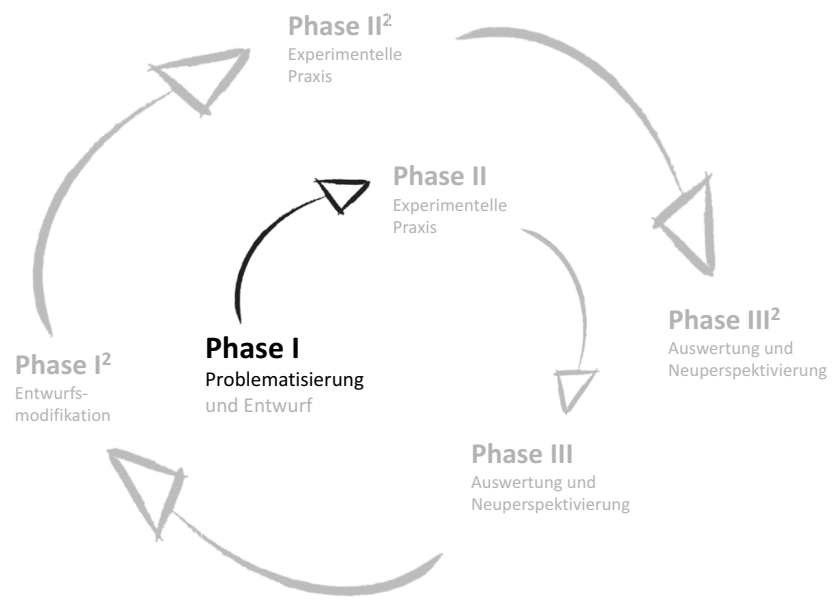

Abb. 5.1: Verortung des Kapitels im Phasenmodell der entwicklungsorientierten Bildungsforschung.

In Adaption der von Sesink und Reinmann (2015) skizzierten Forschungsphase «Problematisierung und Entwurf» erfolgt, analog zu Kapitel 4, eine Erarbeitung des aktuellen Forschungsstandes. Die Auseinandersetzung dient der Vorbereitung eines theoretisch begründeten Entwurfs. In diesem Kontext werden die Konstrukte «Beliefs», «Subjektive Theorien» und «metaphorische Konzepte» vergleichend diskutiert. Es erfolgt eine begriffliche und theoretische Verortung sowie eine Diskussion über deren wissenschaftliche Bedeutung und deren praktische Relevanz für das Projekt (Kap. 5.3). Auf Basis der begrifflich-theoretischen Diskussion wurde entschieden, die 
Auseinandersetzung mit Metaphern zur Gestaltung der Praxis sowie als Forschungsgegenstand und -instrument zu fokussieren. Mit Blick auf den deutschsprachigen Diskurs werden exemplarisch die Überlegungen von Gropengießer (2004) zu Denkfiguren des Lehrens und Lernens vorgestellt (Kap. 5.4.1). Um einen Einblick in empirische Arbeiten zur Verwendung von Metaphern in der Lehrerinnen- und Lehrerbildung zu eröffnen, werden über den deutschsprachigen Diskurs hinaus insbesondere englischsprachige Publikationen im Bereich «teacher education» berücksichtigt (Kap. 5.4). Abschließend werden ausgewählte Diskurse und Erkenntnisse zur Auseinandersetzung mit Metaphern in der Erziehungswissenschaft präsentiert (Kap. 5.5). Die Auswahl umfasst Arbeiten zum metaphorischen Gehalt der pädagogischen Fach- und Alltagssprache (Kap. 5.5.1), weitere - aus forschungsmethodischer Sicht interessante - empirische Projekte (Kap. 5.5.2) sowie didaktische Funktionen von Metaphern (Kap. 5.5.3).

Mit Verweis auf das vorherige Kapitel kann ebenfalls markiert werden, dass die vorgenommene Verknüpfung einer praxisorientierten Problematisierung sowie die Erarbeitung des aktuellen Forschungsstandes - wenngleich mit einem anderen thematischen Schwerpunkt - zwei Funktionen erfüllt. Die Berücksichtigung der zwei Prozess- und Qualitätsstandards «Praxisrelevanz von Forschungsfragen und Praxistauglichkeit von Lösungen» (Tulodziecki et al. 2013, 228) und «Zusammenwirken von Forschung und Praxis» (Tulodziecki et al. 2013, 229) konnte über einen temporären Perspektivwechsel realisiert werden.

\subsection{Problematisierung}

Die Problematisierung bzw. das Erkennen eines Problems umfasst für Sesink und Reinmann (2015) das Hinterfragen des Verhältnisses von realer Praxis und normativer Orientierung sowie der zwei zugehörigen Pole (Kap. 3.1.1). Dafür widmet sich das folgende Kapitel im ersten Schritt einer Betrachtung des Leitbildes «Lehrkräfte als Expertinnen und Experten für das Lehren und Lernen» (Kap. 5.2.1). Im zweiten Schritt erfolgt eine Diskussion der realen Praxis der erziehungswissenschaftlichen Studien im Lehramtsstudium (Kap. 5.2.2). Dies erfolgt durch die Betrachtung der Studienordnung der TU Darmstadt (Kap. 5.2.2.1) sowie unter Berücksichtigung von Erkenntnissen über die Lernvoraussetzungen von Lehramtsstudierenden (Kap. 5.2.2.2). Handlungsstrategien zum Umgang mit dem Spannungsfeld zwischen normativen Zielsetzungen des Lehramtsstudiums einerseits und den nicht passgenauen erfahrungsfundierten Vorstellungen der Lehramtsstudierenden anderseits werden zum Abschluss des Kapitels diskutiert (Kap. 5.2.3). 


\subsubsection{Lehrkräfte als Expertinnen und Experten für das Lehren und Lernen}

Die Formulierung des Berufsleitbildes «Lehrkräfte als Expertinnen und Experten für Lehren und Lernen» diente der von der Kultusministerkonferenz eingesetzten Kommission als Ausgangspunkt, um «Eckpunkte für die Ausgestaltung der Lehrerbildung» (Terhart 2000, 46) zu markieren. An diesen Expertenbericht anknüpfend wurde in der «Bremer Erklärung» die Kernaufgabe von Lehrerinnen und Lehrern als «gezielte und nach wissenschaftlichen Erkenntnissen gestaltete Planung, Organisation und Reflexion von Lehr- und Lernprozessen sowie ihre individuelle Bewertung und systemische Evaluation» (KMK 2000, 2) formuliert. Diese Erklärung dient auch in aktuellen Beschlüssen der KMK (2014, 2016b) als Referenzpunkt des Berufsbildes von Lehrerinnen- und Lehrern und damit als Basis zur Formulierung von Anforderungen und Perspektiven für die Lehrerinnen- und Lehrerbildung im Allgemeinen (Terhart 2000) sowie für die bildungswissenschaftlichen Bestandteile im Besonderen (KMK 2014; Horstkemper 2004).

Die zentrale Aufgabe von Lehrerinnen und Lehrern wird konsensual in der professionellen Gestaltung von Lehren und Lernen gesehen (z. B. KMK 2000; Horstkemper 2004; Terhart 2000). Eine nähere Bestimmung dieses zentralen Aufgabenbereiches erfolgt in den verschiedenen Publikationen und Beschlüssen jedoch in einem unterschiedlichen Umfang und mit leicht unterschiedlichen Akzentuierungen. Horstkemper $(2004,463)$ macht beispielsweise deutlich, dass «die Fähigkeit zur Organisation von Lehren und Lernen» als zentrale Kompetenz von Lehrerinnen und Lehrern betrachtet werden kann. Diese Fähigkeit umfasst, so Horstkemper (2004, 463), «nicht nur die Vermittlung von Wissen, Fähigkeiten und Wertorientierungen, sondern schließt ebenso die Gestaltung von Lernumgebungen [ein]». Weitere zugehörige Aspekte sind für Horstkemper $(2004,463)$ u. a. die «Entwicklung von Schulleben und Schulkultur» sowie die «Gestaltung des eigenen Arbeitsplatzes». Daran anknüpfend benennt Horstkemper $(2004,463)$ verschiedene Kompetenzen über die Lehrerinnen und Lehrer verfügen müssen, um dieser Aufgabe zu genügen. In den aktuellen «Standards für die Lehrerbildung: Bildungswissenschaften» wird von der KMK (2014) auf den «Bremer Beschluss» (KMK 2000) verwiesen. Lehrkräfte werden als «Fachleute für das Lehren und Lernen» verstanden ${ }^{128}$. Auf Basis dieses Leitbildes werden von der KMK (2014) vier Kompetenzbereiche für die Bildungswissenschaften differenziert, um die notwendigen Fähigkeiten und Kompetenzen von Lehrerinnen und Lehrern präziser zu bestimmen. Dazu zählen die Kompetenzbereiche: «Unterrichten» (KMK 2014, 7), «Erziehen» (ebd., 9), «Beurteilen» (ebd., 11.) und «Innovieren» (ebd., 13). Deutlich ausführlicher wird die übergeordnete Aufgabe von Lehrerinnen und Lehrern im Abschlussbericht der Kommission der KMK gefasst (Terhart 2000, 48 f.):

128 Wenngleich die Formulierung der Kernaufgabe von Lehrerinnen und Lehrern in den zwei Beschlüssen der Kultusministerkonferenz übereinstimmen (KMK 2014, 2000), heisst es im «Bremer Beschluss «Lehrerinnen und Lehrer sind Fachleute für das Lernen» (KMK 2000, 2). Inwiefern es sich bei der Erweiterung des Begriffs des «Lehren» um eine Aktualisierung des Berufsbildes von Lehrerinnen und Lehrern handelt, wird im aktuellen Beschluss der KMK (2014) nicht konkretisiert. 
«Die Kommission betrachtet die gezielte Planung, Organisation, Gestaltung und Reflexion von Lehr-Lern-Prozessen als Kernbereich der Kompetenz von Lehrerinnen und Lehrern» (Terhart 2000, 48).

Zur weiteren Präzisierung erfolgt $u$. a. eine Abgrenzung vom Alltagsverständnis von Lehren, welches sich auf die Vermittlung von Fachwissen beschränkt. Lehren umfasst im Sinne der Kommission (Terhart 2000, 48) «die Unterstützung von z. B. fächerübergreifendem, problembezogenem und kooperativem Lernen» sowie das «Anregen und Fördern von selbstorganisiertem Lernen» (ebd.). Darüber hinaus zielt Lehren gemäß der Kommission (Terhart 2000, 48) auf den Aufbau sozialer Kompetenzen, motivationalen Orientierungen und entsprechenden Einstellungen». Wie bei den zuvor markierten Positionen (KMK 2014; Horstkemper 2004) werden auch von der Kommission der KMK (Terhart 2000, 48 ff.) «vielfältige Teilkompetenzen» formuliert, die für eine professionelle Erfüllung des übergeordneten Leitbildes erforderlich sind und im Rahmen der Lehrerinnen- und Lehrerbildung entwickelt werden sollen.

Der Begriff der Kompetenzen umfasst in diesem Kontext unterschiedliche Facetten. Die Kommission der KMK (Terhart 2000, 54) verwendet den Begriff der Kompetenz als «das Verfügen über Wissensbestände, Handlungsroutinen und Reflexionsformen, die aus Sicht einschlägiger Professionen und wissenschaftlicher Disziplinen zweck- und situationsangemessenes Handeln gestatten». Für die Lehrerinnen- und Lehrerbildung lässt sich die Frage formulieren, auf welcher Basis entsprechende Kompetenzen erworben bzw. aufgebaut werden können. Zur Beantwortung dieser Frage wird von Terhart $(2000,55)$ eine weitere Differenzierung vorgenommen, welche auch von Horstkemper (2004) aufgegriffen wird. Demnach lassen sich drei Grundlagen differenzieren:

- Wissenschaftlich fundiertes Wissen: Um relevante «Tätigkeiten und Funktionszusammenhänge» des Lehrerinnen- und Lehrerberufs zu erkennen, professionell bearbeiten und reflektieren zu können, bedarf es nach Terhart $(2000,55)$ «wissenschaftlich fundierte[s] Wissen». Dieses Wissen kann nach unterschiedlichen Kriterien differenziert werden und umfasst für Terhart $(2000,55)$ deklarative, prozedurale und metakognitive Komponenten. Dazu zählt ebenfalls Wissen aus den verschiedenen inhaltlichen Bereichen, wie beispielsweise «aus der Fachdidaktik» und «der Erziehungswissenschaft». Wissen kann im Kontext der Lehrerinnen- und Lehrerbildung als notwendiges, aber nicht als hinreichendes Kriterium professionellen Handelns von Lehrkräften betrachtet werden (Terhart 2000; Horstkemper 2004).

- Flexibel anwendbare Handlungsroutinen: Zur Bewältigung der komplexen Handlungssituationen im Unterricht, in denen Lehrkräfte in «Sekundenbruchteilen Lösungen für ein ständig im Fluss befindliches soziales, motivationales und kognitives Geschehen generieren» müssen, bedarf es aus der Sicht von Terhart (2000, 
55 f.) routinisierter Handlungsmuster. Die Aneignung sowie die reflektierte Modifikation entsprechender Muster und Routinen muss, so Terhart $(2000,56)$ «im beruflichen Handeln entwickelt werden».

- Berufsethos: «Der verantwortliche Umgang mit Heranwachsenden», setzt gemäß Terhart $(2000,56)$ eine pädagogische Haltung voraus, die das pädagogische Handeln als Lehrkraft leitet. Dazu zählt Terhart $(2000,56)$ u. a. "soziales Geschick, Einfühlungsvermögen, Gesprächsbereitschaft, Engagement, Empathie, Geduld und Zuversicht». Um den professionellen Anforderungen als Lehrkraft zu genügen, müssen Lehrerinnen und Lehrer nach Terhart $(2000,56)$ «sich zielgerichtet und bewusst ein pädagogisch vertretbares Berufsethos [...] erarbeiten».

Auf Basis des skizzierten Leitbildes sowie der verschiedenen Facetten des Kompetenzbegriffs soll das Lehramtsstudium nach Auffassung der Kommission der KMK gemäß Terhart $(2000,83)$ «die wissenschaftliche Basis für die Entwicklung der professionellen Kompetenzen von Lehrern vermitteln». Dafür differenziert Terhart (2000) zwischen vier Studienelementen der ersten Phase: Fachstudien (ebd., 99 ff.), fachdidaktische Studien (ebd., 102 ff.), erziehungswissenschaftliche Studien (ebd., 104 ff.) und schulpraktische Studien (ebd., 107 ff.). Diese verschiedenen Studienanteile tragen in je unterschiedlicher Weise dazu bei, die wissenschaftliche und theoretische Basis für die Entwicklung relevanter Teilkompetenzen bereitzustellen. Mit der Formulierung einer «berufsorientierten Wissenschaftlichkeit» markiert Terhart (2000, 83) eine Anforderung bzw. einen Anspruch an das Lehramtsstudium, welcher sich aus der Perspektive von Horstkemper $(2004,472)$ vor allem auf fachdidaktische und erziehungswissenschaftliche Anteile bezieht. So hat sich die Auseinandersetzung mit relevanten Themen, Theorien und wissenschaftlichen Methoden «sowohl an der Struktur der Disziplin zu orientieren als auch immer den Blick auf Verwendungsinteressen im beruflichen Handlungsfeld zu richten». Erziehungswissenschaftliche Studien bewegen sich in Anlehnung an Horstkemper $(2004,471)$ im «Spannungsfeld zwischen Wissenschafts- und Praxisbezug». Im Rahmen dieses Spannungsfeldes steht die Vermittlung von «Orientierungs- und Reflexionswissen» für Horstkemper (2004, 471) im Fokus:

«Es soll Orientierungs- und Reflexionswissen vermittelt werden, auf dessen Grundlage die eigenen Handlungsbedingungen theoriegeleitet analysiert und gestaltet werden können. Unmittelbare Handlungsfähigkeit zu erzeugen ist dabei noch nicht das Ziel. Dennoch soll aber die Wissensvermittlung mit Bezug auf das Berufsfeld vermittelt werden» (Horstkemper 2004, 471).

Die Fokussierung der Wissensvermittlung findet sich auch in den Standards für den Bereich der Bildungswissenschaften (KMK 2014). Zur Präzisierung der Kompetenzen hinsichtlich der Schwerpunktsetzungen für die erste Phase werden vorzugsweise 
wissensbezogene Operatoren verwendet, z. B. «kennen» (33x), «wissen» (10x) und «verfügen über Kenntnisse» ( $1 \mathrm{x})$. Operatoren für stärker reflexive oder handlungsorientierte Ansätze finden sich aber überraschend selten, z. B. «reflektieren» (7x), «analysieren» $(1 \mathrm{x})$ oder «bewerten» $(1 \mathrm{x})$. Diese Schwerpunktsetzung überrascht zum einen dahingehend, dass in den von der $\operatorname{KMK}(2014,6)$ vorgeschlagenen Ansätzen zur «Vermittlung bildungswissenschaftlicher Inhalte» keine rezeptionsorientierten Ansätze benannt werden. Zum anderen weisen u. a. Horstkemper (2004) und Terhart (2000) darauf hin, dass pädagogisch wissenschaftliches Wissen aufgrund der Komplexität der Handlungssituationen von Lehrerinnen und Lehrern nicht technologisierbar ist. Für Horstkemper $(2004,465)$ ist es entsprechend wichtig, «Theoriewissen nicht lediglich als ‘Vorratswissen` vor Eintritt in die berufliche Praxis anzuhäufen, es als «träges Wissen) abzulegen, das mehr oder weniger nützlich für die spätere Bewältigung beruflicher Aufgaben erscheint». Vielmehr kann Theoriewissen - verstanden als Reflexionswissen - dazu beitragen, die eigenen Erfahrungen, Handlungen und Handlungsbedingungen in pädagogischen Kontexten denkend verarbeiten und beurteilen zu können. In diesem Sinne markiert auch Terhart (2002, 21), dass neben der Entwicklung einer Wissensbasis im Lehramtsstudium auch die Entwicklung von Reflexionsfähigkeit entscheidend ist. Die Fähigkeit, «Theorie- und Praxiswissen aufeinander zu beziehen und dadurch eine reflexive Distanz zur eigenen Berufsarbeit herzustellen» bezeichnet Meyer $(2003,101)$ ebenfalls als Reflexionskompetenz.

Ansätze in der Lehrerinnen- und Lehrerbildung in denen die Entwicklung einer Reflexionsfähigkeit bzw. -kompetenz in besonderer Weise betont werden, werden u. a. unter dem Begriff der «reflexiven Lehrerbildung» diskutiert (Abels 2011; Stein 2007; Wildt 2003). Für Stein (2007) zeichnet sich eine reflexive Lehrerinnen- und Lehrerbildung dadurch aus, dass «eine zentrale Ausbildungsaufgabe in der Reflexion beruflichen Handelns» gesehen wird. Dafür betont Stein $(2007,4)$ die Suche «nach möglichen Dialogformen zwischen Hochschule und Schule». Im Zusammenhang mit diesem Fokus steht für Stein (2007) «eine Abkehr von der Sicht, dass richtiges Wissen über Unterricht nur von wissenschaftlichen Experten erzeugt und im Rahmen der Lehrerbildungsphasen vermittelt werden kann» (Stein 2007, 4). In der Diskussion verschiedener Ansätze einer reflexiven Lehrerinnen- und Lehrerbildung weist Abels $(2011,48)$ darauf hin, dass es einen Konsens darüber gibt «dass reflexive Fähigkeiten die berufliche Entwicklung von Lehrkräften in wünschenswerter Weise voranbringen könnten». Für Wildt (2003) besteht die Aufgabe der Lehrerinnen- und Lehrerbildung in einem angemessenen In-Beziehung-Setzen von Theorie und Praxis in Form von Reflexion bzw. reflexiven Lernens.

Die in Form des Berufsleitbildes «Lehrkräfte als Expertinnen und Experten für das Lehren und Lernen» vorgestellten normativen Orientierungen und Implikationen für das Lehramtsstudium bietet einen ersten Rahmen zur kriteriengeleiteten Betrachtung der Praxis der TU Darmstadt. Zentrale Ziele sind zum einen der Aufbau einer 
pädagogischen Wissensbasis. Zum anderen wird die Entwicklung einer Reflexionskompetenz bzw. -fähigkeit als zentrales Ziel markiert. Das Verhältnis zwischen diesen zwei Zielstellung wird unterschiedlich thematisiert.

\subsubsection{Praxis der erziehungswissenschaftlichen Studien im Lehramtsstudium}

Die folgenden Ausführungen gliedern sich in zwei Teile. Im ersten Schritt werden die zentralen Ziele der Studienordnung der Grundwissenschaften im Lehramtsstudium skizziert (Kap. 5.2.2.1). Im Vergleich mit den Zielen des vorgestellten Leitbildes «Lehrkräfte als Expertinnen und Experten für das Lehren und Lernen» zeigen sich vielfältige Anknüpfungspunkte und Übereinstimmungen zur Praxis der TU Darmstadt auf der Ebene der Studienordnungen in den Grundwissenschaften. Im zweiten Schritt werden empirische Befunde zu erfahrungsbasierten Vorstellungen und Lernvoraussetzungen von Lehramtsstudierenden vorgestellt (Kap. 5.2.2.2). Es zeigt sich ein spannungsvolles Verhältnis zu den formulierten Zielstellungen. Dieses Spannungsverhältnis wird als zentrale Herausforderung markiert.

\subsubsection{Ziele der Grundwissenschaften der TU Darmstadt}

Der Zugang zur Praxis sowie zu den praxisbezogenen Zielen der TU Darmstadt erfolgt durch die Betrachtung der Studienordnung der Grundwissenschaften für das Lehramtsstudium an Gymnasien (TU Darmstadt 2006, 2009). Die Grundwissenschaften an der TU Darmstadt entsprechen strukturell den «Bildungswissenschaften» (KMK 2014) bzw. den «erziehungswissenschaftlichen Studien» (Terhart 2000; Horstkemper 2004). Dieser Zugang zur konkreten Praxis eröffnet zunächst die Möglichkeit zu hinterfragen, inwiefern die Ziele der TU Darmstadt mit den Zielstellungen der dargestellten Leitbilder vereinbar sind. Zudem wird es möglich, die Ziele für konkrete Module und den durchzuführenden Veranstaltungen in den Modulen im Rahmen der Grundwissenschaften verorten zu können. Mögliche Zielkonflikte werden im Rahmen einer vergleichenden Betrachtung der verschiedenen Zielebenen markiert. Eine Klärung der Frage, inwiefern die jeweiligen Zielstellungen von den handelnden Personen überhaupt legitim erscheinen und von diesen akzeptiert werden, erfolgt in einer abschließenden Positionierung.

Der Umfang der Grundwissenschaften umfasst an der TU Darmstadt 60 Leistungspunkte (LP). Diese $60 \mathrm{LP}$ verteilen sich auf sieben Studienmodule, «davon 4 Module mit insgesamt $33 \mathrm{LP}$ im Pflichtbereich und 3 Module aus 8 Modulen mit insgesamt 27 LP im Wahlpflichtbereich» (TU Darmstadt 2009, 5). Den «leitenden Studienzielen» entsprechend, so die Formulierung der Studienordnung (TU Darmstadt 2009, 3), umfasst der Pflichtbereich der Grundwissenschaften die folgenden Module: «P1 Grundlagen pädagogischen Denkens und Handelns» (9 LP), «P2 Didaktik, Methodik 
und Medien» (6 LP), «P3 Grundlagen der Psychologie des Lehrens und Lernens» (8 LP), sowie «P4 Schulpraktische Studien 1» (10 LP). Ferner sind im Wahlpflichtbereich "professionsspezifische Vertiefungen» möglich. Exemplarische Wahlpflichtmodule ${ }^{129}$ im Umfang von jeweils 9 LP sind u. a. «Informationspädagogik», «Selbstreflexion, Diagnostik und Beratung» oder «Pädagogik der Naturwissenschaften/Bildung für eine nachhaltige Entwicklung».

Für die Betrachtung der Studienordnungen der Grundwissenschaften der TU Darmstadt dient das Berufsleitbild «Lehrkräfte als Expertinnen und Experten für das Lehren und Lernen» als Referenzrahmen (Kap. 5.2.1). Im Vergleich der entsprechenden Zielstellungen mit der Studienordnung der Grundwissenschaften zeigen sich auf begrifflicher Ebene vielfältige Anknüpfungspunkte und Übereinstimmungen. Die Studienziele der Grundwissenschaften werden im Rahmen der Studienordnung wie folgt präzisiert:

«Das Studium der Grundwissenschaften soll den Studierenden als künftigen Lehrerinnen und Lehrern ein wissenschaftlich fundiertes und theoretisch reflektiertes Verständnis für die pädagogische Dimension ihrer beruflichen Tätigkeit vermitteln. Es fundiert und flankiert das Studium der Unterrichtsfächer hinsichtlich der besonderen Anforderungen, die über die fachlich korrekte Vermittlung von Inhalten hinaus in den Bereichen Unterrichten, Erziehen, Beraten, Betreuen und Innovieren mit dem Lehrerberuf verbunden sind. Weitergehend erweist sich die Wissenschaftlichkeit des Lehramtsstudiums nicht zuletzt in der Fähigkeit, die je gegebenen Verhältnisse und Strukturen im Bildungswesen in ihrer sozialhistorischen Gebundenheit zu erkennen und zu ihnen in eine kritische, neue Frei- und Gestaltungsräume eröffnende Distanz zu treten» (TU Darmstadt 2009, 3).

Das Ziel ist es, ein «wissenschaftlich fundiertes und theoretisch reflektiertes Verständnis für die pädagogische Dimension ihrer beruflichen Tätigkeit» (TU Darmstadt 2009, 3) zu erarbeiten. Die Metaphorik der wissenschaftlichen Fundierung» bietet zum einen Anknüpfungsmöglichkeiten an die Zielstellung zur Entwicklung einer wissenschaftlichen Wissensbasis (Terhart 2002, 21). Zum anderen wird die geforderte Entwicklung einer Reflexionsfähigkeit explizit in der Zielformulierung adressiert. Ferner findet sich die Entwicklung einer Reflexionsfähigkeit implizit in der Formulierung, Verhältnisse und Strukturen erkennen und zu ihnen in eine kritische Distanz treten zu können. So bezeichnet Meyer $(2003,101)$ die Fähigkeit, «Theorie- und Praxiswissen aufeinander zu beziehen und dadurch eine reflexive Distanz zur eigenen Berufsarbeit herzustellen», als Reflexionskompetenz. Weitere Anknüpfungspunkte

129 Zur Vollständigkeit beschäftigen sich die fünf weiteren Wahlpflichtmodule mit den folgenden Themen: «Genderforschung», «Erwachsenen- und Weiterbildung», "Angewandte Lehr- und Lernpsychologie», «Politische Rahmenbedingungen pädagogischen Handelns und Denkens», "Schule und Bildung im gesellschaftlichen und kulturellen Kontext». 
finden sich zudem in den Kompetenzformulierungen der Studienordnungen, welche die Studienziele näher bestimmen:

- «Bildungstheorien und ihr Verhältnis zu Gesellschaftstheorien kennen und Erziehungs- und Bildungsstandards danach einschätzen,

- Ergebnisse der Jugend- und Bildungsforschung sowie der Entwicklungspsychologie kennen und ihren Einfluss auf pädagogisches Handeln reflektieren,

- Verfahren und Ziele von Schulentwicklung beschreiben sowie Verfahren der Evaluation und Qualitätssicherung darstellen und einschätzen,

- Schule, Schulsystem und Lehrerberuf in historischen und gesellschaftlichen Zusammenhängen darstellen und reflektieren,

- Lernstrategien und Lernmethoden für Unterricht und Erziehung analysieren, begründen und bewerten,

- Vermittlungs- und Interaktionsprozesse für pädagogisches Handeln in Unterricht und Schule unter verschiedenen Bedingungen analysieren, begründen und bewerten,

- den Einsatz neuer Medien pädagogisch begründen und argumentativ vertreten,

- Prozesse und Maßnahmen der Koedukation, interkultureller sowie integrativer Erziehung und Bildung beschreiben und einschätzen,

- Heterogenität mit diagnostischen Mitteln erfassen und reflektieren,

- Konfliktsituationen und Kommunikationsstörungen in Unterricht und Erziehung analysieren und Bewältigungsstrategien darstellen und bewerten» (TU Darmstadt 2009, 3 f.).

Die Formulierung dieser Kompetenzen ist auf formaler Ebene insofern interessant, da im Vergleich zu den «Standards für die Lehrerbildung: Bildungswissenschaften» (KMK 2014) ein deutlich anderes Verhältnis zwischen Wissensvermittlung und der Entwicklung einer Reflexionsfähigkeit sichtbar wird. Zur Präzisierung der Kompetenzen in den Standards der KMK (2014) wurden vorzugsweise wissensbezogene Operatoren verwendet, während Operatoren für stärker reflexive Ansätze überraschend selten verwendet wurden. Im Gegensatz dazu zeigt sich in den Kompetenzformulierungen der Grundwissenschaften (TU Darmstadt 2009) ein ausgeglichenes Bild: Wissensbezogene und deskriptive Operatoren, z. B. «kennen» (2) und «beschreiben» (2) und darstellen (3) wurden insgesamt 8-mal verwendet.

Stärker an Reflexion orientierte Operatoren, z. B. «reflektieren» (3x), «analysieren» (3x) und «bewerten» (3x), wurden insgesamt 15 -mal verwendet. Vor diesem Hintergrund lässt sich auf der Ebene der Studienordnung der Grundwissenschaften zunächst festhalten, dass die hervorgehobenen Zielperspektiven, die Entwicklung einer wissenschaftlichen Wissensbasis sowie die Entwicklung von Reflexionsfähigkeit, vereinbar mit den Zielen des Studiums der Grundwissenschaften an der TU Darmstadt sind. Ferner erscheint die Bedeutung der Reflexionsfähigkeit in der Studienordnung der TU Darmstadt stärker gewichtet. 


\subsubsection{Erfahrungsbasierte Vorstellungen als Problem und Herausforderung}

Um mehr über die Erwartungen bzw. Einstellungen von Studierenden gegenüber dem Lehramtsstudium zu erfahren ${ }^{130}$, wird auf empirische Befunde zu den Lernvoraussetzungen von Lehramtsstudierenden zurückgegriffen (Blömeke 2004). Dieser zunächst abstrakte Zugang zur Perspektive der Studierenden eröffnet eine erste Orientierung zur Beantwortung der Frage, wie realistisch die zuvor markierten Zielstellungen für die Gruppe von Lehramtsstudierenden sind. In welcher Weise die Perspektive der beteiligten Studierenden in den Seminaren berücksichtigt wird, wie es in allgemeindidaktischen Planungsmodellen (z. B. Schulz 2006; Winkel 1997; Mayrberger 2014) gefordert wird, wird explizit beim Entwurf der Seminare diskutiert (Kap. 6.5.3). Das Ziel der folgenden Ausführungen ist es, mit Verweis auf empirische Befunde aufzuzeigen, welche Probleme und Herausforderungen sich für die konkrete Praxis der Lehrerinnen- und Lehrerbildung in Form von Lehrveranstaltungen ergeben.

Der Begriff der Lernvoraussetzungen umfasst für (Blömeke 2004) u. a. Einstellungen, Vorstellungen und Studienwahlmotive von Studierenden. Mit dem Fokus auf «Vorannahmen, Einstellungen bzw. Erwartungen» von Lehramtsstudierenden, die Blömeke $(2004,64)$ unter dem Begriff «Beliefs» zusammenfasst, lassen sich aus ihrer Perspektive die Ergebnisse empirischer Forschung wie folgt zusammenfassen:

- "Lehramtsstudierende treten mit Vorstellungen über schul- und unterrichtsrelevante Aspekte in die Lehrerausbildung ein.

- Diese beliefs sind erfahrungsbasiert. Ihr Zustandekommen lässt sich sozialisatorisch [...], evolutionär oder psychoanalytisch einordnen.

- Beliefs sind weitgehend veränderungsresistent, so dass es im Laufe der Lehrerausbildung eher selten zu grundlegenden Veränderungen kommt.

- Ihre Funktionsweise in der Ausbildung kann als Filter beschrieben werden. Es werden überwiegend nur solche Informationen aufgenommen, die sich in das vorhandene System an Überzeugungen einpassen lassen» (Blömeke 2004, 64 f.).

Die Erkenntnis, dass Lehramtsstudierende im Rahmen ihrer eigenen Schulzeit vielfältige Erfahrungen als Schülerinnen und Schüler sammeln, überrascht zunächst wenig. Auf Basis dieser Erfahrungen haben die Studierenden - in Abhängigkeit ihrer jeweiligen Schulen, Lehrerinnen und Lehrern und Mitschülerinnen und -schüler - unterschiedliche Vorstellungen davon entwickelt, was Schule und Unterricht ist bzw. wie Schule und Unterricht sein sollte. Die Herausforderung zum angemessenen Umgang mit erfahrungsbasierten Vorstellungen bzw. Überzeugen von Lehramtsstudierenden erläutert Pajares $(1992,323)$ im Vergleich mit anderen Studiengängen. Dafür greift er auf eine gegenüberstellende Metaphorik von «strangers» und «insiders»

130 Die eigene Lehre auch aus der Perspektive der Lernenden zu durchdenken, markiert eine Forderung des hochschuldidaktischen Leitbildes des Wandels vom Lehren zum Lernen (Schneider et al. 2009). Damit ist eine weitere normative Orientierung benannt, welche im Kapitel 4 thematisiert wird. 
zurück. Mit dem Begriff der «strangers» (englisch: Fremde) sind Studierende gemeint, die im Rahmen ihrer jeweiligen akademischen Disziplin kaum auf elaborierte Erfahrungen zurückgreifen können. Beispiele dafür sind Medizinstudierende in einer Notaufnahme oder einem Operationssaal sowie Jurastudierende in einem Gerichtssaal oder einer Anwaltskanzlei (Pajares 1992, 323). Sowohl die spezifischen Orte als auch die dort ablaufenden Prozesse und Handlungsweisen sind diesen Studierenden höchstwahrscheinlich fremd. Pajares $(1992,323)$ geht daher davon aus, dass diese Studierenden wahrscheinlich kaum über elaborierte Subjektiven Theorien verfügen bzw. kaum auf bewährte Überzeugungen zurückgreifen können. Dieses spezifische Verhältnis ist in der Argumentation von Pajares $(1992,323)$ eine hilfreiche Ausgangssituation zur Entwicklung eigener Vorstellungen:

«Accommodating new information and developing new beliefs are thus gradual enterprises of taking initial steps, accepting and rejecting certain ideas, modifying existing belief systems, and finally adopting new beliefs. For these strangers, the process involves minimal conflict or threat, for they have slight allegiance to prior expectations or ties to former practices and habits» (Pajares 1992, 323).

Im Gegensatz dazu lassen sich Lehramtsstudierende nach Pajares $(1992,323)$ als «insider» bezeichnen. Lehramtsstudierende sind aufgrund ihrer eigenen Schulzeit sehr vertraut mit Klassenzimmern und Schulen. Auch Lehrveranstaltungen in Hochschulen bieten strukturell vielfältige Ähnlichkeiten zu den Erfahrungen der eigenen Schulzeit. Diese Vertrautheit mit den bekannten Situationen wertet Pajares (1992, 323) als Problem, da sich für Lehramtsstudierende nicht in gleicher Weise die Herausforderung stellt, einen neuen Blick auf das bereits gewohnte Umfeld zu entwickeln. Vielmehr erleben sie die Hochschullehre und Unterrichtspraxis in der Logik ihrer Subjektiven Theorien bzw. in Anknüpfung an ihre jeweiligen Vorstellungen und Überzeugungen. Diese Konzepte und Vorstellungen zu wechseln, beschreibt Pajares $(1992,323)$ als potentiell belastend und bedrohlich: «For insiders, changing conceptions is taxing and potentially threatening. These students have commitments to prior beliefs, and efforts to accommodate new information and adjust existing beliefs can be nearly impossible».

Mit Verweis auf verschiedene Forschungssynthesen hebt Blömeke $(2004,65)$ auf inhaltlicher Ebene hervor, dass Lehramtsstudierende «sehr optimistisch sind, was ihre zukünftigen Fähigkeiten zu unterrichten angeht». Die beschriebene Filterfunktion der Beliefs der Lehramtsstudierenden konkretisiert Blömeke $(2004,65)$ in diesem Kontext wie folgt:

«Sie meinen zu wissen, wie Unterricht auszusehen hat (eine Lehrperson überliefert Wissen frontal vor der Klasse an die Schülerinnen und Schüler, die dieses rezeptiv aufnehmen), und sie meinen, nur noch ein bestimmtes 
Methodenrepertoire erlernen müssen, um starten zu können. Als besonders wichtig sehen es die Studierenden an, ihre Klassen im ‘Griff〉 zu haben. Gleichzeitig - und in einem gewissen Spannungsfeld hierzu - ist ihr Fokus auf soziale Faktoren des Unterrichtens ausgerichtet, indem sie ein verständnisvolles und sorgendes Verhältnis zu ihren Schülerinnen und Schüler für bedeutsam halten» (Blömeke 2004, 65).

Für Blömeke $(2004,65)$ wird damit die Annahme der Studierenden erkennbar, dass «die Persönlichkeit einer Lehrkraft von höherer Bedeutung sei als ihre kognitiven Fähigkeiten oder ihr pädagogisches bzw. fachliches Wissen». Im Vergleich der von Blömeke $(2004,65)$ pointiert formulierten Zusammenfassung der empirischen Befunde zu den Lernvoraussetzungen von Lehramtsstudierenden mit den zuvor formulierten Zielstellungen des Lehramtsstudiums kann ein deutliches Spannungsfeld markiert werden. So hat Horstkemper (2004) beispielsweise explizit hervorgehoben (und die Diskussion der Zieldimensionen bestätigt dies), dass die Entwicklung von Handlungsfähigkeit kein Ziel universitärer Lehrerinnen- und Lehrerbildung darstellt. Im Gegensatz dazu sind Studierende beispielsweise an der Vermittlung eines Methodenrepertoires interessiert, «um starten zu können» wie es Blömeke $(2004,65)$ formuliert. Im Fokus der Studierenden scheint vor allem berufsbezogenes Handlungswissen zu stehen. Im Gegensatz dazu wird eine wissenschaftliche Fundierung oder die Fähigkeit zur Reflexion des eigenen Denkens und Handelns von Blömeke (2004, 65) nicht explizit als Erwartung im Kontext der Lernvoraussetzungen benannt. Vor diesem Hintergrund erscheint es zweifelhaft, inwiefern das nach Horstkemper (2004) zu vermittelnde «Orientierungs- und Reflexionswissen» bzw. die Vermittlung eines "wissenschaftlich fundierte[n] und theoretisch reflektierte[n] Verständnis[ses] für die pädagogische Dimension ihrer beruflichen Tätigkeit» (TU Darmstadt 2009) aus studentischer Perspektive als relevant bzw. sinnvoll bewertet wird.

Die Bedeutung von Beliefs bzw. den erfahrungsbasierten Vorstellungen von Studierenden für das Lehramtsstudium wird ferner unter verschiedenen Perspektiven und Aspekten diskutiert. Horstkemper (2004) markiert die Herausforderung, Studierende für innovative Unterrichtsformen zu sensibilisieren und zu qualifizieren, wenn diese als Schülerinnen und Schüler selten entsprechende Erfahrungen gemacht haben. So werden nach Horstkemper (2004) «nur zu leicht [...] die eigenen Lernerfahrungen verlängert und unbefragt als orientierender Standard übernommen» (Horstkemper 2004, 470). Wildt (2003) diskutiert entsprechende Phänomene unter dem Begriff des pädagogischen Habitus. Für Wildt (2003) sind dabei nicht nur die Erfahrungen als Schülerinnen und Schüler von Bedeutung, sondern auch die erlebte Praxis der Hochschullehre. 
"Wie in der pädagogischen Praxis außerhalb, so auch in der Praxis der Lehre und des Studiums bildet sich [...] der pädagogische Habitus als eine Grammatik des Denkens, Fühlens und Handelns im pädagogischen Feld, der praktisches Handeln hervorbringt und sich selbst reproduziert «wie der Zug, der sich im Fahren seine Schienen selbst auslegt). Aufs Gleis gesetzt wird der Zug in den Kreisverkehr schulischer Lernmilieus gelenkt, durchläuft hochschulische Fachkulturen um wiederum in die routinemäßige Reproduktion der pädagogischen Praxis in Schule einzumünden. Die Prägung der schulischen Unterrichtswirklichkeit durch Frontalunterricht und Fragen-entwickelndes Unterrichtsgespräch erscheint stabil nicht zuletzt wegen ihrer Reproduktion durch die Form des pädagogischen Habitus an Hochschulen» (Wildt 2003, 77).

Mit dem Ziel, Probleme in der Praxis einer universitären Lehrerinnen- und Lehrerbildung im Kontext der Grundwissenschaften der TU Darmstadt zu identifizieren, kann eine erste Bilanzierung vorgenommen werden. Als zentrale Problematik erscheint die Diskrepanz zwischen den erfahrungsbasierten Vorstellungen der Studierenden sowie die damit verbundenen Erwartungen an das Studium der Grundwissenschaften einerseits und die in den Studienordnungen formulierten Ziele andererseits ${ }^{131}$. Wenngleich die Differenz zwischen fremdbestimmten Lehrzielen und selbstgesetzten Lernzielen ein bekanntes Spannungsfeld in verschiedenen formalen Bildungskontexten darstellt, eröffnet die von Blömeke $(2004,65)$ formulierte «Filterfunktion» erfahrungsbasierter Vorstellungen eine konkrete Bestimmung dieses Spannungsfeldes für die konkrete Praxis der erziehungswissenschaftlichen Studien in der Lehrerinnen- und Lehrerbildung. So stellen sich für die Gestaltung entsprechender Hochschullehre $u$. a. die Fragen, unter welchen Bedingungen und mit welchen Begründungen die Auseinandersetzung mit pädagogischem Theoriewissen aus studentischer Perspektive überhaupt als produktiv und sinnvoll erlebt werden kann.

Als eine zweite Problematik wurde mit Bezug auf Wildt $(2003,77)$ beschrieben, dass auch die Erfahrungen in der Hochschullehre Einfluss auf die Vorstellungen der Studierenden nehmen und im Sinne von Horstkemper (2004) «als orientierender Standard» dienen können. Am Beispiel einer Vorlesung im Bereich pädagogische Psychologie oder Allgemeine Didaktik, in der lerntheoretische Erkenntnisse über konstruktivistische Ansätze in einer fachsystematischen Struktur rezeptionsorientiert präsentiert werden, wird diese Problematik sichtbar. Wenngleich eine entsprechende Veranstaltung im Sinne der KMK (2014) wissenschaftlich fundiertes Fachwissen systematisch präsentiert, ist die Annahme naheliegend, dass Studierende im Sinne von (Wildt 2003, 77) eine Vorlesung als Bestätigung für «Frontalunterricht und Fragenentwickelndes Unterrichtsgespräch» erleben. Zugleich ist es fragwürdig, dass die

131 Diese Problematik ist gleichwohl mit der Annahme verbunden, dass die Studierenden an der TU Darmstadt den zusammengefassten Forschungsergebnissen und skizzierten Beobachtungen entsprechen. 
Studierenden die wissenschaftlichen Grundlagen zu konstruktivistischen Ansätzen in diesem Rahmen als relevantes Theoriewissen zur Entwicklung ihrer Reflexionskompetenz erkennen können.

\subsubsection{Zwischen normativen Zielen und erfahrungsfundierten Vorstellungen?}

Bei Betrachtung der Praxis der Lehrerinnen- und Lehrerbildung an der TU Darmstadt sowie unter Berücksichtigung der empirischen Befunde über Lernvoraussetzungen von Lehramtsstudierenden lässt sich vor dem Hintergrund der skizzierten Leitbilder eine zweite Problemdimension konkretisieren: Die leitbildkonformen Ziele der Grundwissenschaften der TU Darmstadt stehen in einem Spannungsverhältnis zu den erfahrungsbasierten Vorstellungen und Erwartungen von Lehramtsstudierenden. Mit dem Studienangebot der Grundwissenschaften wird das Ziel verfolgt, "ein wissenschaftlich fundiertes und theoretisch reflektiertes Verständnis für die pädagogische Dimension ihrer beruflichen Tätigkeit [zu] vermitteln» (TU Darmstadt 2009, 3). Studierende im Lehramtsstudium sind gemäss den empirischen Befunden jedoch vielmehr daran interessiert, in ihrer zukünftigen Praxis als Lehrerinnen und Lehrer handeln zu können und erhoffen sich dafür u. a. ein konkretes Methodenrepertoire. Für die Gestaltung konkreter Lehrveranstaltungen im Rahmen der Grundwissenschaften stellen sich $u$. a. die Fragen, unter welchen Bedingungen und mit welchen Begründungen die Auseinandersetzung mit pädagogischem Theoriewissen aus studentischer Perspektive überhaupt als produktiv und sinnvoll erlebt werden kann. Im Folgenden wird argumentiert, dass der Begriff der Reflexion bei verschiedenen Ansätzen zum Umgang mit der skizzierten Problemdimension eine zentrale Bedeutung hat. Zur Konkretisierung was Reflexion bzw. reflexives Lernen in der Lehrerinnenund Lehrerbildung bedeutet, wird ein Modellvorschlag von Wildt (2003) diskutiert.

Zum produktiven Umgang mit den erfahrungsbasierten Vorstellungen der Studierenden werden vielfältige Ansätze diskutiert (z. B. Blömeke 2004; Horstkemper 2004; Wildt 2003; Marsch 2009). Auf abstrakter Ebene markiert Blömeke $(2004,65)$ hinsichtlich der empirischen Befunde zu den Lernvoraussetzungen von Studierenden, dass Lehrprozesse erfolgsversprechend erscheinen «die aktiv an die vorhandenen beliefs anknüpfen und diese so schrittweise verändern». Horstkemper $(2004,470)$ verweist auf den Ansatz einer "biographisch orientierten Lehrerbildung», in der die eigenen Lehr- und Lernerfahrungen aufgearbeitet werden. Das Ziel dieses Ansatzes «ist die Schulung von Selbstreflexivität.» Wildt (2003) macht darauf aufmerksam, dass auch im Ansatz des forschenden Lernens (Obolenski 2003) die Reflexion der eigenen Rolle sowie der eigenen Erfahrungen auf verschiedenen Ebenen von Bedeutung sind. Gropengießer (2004) und Marsch (2009) diskutieren Metaphern des Lehrens und Lernens hinsichtlich der Möglichkeit, die eigenen Vorstellungen artikulieren zu können und so der eigenen Reflexion zugänglich zu machen. Der Begriff «Reflexion» wird in 
den verschiedenen Ansätzen in unterschiedlicher Weise thematisiert. Zur näheren Bestimmung und analytischen Verortung von Reflexion bzw. reflexivem Lernen in der Lehrerinnen- und Lehrerbildung wird das Modell von Wildt (2003) herangezogen (Abb. 5.2). Bei erster Betrachtung des Modells unterscheidet Wildt (2003) zunächst zwischen Theorie und Praxis.

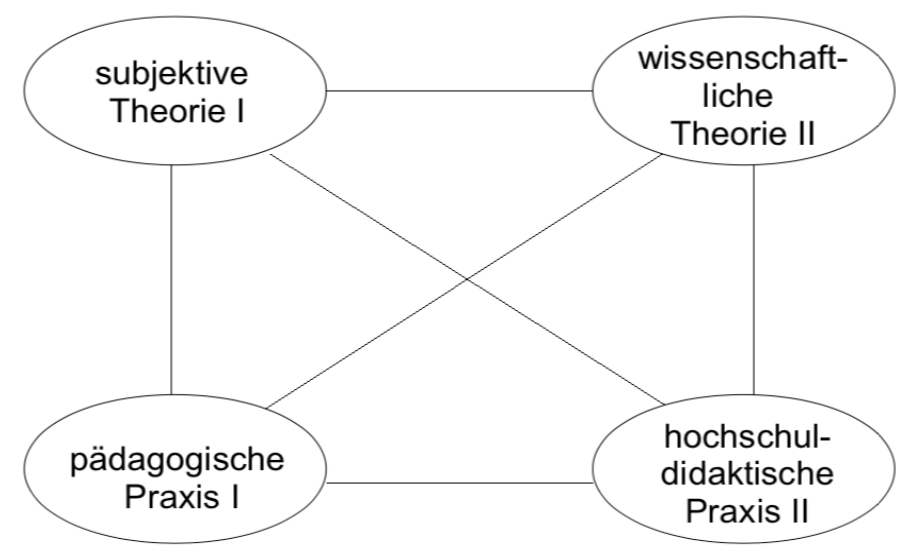

Abb. 5.2: Visualisierung der doppelten Theorie-Praxis-Relationierung nach Wildt $(2003,78)$.

In analytischer Betrachtung des Modells lassen sich diese zwei Facetten weiter differenzieren: Der Theoriebegriff beinhaltet nach Wildt (2003) einerseits die Subjektiven Theorien der Lehramtsstudierenden und andererseits die wissenschaftlichen Theorien der Erziehungswissenschaft. Dabei fasst der Begriff der Subjektiven Theorien jene Form von entwickelten Theorien und Vorstellungen, welche sich «die Subjekte dieser Praxis über angemessenes Handeln in der Praxis und sich selbst als Teil dieser Praxis bilden» (Wildt 2003, 75). Dieses Verständnis von Theorie ist anschlussfähig an die Ausführungen von Blömeke (2004), welche die Beliefs bzw. erfahrungsbasierten Vorstellungen von Lehramtsstudierenden als relevante Lernvoraussetzungen markiert (Kap. 5.2.2.2). So beschreibt auch Wildt (2003), dass sich Lehramtsstudierende "aufgrund langjähriger Schülererfahrungen [...] ein Bild von der Praxis gemacht und eine Vielfalt von mehr oder weniger überzeugenden Vorbildern kennengelernt [haben]». Von dieser Theorieform zu unterscheiden sind erziehungswissenschaftliche Theorien. Auf einer anderen Ebene reflektieren entsprechende Theorien, so Wildt (2003, 75), «pädagogische Praxis einschließlich der in ihr entwickelten [Subjektiven] Theorien». Zentrale Unterschiede dieser Theoriebegriffe markiert Wildt (2003) zudem hinsichtlich der «Beobachterposition» sowie der «Geltungsansprüche und Bewertungskriterien».

Den Begriff der Praxis unterscheidet Wildt (2003) mit den zwei Formulieren «Pädagogische Praxis I» und «Hochschuldidaktische Praxis I|». Pädagogische Praxis wird von Wildt (2003) vor allem als das schulische bzw. unterrichtliche Handlungsfeld von 
Lehrerinnen und Lehrern sowie von Schülerinnen und Schülern beschrieben ${ }^{132}$. Im Fokus seiner Beschreibungen erscheinen insbesondere die Handlungsroutinen bzw. -strategien von Lehrerinnen und Lehrern sowie von Schülerinnen und Schülern, welche sich im Handlungsfeld derart bewähren müssen, dass «diese weiter routinemäßig ausgeübt werden [können]» (Wildt 2003, 74). In Diskussionen der Lehrerinnen- und Lehrerbildung weitgehend vernachlässigt erscheint Wildt (2003) die Betrachtung der hochschuldidaktischen Praxis. Dabei lassen sich nach Wildt $(2003,77)$ verschiedene Parallelen markieren:

«In ihrer Lehrpraxis sind die Lehrenden an ihren Hochschulen nicht anders als die Lehrerinnen und Lehrer, die Studierenden nicht anders als die Schülerinnen und Schüler Teil einer pädagogischen bzw. didaktischen Praxis, als ebenfalls ceteris paribus in routineförmige Handlungsroutinen verkettet» (Wildt 2003, 77).

Bei Betrachtung des normativen Gehalts des Modells besteht für Wildt $(2003,78)$ die Aufgabe einer «um die hochschuldidaktische Dimension erweiterte Didaktik der Lehrerbildung» darin, «alle diese Relationen in den Blick zu nehmen». Reflexion bzw. reflexives Lernen kann insofern als Relationierungsaufgabe dieser unterschiedlichen Facetten verstanden werden. Diese Überlegungen sind anschlussfähig an die bisher skizzierten normativen Leitvorstellungen zur Bedeutung von Theoriewissen und der Entwicklung einer Reflexionsfähigkeit (z. B. Horstkemper 2004; Terhart 2009; Meyer 2003). Auf Basis des Modells von Wildt $(2003,78)$ eröffnet sich zudem die Möglichkeit, verschiedene Reflexionsebenen zu unterscheiden.

Eine erste Ebene verortet Wildt $(2003,75)$ in Bezug auf die pädagogische Praxis und (Subjektive) Theorien erster Ordnung und bezeichnet diese als «Reflexion im pädagogischen oder Erziehungssystem». Im Fokus steht das Verhältnis von pädagogischer Praxis und den Subjektiven Theorien der beteiligten Menschen über diese Praxis. Diese Reflexionsebene zielt auf das Durchdenken der praktischen Erfahrungen der beteiligten Menschen zur Entwicklung angemessener Lösungen für die Planung und Durchführung der jeweiligen Praxis. Ausgangspunkte «zur Reflexion als «Nachdenken» über das, was in der Praxis geschieht», so Wildt $(2003,74)$ weiter, «treten dann auf, wenn die Praxisroutinen im Alltag versagen, Krisen entstehen und neue Lösungen gefunden werden müssen». Möglichkeiten zur Auseinandersetzung mit dieser Reflexionsebene eröffnen sich im Rahmen des Lehramtsstudiums für Wildt (2003, 81) im Bereich «schulpraxis-bezogener Lehrveranstaltungen» wie beispielsweise schulpraktische Studien oder Projekte mit Schulen. Dabei markiert er zum einen die Relevanz, dass Studierende lernen, «die Ereignisse der Praxis zu entschlüsseln und

132 Der Begriff der Praxis wird dabei in unterschiedlicher Weise metaphorisiert. Beispielsweise als Behälter («in der Praxis»), als Person («die Belange der Praxis») oder als spezifische Handlungsmuster (z. B. «Praxisroutinen»). Gemeinsam ist diesen unterschiedlichen Bedeutungsaspekten, dass diese sich jeweils auf das Handeln von Lehrerinnen und Lehrern sowie den Lernenden in Schule und Unterricht beziehen. 
sich selbst als Teil dieser Praxis zu erkennen» (Wildt 2003, 81). Als mögliche Unterstützungsformate zu diesen Reflexionsaufgaben verweist Wildt $(2003,81)$ auf Beratungsformate wie kollegiale Beratung oder Supervision, welche sich im Kontext «schulpraxisbezogener Lehrveranstaltungen» realisieren lassen.

Eine zweite Reflexionsebene markiert Wildt $(2003,75)$ in Bezug auf erziehungswissenschaftliche Theorien (Theorie II) und deren Blick auf die «Theorie-Praxis-Relationierung» zwischen Subjektiven Theorien und pädagogischer Praxis. Diese Ebene wird von Wildt $(2003,75)$ als "Reflexion auf das bzw. über das Erziehungssystem» bezeichnet und unterscheidet sich im Vergleich zur ersten Ebene hinsichtlich der Urteilskategorien, des Gegenstandsbereiches sowie des Theoriebegriffs. So zielt die zweite Reflexionsform nicht auf die unmittelbare Lösung praktischer Probleme, sondern eröffnet die Chance zu Distanzierung von der ersten Reflexionsebene. Die Notwendigkeit dieser Reflexionsebene begründet Wildt $(2003,75)$ vor dem Hintergrund, dass die erste Reflexionsebene zwar «die Beurteilung der Angemessenheit praktischen Handelns fundieren [kann], [...] jedoch nicht die Überprüfung von Geltungsbehauptungen über Handlungszusammenhänge in der Praxis nach Wahrheitskriterien [gestattet]». Die auf der ersten Reflexionsebene gewonnenen «Sicherheiten», so Wildt (2003, 82), «schützen nicht vor trügerischer Selbsttäuschung». Möglichkeiten zur Auseinandersetzung mit dieser Reflexionsebene eröffnen sich im Rahmen des Lehramtsstudiums für Wildt $(2003,81)$ im Kontext des «forschende[n] Lernens» wie beispielsweise in einem Praxissemester.

\subsubsection{Zwischenfazit}

Für den Entwurf eines Seminars in den Grundwissenschaften der TU Darmstadt und deren systematische Analyse und Reflexion sind verschiedene Überlegungen zu berücksichtigen. Die getroffenen Aussagen zu den Lernvoraussetzungen in Form von Beliefs (Kap. 5.2.2.2) sind anschlussfähig an das Modell Wildt (2003) zur Relationierung von Subjektiven und wissenschaftlichen Theorien. Die individuellen Erfahrungen der Studierenden sind sowohl für das Konstrukt der Beliefs sowie für Subjektive Theorien zentral. Die Berücksichtigung dieser Erfahrungen in konkreten Seminaren erscheint ein geeigneter Ansatz zu sein, zur Relationierung von wissenschaftlichen Theorien und Subjektiven Theorien beizutragen. Diese Überlegungen sind vereinbar mit den Anforderungen an das Lehramtsstudium, sowohl eine Wissensbasis aufzubauen und die Entwicklung einer Reflexionsfähigkeit zu fördern. (Kap. 5.2.1).

Mit diesen Überlegungen sind zugleich verschiedene Fragen und Herausforderungen verbunden, welche in den folgenden Kapiteln näher thematisiert und diskutiert werden. Dafür erfolgt zunächst eine Auseinandersetzung mit den Forschungskonstrukten der Beliefs und der Subjektiven Theorien. Ebenfalls berücksichtigt wird das Konstrukt der metaphorischen Konzepte bzw. Denkfiguren. Bei vergleichender 
Betrachtung der drei Ansätze werden konzeptionelle Gemeinsamkeiten herausgearbeitet (Kap. 5.3). Das Reflexionspotenzial von Metaphern bzw. die Möglichkeit, mit Metaphern zur Relationierung von pädagogischem Theoriewissen und den erfahrungsfundierten Vorstellungen der Studierenden beizutragen, ist für den zu entwickelnden Entwurf interessant. Auf dieser Basis wurde entschieden, sich in den zwei folgenden Kapiteln den Forschungsstand von Metaphern in der Lehrerinnen- und Lehrerbildung (Kap. 5.4) sowie in der Pädagogik zu erarbeiten (Kap. 5.5). Die so gewonnenen Erkenntnisse dienen als Orientierung zur Formulierung eines begründeten Entwurfs (Kap. 6).

\subsection{Theoretisch-begriffliche Verortung}

Das folgende Kapitel widmet sich einer näheren Betrachtung der - bereits in der Problematisierung ${ }^{133}$ (Kap. 5.2) thematisierten - theoretischen Konstrukte «Beliefs» (Kap. 5.3.1), "Subjektive Theorien» (Kap. 5.3.2) sowie «metaphorische Konzepte» (Kap. 5.3.3). Für die drei Konzepte erfolgten jeweils eine begriffliche Diskussion sowie die Darstellung der zentralen konzeptionellen Annahmen. Zudem werden Anschlussstellen zu weiteren Forschungskonstrukten sowie mögliche methodische Zugänge aufgezeigt sowie die orientierende Funktion der Ansätze für die Praxis der Lehrerinnen- und Lehrerbildung skizziert. Eine Positionierung zu diesen Konstrukten und deren Relevanz im Rahmen dieser Arbeit erfolgt zum Abschluss dieses Kapitels (Kap. 5.3.4).

\subsubsection{Beliefs als Sammelbegriff}

In Anlehnung an Blömeke $(2004,64)$ wurde der Begriff Beliefs bisher als Sammelbegriff für «Vorannahmen, Einstellungen bzw. Erwartungen» verwendet. Die Begriffsverwendung begründet Blömeke $(2004,64)$ mit dem Hinweis auf das Fehlen «konsistenter Begrifflichkeit in der Forschung». Während diese Begriffsverwendung zur Problembestimmung der Praxis zunächst ausreichend war, lohnt es sich für die Aufarbeitung der theoretischen Grundlagen und der theoretischen Verortung den Begriff der Beliefs auf konzeptioneller Ebene näher zu bestimmen. Dafür werden im Folgenden auf die Erkenntnisse der zwei Übersichtsarbeiten von Pajares (1992) sowie Kane et al. (2002) Bezug genommen. Die Publikationen wurden aufgrund ihrer Verbreitung im

133 Der Begriff Subjektive Theorien wurde z. B. nach Wildt $(2003,75)$ als jene Form von entwickelten Theorien und Vorstellungen verstanden, welche sich «die Subjekte dieser Praxis über angemessenes Handeln in der Praxis und sich selbst als Teil dieser Praxis bilden». Über den Begriff der Vorstellungen sowie des impliziten Verweises auf Praxiserfahrungen ist diese Begriffsbestimmung von Wildt $(2003,75)$ anschlussfähig an Erkenntnisse über Beliefs in der Lehrerinnen- und Lehrerbildung nach Blömeke (2004) (Kap. 5.2.3). Beliefs von Lehramtsstudierenden werden dabei als erfahrungsbasierte Vorstellungen von Lehramtsstudierenden beschrieben, welche nach Blömeke (2004) als relevante Lernvoraussetzungen zu betrachten sind. 
wissenschaftlichen Diskurs ausgewählt. So wird zur Klärung des Verständnisses von Beliefs in zahlreichen Publikationen auf diese zwei Artikel zurückgegriffen ${ }^{134}$.

\subsubsection{Begriffliche Diskussion}

Auf begrifflicher Ebene kann mit Pajares (1992) und Kane et al. (2002) zunächst darauf hingewiesen werden, dass auch mit dem Begriff «Beliefs» das von Blömeke (2004, 64) problematisierte Fehlen konsistent verwendeter Begrifflichkeiten in der deutschsprachigen Forschung nicht gelöst werden kann. Als begriffliche Klammer umfasst der Begriff Beliefs bereits im englischsprachigen Kontext eine Vielzahl unterschiedlicher Aspekte und wird als psychologisches Konstrukt, wie Pajares $(1992,309)$ deutlich macht, auch unter anderen Begriffen und Synonymen diskutiert:

«They [beliefs] travel in disguise and often under alias-attitudes, values, judgments, axioms, opinions, ideology, perceptions, conceptions, conceptual systems, preconceptions, dispositions, implicit theories, explicit theories, personal theories, internal mental processes, action strategies, rules of practice, practical principles, perspectives, repertories of understanding, and social strategy, to name but a few that can be found in the literature» (Pajares 1992, 309).

Pajares (1992) zeigt in der Diskussion verschiedener empirischer und theoretischer Studien auf, dass Beliefs selten klar definiert oder explizit als konzeptionelles Konstrukt verwendet werden. Die Untersuchung von Beliefs in vielfältigen Kontexten und Bereichen, so die Vermutung von Pajares (1992), führte zu einer Vielzahl unterschiedlicher Begriffsbedeutungen.

Mit Pajares (1992) kann die Unterscheidung zwischen Beliefs und Wissen als Gemeinsamkeit vieler Arbeiten markiert werden. Exemplarisch für diese Unterscheidung steht der Artikel von Nespor (1987) zur Rolle von Beliefs in der Praxis. Bei Nespor (1987) lassen sich Unterschiede zwischen Beliefs und Wissen auf verschiedenen Ebenen identifizieren: (1.) allgemeine Gültigkeit und logische Konsistenz, (2.) Offenheit für Überprüfung und Veränderungen sowie (3.) Einfluss bzw. Relevanz für Handeln. So ging Nespor (1987) davon aus, dass Beliefs im Gegensatz zu Wissen hinsichtlich ihrer Gültigkeit und Angemessenheit nicht auf allgemein geteilten Annahmen oder übereinstimmenden Ansichten basieren müssen. Individuelle Überzeugungen erfordern nicht einmal eine innere Konsistenz zwischen verschiedenen Beliefs. Für Pajares (1992, 311) impliziert diese «Nichtkonsensualität», dass «belief systems are by their very nature disputable, more inflexible, and less dynamic than knowledge systems».

134 Der Beitrag von Pajares (1992) wurde gemäss Google Scholar 9821-mal und gemäss Sage Journals 1869-mal zitiert (Stand 13.07.2018). Dazu gehören u. a. Autorinnen und Autoren wie Baumert und Kunter (2006) oder Blömeke (2004). Der Zusammenfassung von Kane et al. (2002) wurde gemäss Google Scholar 797-mal und gemäss Sage Journals 199-mal zitiert (Stand 13.07.2018). 
Pointiert formuliert Pajares $(1992,311)$ die Unterscheidungsdimension von Nespor (1987) hinsichtlich der Offenheit für Überprüfung und Veränderungen von Wissen und Beliefs:

«Knowledge systems are open to evaluation and critical examination; beliefs are not. Nespor added that belief systems are also unbounded in that their relevance to reality defies logic, whereas knowledge systems are better defined and receptive to reason» (Pajares 1992, 311).

Während angenommen wird, dass Wissen durch rationale und begründbare Argumentationen sowie nachvollziehbare Beweise erweiterbar bzw. veränderbar ist, werden Beliefs als weitgehend unveränderlich beschrieben. Wenn es zu Veränderungen von Beliefs kommt, so erfolgt dies für Nespor (1987) nicht notwendigerweise auf Basis von Argumenten, sondern vielmehr als «conversion oder gestalt shift» (ebd., 321). Eine dritte Unterscheidung sieht Nespor (1987) in der Relevanz von Wissen und Beliefs hinsichtlich des praktischen Handelns als Lehrkraft. So formuliert Pajares (1992), dass Nespor (1987) davon ausging, dass Überzeugungen weitaus einflussreicher sind als Wissen hinsichtlich der Frage, wie Individuen Aufgaben und Probleme organisieren und definieren. Entsprechend können Beliefs als stärkere Prädiktoren für Verhalten betrachtet werden.

Beim Vergleich verschiedener Studien weist Pajares $(1992,311)$ darauf hin, dass der Unterschied zwischen Beliefs und Wissen eher graduell als kategorial erscheint und abhängig von der jeweiligen Operationalisierung der Forscherinnen und Forscher ist. Als Empfehlung für zukünftige Untersuchungen weist er darauf hin, dass Forscherinnen und Forscher mit einer gut begründeten, allgemein verständlichen und konsistenten Entscheidung gut bedient wären. Je nach Forschungsfrage ist dabei zu klären, in welcher Weise Beliefs und Wissen verstanden werden sowie die Relation zum Handeln bzw. Verhalten von Lehrerinnen und Lehrern sowie den Lernenden gedacht wird. Auf begrifflich-konzeptioneller Ebene skizziert Pajares $(1992,311)$ darüber hinaus weitere Probleme ${ }^{135}$, u. a. die Differenz zwischen «teachers' beliefs» und «educational beliefs». So wird die Formulierung von «teachers" beliefs» mitunter als scheinbar selbstverständliches Label oder Etikett verwendet, obwohl unter diesem Begriff konzeptionell sehr unterschiedliche Aspekte diskutiert werden.

Einstellungen, Überzeugungen sowie handlungsleitende Kognitionen von Lehrerinnen und Lehrern werden in der englischsprachigen Diskussion häufig unter dem Begriff «teachers" belief» zusammengefasst (Pajares 1992; Fussangel 2008). Dieser Begriff umfasst domänübergreifende Beliefs (z. B. bezogen auf Schule, Lehren und

135 Ein weiteres Problem kann in der Begriffsvielfalt gesehen werden. So geht Pajares $(1992,311)$ davon aus, dass «educational beliefs» von Lehrerinnen und Lehrern unter verschiedenen Begriffen, welche auf konzeptioneller Ebene vielfältige Gemeinsamkeiten aufweisen, diskutiert werden. So sind die Begriffe «teachers perspective», "preconceptions», «implicit theories» anschlussfähig an verschiedene Konzepte. 
Lernen) sowie domänspezifische Beliefs (z. B. bezogen auf einen spezifischen Fachunterricht). Aus der Perspektive von Pajares (1992) ist diese Begriffsverwendung aus mehreren Gründen problematisch. Das zentrale Argument ist, dass auch Lehrerinnen und Lehrer Beliefs zu Angelegenheiten jenseits ihres Berufs haben. Wenngleich diese ihre Praxis beeinflussen können, so Pajares (1992, 316), sollten sie nicht mit den schul- und bildungsspezifischen Überzeugungen verwechselt werden. Wenn in Publikationen der Begriff «teachers' belief» verwendet wird, bezieht sich dieser nur selten auf das allgemeine «belief system» von Lehrerinnen und Lehrern. Vielmehr stehen «educational beliefs» von Lehrerinnen und Lehrern im Fokus vieler Beiträge, welche zwar als Bestandteil eines allgemeinen «belief system» verstanden werden können, aber zugleich deutlich präziser auf die pädagogische Tätigkeit von Lehrerinnen und Lehrern gerichtet sind. Für Pajares $(1992,316)$ ist es zum einen wichtig, diese Unterscheidung zu treffen. Zum anderen weisen er darauf hin, dass auch das Konstrukt «educational beliefs» als ein sehr breites Konzept verstanden werden und für konkrete Forschungsarbeiten eingegrenzt werden muss. Exemplarisch unterscheidet Pajares $(1992,316)$ u. a.

«beliefs about confidence to affect students' performance (teacher efficacy), about the nature of knowledge (epistemological beliefs), about causes of teachers' or students' performance (attributions, locus of control, motivation, writing apprehension, math anxiety) [...]».

\subsubsection{Konzeptionelle Annahmen}

Auf konzeptioneller Ebene markiert Pajares (1992) 16 zentrale Annahmen, die bei der Untersuchung über Vorstellungen von Lehrerinnen begründbar anzunehmen sind (jedoch nicht als kategoriale Wahrheiten missverstanden werden sollten). Im Folgenden werden die Annahmen von Pajares (1992) übersetzt und thematisch gebündelt. Zur Entwicklung und zur Veränderung von Beliefs bzw. Überzeugungen trifft Pajares (1992) die folgenden Aussagen:

- Beliefs werden früh gebildet und tendieren dazu, sich selbst aufrechtzuerhalten und sogar gegen Widersprüche zu bestehen, die durch Vernunft, Zeit, Schulbildung oder Erfahrung verursacht werden (Pajares 1992, 324).

- Individuen entwickeln ein «belief system», das alle Überzeugungen aufnimmt, die durch den Prozess erworben werden, den Pajares $(1992,325)$ als «cultural transmission» bezeichnet.

- Manche Überzeugungen sind aufgrund ihrer Natur und Herkunft unwiderlegbarer als andere (ebd.).

- Je früher eine Überzeugung in die Struktur aufgenommen wird, desto schwieriger ist es, sie zu verändern. Neu erworbene Überzeugungen sind am anfälligsten für Veränderungen (ebd.). 
- Eine Überzeugungsveränderung im Erwachsenenalter ist ein relativ seltenes Phänomen. Menschen neigen dazu, Überzeugungsgrundsätze zu behalten, die auf falschem oder unvollständigem Wissen basieren, selbst wenn ihnen wissenschaftlich korrekte Erklärungen präsentiert werden (Pajares 1992, 326).

- Überzeugungen über das Unterrichten sind gut etabliert, wenn Studierende mit einem Lehramtsstudium beginnen (ebd.).

Zudem werden von Pajares (1992) eine Vielzahl von Annahmen getroffen, welche das Verhältnis von Beliefs zu Denk- und Wahrnehmungsprozessen beschreiben.

- Das Glaubenssystem hat eine adaptive Funktion, um den Menschen zu helfen, die Welt und sich selbst zu definieren und zu verstehen (Pajares 1992, 325).

- Wissen und Überzeugungen sind untrennbar miteinander verbunden. Zugleich wirken Überzeugungen aufgrund ihrer affektiven, evaluativen und episodischen Natur wie ein Filter, durch den neue Phänomene interpretiert werden (ebd.).

- Denkprozesse können durchaus Vorläufer und Schöpfer von Überzeugungen sein, aber die Filterwirkung von Überzeugungsstrukturen schliesslich schirmt, definiert, verzerrt oder formt die nachfolgende Denk- und Informationsverarbeitung neu (ebd.).

- Epistemologische Überzeugungen spielen eine Schlüsselrolle bei der Wissensinterpretation und dem kognitiven Monitoring (ebd.).

- Überzeugungen werden nach ihren Verbindungen oder Beziehungen zu anderen Überzeugungen oder anderen kognitiven und affektiven Strukturen priorisiert. Offensichtliche Inkonsistenzen können erklärt werden, indem die funktionalen Zusammenhänge und die zentrale Bedeutung der Überzeugungen untersucht werden (ebd.).

- Überzeugungen lassen sich als ein strukturiertes System mit verschiedenen Subkategorien verstehen. Pädagogische Überzeugungen lassen sich beispielsweise für Pajares $(1992,325)$ in Bezug zu ihren Verbindungen nicht nur untereinander, sondern auch mit anderen, vielleicht zentraleren Überzeugungen verstehen.

- Überzeugungen spielen eine entscheidende Rolle für die Organisation von Wissen und Informationen (Pajares 1992, 326).

- Überzeugungen beeinflussen stark die Wahrnehmung. Zugleich beschreibt Pajares sie als «unreliable guide to the nature of reality» (Pajares 1992, 326).

Die von Pajares (1992) zitierten Annahmen dienen im Folgenden dem Vergleich der konzeptionellen Gemeinsamkeiten und Unterschiede zu den Forschungskonstrukten der «Subjektiven Theorien» (Kap. 5.3.2) und metaphorischen Konzepte (Kap. 5.3.3). Eine Positionierung erfolgt zum Abschluss dieses Kapitels (Kap. 5.3.4). 


\subsubsection{Anschlussstellen zu Forschungskonstrukten und methodischen Zugängen}

Bei Betrachtung der begrifflichen Diskussion von Beliefs von Pajares (1992) sowie der formulierten definitorischen Merkmale lassen sich an dieser Stelle bereit erste Ähnlichkeiten zu weiteren Konstrukten benennen. Mit der Formulierung von «implicit theories», «explicit theories», "personal theories» subsummiert Pajares (1992) verschiedene Begriffe und damit verbundene theoretische Ansätze, welche beispielsweise von Groeben et al. (1988) unter dem Begriff Subjektive Theorien gefasst werden (Kap. 5.3.2). Die Begriffe «conceptions», «conceptual systems», «preconceptions» (Pajares 1992) lassen sich im deutschsprachigen Diskurs vor allem in der Fachdidaktik identifizieren. So geht es beispielsweise im Modell der didaktischen Rekonstruktion darum, die Vorstellungen von Lernenden zu identifizieren (Kap. 2.3.2). In Betrachtung der konzeptuellen Merkmale, beispielsweise hinsichtlich der Filterwirkung von Beliefs sowie ihrer orientierenden Funktion (Pajares 1992), lassen sich Ähnlichkeiten zum Konstrukt der metaphorischen Konzepte sowie Anschlussstellen zur kognitiven Metapherntheorie markieren. Die Auseinandersetzung mit Metaphern wird von Pajares $(1992,327)$ als eine von mehreren interessanten Forschungsperspektiven zur Untersuchung von Beliefs markiert: "Current interest in metaphor, biography, and narrative as ways to understand the beliefs of teacher and teacher candidates is a promising research direction» (Pajares 1992, 327).

Während sich auf begrifflicher und konzeptioneller Ebene Unterschiede zwischen den markierten Konstrukten von Beliefs, Subjektiven Theorien und Vorstellungen identifizieren lassen ${ }^{136}$, finden sich auch im deutschsprachigen Diskurs in ähnlicher Weise Beiträge, bei denen unter dem Begriff «Überzeugungen» weitere Konstrukte subsummiert werden. Dies zeigt sich beispielsweise in den Ausführungen von Baumert und Kunter (2006) zum Komplex «Überzeugungen/Werthaltungen» im Rahmen ihres Modells professioneller Handlungskompetenz von Lehrkräften ${ }^{137}$. Wenngleich Baumert und Kunter $(2006,497)$ den Anspruch formulieren, dass «systematische Wertbindungen (value commitments), epistemologische Überzeugungen (epistemological beliefs, world views), Subjektive Theorien über Lehren und Lernen sowie Zielsysteme unterschieden werden [sollen]», erfolgt diese Unterscheidung scheinbar nicht auf Basis der Begriffe Beliefs, Subjektive Theorien oder Wertbindungen. Vielmehr erscheinen diese Begriffe im Verlauf des Artikels synonym verwendet zu werden, wie an der Begriffsklärung epistemologischer Überzeugungen zeigt: «Unter den Begriffen «epistemologische Überzeugungen` (epistemological beliefs) oder «Weltbilder> (world views), so die Erklärung von Baumert und Kunter (2006, 498), «werden jene Vorstellungen und Subjektive Theorien subsumiert, die Personen über das Wissen und den Wissenserwerb generell oder in spezifischen Domänen entwickeln».

136 Dies wird im Folgenden zumindest für das Konstrukt der Subjektiven Theorien sichtbar.

137 Das Modell professioneller Handlungskompetenz von Baumert und Kunter $(2006,483)$ umfasst vier Komponenten (1.) Professionswissen (2.) Überzeugungen/Werthaltungen, (3.) Motivationale Orientierungen und (4.) Selbstregulative Fähigkeiten. 
Dass epistemologische Überzeugungen an dieser Stelle nur als Subsummierung von Vorstellungen und Subjektive Theorien verstanden werden, erscheint vor dem Hintergrund der systematischen Unterscheidung zumindest überraschend.

Für die empirische Untersuchung von Beliefs werden von Pajares (1992, 326) narrative und biographische Methoden sowie Forschungsansätze mit Metaphern als vielversprechend bezeichnet. Gleichwohl formuliert Pajares $(1992,328)$ das vielversprechende Potenzial entsprechender Ansätze vorsichtig. Für Forschungsansätze mit Metaphern begründet sich das Potenzial dieses Zugangs für Pajares $(1992,328)$ unter der Bedingung, dass sich die Annahme von Munby, «that metaphorical figures can offer insights into teachers ' construction of their professional reality», bestätigt. Wenngleich die Diskussion der Arbeiten von Munby auch kritisch diskutiert werden ${ }^{138}$, lassen sich vielfältige Forschungsprojekte identifizieren, in denen Metaphern explizit als forschungsmethodischer Zugang zu Überzeugungen von Lehrerinnen und Lehrern und Lehramtsstudierenden verwendet werden (z. B. Poom-Valickis et al. 2012; Kalra und Baveja 2012; Koc 2013; Alger 2009; de Guerrero und Villamil 2000 2002; Pinnegar et al. 2011; Leavy et al. 2007). Im Rahmen der Untersuchungen werden Metaphern einerseits als Instrument zur Datenerhebung und -auswertung verstanden. Anderseits wird in der Auseinandersetzung mit Metaphern ein reflexives Potenzial vermutet, welches die Versprachlichung (impliziter) Überzeugungen möglich macht.

\subsubsection{Orientierung für die Praxis}

Möglichkeiten und Herausforderungen zur Gestaltung der Lehrerinnen- und Lehrerbildung werden im Kontext von Forschungsarbeiten zu Überzeugungen von Lehrkräften und Lehramtsstudierenden auf makro- und mikrodidaktischer Ebene diskutiert. Auf makrodidaktischer Ebene eröffnen Erkenntnisse über die Überzeugungen von Lehramtsstudierenden für Pajares $(1992,328)$ wichtige Informationen zur Gestaltung zukünftiger Studiengänge und entsprechender Curricula. 16 Jahre nach der Veröffentlichung von Pajares Einschätzung bewerten Whitcomb et al. $(2008,268)$ die Entwicklung von neuen Lehrerinnen- und Lehrerbildungsprogrammen durchaus skeptisch. Pointiert formuliert erscheint Whitcomb et al. $(2008,268)$ insbesondere der Reflexionsbegriff in einigen Bildungsprogrammen zu einem Instrument geworden zu sein, um Studierenden programmatisch anerkannte Überzeugungen aufzuerlegen. Damit geht die Gefahr einher, dass zugunsten der Passung zu einer bestimmten Bildungsagenda die kritische Auseinandersetzung mit den persönlichen und sozialen Überzeugungen eingeschränkt wird. Entsprechend warnen Whitcomb et al. $(2008,268)$ davor, dass die Auseinandersetzung mit Überzeugungen nicht als

138 Beispielsweise problematisiert Schmitt $(2017,231)$, dass sich Munby in seinen Arbeiten «auf wenige, für wichtige gehaltene Metaphern» beschränkt wird. Forschungsmethodische Hinweise zur konkreten Durchführung der Metaphernanalysen finden sich kaum, so Schmitt (2017, 231). 
Indoktrinationsprozess missverstanden werden darf, sondern als Bildungsprozess verstanden werden muss. Damit eröffnet sich für Whitcomb et al. $(2008,268)$ ein Spannungsfeld, das in der Lehrerinnen- und Lehrerbildung berücksichtigt werden muss:

«Taking a few steps back, there appears to be a tension in our desire for an education that articulates and affirms beliefs on the one hand, and one that explores and challenges them on the other. We hold that a good teacher education program will encourage and address that tension - enabling teacher candidates to articulate and examine, as well as challenge and expand their educational beliefs. As one alternative, a transformative approach to teacher education appears to do that» (Whitcomb et al. 2008, 268).

Die in der Problematisierung (Kap. 5.2.3) benannten Ansätze zur Auseinandersetzung mit der eigenen Biografie (Horstkemper 2004, 470), zum forschenden Lernen (Wildt 2003) sowie zur Artikulation und Reflexion von Metaphern (Gropengießer 2004; Marsch 2009) eröffnen auf mikrodidaktischer Ebene Anknüpfungsmöglichkeiten zur Artikulation und Reflexion der eigenen Erfahrungen und Überzeugungen. Insbesondere die Entwicklung und Auseinandersetzung von Metaphern zur Untersuchung von pädagogischen Überzeugungen wird in zahlreichen englischsprachigen Publikationen auch als Möglichkeit zur Reflexion markiert. In Aufarbeitung des Forschungsstandes betonen de Guerrero und Villamil $(2000,341)$ den Konsens dahingehend, dass Metaphernanalysen eine ausgezeichnete Heuristik darstellen, um implizite Annahmen ins Bewusstsein zu rufen, zur Identifikation von Widersprüchlichkeiten ermuntern und letztendlich die Transformation von pädagogischen Überzeugungen und Praktiken fördern. Zugleich kann auf Basis der skizzierten Erkenntnisse darauf hingewiesen werden, dass sich zur Reflexion der eigenen Überzeugungen vielfältige Herausforderungen stellen. Dazu zählt beispielsweise die Frage, wie implizite Überzeugungen im Rahmen von Lehrveranstaltungen explizierbar oder zumindest rekonstruierbar werden können. Wenn Überzeugungen dazu tendieren, sich selbst aufrechtzuerhalten und sogar gegen Widersprüche zu bestehen, wie es Pajares (1992) skizziert, stellt sich die Frage, wie ein Hinterfragen der eigenen Überzeugungen möglich wird. In welcher Weise mit diesen Herausforderungen umgegangen werden kann, wird im Rahmen des theoriegeleiteten Entwurfs erneut aufgegriffen (Kap.6).

\subsubsection{Subjektive Theorien}

Der Begriff Subjektive Theorien wurden nach Wildt $(2003,75)$ bisher als jene Form von entwickelten Theorien und Vorstellungen verstanden, welche sich «die Subjekte dieser Praxis über angemessenes Handeln in der Praxis und sich selbst als Teil dieser Praxis bilden» (ebd.). Für die Aufarbeitung der theoretischen Grundlagen und 
der theoretischen Verortung ist es lohnenswert, Subjektive Theorien auf begrifflicher sowie konzeptioneller Ebene näher zu bestimmen. Das Begriffsverständnis von Groeben et al. (1988) eignet sich dafür als zentraler Ausgangspunkt, wie zahlreiche Dissertationen und Forschungsprojekte zu Subjektiven Theorien belegen (Wagner 2016; Hollick 2013; Wolf 2013; Fussangel 2008; Grzanna 2011; Blömeke et al. 2003; Stoffers 2015; Marsch 2009). Darüber hinaus werden Subjektive Theorien mit Verweis auf Dann (1989) diskutiert. Analog zur begrifflich-konzeptionellen Diskussion von Beliefs widmen sich die folgenden Ausführungen (1.) der Klärung grundlegender Annahmen zum Konstrukt Subjektiver Theorien, (2.) Möglichkeiten der methodischen Untersuchung, (3.) Anknüpfungspunkten zu weiteren theoretischen Konstrukten und (4.) didaktischen Ansätzen zum Umgang mit Subjektiven Theorien.

\subsubsection{Begriffliche Klärung und konzeptionelle Annahmen}

In ihrem Forschungsprogramm «Subjektive Theorien» explizieren Groeben et al. (1988) die Annahmen und Begriffsmerkmale ihres Forschungskonstruktes. Eine zentrale Prämisse dieses Konstruktes ist es, dass (Groeben et al. 1998, 11) - in Abgrenzung zu Forschung über Verhalten - das «Handeln von Menschen» als Ausgangspunkt ihrer Theoriebildung erklären. Damit verbunden ist ein Menschenbild, wie Wagner $(2016,11)$ zusammenfasst, «in dem - im Unterschied zum behavioristischen BlackBox-Modell - den Einschätzungen und Überlegungen des Menschen eine zentrale Rolle zukommt». Bei dieser Betrachtungsweise werden Menschen, so Wagner (2016, 11) weiter, vier Modellattribute zugeschrieben: «Reflexions-, Rationalitäts-, Kommunikations- und Handlungsfähigkeit». Wie Groeben et al. $(1988,12)$ methodologisch begründen, sind diese Vorannahmen für die Konzeptualisierung des Forschungsgegenstandes und dessen methodische Erforschung zentral und müssen entsprechend expliziert werden. Mit diesen Vorannahmen grenzen sich Groeben et al. $(1988,13)$ explizit von einem «mechanistischen Menschenbild» und deren Implikationen «der Umweltkontrolliertheit, des nicht-intentionalen, mechanistischen Funktionierens etc.» ab. Ein entsprechendes Menschenbild sehen Groeben et al. $(1988,13)$ im Behaviorismus sowie in «weiterführende[n] Theorieansätze[n] der künstlichen Intelligenz (‘Artificial Intelligence)) und Informationsverarbeitung (‘Cognitive Science)).» Groeben et al. $(1988,13)$ markieren das im Konstrukt der Subjektiven Theorien skizzierte Menschenbild bzw. Subjektmodell als Gegenentwurf.

Zur Explikation der begriffsbestimmenden Merkmale des Konstruktes Subjektive Theorie grenzen sich Groeben et al. (1988) kritisch vom Kognitionsbegriff ab. Groeben et al. $(1988,17)$ charakterisieren den Begriff der Kognition als zu umfassend und stellen dessen theoretische Präzision in Frage ${ }^{139}$. Zum produktiven Umgang mit

139 Wörtlich kritisieren Groeben et al. (1988) den Kognitionsbegriff als «zu aufgebläht»; «zu unpräzise» und "theoretisch als zu schwammig». 
diesem Problem argumentieren sie für eine «Binnenstrukturierung auf dem Gebiet der Kognitionspsychologie». Während unter dem Kognitionsbegriff in der Psychologie für Groeben et al. $(1988,18)$ meistens «einfache Phänomene» gefasst werden, kann das Konstrukt Subjektive Theorien für komplexe Zusammenhänge und Relationen einfacher Kognitionen verstanden werden, «denn damit sind auf jeden Fall komplexere Aggregate von Konzepten gemeint, deren Struktur und Funktion in Parallelität zu wissenschaftlichen Theorien konzipiert bzw. postuliert werden» (Groeben et al. 1988, 18). In der Diskussion weiterer Abgrenzungsmerkmale formulieren sie zusammenfassend vier begriffsbestimmende Merkmale von Subjektiven Theorien:

"[1.] Kognitionen der Selbst- und Weltsicht, [2.] als komplexes Aggregat mit (zumindest impliziter) Argumentationsstruktur, [3.] das auch die zu objektiven (wissenschaftlichen) Theorien parallelen Funktionen, [4.] der Erklärung, Prognose, Technologie erfüllt» (Groeben et al. 1988, 19).

In diesem weiten Begriffsverständnis von Subjektiven Theorien sehen Groeben et al. $(1988,19)$ ein hohes Integrations- und Rekonstruktionspotential vielfältiger theoretischer Ansätze, selbst wenn diese «ohne expliziten Rückgriff auf die Konzeption «Subjektive Theorie` ausgearbeitet wurden». Exemplarische historische Ansätze die unter dem Konstrukt Subjektive Theorien subsumiert werden können, sind für Groeben et al. (1988, 19 f.) unter anderem die «personal construct)-Theorie», «die 〈Implizite Persönlichkeitstheorie»», «Naive〉 oder Alltagstheorien» sowie «subjektive Berufstheorien». Für den Gegenstandsbereich von Lehr- und Lernprozessen werden empirische Studien und Ansätze markiert, die unter dem Begriffsverständnis Subjektiver Theorien subsummiert werden können. Zudem hat das «das Rekonstruktionspotential und die damit verbundene Integrationskraft» des Konstruktes Subjektive Theorien für Groeben et al. $(1988,21)$ dazu geführt, «dass (es) die meisten Vertreter eines FST das Konstrukt der 'Subjektiven Theorie) in dieser weiten Fassung vertreten».

In einem engen Begriffsverständnis erweitern Groeben et al. $(1988,22)$ die vorgestellte Definition um zwei weitere Aspekte: «das Merkmal der Feststellung der Rekonstruktionsadäquanz im Dialog-Konsens und das Merkmal der potentiellen Rationalität» (Groeben et al. 1998, 22). Diese zwei Merkmale lassen sich auf die Annahme zurückführen, dass die «Erkenntnis des Menschen (weil dieser ein «Objekt) ist, das - im Gegensatz zu «naturwissenschaftlichen` Gegenständen - über sich selbst reflektieren kann) immer auch (zumindest potentiell) Selbsterkenntnis ist» (Groeben et al. 1998, 21). Daraus ziehen Groeben et al. $(1988,22)$ zum einen die methodologischen Folgerungen, dass Menschen im Dialog mit anderen Menschen potenziell dazu fähig sind, die jeweils eigenen Selbst- und Weltsichten zur Sprache zu bringen 
bzw. artikulieren zu können ${ }^{140}$. Zum anderen gehen Groeben et al. $(1988,22)$ davon aus, dass mit den Forschungssubjekten geprüft werden kann, inwiefern die rekonstruierte Subjektive Theorie «als ‘objektive) Erkenntnis übernommen werden kann». Mit diesem engen Begriffsverständnis werden die Annahmen zum Menschenbild von Groeben et al. (1988) methodologisch deutlich ernsthafter berücksichtigt und die Möglichkeiten der methodischen Erfassung und Rekonstruktion Subjektiver Theorien gegenstandsbezogen deutlich präziser gefasst (und damit auch eingeschränkt). Diese Fokussierung führte zur Kritik, welche unter anderem von Blömeke et al. (2003) aufgenommen wurde, um für die Untersuchung von Subjektiven Theorien alternative Erhebungs- und Analyseverfahren zu diskutieren und zu erproben.

Während Groeben et al. (1988) Subjektive Theorien insbesondere für die psychologische Forschung diskutieren, werden für den Gegenstandsbereich der Lehrerinnen- und Lehrerbildung auch von Dann $(1989,247)$ verschiedene "Grundannahmen und Anliegen der Erforschung subjektiver Theorien» formuliert. Mit Verweis auf Groeben et al. (1988) formuliert Dann (1989, 248 f.) fünf Definitionsmerkmale Subjektiver Theorien, die nach Dann $(1989,248$ f.) weitgehend mit Merkmalen weiterer Autorinnen und Autoren übereinstimmen:

- "Subjektive Theorien stellen relativ stabile kognitive Strukturen (mentale Repräsentationen) dar, die gleichwohl durch Erfahrung veränderbar sind. [...]

- Subjektive Theorien sind teilweise implizit (z. B. nichtbewußtseinsfähige Selbstverständlichkeiten oder unreflektierte Überzeugungen), teilweise aber dem Bewußtsein des Handelnden zugänglich, so daß (sic) er darüber berichten kann. Dies ist jedenfalls unter spezifischen Bedingungen möglich [...]

- Subjektive Theorien besitzen ähnliche strukturelle Eigenschaften wie wissenschaftliche Theorien. Insbesondere enthalten sie eine zumindest implizite Argumentationsstruktur (z. B. Wenn-dann-Beziehungen), wodurch Schlußverfahren (sic) ermöglicht werden.

- Analog wissenschaftlichen Theorien erfüllen Subjektive Theorien die Funktionen (a) der Situationsdefinition i.S. einer Realitätskonstituierung; (b) der nachträglichen Erklärung (und oft der Rechtfertigung) eingetretener Ereignisse; (c) der Vorhersage (oder auch nur der Erwartung) künftiger Ereignisse; (d) der Generierung von Handlungsentwürfen oder Handlungsempfehlungen zur Herbeiführung erwünschter oder zur Vermeidung unerwünschter Ereignisse.

- Über die Funktionen analog wissenschaftliche[r] Theorien hinaus kommt Subjektiven Theorien eine handlungsleitende oder handlungssteuernde Funktion zu. Zumindest bestimmte subjektive Theoriestrukturen stellen einen bedeutenden Teil der Wissensbasis des Handelns dar, unter bestimmten

140 Vor dem Hintergrund, dass Groeben et al. $(1988,21)$ ihr FST für den Gegenstandsbereich der Psychologie konkretisieren, stehen für sie bei entsprechenden Reflexionen vor allem die «(alltags-)psychologischen Inhalte im Vordergrund [...] ohne daß (sic) dadurch übersehen wird, daß (sic) sich Subjektive Theorien natürlich im Prinzip auf alle Gegenstände erstrecken können». 
Bedingungen werden sie im Verlauf von Handlungen aktiviert. Zusammen mit anderen (z. B. emotionalen) Faktoren beeinflussen sie so das beobachtbare Verhalten im Rahmen zielgerichteten Handelns» (Dann 1989, 248 f.).

Die von Dann (1989) zitierten Annahmen dienen als Orientierung zum Vergleich des Konstruktes der Subjektiven Theorien mit den zwei weiteren Forschungskonstrukten der Beliefs (Kap. 5.3.1) und metaphorischen Konzepte (Kap. 5.3.3). Eine Positionierung erfolgt zum Abschluss dieses Kapitels (Kap. 5.3.4).

\subsubsection{Anschlussstellen zu Forschungskonstrukten und methodischen Zugängen}

In aktuellen Forschungsarbeiten und Publikationen wird auf diese definitorischen Merkmale bzw. grundlegenden Annahmen zum Konstrukt Subjektiver Theorien in unterschiedlicher Weise Bezug genommen. Während verschiedene Autorinnen und Autoren (z. B. Fussangel 2008; Wolf 2013; Wagner 2016; Müller 2007; Stoffers 2015; Münderlein 2013) sich zur Klärung, Adaption und Verortung des Konstruktes Subjektive Theorien auf Groeben et al. (1988) und bzw. oder Dann (1989) beziehen, beschränken sich anderen Autorinnen und Autoren (z. B. Hollick 2013) auf die Diskussion methodologischer und methodischer Implikationen des Forschungskonstruktes $^{141}$. Die Adaption und Verortung des Konstruktes Subjektive Theorien umfasst u. a. Entscheidungen ${ }^{142}$ für ein enges bzw. breites Begriffsverständnis, Abgrenzungen und Verknüpfungen zu weiteren Konstrukten wie Beliefs, Wissen und Vorstellungen (Fussangel 2008; Blömeke et al. 2003; Marsch 2009; Stoffers 2015; Münderlein 2013) sowie der Subsummierung Subjektiver Theorien unter abstrakteren Modellen und Begriffen wie beispielsweise handlungsleitende Kognitionen (Blömeke et al. 2003) oder als Facette professioneller Handlungskompetenz (Baumert und Kunter 2006).

In unterschiedlicher Weise werden von Autorinnen und Autoren Bezüge und Abgrenzungen zwischen Subjektiven Theorien und Beliefs formuliert. Fussangel $(2008,71)$ kommt beispielsweise beim Vergleich der definitorischen Merkmale der zwei Konstrukte Beliefs und Subjektive Theorien zu dem Schluss, im Rahmen ihrer Untersuchung die zwei Begriffe synonym zu verwenden. Subjektive Theorien bzw. Beliefs versteht Fussangel $(2008,71)$ als «Kognitionen der Selbst- und Weltsicht, die handlungsrelevant sind». Entsprechende synonyme Begriffsverwendungen finden sich auch in der englischsprachigen Debatte. So verwendet beispielsweise Kalra und Baveja (2012) die Begriffe «beliefs» und "personal theories» synonym. In weiteren Ausführungen werden Subjektive Theorien mithilfe des Überzeungsbegriffs

141 Vor dem Hintegrund, dass in den Arbeiten Subjektive Theorien jeweils als zentrale Untersuchungsgegenstände ausgewiesen werden, erscheint der Verzicht auf eine begriffliche Präzisierung überraschend.

142 Die Entscheidung für ein enges bzw. breites Begriffsverständnis wird zum einen explizit diskutiert und ist zum anderen über die formulierte Begriffsklärung sowie über die methodischen Zugänge erkennbar. 
erläutert, ohne explizit auf das Konstrukt Beliefs zu verweisen (z. B. Blömeke et al. 2003; Wagner 2016). Wagner $(2016,10)$ unterscheidet subjektive und wissenschaftliche Theorien dahingehend, dass «subjektive Theorien zunächst Überzeugungssysteme einer Person sind». Im Vergleich dazu, so Wagner $(2016,10)$, «handelt es sich bei wissenschaftlichen Theorien meist um Überzeugungssysteme einer Gruppe von Wissenschaftlern». Auch Blömeke et al. $(2003,108)$ beschreiben Subjektive Theorien als «komplexe Überzeugungen des Individuums zu grundlegenden Fragen des Lehrens und Lernens». Ein expliziter Bezug auf das Konstrukt der Beliefs findet sich in den zitierten Arbeiten nicht.

In weiteren (häufig fachdidaktischen) Arbeiten werden Subjektive Theorien in Beziehung zu Vorstellungen bzw. conceptions diskutiert. Fussangel (2008) grenzt Subjektive Theorien beispielsweise von Misconceptions bzw. Fehlkonzepten ab und verweist auf Ansätze zum «Conceptual Change» zur Veränderung von Subjektiven Theorien. Münderlein (2013) verwendet den Begriff der Vorstellungen zur Erläuterung Subjektiver Theorien bzw. subjektive Perspektiven ohne dabei auf Forschungskonstrukte zu verweisen. So entscheidet sich Münderlein $(2013,61)$ dazu, «die Begriffe ‘Alltagstheorie` und ‘Subjektive Theorie` sowie die Begriffe «Verständnis` und 〈Handlungslogik〉 nahezu synonym [zu verwenden]». Sie versteht die Begriffe als «Platzhalter» für die empirisch relevanten Perspektiven der Akteure (Vorstellungen, Motive, Haltungen etc.)» (Münderlein 2013, 62), die im Rahmen ihrer Arbeit inhaltlich ausdifferenziert werden. Marsch (2009) und Stoffers (2015) verwenden den Vorstellungsbegriff von Gropengießer (1997, 2006). Dieser eröffnet die Möglichkeit zur Verortung subjektiver Theorien hinsichtlich unterschiedlicher Komplexitätsebenen von Kognitionen bzw. kognitiven Strukturen. Diese Möglichkeit führt Stoffers (2015, 24) mit dem Vorschlag von Groeben et al. $(1988,17)$ zur Binnenstrukturierung des Kognitionsbegriffs entlang der Dimension von einfach zu komplex zusammen. Die Unterscheidung der Komplexitätsebenen von Vorstellungen nach Gropengießer (1997, 2006) beinhaltet dabei vier Ebenen: Begriff, Konzept, Denkfigur und Theorie. Subjektive Theorien können nach Stoffers $(2015,24)$ als «die höchste Komplexitätsform» in diesem Schema verstanden werden. In ähnlicher Weise unternimmt Marsch (2009) den Versuch, unterschiedliche Metaphernbegriffe in das System der Komplexitätsebenen von Vorstellungen nach Gropengießer $(1997,2006)$ einzuordnen, um eine Anschlussfähigkeit zum Vorstellungskonzept aufzuzeigen.

Für die empirische Erhebung und Rekonstruktion von Subjektiven Theorien orientieren sich verschiedene Autorinnen und Autoren einerseits am engen Begriffsverständnis und der damit verbundenen methodischen Implikationen (z. B. Wagner 2016; Hollick 2013; Grzanna 2011). Anderseits lassen sich vielfältige weitere Verfahren identifizieren, die als Rekonstruktion Subjektiver Theorien beschrieben werden. Müller (2007) führt zur Rekonstruktion von Subjektiven Theorien von Erzieherinnen und Erziehern zum Bildungsbegriff episodische Interviews durch und wertet diese 
in Form unterschiedlicher Kodierverfahren aus. Blömeke et al. (2003) diskutieren die Kombination von Videostudien zur Erfassung von Unterrichtsskripts sowie die Durchführung von Interviews zur Rekonstruktion Subjektive Theorien. Dabei wird insbesondere die Beziehung zwischen Subjektiven Theorien und Unterrichtsskripts untersucht. Marsch (2009) vergleicht die empirischen Möglichkeiten zur Verwendung von expliziten Metaphern im Rahmen von Interviews sowie Ansätze der systematischen Metaphernanalyse zur Analyse der Vorstellungswelt von Biologielehrkräfte. Die exemplarisch benannten Untersuchungsansätze zeigen zum einen die Vielfalt an Möglichkeiten auf. Zum anderen darf nicht vergessen werden, dass sich das Begriffsverständnis von Subjektiven Theorien in den Untersuchungen durchaus unterscheiden kann. Mit Bezug auf das enge Begriffsverständnis von Groeben et al. (1988) formuliert Wagner $(2016,13)$ : «Wenn wir von subjektiven Theorien von Lehrerinnen und Lehrern sprechen, dann sollten auch die untersuchten Personen deren (subjektive) Korrektheit bestimmen und nicht (monolog-hermeneutisch) außenstehende Beobachter». Während die Angemessenheit des Begriffsverständnisses durchaus kontrovers diskutiert wird, besteht Einigkeit darin, dass auf die Gegenstandsangemessenheit der angewandten Erhebungs- und Auswertungsmethoden zu achten ist.

\subsubsection{Orientierung für die Praxis der Lehrerinnen- und Lehrerbildung}

Ansätze zum Umgang mit Subjektiven Theorien werden in der Literatur auf verschiedenen Ebenen diskutiert. Zum einen wird darauf hingewiesen, dass Subjektive Theorien aufgrund ihrer skizzierten Funktionen und definitorischen Merkmale als stabile Kognitionen verstanden werden (z. B. Dann 1999; Fussangel 2008; Marsch 2009). Eine Veränderung entsprechend stabiler und für die jeweiligen Menschen bewährter Subjektiver Theorien kann nicht nur durch die Präsentation neuen Wissens erfolgen. Fussangel (2008, 86 f.) kommt in Betrachtung verschiedener Forschungsergebnisse zu dem Schluss,

«[...] dass eine Veränderung von Subjektiven Theorien bzw. Beliefs dann stattfindet, wenn eine Person mit neuen Wissenselementen konfrontiert wird, neue Erfahrungen sammelt oder ihre bisherigen Annahmen unter einer neuartigen Perspektive betrachtet, die mit den bisher bestehenden Elementen ihrer Subjektiven Theorien nicht vereinbar sind» (Fussangel 2008, 86 f.).

Dabei stellen sich Fragen und Herausforderungen, z. B. inwieweit Lehrkräfte und Lehramtsstudierende in der Lage sind, die eigenen Subjektiven Theorien überhaupt explizieren zu können bzw. in welchen Lehr- und Lernarrangements die Artikulation eigener Subjektiver Theorien unterstützt werden kann. Werden Subjektive Theorien als komplexe Form von Vorstellungen verstanden (z. B. Stoffers 2015; Marsch 2009), erscheint die Auseinandersetzung mit Metaphern diesbezüglich sinnvoll. So kommt 
Marsch $(2009,109)$ in der Zusammenfassung ihrer Dissertation zu dem Schluss: «Metaphern können als Werkzeuge zur Reflexion und Veränderungen von Vorstellungen über das Lehren und Lernen genutzt werden» (ebd.). Dieses Potenzial sieht Marsch $(2009,106)$ «vor allem im Bereich der Lehrerfort- und -ausbildung» und führt verschiedene Überlegungen zur Ausbildung von Biologielehrkräften auf Basis des aktuellen Diskurses und ihrer Forschungsergebnisse vertiefend aus.

\subsubsection{Metaphern, metaphorische Konzepte und Denkfiguren}

In den bisherigen Ausführungen zur Problematisierung und offenen Gestaltungsmöglichkeiten (Kap. 5.2.3) sowie zu den theoretischen Annahmen und Grundlagen von Beliefs (Kap. 5.3.1) und Subjektiven Theorien (Kap. 5.3.2) wurde jeweils auf den Begriff der Metaphern verwiesen. Metaphern wurden dabei ein reflexives Potenzial zugesprochen. Sie wurden zudem als spezifische Form der Datenerfassung und -analyse Subjektiver Theorien und Beliefs betrachtet. Offen geblieben ist bisher die Frage, was unter dem Begriff Metapher in den verschiedenen Ansätzen eigentlich verstanden wird. Für die Aufarbeitung der theoretischen Grundlagen und der theoretischen Verortung gilt es im Folgenden, den Begriff der Metapher sowie die damit einhergehenden Konstrukte der metaphorischen Konzepte und Denkfiguren auf begrifflicher sowie konzeptioneller Ebene näher zu bestimmen. Die bisher zitierten Arbeiten, in denen Metaphern zur Erhebung und Analyse von Beliefs, Subjektiven Theorien und Vorstellungen verwendet wurden, orientieren sich (mehr oder weniger streng) an der kognitive Metapherntheorie nach Lakoff und Johnson (1980). Die Ausführungen von Lakoff und Johnson $(1980,2014)$, welche auch in weiterführenden deutschsprachigen Arbeiten als zentrale Metapherntheorie ausgewiesen wird (z. B. Gansen 2010; Schmitt 2017) stellen im Folgenden einen zentralen Ausgangspunkt dar. Dafür werden insbesondere die deutschsprachigen Weiterentwicklungen und Adaptionen der kognitiven Metapherntheorie von Schmitt (2017) und Gropengießer (2004, 2007) aufgegriffen. Schmitt (2017) hat den linguistischen Ansatz von Lakoff und Johnson $(1980,2014)$ für die sozialwissenschaftliche Forschung aufgearbeitet. Gropengießer (2007) hat mit der Theorie des erfahrungsbasierten Verstehens das Konzept für fachdidaktische Forschungs- und Entwicklungsprojekte aufgearbeitet, indem er die Analyse von Metaphern als Zugang zur Vorstellungswelt von Lernenden erklärt. An die bisherigen Ausführungen zu Beliefs und Subjektiven Theorien anknüpfend, widmen sich die folgenden Ausführungen (1.) der Klärung grundlegender Annahmen zu den Begriffen Metaphern, metaphorisches Konzept und Denkfiguren, (2.) Möglichkeiten der methodischen Untersuchung, (3.) Anknüpfungspunkten zu weiteren theoretischen Konstrukten und (4.) didaktischen Ansätzen zur Gestaltung von Lehr- und Lernsituationen mit Metaphern. 


\subsubsection{Begriffliche Klärung und konzeptionelle Annahmen}

Lakoff und Johnson (1980) bieten eine, wie es Schmitt $(2017,39)$ beschreibt, «radikal einfache Definition» einer Metapher: «The essence of metaphor», so formulieren es Lakoff und Johnson $(1980,5)$, «is understanding and experiencing one kind of thing in terms of another». In der deutschsprachigen Übersetzung heisst es: «Das Wesen der Metapher besteht darin, dass wir durch sie eine Sache oder einen Vorgang in Begriffen einer anderen Sache oder einen Vorgang verstehen und erfahren können» (Lakoff und Johnson 2014, 13). Das Verstehen und Erfahren eines Phänomens in Begriffen eines anderen Phänomens steht für einen Metaphernbegriff, welcher weit über das Verständnis von Metaphern als rhetorisches Stilmittel hinausgeht. Metaphern sind in diesem Verständnis nicht nur ein sprachliches Mittel und beschränken sich nicht auf unser Sprechen bzw. unsere Sprache. In dieser Perspektive ist vielmehr «unsere Sprache und vor allem unser Denken metaphorisch strukturiert» (Gropengießer 2007, 105). Schmitt (2017) fasst zentrale Annahmen der kognitiven Metapherntheorie wie folgt zusammen:

«Lakoff und Johnson interessieren sich nicht für interessante), rhetorisch oder literarisch auffällige Metaphern, sondern die der Alltagssprache. Diese alltäglichen Metaphern sind für den von Lakoff und Johnson begründeten Denkansatz auch nicht als einzelne Redewendungen interessant, sondern als Dokumente gemeinsamer «kognitiver» Strukturen» (Schmitt 2017, 40).

Lakoff und Johnson (2014, 11 f.) gehen davon aus, dass unsere «Kommunikation auf dem gleichen Konzeptsystem beruht, nachdem wir denken und handeln». Sprache ist in diesem Sinne eine Ausdrucksform unseres Denkens. In diesem Verständnis eröffnet die Analyse unserer Sprache die Möglichkeit, Rückschlüsse auf unser Konzeptsystem zu ziehen. Durchaus metaphorisch beschreibt Gropengießer $(2007,105)$ dieses Verhältnis von Sprechen und Denken wie folgt: «Sprache ist ein Fenster auf unsere Kognition. Sprache enthüllt die Art und Weise, wie wir denken». Metaphern und metaphorische Konzepte sind dabei jedoch nicht nur auf den intellektuellen und wohl überlegten Sprachgebrauch beschränkt. Vielmehr gehen Lakoff und Johnson (2014, 11 f.) davon aus, dass auch unser «nichtreflektiertes Alltagshandeln» sowie unsere «Alltagssprache» weitgehend durch metaphorische Konzepte strukturiert sind. Zur Erläuterung dieses Begriffsverständnisses verwenden Lakoff und Johnson $(2014,12)$ das metaphorische Konzept «ARGUMENTIEREN IST KRIEG» und skizzieren verschiedene exemplarische sprachliche Ausdrücke:

«lhre Behauptungen sind unhaltbar.

Er griff jeden Schwachpunkt in meiner Argumentation an.

Seine Kritik traf ins Schwarze.

Ich schmetterte sein Argument ab.

Ich habe noch nie eine Auseinandersetzung mit ihm gewonnen. 
Sie sind anderer Meinung? Nun, schießen Sie los!

Wenn du nach dieser Strategie vorgehst, wird er dich vernichten.

Er machte alle meine Argumente nieder.» (Lakoff und Johnson 2014, 12; Hervorhebung im Original)

Das Beschreiben von Argumentationshandlungen als kämpferische bzw. kriegerische Handlungen ist für Lakoff und Johnson (2014, 12 f.) ein Beispiel für ein metaphorisches Konzept. Wenngleich es sich nicht um einen «physischen Kampf handelt», belegen die verschiedenen Formulierungen für Lakoff und Johnson (2014, 12 f.), dass Argumentieren als «ein verbaler Kampf» versprachlicht wird. Diese verschiedenen einzelnen metaphorischen Redewendungen und Begriffe, deren metaphorischer Gehalt vermutlich nicht auf den ersten Blick ersichtlich ist ${ }^{143}$, enthüllen darüber hinaus unsere Vorstellung von Argumentieren bzw. die Art und Weise wie wir Argumentieren verstehen und erfahren. Für Lakoff und Johnson $(2014,13)$ strukturiert das metaphorische Konzept auch «die Handlungen, die wir beim Argumentieren ausführen» bzw. stellt eine Orientierung dar, «nach der wir unserer Kultur leben» (ebd.). In Anlehnung an die Darstellungsform von Hager (2013) lässt sich das Verhältnis zwischen Denken und Sprache am zuvor skizzierten metaphorischen Konzept exemplarisch veranschaulichen (Abb. 5.3).

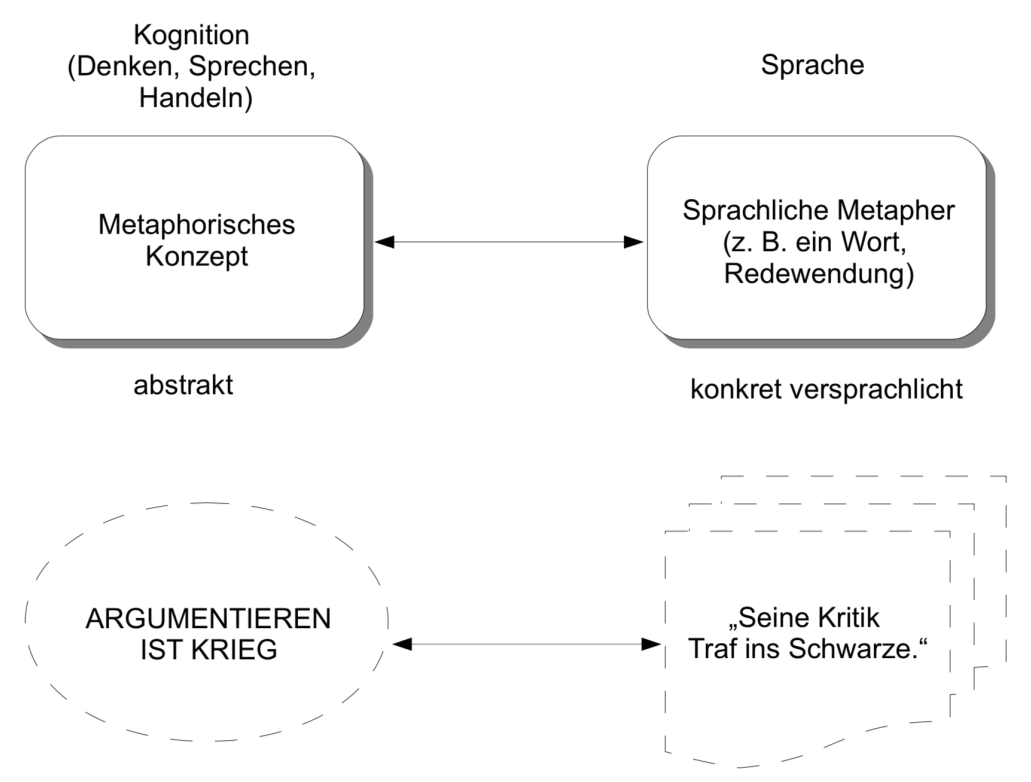

Abb. 5.3: Visualisierung des Verhältnisses von Denken und Sprache in der kognitiven Metapherntheorie in Anlehnung an Hager (2013).

143 Im Fokus ihrer Betrachtung steht vor allem die Alltagssprache. Zur Identifikation inwiefern ein Wort metaphorisch zu verstehen ist, lässt sich mit Schmitt (2017) jeweils fragen, inwiefern das zu prüfende Wort in der jeweiligen Situation mehr als nur eine wörtliche Bedeutung hat. 
Zur Differenzierung und Klärung der bisher verwendeten Begriffe lohnt es sich, zunächst zwischen einer konkret versprachlichten Metapher einerseits sowie einem metaphorischen Konzept andererseits zu unterscheiden. Während diese analytische Trennung bei Lakoff und Johnson (2014) und Gropengießer (2007) mitunter vage erscheint, bietet Schmitt (2017) eine konsistente Differenzierung. Eine Metapher wird entsprechend im Folgenden als Bedeutungsübertragung «von einem Bereich auf einen anderen» (Schmitt 2017, 39) verstanden. Eine konkret versprachlichte Metapher liegt dann vor, wenn ...

«ein Wort, eine Redewendung oder eine szenische Narration in einem strengen Sinn in dem für die Sprechäußerung relevanten Kontext mehr als nur wörtliche Bedeutung hat, die wörtliche Bedeutung einem für den Sprechenden prägnanten Bedeutungsbereich (Quellbereich) entstammt, jedoch auf einen zweiten, oft abstrakteren Bereich (Zielbereich) übertragen wird» (Schmitt 2017, 472).

Mit diesem weiten Verständnis und der relationalen Bestimmung von Metaphern wird erneut deutlich, dass «rhetorische Differenzierungen wie Sprichwort, Symbol, Chiffre, Vergleich und Allegorie» (Schmitt 2017, 472) aufgegeben werden. Vor dem Hintergrund der skizzierten Annahmen der kognitiven Metapherntheorie erscheint diese Unterscheidung weder für fachliche Lern- und Verstehensprozesse (Gropengießer 2007) noch für die qualitative Sozialforschung (Schmitt 2017) von besonderem Interesse. Mit dieser relationalen Bestimmung von Metaphern stellt sich für die Beurteilung von Wörtern, Redewendungen oder szenischen Narrationen die Frage, ob die jeweiligen Formulierungen wörtlich oder übertragen zu verstehen sind, wie Schmitt $(2017,39)$ exemplarisch demonstriert:

«So kann man wörtlich in einem «Glashaus` sitzen und sollte nicht mit Steinen um sich werfen, man kann metaphorisch in einem ¿Glashaus) sitzen (und sollte nicht mit Steinen des sozialen Anstoßes um sich werfen) und kann drittens wörtlich und metaphorisch zugleich im «Glashaus) sitzen. Alle drei Unterscheidungen sind davon abhängig, ob es einen Beobachter gibt, der mit seinem sprachlichen und situativen Verständnis diese Unterscheidung treffen kann» (Schmitt 2017, 39).

Die Möglichkeiten zur Bestimmbarkeit von Wörtlichkeit bzw. von Metaphern, wie Schmitt (2017) und Gansen (2010) zeigen, werden kontrovers diskutiert. Während sich verschiedene Probleme bei der Identifikation von Metaphern im Rahmen konkreter Analyseverfahren ergeben, wird die Gegenüberstellung von Wörtlichkeit und übertragener Bedeutung weder von Schmitt (2017) noch von Gansen (2010) aufgegeben. Mit Verweis auf die Arbeiten von Jäkel stellen beide Autoren fest, dass die Unterscheidung von Wörtlichkeit und Bedeutungsübertragung konstitutiv für Metaphorizität sind, wenngleich es keine trennscharfe Grenze zwischen diesen Phänomenen 
gibt. Die von Lakoff und Johnson (1980) formulierte «Unidirektionalitätshypothese» wie Schmitt $(2017,472)$ bezeichnet, bietet zur Unterscheidung allenfalls eine «leistungsfähige Regel», dessen Gültigkeitsbereich gleichwohl beschränkt ist. Dafür eröffnet Schmitt (2017, 472 ff.) eine Vielzahl an methodischen Orientierungshilfen zur Beurteilung von Wörtern, Redewendungen oder szenischen Narrationen.

Ein metaphorisches Konzept kann als zweiter zentraler Begriff der kognitiven Metapherntheorie beschrieben werden. Zur Begriffsbestimmung orientiert sich Schmitt $(2017,43)$ an der durch die kognitive Linguistik formulierte Annahme, dass «Metaphern in der Regel nicht ohne Zusammenhang auftreten, sondern sich bündeln lassen». Diese Bündelung kann aus verschiedenen metaphorischen Formulierungen, die Gemeinsamkeiten hinsichtlich des konkreten Quellbereichs sowie des abstrakten Zielbereichs aufweisen, rekonstruiert werden. Neben dem bisher skizzierten Beispiel "Argumentieren ist Krieg», kann mit der Abbildung 5.4 des metaphorischen Konzeptes «Erfolg ist ein Weg» bzw. "success is a path» von Moser $(2000,4)$ ein weiteres metaphorisches Konzept veranschaulicht werden. Auf Basis einer Interviewstudie zum Übergang vom Universitätsstudium ins Arbeitsleben visualisiert Moser (2000, 4) mithilfe von Interviewaussagen, die Übertragung von Begriffen eines konkreten Erfahrungsbereiches bzw. Quellbereichs (ein Weg gehen bzw. eines Start-Weg-ZielSchemas) auf einen abstrakten Zielbereich (Erfolg). Eine entsprechende Bündelung einzelner metaphorischer Redewendungen und Formulierung wird nach Schmitt $(2017,44)$ im Folgenden als «metaphorisches Konzept» verstanden ${ }^{144}$.

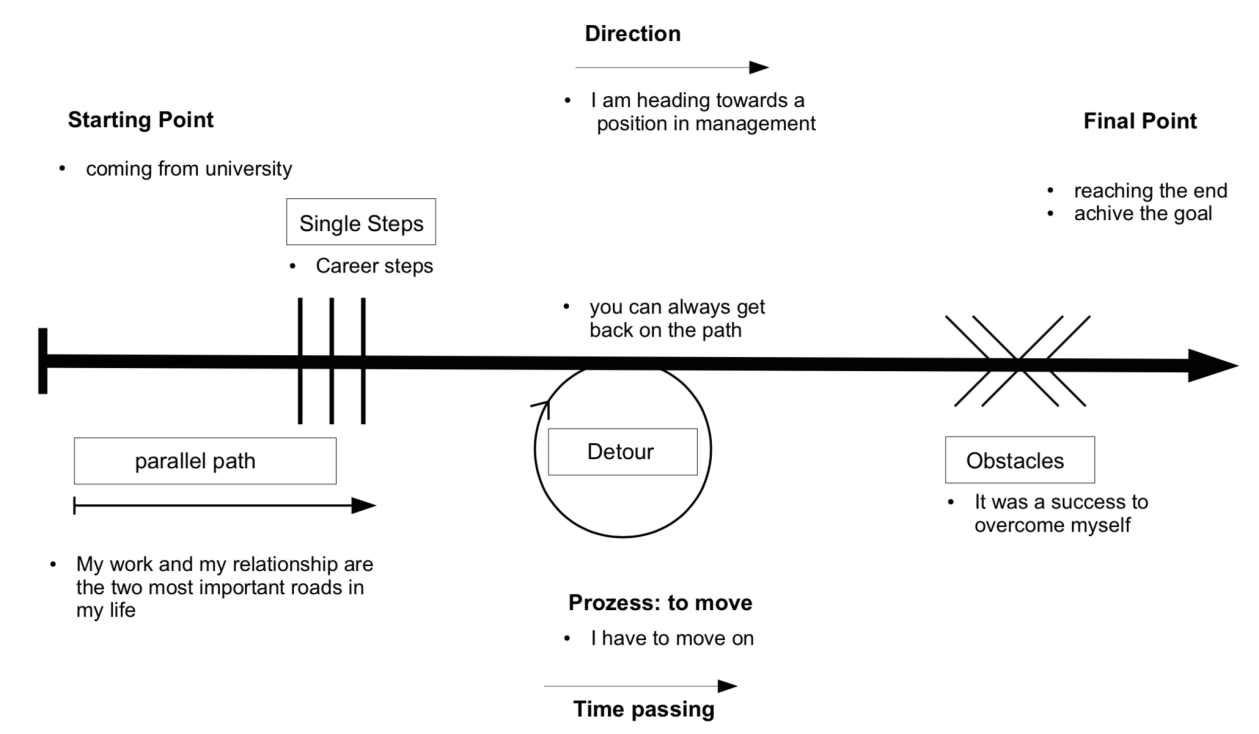

Abb. 5.4: Visualisierung des metaphorischen Konzeptes «success is a path» nach Moser (2000, 4).

144 Alternative Bezeichnungen sind u. a. «konzeptuelle Metapher» (Hager 2013), «conceptual metaphor» (Moser 2000), «Metaphernmodell» (Ryter Krebs 2008) oder «Denkfigur» (Gropengießer 2004). 
Die Rekonstruktion metaphorischer Konzepte erscheint u. a. Schmitt (2017, 45 f.) sinnvoll, um die «Überinterpretationen einzelner Metaphern zu vermeiden, weil ein breiterer semantischer Kontext als Beleg gesucht wird». Durchaus unterschiedlich wird die Gültigkeit bzw. werden die Geltungsgrenzen rekonstruierter metaphorischer Konzepte diskutiert. Das Spektrum reicht von kollektiven über spezifische (sub-) kulturelle bis zu individuellen metaphorischen Konzepten. In Abgrenzung von einer linguistischen Perspektive, wie sie von Lakoff und Johnson (2014) vertreten wird, geht es Schmitt $(2017,46)$ beispielsweise nicht darum, nach «gültigen Konzeptformulierungen für das gesamte Lexikon einer Sprache» zu suchen. Stattdessen verfolgt Schmitt $(2017,46)$ mit dem Entwurf seiner qualitativen Forschungsmethodik das Ziel, «möglichst gegenstandsangemessene, der Eigentümlichkeit der lokalen Denkund Handlungszusammenhänge folgende Konzeptformulierungen» zu ermöglichen. Im Fokus seines Interesses stehen Muster des Denkens, Sprechens und Handelns. Mit einer anderen Schwerpunktsetzung interessiert sich Gropengießer (2007) vor allem für Verstehensprozesse und fasst metaphorische Konzepte als metaphorische Vorstellungen von Lernenden. Diese Vorstellungen nennt Gropengießer (2004) «Denkfiguren». Die Rekonstruktion von Denkfiguren bzw. metaphorischen Konzepten eröffnet in diesem Sinne die Chance, Lernende zu verstehen und die Differenzen und Gemeinsamkeiten zwischen fachlichen Vorstellungen und Lernendenvorstellungen zu erfassen.

Neben Metaphern sowie metaphorischen Konzepten wird zur begrifflichen Klärung weiterhin das Konstrukt der «Schemata» nach Schmitt (2017, 48 ff.) konkretisiert. Die Schemata können verstanden werden als «einfache und gestalthafte Erfahrungen, denen noch keine Bildqualität attestiert werden kann, die jedoch schon als basale Muster in der Wahrnehmung selbst zu finden sind» (Schmitt 2017, 51). Eine exemplarische Veranschaulichung bietet die Visualisierung des «Ursprung-PfadZiel-Schema[s]» (Schmitt 2017, 55) bzw. «path»-Schematas (Abb. 5.5). Dieses Schema kann als konkreter Quellbereich verstanden werden, welcher für Moser (2000, 3) mindestens die folgenden Aspekte umfasst: «a starting point, a final point and a direction» sowie der Aspekt, "that time passes as someone moves along the path». Körperlich erfahren wir diese Strukturelemente bereits im Kleinkindalter, so Schmitt $(2017,56)$, wenn wir uns von «einem Platz zum anderen bewegen». Beim Vergleich des Schemas (Abb. 5.5) mit dem zuvor skizzierten metaphorischen Konzept (Abb. 5.4) ist zu erkennen, dass das Schema zum einen als Grundmuster identifizierbar ist. Zum anderen wird dieses Schema um verschiedene Aspekte erweitert, konkretisiert und adaptiert wird, z. B. in der Aussage «paralleler Pfade» oder in dem Ausdruck «Karriereschritte». In dieser Abgrenzung von metaphorischen Konzepten werden Schemata für Schmitt $(2017,51)$ «als separate Kategorie zusammengefasst und als präverbale Schemata begriffen, die der Bildung metaphorischer Konzepte vorangehen». 


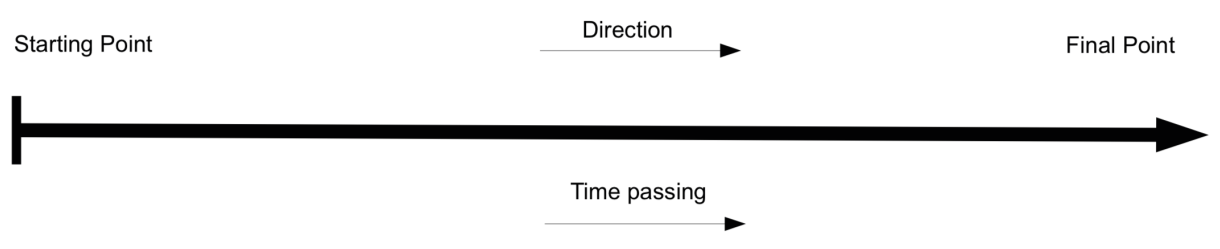

Related prozess: to move

Abb. 5.5: Schema of the metaphor source domain «path $\quad$ nach Moser $(2000,3)$.

Neben dem «Ursprung-Pfad-Ziel-Schema» skizziert Schmitt (2017, 53 ff.) in seinen Ausführungen sieben weitere aus seiner Perspektive wichtige Schemata: z. B. Gegenstandsschema, Substanzschema und Behälterschema. Die Frage, welchen Wert die Berücksichtigung von Schemata im Rahmen einer systematischen Metaphernanalyse als qualitative Forschungsmethodik besitzt, beantwortet Schmitt $(2017,55)$ mit den sich neu eröffnenden Möglichkeiten, metaphorische Redewendungen bzw. Einzelmetaphern überhaupt als solche zu erkennen:

«[S]o sind die Präpositionen 〈in> oder ‘außen〉 erst mit dem Wissen um ein Behälterschema als Hinweis auf eine Metaphorisierung zu deuten, wenn sie in Kontexten gebraucht werden, in denen eine räumliche Interpretation wenig Sinn ergibt, zum Beispiel: «in seinem Leben`. Hier wird das Abstraktum 〈Leben> als Behälter konstruiert, eine Interpretation, die freilich schlüssiger wird, wenn im gleichen Kontext davon gesprochen wird, dass dieses Leben (erfüllt) gewesen sei» (Schmitt 2017, 55).

Zusammenfassend lassen sich für die systematische Metaphernanalyse nach Schmitt $(2017,55)$ drei zentrale Begriffe bzw. Phänomene hervorheben:

1. «Konkrete metaphorische Redewendungen übertragen Bedeutungen von einem Quellbereich auf einen Zielbereich [...]». (Schmitt 2017, 51)

2. «Metaphorische Konzepte bündeln gleichsinnige Übertragungen mehrerer metaphorischer Redewendungen [...]» (ebd.).

3. «Schemata (wie z. B. das Gefäßschema) sind gegenstandsunabhängige, sehr allgemeine Muster der Wahrnehmung und kommen oft in Überschneidungen bei konkreten Redewendungen vor» (Schmitt 2017, 52).

Für die Erziehungswissenschaft als wissenschaftliche Praxis sowie für die Lehrerinnen- und Lehrerbildung als Lehrpraxis ist insbesondere das Konstrukt der metaphorischen Konzepte (Schmitt 2017) bzw. Denkfiguren (Gropengießer 2007) interessant. Diese Relevanz begründet Schmitt $(2017,52)$ dadurch, dass sich in ihnen «spezifische individuelle oder kulturelle Muster des Denkens, der Wahrnehmung, der Empfindung und des Handelns [bündeln]». Die weiteren mit diesem Konzept 
einhergehenden konzeptionellen Annahmen, die bereits in Ansätzen skizziert worden sind, lassen sich (ohne Anspruch auf Vollständigkeit) wie folgt zusammenfassen:

- Sprechen, Denken und Handeln beruhen auf dem gleichen Konzeptsystem. Die Konzepte sind weitgehend metaphorisch bzw. imaginativ strukturiert (z. B. Lakoff und Johnson 2014; Gropengießer 2004, 2007).

- Metaphorische Konzepte strukturieren unsere Wahrnehmung, unser Denken sowie unser Handeln (z. B. Lakoff und Johnson 2014).

- Abstrakte Sachverhalte und Begriffe (Zielbereich) können durch metaphorische Konzepte in bereits bekannten Sachverhalten und Begriffen (Quellbereich) erlebt und verstanden werden (z. B. Gropengießer 2004, 2007; Lakoff und Johnson 1990).

- Metaphorische Konzepte bzw. unser Konzeptsystem sind uns in der Regel nicht bewusst (z. B. Lakoff und Johnson 2014; Schmitt 2017).

- Über die Analyse von (Alltags-)Sprache wird es möglich, metaphorische Konzepte zu rekonstruieren (z. B. Lakoff und Johnson 2014; Gropengießer 2004 2007; Schmitt 2017).

- Metaphorische Konzepte bieten nur eine partielle Strukturierung eines Phänomens (z. B. Lakoff und Johnson 2014; Schmitt 2017; Gropengießer 2007).

- Für Abstrakte Sachverhalte können mehrere metaphorische Konzepte existieren (z. B. Lakoff und Johnson 2014; Gropengießer 2006, 2007) ${ }^{145}$.

- Metaphorische Konzepte lassen sich auf individueller, (sub-)kultureller und kollektiver Ebene rekonstruieren (z. B. Lakoff und Johnson 2014; Schmitt 2017; Gropengießer 2007) $)^{146}$.

Die zusammengestellten konzeptionellen Annahmen zum Konstrukt eines «metaphorischen Konzeptes» beschreiben, neben «Beliefs» (Kap. 5.3.1) und «Subjektiven Theorien» (Kap. 5.3.2), das dritte Forschungskonstrukt. Eine Positionierung erfolgt zum Abschluss des Kapitels 5.3.4.

\subsubsection{Schnittstellen zu Forschungskonstrukten und methodischen Zugängen}

In den vorherigen Kapiteln wurden verschiedene Schnittstellen von Metaphern zu den Konstrukten der Beliefs und Subjektiven Theorien auf begrifflicher und forschungsmethodischer Ebene skizziert. Für Gropengießer (2007) sind im Rahmen seiner Theorie des erfahrungsbasierten Verstehens Metaphern und metaphorische Konzepte insbesondere für die Aufarbeitung von Vorstellungen relevant. Dazu gehören sowohl die Vorstellungen von Lernenden zu einem spezifischen Gegenstand (Gropengießer 2007, 113 f.) als auch zur Aufarbeitung fachlicher Vorstellungen, wie

145 Lakoff und Johnson (2014) formulieren das auf kultureller Ebene und verdeutlichen es an unterschiedlichen metaphorischen Konzepten von Liebe. Gropengießer (2007) spricht in diesem Zusammenhang von «Denkweisen».

146 Hier unterscheiden sich die Autoren hinsichtlich ihrer Forschungsperspektiven und -gegenstände. 
sie in Form von Lehrbüchern und Fachtexten vorliegen (Gropengießer 2007, 114). In der Gegenüberstellung der unterschiedlichen metaphorischen Konzepte von Lernenden und verwendeten Metaphern in Fachtexten sieht Gropengießer (2007) ein Potenzial zur Gestaltung angemessener didaktischer Situationen. Wie im Rahmen der Diskussion Subjektiver Theorien bereits skizziert wurde, differenziert Gropengießer (1997, 2006) den Vorstellungsbegriff hinsichtlich unterschiedlicher Komplexitätsdimensionen. Die komplexeste Dimension ist anschlussfähig an den Begriff der Subjektiven Theorien. In der Diskussion von Vorstellungen über den Lehr- und Lernprozess verwendet Gropengießer (2004) den Begriff der Denkfiguren, welche konzeptionell anschlussfähig an das Konzept der metaphorischen Konzepte ist (Schmitt 2017). So erscheint der Geltungsbereich von metaphorischen Konzepten als Teilaspekt Subjektiver Theorien.

Eine umfangreiche Übersicht zu Anschlussstellen von Metaphern zu weiteren sozialwissenschaftlichen Begriffen und zur Diskussion der Frage, inwiefern Metaphern bzw. metaphorische Konzepte einen eigenständigen Begriff darstellen, bietet Schmitt $(2017,113 \text { ff. })^{147}$. Neben der Benennung einer Vielzahl empirischer Arbeiten widmet sich Schmitt (2017) dem Vergleich von Metaphern mit den sozialwissenschaftlichen Konzepten «soziale Deutungsmuster» (ebd., 117), «Habitus» (ebd., 133), «soziale Repräsentation» (ebd., 144), «Alltagspsychologie» (ebd., 148) sowie «Diskurs» (ebd., 161). Bei vergleichender Betrachtung der unterschiedlichen sozialwissenschaftlichen Konzepte hebt Schmitt $(2017,187)$ hervor, dass metaphorische Konzepte nicht «nur sozial geteilte Wissensformen ab[bilden], sondern auch individuelles Wissen». Zudem weist Schmitt $(2017,188)$ darauf hin, dass die benannten sozialwissenschaftlichen Begriffe und Konzepte durch «die jeweiligen theoretischen Vorannahmen das zu untersuchende Phänomen präfigurieren» und entsprechend begrenzen. Während metaphorische Konzepte bei Schmitt $(2017,188)$ diesbezüglich offener erscheinen, weist er darauf hin, dass sich Metaphernanalysen «derzeit auf eine bestimmte Klasse von sprachlichen Phänomenen beschränken» und entsprechend andere Grenzen aufweisen.

Für die empirische Untersuchung von metaphorischen Konzepten lassen sich sehr unterschiedliche Formen hinsichtlich der Auswahl des Datenmaterials, spezifischer Erhebungssituationen sowie Verfahren zur Auswertung identifizieren. Für die deutschsprachige sozialwissenschaftliche Forschung hat Schmitt (2017) in den letzten Jahren ein elaboriertes methodisches Konzept entworfen, welches bereits in verschiedenen empirischen Untersuchungen verwendet wurde. Für den Bereich der Lehrerinnen- und Lehrerbildung ist diesbezüglich das Vorgehen von Marsch (2009) ein interessantes Beispiel, um einen exemplarischen Einblick zu ermöglichen.

147 Für die sozialwissenschaftliche Forschung eröffnet Schmitt (2017, 113 ff.) eine Orientierung und Verortung da Lakoff und Johnson $(1980,2014)$ im Rahmen ihres meist rezipierten Werkes «Metaphors we live by» weitgehend auf Literaturbelege verzichten. 


\subsubsection{Orientierung für die Praxis Lehrerinnen- und Lehrerbildung}

Nach Marsch (2009, 106 ff.) können Metaphern als potenzielles Reflexionsinstrument für Lehrkräfte und Lehramtsstudierende verwendet werden. Im Rahmen der abschließenden Zusammenfassung ihrer Dissertation kommt Marsch $(2009,109)$ zu dem Schluss: «Metaphern können als Werkzeuge zur Reflexion und Veränderungen von Vorstellungen über das Lehren und Lernen genutzt werden». Dieses Potenzial sieht Marsch $(2009,106)$ «vor allem im Bereich der Lehrerfort- und -ausbildung» und führt verschiedene Überlegungen dazu auf Basis ihrer Ergebnisse vertiefend aus. So deutet Marsch $(2009,106)$ ihre Ergebnisse als Hinweis auf einen «Zusammenhang zwischen Metaphern, Vorstellungen und dem Handeln im Unterricht». Dabei schreibt Marsch (2009) Metaphern das Potenzial zu, neue Realitäten konstruieren zu können. Sie beschreibt in diesem Zusammenhang die Möglichkeiten von Metaphern, die Konzepte von Lehrerinnen und Lehrern artikulierbar und veränderbar zu machen. Zugleich weist Marsch $(2009,106)$ darauf hin, dass entsprechende Veränderungen von Vorstellungen und Konzepten «keinesfalls einfach» sind. Diese Aussage gilt nach Marsch $(2009,106)$ im theoretischen Rahmen der kognitiven Metapherntheorie nach Lakoff und Johnson (1980) und für die Subjektiven Theorien von Lehrerinnen und Lehrern. Diese Schwierigkeit begründet Marsch $(2009,107)$ wie folgt:

«Die Vorstellung von Lehrenden über das Lehren und Lernen sind noch schwieriger zu verändern als die von Schülern zu einem bestimmten Fachinhalt, da die Konzepte von Lehrern meist über viele Jahre entstanden sind und Lehren ein alltägliches Ereignis ist» (Marsch 2009, 107).

Dieses Ergebnis erscheint anschlussfähig an die bereits vorgestellten Erkenntnisse «Vorannahmen, Einstellungen bzw. Erwartungen» von Lehramtsstudierenden, die Blömeke (2004) unter dem Begriff der Beliefs zusammenfasst (Kap. 5.2). So wurden Beliefs von Blömeke $(2004,64)$ als erfahrungsbasiert und zugleich weitgehend «veränderungsresistent» beschrieben. Zum produktiven Umgang mit den Beliefs der Lehramtsstudierenden schlug Blömeke (2004) vor, an den jeweiligen Vorstellungen anzuknüpfen. Nach Marsch $(2009,106)$ erscheinen Metaphern dafür einen möglichen Ansatzpunkt zu bieten:

«Metaphern haben das Potential, Veränderungen der Vorstellungen vom Lehren und Lernen und des unterrichtlichen Handelns zu initiieren. Wenn wir anfangen, uns unserer Vorstellungen und den Erfahrungen, auf denen sie aufbauen, mithilfe von Metaphern bewusst zu werden, ist dies ein erster Schritt im Prozess zur Veränderung des dahinterstehenden Konzeptsystems» (Marsch 2009, 106). 
In der Diskussion der Relevanz ihrer Ergebnisse für das Lehramtsstudium vertritt Marsch $(2009,106)$ u. a. die Position: «Die Vorstellungen, die Lehramtsstudierende vom Lehren und Lernen haben, sowie die daraus resultierenden Handlungsorientierungen für den Unterricht sollten zu Themen der Ausbildung gemacht werden». Diese Überlegungen scheinen anknüpfungsfähig an die verschiedenen Positionen zur reflexiven Lehrerinnen- und Lehrerbildung, wie sie von Wildt (2013) skizziert worden sind (Kap. 5.2.3).

\subsubsection{Positionierung}

In den vorangegangenen Ausführungen wurden zunächst Subjektive Theorien und (educational) Beliefs als zwei Forschungskonstrukte skizziert, welche insbesondere im Bereich der Lehrerinnen- und Lehrerbildung verbreitet sind (Kap. 5.3.1 5.3.2). Anschließend wurde mit dem Begriff des metaphorischen Konzeptes ein weiteres Konstrukt skizziert, welches verschiedene Anschlussstellen und Überschneidungen zu Subjektiven Theorien sowie Überzeugungen aufweist (Kap. 5.3.3). Bei vergleichender Betrachtung der skizzierten Definitionsmerkmale werden verschiedene Gemeinsamkeiten und konzeptionelle Anschlussstellen erkennbar. Diese Gemeinsamkeiten werden im Folgenden hinsichtlich dreier Aspekte verdeutlicht: (1.) Erfahrungsfundierung, (2.) Funktion als Wahrnehmungsfilter sowie (3.) begrenzte Zugänglichkeit bzw. Bewusstseinsfähigkeit.

Der Begriff «Erfahrungsfundierung» wird als erste Gemeinsamkeit der drei unterschiedlichen Konstrukte gesehen und markiert die Annahme, dass rekonstruierbare Überzeugungen, Subjektive Theorien und metaphorische Konzepte jeweils auf individuelle Erfahrungen zurückzuführen sind. Blömeke $(2004,64)$ trifft in diesem Zusammenhang die Aussage, dass Beliefs «erfahrungsbasiert» sind. So beginnen Lehramtsstudierende nach Blömeke (2004) und Kane et al. (2002) ihr Lehramtsstudium mit eigenen Vorstellungen bzw. Beliefs über «schul- und unterrichtsrelevante Aspekte» (Blömeke 2004, 64). Die Entstehung entsprechender Überzeugungen kann, so Blömeke (2004), u. a. sozialisatorisch erklärt werden (Kap 5.3.1). Subjektive Theorien stellt Dann $(1989,248)$ als «relativ stabile kognitive Strukturen (mentale Repräsentationen) dar, die gleichwohl durch Erfahrung veränderbar sind». Die Möglichkeit zur Veränderung Subjektiver Theorien auf Basis von Erfahrungen erscheint anknüpfungsfähig zum gewählten Begriff «Erfahrungsfundierung». Zudem skizziert Dann $(1989,248)$ eine Handlungssituation von Lehrerinnen und Lehrern, bei diese auf unterschiedliche «Wissensbestände» zurückgreifen. Erworben wurde dieses Wissen, so Dann $(1989,248)$, «nur z. T. in der formalen Ausbildung [...], z.T. auch schon vorher in der eigenen Schulzeit und zum großen Teil erst durch die eigene mehr oder weniger reflektierte Praxis» (vgl. Kap 5.3.2). Für das Konstrukt metaphorischer Konzepte formuliert Schmitt (2017) in diesem Zusammenhang: «Menschen 
verstehen Unvertrautes dadurch, dass sie die Muster einfacher, älterer, individueller und sprachlich überlieferter kollektiver Erfahrungen auf neue Phänomene übertragen» (Schmitt 2017, 6). In Adaption der kognitiven Metapherntheorie zeigt Schmitt $(2017,6)$ im Vergleich mit sozialwissenschaftlichen Konstrukten, dass «metaphorische Konzepte» als «spezifische Rekonstruktion von Erfahrungsmustern» verstanden werden können (Kap 5.3.3).

Eine zweite Gemeinsamkeit der drei Konstrukte wurde mit dem Begriff «Wahrnehmungsfilter» beschrieben. Der Begriff «Wahrnehmungsfilter» knüpft an die Formulierung von Blömeke et al. (2003) sowie an Pajares (1992) an (Kap. 5.2.2.2). Für das Konstrukt der Beliefs wird die Annahme formuliert, dass unsere Überzeugungen in spezifischer Weise unsere Wahrnehmung sowie unser Denken definieren, verzerren bzw. formen. Pajares $(1992,325)$ beschreibt die Wirkung von Überzeugungen wie ein Filter, durch den neue Phänomene interpretiert werden. Eine ähnliche Formulierung für diese konzeptionelle Eigenschaft findet sich auch für die Konstrukte der Subjektiven Theorien sowie für metaphorische Konzepte. So kann u. a. nach Lakoff und Johnson (2014) davon ausgegangen werden, dass metaphorische Konzepte unsere Wahrnehmung, unser Denken sowie unser Handeln strukturieren (Kap 5.3.3). Dann $(1989,249)$ beschreibt diese Eigenschaft von Subjektiven Theorien wie folgt:

«Analog zu wissenschaftlichen Theorien erfüllen Subjektive Theorien die Funktionen (a) der Situationsdefinition i.S. einer Realitätskonstituierung; (b) der nachträglichen Erklärung (und oft der Rechtfertigung) eingetretener Ereignisse; (c) der Vorhersage (oder auch nur der Erwartung) künftiger Ereignisse; (d) der Generierung von Handlungsentwürfen oder Handlungsempfehlungen zur Herbeiführung erwünschter oder zur Vermeidung unerwünschter Ereignisse» (Dann 1999, 249).

Eine dritte konzeptionelle Gemeinsamkeit der drei Konstrukte lässt sich hinsichtlich ihrer begrenzten Zugänglichkeit bzw. Bewusstseinsfähigkeit markieren. Subjekte Theorien sind nach Dann $(1989,249)$ «teilweise implizit». Beispiele für Dann (1989, 249) sind «nichtbewußtseinsfahige (sic) Selbstverständlichkeiten oder unreflektierte Überzeugungen». Unter spezifischen Bedingungen, so Dann (1989, 249), sind Subjektive Theorien «teilweise aber dem Bewußtsein (sic) des Handelnden zugänglich, so daß (sic) er darüber berichten kann». Interessant an dieser Formulierung ist, dass Dann $(1989,249)$ den Begriff «Überzeugungen» als beispielhafte Veranschaulichung von impliziten Subjektiven Theorien verwendet. Ferner nennen Baumert und Kunter $(2006,497)$ eine ähnliche Definition für Beliefs bzw. für «pädagogisch relevante Überzeugungen als implizite oder explizite, subjektiv für wahr gehaltene Konzeptionen, welche die Wahrnehmung der Umwelt und das Handeln beeinflussen». Sowohl Subjektive Theorien als auch Überzeugungen sind gemäss den vorherigen Definitionen nur teilweise bewusst bzw. können nur partiell unter spezifischen 
Rahmenbedingungen explizit versprachlicht werden. Metaphorische Konzepte sowie das zugehörige Konzeptsystem sind nach Lakoff und Johnson (2014) und Schmitt (2017) in der Regel nicht bewusst, können aber durch die Analyse der Sprache rekonstruiert werden. Zudem gehen weitere Autorinnen und Autoren wie Saban et al. (2007) und Ryter Krebs (2008) davon aus, dass die Formulierung von Metaphern helfen kann, implizites Wissen und Überzeugungen explizieren zu können (Kap 5.3.3).

Auf Basis der skizzierten konzeptionellen Gemeinsamkeiten wurde sich für die Planung und Durchführung des Projektes entschieden, die Auseinandersetzung mit Metaphern zu vertiefen. So ist die Rekonstruktion von metaphorischen Konzepten zum einen anschlussfähig an die Diskussionen zum Diskurs über Beliefs und Subjektive Theorien. Zum anderen erscheint das Potenzial, Reflexionsprozesse anregen zu können, anknüpfungsfähig an die skizzierten Leitbilder der Lehrerinnen- und Lehrerbildung (Kap. 5.2.1). Wenn die Artikulation von Metaphern einen Zugang zu den Erfahrungen der Studierenden eröffnet, bietet sich im Sinne von Wildt (2003) die Möglichkeit, eine Relationierung von theoretischem Wissen und individuellen Erfahrungen zu unterstützen (Kap. 5.2.3). Zur Beantwortung der Frage, inwiefern die Artikulation und Analyse von Metaphern einen potenziellen Zugang zu den Erfahrungen und Überzeugungen der Studierenden eröffnen kann, werden in den zwei folgenden Kapiteln theoretische sowie empirische Erkenntnisse diskutiert (Kap. 5.4, 5.5).

\subsection{Metaphern in der Lehrerinnen- und Lehrerbildung}

In Anknüpfung an die Ausführungen zur theoretischen Verortung zwischen Subjektiven Theorien und Überzeugungen wurden Metaphern bzw. metaphorische Konzepte als möglicher Zugang zu erfahrungsbasierten Vorstellungen bzw. zum Denken und Sprechen diskutiert. Zur Erweiterung der begrifflich-theoretischen Auseinandersetzung wird im Folgenden eine stärker gegenstandsbezogene Auseinandersetzung mit Metaphern als Möglichkeit zur Reflexion von erfahrungsbasierten Vorstellungen von Lehrkräften und Lehramtsstudierenden in Lernsituation sowie deren wissenschaftlichen Untersuchung dargelegt. Dies erfolgt hinsichtlich der drei folgenden Schwerpunkte: Denk- und Sprachfiguren zum Lehr- und Lernprozess (Kap. 5.4.1), Metaphern als Datenquellen und Analyseobjekt (Kap. 5.4.2) und Reflexionsanlässe durch Metaphern (Kap. 5.4.3).

\subsubsection{Denk- und Sprachfiguren zum Lehr- und Lernprozess}

Eine Übersicht über deutschsprachige metaphorische Denk- und Sprachfiguren zum Lehr- und Lernprozess entwickelt und diskutiert Gropengießer (2004) im Kontext der fachdidaktischen Lehrerinnen- und Lehrerbildung. Gropengießer $(2004,8)$ geht davon aus, dass ein «tragfähiges professionelles Wissen über Lehr-Lernprozesse 
[...] eine notwendige Bedingung für eine professionell-reflexive Grundhaltung von Lehrpersonen» darstellt. Zur "Aneignung und Entwicklung professionellen Willens», so Gropengießer $(2004,8)$ weiter, bedarf es einer Berücksichtigung des Vorwissens bzw. der Vorstellungen der Lernenden. In der Darstellung verschiedener empirischer «Befunde zu Vorstellungen über Lehr-Lernprozesse» kommt Gropengießer (2004, 9 ff.) zu der Erkenntnis, dass die jeweiligen Befragten ihre Vorstellungen zum Lehren und Lernen überwiegend metaphorisch ausgedrückten. Zugleich zeigt sich diese Erkenntnis auch in Betrachtung pädagogisch-psychologischer Fachtexte zum Lehren und Lernen: «Der Lehr-Lernprozess wird weitgehend metaphorisch dargestellt» (Gropengießer 2004, 12). Eine weitere Gemeinsamkeit von Lernenden und Wissenschaftlerinnen und Wissenschaftlern besteht für Gropengießer (2004) darin, dass diese über mehr als eine Vorstellung zum Lehr- und Lernprozess verfügen. Im Gegensatz zu wissenschaftlichen Vorstellungen erscheinen die Äusserungen bzw. Vorstellungen der befragten Personen zum Lehr- und Lernprozess aus «Beobachtersicht in vielen Fällen als widersprüchlich» (Gropengießer 2004, 11). Um Lernenden die eigenen Vorstellungen bewusst zu machen und bewusst zur Sprache zu bringen, skizziert Gropengießer $(2004,15)$ in seinem Beitrag verschiedene «Denkfiguren zum Lehr-Lernprozess». Diese Denkfiguren stellen eine Sammlung verschiedener metaphorischer Konzepte dar.

Im Rahmen seines Beitrages identifiziert Gropengießer $(2004,22)$ vielfältige Ausdrucksmöglichkeiten bzw. Bildbereiche mit denen bestimmte Facetten des Lehrens und Lernens zum Ausdruck gebracht werden können: Bildbereiche «der Weitergabe, der Fütterung, des Verkaufens, des Makelns, der Diplomatie, der Reise, des Wasserflusses, des Gärtnerns, des Bauens, des Töpferns, der Arbeit, des Kampfes und der Schau konnten identifiziert und belegt werden». Zur Darstellung der Denkfiguren bzw. metaphorischen Konzepte dieser Bildbereiche skizziert Gropengießer (2004) jeweils die partielle Übertragung vom Bildbereich auf das Lehren und Lernen. Zum Beleg eines Konzeptes werden eine Vielzahl von Aussagen aufgelistet, welche unterschiedlichen Datenquellen entstammen. Diese Datenquellen enthalten Aussagen von Lehrerinnen und Lehrern, Aussagen von Wissenschaftlerinnen und Wissenschaftler, konventionalisierte Metaphern unseres Wortschatzes sowie von Gropengießer (2004, 15) gesammelte «Redewendungen und Ausdrücke zum Lehr-Lernprozess». Die so ermittelten metaphorischen Konzepte sind in diesem Sinne nicht als individuelle metaphorische Konzepte, sondern als kollektive bzw. sozial geteilte metaphorische Konzepte zu verstehen. Exemplarisch sollen an dieser Stelle die metaphorischen Denkfiguren: (1) «Der Lehr-Lernprozess ist Weitergabe», (2) «der Lehr-Lernprozess ist eine Reise» sowie (3) «Der Lehr-Lernprozess ist Gärtnern» wiedergegeben werden.

Das metaphorische Konzept «Der Lehr-Lernprozess ist Weitergabe» von Gropengießer $(2004,16)$ kann als eine der einfachsten Denkfiguren beschrieben werden. Diese besteht aus den folgenden Elementen: Eine gebende Person steht für eine 
Lehrkraft und Lernende werden als nehmende Personen symbolisiert. Entsprechend wird das Geben als Lehren und das Nehmen als Lernen verstanden. Die Gabe steht in dieser Denkfigur nach Gropengießer (2004) für das Wissen. Dieses metaphorische Konzept belegt Gropengießer $(2004,16)$ mit den folgenden Aussagen:

«Sie besitzt Wissen oder verfügt über Informationen. Er gibt sein Wissen nicht heraus. Sie gibt ihre Vorstellungen preis. Jemand gibt sein Wissen weiter. Er versucht etwas rüber zu bringen und damit sein Wissen zu übertragen. Das muss man denen beibringen. Jemand nimmt das Wissen oder den Lernstoff auf. Wir haben das schon durchgenommen. Sie fasst es gut auf. Jetzt hab' ich's. Ich hab's gepackt. Er hat's begriffen. Neue Vorstellungen kann man dort nicht erlangen. Wer alles fassen soll, wird nichts behalten» (Gropengießer 2004, 16; Hervorhebung im Original).

Ein weiteres metaphorisches Konzept beschreibt Gropengießer $(2004,18)$ als "der Lehr-Lernprozess ist eine Reise». Dabei werden die Reisenden als Lernende verstanden und die Reiseführerinnen und -führer bzw. Reisebegleiterinnen und -begleiter stehen für die Lehrkräfte. Die Fortbewegung steht in dieser Denkfigur für das Lernen. Im Vergleich zum vorherigen metaphorischen Konzept «Der Lehr-Lernprozess ist Weitergabe» lassen sich neben dem Bildbereich auch hinsichtlich des Zielbereichs verschiedene Unterschiede markieren. So finden sich keine Übertragungen für das Lehren sowie für das Wissen. Dafür wird mit dem Start der Reise der Lernanfang sowie mit dem Ziel der Reise der Lernerfolg die zeitliche Dimension des Lernens abgebildet. Ferner wird in dem Konzept von Gropengießer $(2004,18)$ der Weg als Lernprozess verstanden und Orientierung steht als Begriff für Lernhilfen. Dieses metaphorische Konzept belegt Gropengießer $(2004,18)$ u. a. mit den folgenden Aussagen:

«Lerner müssen dort abgeholt werden, wo sie stehen. Alle sollten mitgenommen werden. Sie können auch angestoßen werden, wenn sie nicht allein vorankommen. Lerner werden unterwiesen, sie werden auch angeleitet, sogar überwacht oder eng geführt, beispielsweise in einem Lehrgang, durch einen Leitfaden oder einen roten Faden, manches führt auch in die Irre, verleitet, ist eine falsche Spur oder Fährte. Lehrer führen oder orientieren ihre Schüler beim Lernen. Oft genügt auch eine Lernbegleitung. Wie weit sind wir gekommen? Einige finden den Lernweg selber, manche kommen nicht mit» (Gropengießer 2004, 18; Hervorhebung im Original).

Ein drittes exemplarisches metaphorisches Konzept wird von Gropengießer $(2004,19)$ als «der Lehr-Lernprozess ist Gärtnern» beschrieben. Dieses metaphorische Konzept beinhaltet in der Sammlung von Gropengießer $(2004,19)$ die meisten Abbildungen vom Bildbereich des Gartenbaus zum Zielbereich des Lehr- und Lernprozesses. Dabei versteht Gropengießer $(2004,19)$ die Gärtnerinnen und Gärtner als 
Lehrpersonen und das Blumenbeet als lernende Person. Im Vergleich zu den zwei vorherigen Konzepten werden Lernende in diesem Bild nicht als Menschen konzeptualisiert. Weite Elemente des Konzeptes sind Samen bzw. Pflänzlinge, die u. a. für Wissen und Gedanken sowie Wildwuchs und Unkraut welches metaphorisch falsches Wissen bedeutet, stehen. Verschiedene Aktivitäten des Gärtners werden von Gropengießer $(2004,19)$ auf die Aktivitäten der lehrenden Person projiziert: «Säen und Pflanzen = Lehren»; «Wässern und Düngen = Lernanregungen»; «Jäten = radikale Korrektur»; «Beschneiden, Stutzen = Korrektur». Die Ernte steht für das Lernergebnis im Rahmen dieses metaphorischen Konzeptes. Dieses metaphorische Konzept belegt Gropengießer $(2004,18)$ u. a. mit den folgenden Aussagen:

«Lehrer werden in der Pflanzschule des Geistes herangezogen. Dort wird der Boden bereitet für die geistige Entwicklung. Es wird die Saat gelegt für ihre spätere geistige Entwicklung. Das Wissen verbreitet sich schnell. Der Gedanke konnte eingepflanzt werden. Die Ideen fallen auf einen fruchtbaren Boden. Damit wird fruchtbares Lernen möglich. Oft kommt es zum Wachstum des Wissens. Einige Fähigkeiten müssen kultiviert werden. Sein Wissen ist ein zartes Pflänzchen, welches gehegt und gepflegt werden muss. Wachsen lassen, heißt die Devise [...]» (Gropengießer 2004, 19; Hervorhebung im Original).

Mit den drei skizzierten Denkfiguren bzw. metaphorischen Konzepten kann ein erster gegenstandsbezogener Einblick eröffnet werden, inwiefern mit Metaphern eine Untersuchung und Reflexion des Sprechens und Denkens über Lehren und Lernen erfolgen kann. Während Gropengießer (2004) sein forschungsmethodisches Vorgehen nur knapp skizziert, diskutiert er die Möglichkeiten entsprechender Denkfiguren für die Lehrerinnen- und Lehrerbildung. So weist Gropengießer $(2004,22)$ zum einen darauf hin, dass sich bestimmte metaphorische Konzepte besser eignen als andere, um bestimmte Facetten des Unterrichts zu beschreiben. Ein «nicht-direktives Verständnis des Lehr-Lernprozesses» erscheint Gropengießer $(2004,22)$ besser denk- und beschreibbar mit der Denkfigur der Diplomatie als mit der Denkfigur des Töpferns bzw. Formens. Den Nutzen der Auseinandersetzung mit entsprechenden metaphorischen Konzepten sieht Gropengießer $(2004,22)$ auf unterschiedlichen Ebenen:

- «Die Denkfiguren [...] können explizit bewusst machen, in welcher Weise wir in bestimmten Situationen über den Lehr-Lernprozess sprechen und denken» (Gropengießer 2004, 22). In diesem Sinne kann die Sammlung der Denkfiguren als Orientierungs- bzw. Selbstreflexionshilfe verstanden werden, um zum Nachdenken über die eigenen (impliziten) Vorstellungen und Überzeugungen anzuregen bzw. sich diesen bewusst zu werden.

- «Die vorliegende Sammlung von Denkfiguren bietet die Möglichkeit, die eigene Lehr-Lernpraxis und die Anderer zur Sprache zu bringen, indem verschiedene Ausdrucksmöglichkeiten gewählt werden können» (Gropengießer 2004, 22). Die 
Artikulation der eigenen Erfahrungen beinhaltet in Anlehnung an Jörissen und Marotzki (2009) bereits ein reflexives Moment. Wenn die Wahl der Ausdrucksmöglichkeiten bewusst erfolgt, erweitern sich zugleich die Möglichkeiten zur Selbstreflexion sowie die Chancen, die Perspektiven anderen nachvollziehen und diskutieren zu können.

- «Die Denkfiguren können als Grundlage zur kritischen Reflexion über das Denken und Handeln im Zusammenhang mit dem Lehr-Lernprozess dienen. Sie können dabei auch kreativ in wenig gebräuchliche Bereiche einer Metapher hinein erweitert werden und damit Ausgangspunkte für neue Wege des Denkens in diesem Bereich sein» (Gropengießer 2004, 22).

Während die kreative Ausgestaltung der Metaphern sowohl auf Grenzen und weitere Möglichkeiten hinweisen kann, lässt Gropengießer (2004) offen, nach welchen Kriterien eine kritische Reflexion erfolgen kann. Didaktische Modelle (bzw. deren metaphorische Konzepte) können in diesem Zusammenhang hilfreiche Kriterien zur Reflexion bieten. So könnten die Denkfiguren auch im Sinne von Wildt (2005) als Relationierung von Subjektiven Theorien und wissenschaftlichen Theorien verstanden werden (Kap. 5.2.3).

\subsubsection{Metaphern als Datenquelle und Analyseobjekt}

Metaphern von Lehrkräften und Lehramtsstudierenden stehen im Fokus zahlreicher englischsprachiger Studien. Über die Erhebung und Analyse von Metaphern wird u. a. das Ziel verfolgt, Aussagen über Beliefs (z. B. Poom-Valickis et al. 2012; Kalra und Baveja 2012; de Guerrero und Villamil 2000, 2002) sowie über Subjektive Theorien und Vorstellungen ${ }^{148}$ (z. B. Marsch 2009; Saban et al. 2007; Koc 2013; Kalra und Baveja 2012; Martinez et al. 2001) von Lehrkräften und Lehramtsstudierenden zu treffen. Zudem finden sich vielfältige Bezüge zur kognitiven Metapherntheorie (z. B. Kalra und Baveja 2012; Koc 2013; Thomas und Beauchamp 2011; Marsch 2009; de Guerrero und Villamil 2000, 2002). Um einen ersten Einblick in entsprechende empirische Verfahren zu eröffnen, werden im Folgenden zwei exemplarische Erhebungsverfahren skizziert: (1.) Aufgabenstellungen im Rahmen von Lehrveranstaltungen sowie (2.) Interviews mit Lehrkräften und Lehramtsstudierenden.

Exemplarische Studien zur Erhebung von Metaphern in Form Aufgabenstellungen liefern u. a. de Guerrero und Villamil (2000, 2002); Poom-Valickis et al. (2012); Arslan und Karatas (2015) sowie Saban et al. (2007). Gemeinsam ist diesen Studien jeweils die Aufgabenstellung für Studierende bzw. Lehrerinnen und Lehrern. Diese werden im Rahmen von Lehrveranstaltungen und Workshops dazu aufgefordert, eine

148 Die Zusammenfassung von Subjektiven Theorien erfolgt hier in Anlehnung an das Komplexitätsschema von Gropengießer (1997). 
Metapher bzw. einen expliziten Vergleich in der Form «Ein Lehrer ist wie ..., weil ...» zu formulieren. Die Einbindung dieser Aufgabenstellung in das didaktische Konzept der Veranstaltung sowie die gegebene Bearbeitungszeit variieren hinsichtlich der verschiedenen Untersuchungen bzw. werden nicht explizit formuliert. Im Rahmen der Untersuchung von Saban et al. (2007) erhielten die Studierenden beispielsweise 45 Minuten Zeit zur Entwicklung einer Metapher. Bei Arslan und Karatas $(2015,1477)$ betrug die Bearbeitungszeit 30 Minuten und es mussten zwei Metaphern entwickelt werden. Ferner skizzieren de Guerrero und Villamil $(2000,2002)$, in welcher Weise die Aufgabenstellung ihres Workshopkonzeptes die Teilnehmerinnen und Teilnehmer zur Reflexion ihrer Überzeugungen anregen soll. Bei Saban et al. (2007), Arslan und Karatas (2015) und Poom-Valickis et al. (2012) werden die so entstandenen Metaphern vor allem als Daten ihrer Untersuchung diskutiert. Die von den benannten Autorinnen und Autoren verwendete Aufgabenstellung findet sich auch in weiteren Untersuchungen zu unterschiedlichen Zielbereichen. Bei Kobak und Taskin (2012) und Koc (2013) besteht die Aufgabenstellung darin, eine Metapher für Technologie zu entwickeln, bei Kalra und Baveja (2012) dienen die Begriffe «Knowledge, Learning and Learners» als Zielbereiche der Metaphern.

Zur Analyse der so gewonnenen Daten kommen in den verschiedenen Untersuchungen unterschiedliche Methoden zum Einsatz. Zum einen werden deduktive Verfahren verwendet, um die studentischen Metaphern auf Basis existierender Modelle zu verorten. Poom-Valickis et al. $(2012,235)$ verwenden beispielsweise ein Modell zur Lehrerinnen- und Lehreridentität und unterscheiden drei Dimensionen: (a) «the teacher as a subject expert», (b) «the teacher as a didactics expert» und (c) "the teacher as a pedagogical expert». Zum anderen werden induktive Verfahren eingesetzt, um konzeptuelle Metaphern bzw. übergeordnete Kategorien zu entwickeln, welche einen Überblick über einzelnen Metaphern der Studierenden eröffnen (z. B. Saban et al. 2007; Kalra und Baveja 2012; Arslan und Karatas 2015). So haben beispielsweise Saban et al. (2007) im Rahmen ihrer Lehrveranstaltungen explizite Metaphern und Vergleiche zur Beschreibung von Lehrkräften von 1142 Lehramtsstudierenden erhoben und in einem Verfahren mit mehreren Schritten induktiv ausgewertet. Auf dieser Basis entwickelten Saban et al. $(2007,128)$ zehn verschiedene konzeptuelle Kategorien, welche die zentralen Merkmale der Metaphern hervorheben. Die jeweiligen Kategorien wurden unterschiedlich häufig genannt und wurden von verschiedenen Metaphern bzw. Bildbereichen abstrahiert. Exemplarisch werden im Folgenden vier Kategorien skizziert, welche einen Einblick in die studentischen Metaphern ermöglichen.

- Die Kategorie «Teacher as knowledge provider (student as passive recipient of knowledge)» (Saban et al. 2007, 128) umfasst die Aussagen von 300 Studierenden in Form von 15 verschiedenen Metaphern bzw. Bildbereichen (z. B. «sun», «candle», «tree/fruit tree»). 
- «Teacher as molder/craftsperson (student as raw material)» (Saban et al. 2007, 129) umfasst die Aussagen von 277 Studierenden in Form von 17 verschiedenen Metaphern bzw. Bildbereichen (z. B. «sculptor», «painter», «constructor»).

- «Teacher as facilitator/scaffolder (student as constructor of knowledge)» (Saban et al. 2007, 131) umfasst die Aussagen von 113 Studierenden in Form von zehn verschiedenen Metaphern bzw. Bildbereichen (z. B. «compass», «lighthouse»).

- «Teacher as nurturer/cultivator (student as developing organism)» (Saban et al. $2007,131)$ umfasst die Aussagen von 77 Studierenden in Form von vier verschiedenen Metaphern bzw. Bildbereichen (z. B. «gardener», «farmer»).

Die Ergebnisse von Saban et al. (2007) eröffnen einen umfassenden Überblick, welche expliziten Metaphern und Vergleiche von Lehramtsstudierenden in der Türkei verwendet wurden. Dabei lassen sich die konzeptuellen Kategorien eher als das Ergebnis einer strukturierenden Inhaltsanalyse nach Mayring (1990) verstehen, als eine Rekonstruktion individueller metaphorischer Konzepte. Die so gewonnenen Erkenntnisse eröffnen für die Gestaltung von Lehrveranstaltungen das Potenzial, sich über die artikulierbaren Metaphern zu informieren und die jeweiligen Implikationen der Metaphern für Diskussionsanlässe zu nutzen. Zugleich eröffnen Studien in dieser Form die Möglichkeit, kulturelle Unterschiede im Metapherngebrauch von Studierenden zu untersuchen. Die Grenzen dieser Erhebungsform sieht Schmitt $(2017,234)$ in der «handlungsfernen Erhebungssituation» Diese birgt die Gefahr, so Schmitt (2017, 234), «dass hier nicht unbedingt die handlungsrelevanten Metaphoriken generiert wurden». So wird auch die von Saban et al. $(2007,126)$ getroffene Annahme, die verwendete Aufgabenstellung schaffe die Möglichkeit für Studierende, «to make their implicit beliefs explicit», nicht vertiefend diskutiert.

Exemplarische Studien zur Erhebung von Metaphern in Form von Interviews liefern u. a. Marsch (2009); Marsch und Krüger (2007) sowie Thomas und Beauchamp (2011). Thomas und Beauchamp (2011) führten Interviews mit Studierenden zu unterschiedlichen Zeitpunkten ihres Studiums durch. Mithilfe leitfadengestützter Interviews als Methode der Datenerhebung untersuchte Marsch (2009) sowie Marsch und Krüger (2007) die Vorstellungen von Biologielehrerinnen und -lehrer zum Lehren und Lernen sowie zur Rolle als Lehrkraft. Die durchgeführten Interviews wurden mit einer qualitativen Inhaltsanalyse und einer Metaphernanalyse ausgewertet.

Zur Darstellung ihrer Ergebnisse unterscheiden Marsch und Krüger (2007) hinsichtlich «expliziter metaphorischer Denkfiguren» (259 ff.) sowie «impliziter metaphorischer Denkfiguren» (262 f.). Explizite metaphorische Denkfiguren ${ }^{149}$ versteht Marsch (2009) als metaphorische Redewendungen, die die Interviewten im abschliessenden Teil ihres Interviews bewusst wählten. Die explizit erfragten und von den

149 In der Beschreibung ihrer Ergebnisse verwendet Marsch (2009) zunächst voneinander abgegrenzte und differenzierte Begriffe teilweise synonym (z. B. Denkfigur und Metapher). 
Lehrerinnen und Lehrern gegebenen metaphorischen Aussagen wurden von Marsch und Krüger (2007) zum einen verschiedenen metaphorischen Denkfiguren zugeordnet $^{150}$. Zum anderen wurden die Aussagen aus der Projektperspektive «interpretiert und auf ihre Eignung zur Beschreibung von Lehren und Lernen unter konstruktivistischer Perspektive analysiert» (Marsch 2009, 61). Interessant an der Kombination der Auswertungsschritte ist, dass sichtbar wird, dass innerhalb des gleichen Bildbereiches der Fortbewegung sehr unterschiedliche individuelle metaphorische Konzepte artikuliert werden können. Dafür vergleicht Marsch $(2009,61)$ die zwei metaphorischen Konzepte «Lehrer als Bergführers» und «Lehrer als Schiff». Wenngleich sich in beiden Konzepten auf den Bildbereich der Fortbewegung zurückgegriffen wird, werden sie unterschiedlich ausgelegt. Zum einen wird Lehrerinnen und Lehrern «eine stark führende und dirigierende Rolle in Form eines Schiffs [zugeschrieben,] die den Schülern kaum Aktivität und eigene Entscheidungen ermöglicht» (Marsch 2009, 61). Zum anderen werden Lehrerinnen und Lehrer in der «Rolle eines Begleiters [beschrieben], der nur an schwierigen Stellen weiterhilft» (Marsch 2009, 61).

\begin{tabular}{|c|c|}
\hline DER LEHRER ALS BERGFÜHRER (L3) & DER LEHRER ALS SCHIFF (L4) \\
\hline & \\
\hline
\end{tabular}

Abb. 5.6: Visualisierung des Vergleichs unterschiedlicher Deutungen der Fortbewegungsmetaphorik von Marsch $(2009,61)$.

Interessant ist die Untersuchung von Marsch und Krüger (2007) darüber hinaus, da die Ergebnisse der qualitativen Auswertung der Fragen zu den Vorstellungen der Lehrenden mit den Ergebnissen der expliziten Metaphern der Lehrenden verglichen werden. Für Marsch $(2009,97)$ hat der Vergleich der Ergebnisse dieser unterschiedlichen Auswertungsmethoden gezeigt, «dass die Metaphern der befragten Biologielehrer mit ihren Vorstellungen vom Lehren und Lernen übereinstimmen». Beide Auswertungsmethoden, so die Schlussfolgerung von Marsch $(2009,98)$, «eignen sich also

150 Dabei bleibt offen welche Denkfiguren Marsch und Krüger (2007) zur Orientierung bzw. Analyse verwendeten. Vor dem Hintergrund der Bezugstheorien wird davon ausgegangen, dass sich diese auf Gropengießer (2004) beziehen. 
dazu, die Vorstellungswelt eines Individuums zu erschließen». Zugleich weist Marsch (2009) auf verschiedene Grenzen und Spannungsfelder hin:

- Zur Erweiterung der qualitativen Inhaltsanalyse in Form einer Triangulation weist Marsch $(2009,98)$ darauf hin, dass die «Analyse der expliziten Metaphern» das Potenzial bietet, Rückschlüsse auf die hinter den Metaphern stehende Vorstellungswelt zuzulassen». Zugleich zeigte sich im Rahmen eines Interviews von Marsch (2009, 101), dass nicht alle Teilnehmerinnen und Teilnehmer «gleichermaßen in der Lage sind, sich auf die Konstruktion einer individuellen Metapher einzulassen» (Marsch 2009, 98).

- Für die systematische Metaphernanalyse markiert Marsch $(2009,98)$ die Herausforderung, individuelle Vorstellungen über das Lehren und Lernen «durch die Analyse impliziter Metaphern» herauszuarbeiten. Diese Herausforderung begründet Marsch $(2009,98)$ mit der «starken Konventionalisierung und der Durchsetzung der Fachsprache mit Metaphern».

Vor diesem Hintergrund kommt Marsch $(2009,98)$ bei der Diskussion ihrer verwendeten Forschungsmethoden zu der Empfehlung: «Zur Erhebung, Analyse und Reflexion von individuellen Metaphern ist die explizite Vorgehensweise deshalb zu bevorzugen». Diese Empfehlung wurde in den Überlegungen zur Forschungsmethodik (Kap. 3.3.4) sowie zur Praxisgestaltung (Kap. 6.4.1) berücksichtigt.

\subsubsection{Reflexionsanlässe durch Metaphern}

Die Idee, mithilfe von Metaphern Reflexionsanlässe für Lehramtsstudierende im Rahmen von Lehrveranstaltungen zu schaffen, wird in zahlreichen der zuvor zitierten Studien markiert (z. B. Marsch 2009; Saban et al. 2007; Poom-Valickis et al. 2012; Martinez et al. 2001; de Guerrero und Villamil 2000, 2002). Dem Einsatz von Metaphern wird dabei u. a. das Potenzial zugesprochen, implizites Wissen, Vorstellungen und Überzeugen artikulieren und reflektieren zu können. Dafür werden Metaphern in unterschiedlicher Weise als Instrumente und Werkzeuge beschrieben, beispielsweise als «Reflexionswerkzeuge» (Marsch 2009, 108), «powerful cognitive tool» (Saban et al. 2007, 123) oder «as a tool, making implicit beliefs explicit» (Poom-Valickis et al. 2012, 234). Zugleich weist Marsch $(2009,107)$ darauf hin, dass kaum Erkenntnisse und Studien existieren, «wie eine solche Veränderung vonstattengeht». In Anknüpfung an Marsch (2009) wird diese Forschungslücke zunächst problematisiert. Anschliessend werden mit den Arbeiten von Martinez et al. (2001) sowie de Guerrero und Villamil $(2000,2002)$ Ansätze skizziert, wie Metaphern im Rahmen konkreter Lehrveranstaltungen und Workshops sowohl einen forschungsmethodischen als auch didaktischen Zweck erfüllen. 
Eine Gemeinsamkeit vieler Untersuchungen besteht mach Marsch $(2009,107)$ darin, dass viele Autorinnen und Autoren «großes Potential in der Nutzung von Metaphern als Reflexionswerkzeug» sehen und die Möglichkeiten «zur Veränderung von Vorstellungen zum Lehren und Lernen» markieren. Zugleich finden sich kaum empirische Untersuchungen, welche die Transformation von implizitem zu explizitem Wissen mithilfe von Metaphern untersuchen oder den Anregungsgehalt einer Auseinandersetzung mit Metaphern zur didaktischen Reflexion näher bestimmen. Über die Aufgabenstellung zur Formulierung eines expliziten Vergleichs in der Form «Ein Lehrer ist wie ...» geht ein Grossteil der zuvor zitierten Arbeiten nicht hinaus (z. B. Saban et al. 2007; Arslan und Karatas 2015). Die mit dieser Aufgabenstellung verbundene Annahme, Studierenden die Möglichkeit zu geben ihre impliziten Vorstellungen und Überzeugungen explizieren zu können, werden dabei kaum vertiefend diskutiert. Gleichwohl bleibt auch die Arbeit von Marsch (2009) hinsichtlich transformativer Potenziale für die Lehrerinnen- und Lehrerbildung - bedingt durch Fragestellung auf die Diskussion im Ausblick beschränkt. Zur konkreten Bestimmung wie eine gewünschte Veränderung anzuregen ist, zeigt Marsch $(2009,107)$ auf Basis ihrer Studie, «dass die Suche nach d e r besten Metapher zur Beschreibung konstruktivistisch orientierter Lernumgebungen nicht weiterführt». Für Marsch $(2009,107)$ ist es vielversprechender, auf die «die Explizierung der individuellen Metaphorik als Ansatzpunkt zur Entwicklung alternativer bzw. der Modifikation vorhandener Metaphern [zu setzen]». Für die Lehrerinnen- und Lehrerbildung schlussfolgert sie entsprechend, «dass Metaphern nicht vorgegeben werden können, sondern vom Individuum selbst konstruiert werden sollten» (ebd.).

Die Arbeiten von Martinez et al. (2001) sowie de Guerrero und Villamil (2000, 2002) stellen in Ansätzen zwei exemplarische Ausnahmen dar. Im Vergleich mit den vorherigen Arbeiten von Saban et al. (2007) und Marsch (2009) werden einerseits ähnliche Erkenntnisinteressen sichtbar ${ }^{151}$. Anderseits unterscheiden sich die Arbeiten hinsichtlich der Datenerhebung bzw. der Auseinandersetzung mit Metaphern im Rahmen von Lehrveranstaltungen für Lehramtsstudierende bzw. Workshops mit Lehrerinnen und Lehrern. Martinez et al. $(2001,968)$ beschreiben sowohl das Vorgehen ihrer Untersuchung als auch das Vorgehen in ihrer Lehre als Versuch,

«[...] to combine social learning approaches and individual learning. Phases of individual and collaborative reflection on metaphorical expressions of tacit knowledge alternate in our seminars in order to give our students access to their individual, implicit points of view as a condition to integrate explicit, scientific knowledge without distorting it. [...] The first part of the main study eliciting metaphors and subsequent discussions about their ideas of learning

151 Die Ziele sind ähnlich. Beispielsweise fragen de Guerrero und Villamil (2002) analog zu Saban et al. (2007): «How did the participants metaphorically conceptualize themselves as ESL teachers? What were the views of the ESL learner, teaching and learning entailed in the metaphors?» Auch die Arbeit von Martinez et al. (2001) ähnelt der Fragestellung und Auswertungsmethodik von Marsch (2009). 
started with a group discussion about the team members' individual metaphors of learning. The teams were obliged to record the results of their discussions in (written) formulations of those metaphors, which represented best the team's notion of learning» (Martinez et al. 2001, 968).

Einer ähnlichen Idee folgend, skizzieren de Guerrero und Villamil $(2000,2002)$ ein Workshopkonzept für Englischlehrende in Puerto Rico in mehreren Phasen. Zu Beginn des Workshops wurden die Teilnehmerinnen und Teilnehmer gebeten, ein Aufgabenformat in Form der vorherigen Untersuchungen auszufüllen, d.h. eine Metapher in Form eines expliziten Vergleichs («Ein Lehrer ist wie ...») zu formulieren. Im Verlauf des Workshops wurden die individuell entwickelten Metaphern auf Basis vorgegebener Fragestellungen in Gruppendiskussionen diskutiert und hinterfragt. Die Teilnehmerinnen und Teilnehmer wurden im Rahmen des Workshops dafür gebeten ihre Metaphern sowie die zugehörigen Notizen zu den folgenden Fragen auf individuellen Arbeitsblättern zu dokumentieren (Abb. 5.7). Die Arbeitsblätter dienten für de Guerrero und Villamil $(2000,2002)$ zudem als Datenmaterial zur Auswertung. Das Ziel der Auswertung war es u. a. die Metaphern zur identifizieren, die Englischlehrende verwenden, um ihre Rolle zur charakterisieren und sie darüber aufzuklären, welche theoretischen Annahmen über Lehren und Lernen sich in diesen Metaphern spiegeln.

\section{Workshop Worksheet}

I. [Write metaphor: An ESL teacher is like .... . $]^{4}$

II. [Identify the following elements in your metaphor: teacher, learner, teaching process, learning process, school environment, language, culture.]

III. [How does your metaphor reflect your daily practice?]

IV. [Identify the assumptions or theories underlying your metaphor.]

[How would you modify your metaphor? Is it necessary to change it?]

\begin{tabular}{|c|c|}
\hline Academic preparation: & bachelor's _, master's __, doctorate _, gr \\
\hline Teaching experience (years): & $1-5 \ldots, 6-10 \ldots, 11-15 \ldots, 15+$ \\
\hline $\begin{array}{l}\text { Current teaching level: } \\
\text { Gender: }\end{array}$ & $\begin{array}{l}\text { elementary _ } \ldots \text {, secondary __ higher education } \\
\text { male female }\end{array}$ \\
\hline
\end{tabular}

Abb. 5.7: Arbeitsblatt von de Guerrero und Villamil $(2000,351)$ zur Gestaltung eines Workshops.

Auf Basis 28 unterschiedlicher Metaphern der Teilnehmerinnen und Teilnehmer entwickelten de Guerrero und Villamil $(2000,2002)$ neun metaphorische Konzepte. Auffällig ist, dass am häufigsten, wie de Guerrero und Villamil $(2002,113)$ es nennen, klassische Rollenbilder verwendet wurden, z. B. Wissenslieferantin und 
Wissenslieferant, Erzieherin und Erzieher oder Künstlerin und Künstler. Die Rolle der Schülerinnen und Schüler umfasste ein breites Spektrum von aktiven Rollenbildern, z. B. Spielerin und Spieler, Musikerin und Musiker, Konstrukteurin und Konstrukteur, bis hin zu passiven Rollenbildern, z. B. Fernsehzuschauerin bzw. -zuschauer oder ein Stück Ton (Tab. 5.1).

\begin{tabular}{|l|l|l|l|}
\hline Role & Definition: The teacher & $\mathbf{n}$ & Examples \\
\hline $\begin{array}{l}\text { Cooperative } \\
\text { leader }\end{array}$ & $\begin{array}{l}\text {...guides and directs students, helping them } \\
\text { achieve goals; places herself or himself next } \\
\text { to students, not above as an authoritarian } \\
\text { figure; establishes an atmosphere of trust } \\
\text { in the classroom. }\end{array}$ & 6 & $\begin{array}{l}\text { coach, little leagues } \\
\text { coach, trail guide, mo- } \\
\text { vie/ theater director, } \\
\text { instrument of God, } \\
\text { symphony director }\end{array}$ \\
\hline $\begin{array}{l}\text { Provider of } \\
\text { knowledge }\end{array}$ & $\begin{array}{l}\text {...is the source and/ or conduit of language; } \\
\text { dispenses language knowledge to students. }\end{array}$ & 6 & $\begin{array}{l}\text { moon, wire in a thick } \\
\text { wall, television set, } \\
\text { sun, missile, tree full } \\
\text { of apples }\end{array}$ \\
\hline $\begin{array}{l}\text { Challenger/ } \\
\text { agent of } \\
\text { change }\end{array}$ & $\begin{array}{l}\text {...serves as a transformative agent in the stu- } \\
\text { dents' learning process by creating challenge, } \\
\text { bringing about change, and procuring oppor- } \\
\text { tunities for learning. }\end{array}$ & 6 & $\begin{array}{l}\text { snag in the river, } \\
\text { window to the world, } \\
\text { bullfighter, lion tamer, } \\
\text { gateway to the future, } \\
\text { shooting star }\end{array}$ \\
\hline
\end{tabular}

Tab. 5.1: Exemplarische Auswahl der konzeptuellen Kategorien von de Guerrero und Villamil (2000, 344).

Die Analyse der theoretischen Annahmen der Teilnehmerinnen und Teilnehmer zeigten de Guerrero und Villamil $(2002,114)$, dass diese in der Lage waren, von den jeweiligen Metaphern zu abstrahieren und den Metaphern zugrundeliegende Prinzipien zu formulieren. Die Qualität dieser Reflexionsleistung bezeichnen de Guerrero und Villamil $(2002,114)$ als «moderate level of abstraction». Typische Beispiele sind Aussagen wie: «l believe in acquisition by exposure), (positive affect is necessary), (students) prior knowledge can hinder or enhance learning), and sstudents cannot be forced to learn English»» (de Guerrero und Villamil 2002, 114). Explizite Verweise auf theoretische Ansätze oder Modelle konnten die Teilnehmerinnen und Teilnehmer hingegen nicht bzw. kaum formulieren. Lediglich ein fachspezifisches Modell wurde von verschiedenen Teilnehmerinnen und Teilnehmern genannt.

In Betrachtung der impliziten und expliziten Metaphern sowie deren Begründungen bietet die Untersuchung für de Guerrero und Villamil $(2002,114)$ ein Mass zur Beurteilung, inwieweit sich die Teilnehmerinnen und Teilnehmer die verschiedenen Ansätze zur Vermittlung von Zweit- und Fremdsprache angeeignet hatten. Sie betonen dabei, dass ihre Untersuchung Einsichten darüber eröffnet, wie Lehrende über ihre Rolle als Lehrkräfte sprechen. Aussagen über das Handeln können durch die Untersuchung nicht gemacht werden. Darüber hinaus betrachten de Guerrero und Villamil $(2002,113)$ ihre Ergebnisse als Einsichten in Metaphern und metaphorische 
Konzepte, die im Bewusstsein der Lehrerinnen und Lehrern im Vordergrund stehen und für die Externalisierung leicht zugänglich sind. Für de Guerrero und Villamil $(2002,113)$ eröffnen sich vor diesem Hintergrund Diskussionsmöglichkeiten der Frage, inwieweit der mentale Rahmen von ESL-Lehrerinnen und Lehrern soziokulturell gestaltet und von populären metaphorischen Überzeugungen beeinflusst wird.

\subsection{Metaphern in weiteren pädagogischen Kontexten}

Aktuelle Literaturübersichten zur Bedeutung von Metaphern in der Erziehungswissenschaft und der Pädagogik sowie zu den Perspektiven und zum Stand der erziehungswissenschaftlichen Metaphernforschung wurden in den letzten Jahren von Gansen (2010) und Schmitt (2017) vorgelegt. Beiden Arbeiten gemeinsam ist, dass sie sich an den zentralen Annahmen der kognitiven Metapherntheorie orientieren (Lakoff und Johnson 1990, 2014), diese kritisch diskutieren und für ihre jeweiligen Zielsetzungen weiterentwickeln. Der Blick auf die pädagogische und erziehungswissenschaftliche Metaphernforschung erfolgt dabei jeweils mit anderen Schwerpunkten $^{152}$. Auf Basis dieser Arbeiten wird ein skizzenhafter Überblick über verschiedene Forschungsfelder und -kontexte gegeben, in denen Metaphern in spezifischer Weise von Bedeutung sind. Dafür werden die folgenden Themen näher betrachtet: «Empirische Ansätze zur Metaphernforschung» (Kap. 5.5.2), «Metaphern beim pädagogischen Sprechen und Schreiben» (Kap. 5.5.1) und «Didaktische Funktionen von Metaphern in Bildungskontexten» (Kap. 5.5.3).

\subsubsection{Metaphern beim pädagogischen Sprechen und Schreiben}

Gansen (2010) und Schmitt (2017) zeigen in ihren narrativen Reviews auf, dass die Berufssprache und Wissenschaftssprache der Pädagogik bzw. der Erziehungswissenschaft durch vielfältige Metaphern geprägt ist. In Anlehnung an die kognitive Metapherntheorie (Kap. 5.3.3) vertreten sie die Position, dass die Sprache im Allgemeinen sowie in der Pädagogik im Besonderen nicht ohne metaphorische Redewendungen und Ausdrücke auskommt. Gansen (2010, 130) argumentiert beispielsweise, dass Grundbegriffe der Pädagogik wie «Bildung», «Erziehung» oder «Gestaltung» als «metaphorische, übertragene Ausdrücke» verstanden werden können. Diese Grundbegriffe haben, so Gansen $(2010,130)$, «ihre Wurzeln zum Teil in außerpädagogischen Bereichen und [erinnern] in ihrem bildlichen Gehalt an diverse Herkunftsbereiche».

152 Gansen $(2010,129)$ widmet sich im Rahmen seiner Dissertation u. a. dem Ziel, den deutschsprachigen Forschungsstand der «erziehungswissenschaftlichen Metaphernforschung» aufzuarbeiten und diesen hinsichtlich eines zeitgemässen Metaphernverständnisses zu diskutieren. Im Rahmen seiner Habilitation gibt Schmitt (2017) einen strukturierten Einblick in eine Vielzahl empirischer Studien hinsichtlich der jeweiligen Gegenstände entsprechender Forschungsarbeiten sowie der verwendeten forschungsmethodischen Zugänge. Im Fokus von Schmitt $(2017,257)$ steht der «erziehungswissenschaftliche Beitrag zu einer Methodik der systematischen Metaphernanalyse». 
Ohne diese Begriffe sowie Metaphern für diese Begriffe erscheinen die zentralen Gegenstände der Pädagogik kaum zugänglich. Die Relevanz von Metaphern kann hier in einem doppelten Sinne gesehen werden: Metaphern können zum einen zum Verständnis pädagogische Grundbegriffe beitragen. Zum anderen, so die Argumentation von Gansen $(2010,130)$, können die pädagogischen Grundbegriffe selbst einen metaphorischen Gehalt aufweisen. Für die Begriffe «Erziehung» und «Bildung» zeigt dies beispielsweise Meyer-Drawe (1999). Auch Schmitt $(2017,251)$ kommt zu dem Schluss, dass «die Pädagogik in ihrem lebensweltlichen Bezug ohne eine Fülle heterogener und alltäglicher Metaphern nicht auskommt». Die Sprache der Pädagogik umfasst in diesem Zusammenhang sowohl die Wissenschaftssprache der Erziehungswissenschaft, die Berufssprache professioneller Pädagoginnen und Pädagogen sowie die Alltags- bzw. Umgangssprache über Erziehungs- und Bildungszusammenhänge. Dieses umfassende Verständnis begründet sich durch die Besonderheit, wie Terhart $(1999,157)$ markiert, dass «die syntaktischen und semantischen Elemente der Umgangssprache, der Berufssprache und der Wissenschaftssprache nicht deutlich voneinander abgegrenzt sind, sondern gleichsam quer über alle Ebenen hinweg zirkulieren». Zur exemplarischen Veranschaulichung der eingangs formulierten Aussagen von Gansen (2010) und Schmitt (2017) werden im Folgenden exemplarische Erkenntnisse erziehungswissenschaftlicher Metaphernforschung skizziert, welche sich der Reflexion der sprachlichen Mittel der Erziehungswissenschaft widmen ${ }^{153}$.

Eine exemplarische Untersuchung zur Diskursgeschichte pädagogischer Metaphorik ist für Schmitt (2017) die Arbeit von Meyer-Drawe (1999). Meyer-Drawe (1999) widmet sich dem Ziel, den metaphorischen Gehalt der zwei pädagogischen Grundbegriffe «Erziehung» und «Bildung» näher zu bestimmen. Im Fokus ihrer Betrachtung stehen also «nicht Metaphern für «Bildung) und «Erziehung), [...] sondern das Metaphorische dieser Worte selbst soll in Erinnerung gerufen werden» (Meyer-Drawe 1999, 161). Damit unternimmt Meyer-Drawe $(1999,164)$ den Versuch, auf «abgesunkene Bedeutungen» hinzuweisen, welche wir zwar nicht mehr bemerken, die aber, so Meyer-Drawe $(1999,164)$, «unseren Begriffsgebrauch gleichwohl mitbestimmen». In Betrachtung der Diskursgeschichte lässt sich für den Erziehungsbegriff festhalten: «Für «Erziehung) gilt, daß (sic) sie sich nicht von Zucht trennen läßt (sic)» (MeyerDrawe 1999, 172). Unabhängig vom Kontext beinhaltet die Verwendung des Erziehungsbegriffs für Meyer-Drawe $(1999,172)$ die Bedeutung der «Unterordnung unter ein Allgemeines, und zwar zu Gunsten des übergeordneten Generellen». Da sich der Begriff nicht dazu eignet, «den Akt zu verleugnen», ist es aus der Sicht von (MeyerDrawe 1999, 172 f.) sinnvoller zu fragen, in welcher Weise Zucht in der Debatte über Erziehung jeweils thematisiert wird. Für den Bildungsbegriff hebt Meyer-Drawe

153 Das Metaphern dabei eine bedeutende Rolle spielen, zeigt das Themenheft der Zeitschrift für Pädagogik «Sprache der Erziehungswissenschaft» (Terhart 1999), in dem sich zwei von drei Beiträgen explizit Metaphern widmen (Peyer und Künzli 1999; Meyer-Drawe 1999). 
(1999, 173) die Bedeutung des «Schöpfungsmysteriums» hervor. Dieses Mysterium kann verständlich machen, warum - hinsichtlich der Bezugstheorien - «sehr unterschiedliche Reflexionen zum Bildungsbegriff [...] «umstandslos unter demselben Wort zur Diskussion gestellt werden können» (Meyer-Drawe 1999, 173).

« «ildung〉, die das Andere des Anderen als solches respektieren will, und «Bildung, die ungehemmte Selbsterschaffungsprozesse meint, beziehen sich beide auf grundsätzliche Unfaßlichkeiten (sic). [...] In beiden den heutigen Diskurs um den Bildungsbegriff bestimmenden Denkweisen ist, so gesehen, von einem Mysterium die Rede, vom Geheimnis des Anderen und vom Rätsel der Bedeutung» (Meyer-Drawe 1999, 173).

Meyer-Drawe $(1999,163)$ ging für ihre Metaphernanalyse davon aus, dass Metaphern - im Gegensatz zu mathematischen Formeln - «an unserer sinnlichen Existenz» teilhaben. Sprache weist in diesem Sinne eine grundsätzliche Metaphorik auf. Diese kann für Meyer-Drawe $(1999,163)$ an Begriffen wie «ver-stehen», "wahr-nehmen», «be-greifen» veranschaulicht werden. Zugleich weist Meyer-Drawe (1999) darauf hin, dass wir diese «leibliche Grundierung unserer Bezüge zur Welt» in unserer Sprache meist nicht mehr als Metaphern wahrnehmen und dass es einer besonderen Anstrengung bedarf, «sie hinter dem Schleier einer Abstraktion, die zur Denkgewohnheit geworden ist, zu entdecken» (ebd.). Mit dem skizzierten Verständnis von Metaphern lassen sich vielfältige Anknüpfungspunkte an die Grundannahmen der kognitiven Metapherntheorie erkennen (Kap. 5.3.3). Insbesondere die grundlegende Annahme der Analyse, dass die Explikation des metaphorischen Gehalts pädagogischer Begriffe dazu beizutragen kann, für die jeweils latenten Bedeutungen zu sensibilisieren, wird in den folgenden Überlegungen aufgegriffen.

Eine zweite exemplarische Untersuchung zur Diskursgeschichte pädagogischer Metaphorik ist die Dissertation von Guski (2007), welche von Gansen (2010) und Schmitt (2017) thematisiert wird. Statt den metaphorischen Gehalt pädagogischer Begriffe zu analysieren, widmet sich Guski (2007) dem Ziel, metaphorische Konzeptualisierungen «von Schule, schulischem Lernen und Lehren in pädagogischen Texten» historisch-systematisch zu erfassen. Auf Basis schulpädagogischer Texte «von Comenius bis zur Gegenwart» rekonstruiert Guski $(2007,143)$ zunächst «historisch konstante metaphorische Modelle von schulischem Lernen». Guski (2007) hebt neun verschiedene metaphorische Grundkonzepte hervor. Konzepte des schulischen Lernens umfassen u. a. die Metaphorik der «Fortbewegung auf einem Weg» (ebd., 144 ff.) und das organische Wachsen. Das schulische Lehren wird u. a. als "Stoffweitergabe» (ebd., 169 ff.) und als «Materialbearbeitung» (ebd., 173 ff.) konzeptualisiert. Darüber hinaus widmet sich Guski (2007) der Erarbeitung einer epochenspezifischen Metaphorik und unterscheidet metaphorische Konzepte hinsichtlich fünf unterschiedlicher 
«Epochen»: (1.) bei Comenius, (2.) zur Pädagogik der Aufklärung, (3.) im 19. Jahrhundert, (4.) Schulmetaphern um 1900 sowie (5.) Metaphern nach 1965.

Auf forschungsmethodischer Ebene beschreibt Schmitt (2017) die Arbeit als eine «von der kognitiven Linguistik inspirierten Dissertation». Für Gansen $(2010,146)$ werden die vielfältigen Quellen «historisch und diskursanalytisch aufgearbeitet». Guski (2007) diskutiert ihr Analysevorgehen mit Bezug zur «Kognitiven Metapherntheorie» (ebd., 469) sowie «Blending Theorie» (ebd., 471). Die inhaltlichen Ergebnisse der Arbeit bieten aufgrund ihrer Nähe zu Modulinhalten weitere Orientierungspunkte.

Als dritte exemplarische Untersuchung zur Sprache der Pädagogik wird im Folgenden die Analyse von Peyer und Künzli (1999) zu «Metaphern in der Didaktik» vorgestellt. Für Schmitt $(2017,237)$ ist das zentrale Ergebnis der Analyse, dass Peyer und Künzli (1999) für den deutschsprachigen Raum gezeigt haben, dass die Beschreibung «der Interaktion des Lehrens und Lernens nicht ohne Metaphern auskommt». Im Gegensatz zu den zwei historisch-orientierten Metaphernanalysen widmen sich Peyer und Künzli (1999) der Analyse von didaktischen Texten, welche aus ihrer Sicht für die Lehrerinnen- und Lehrerbildung in Deutschland repräsentativ sind. Peyer und Künzli $(1999,178)$ gehen davon aus, dass der «Bild- und Metapherngebrauch in der Didaktik zum einen Ausdruck ihres hohen, aber nur schwer zu bewältigenden und ungelösten Allgemeinheitsanspruches und zum anderen Indikator für ihre Zeit- und Herkunftsprägung ist». Anders als die vorherigen skizzierten Arbeiten fragen Peyer und Künzli $(1999,178)$ auf Basis von Texten sechs verschiedener Autorinnen und Autoren, «wie sich diese Spannung zwischen Allgemeinheitsanspruch und individueller Prägung durch Autor, Zeit- und Verwendungszusammenhang manifestiert». Dabei kommen sie in Abhängigkeit von den jeweiligen Autorinnen und Autoren zu deutlich unterschiedlichen Einschätzungen.

In der Analyse des Textes von Grell und Grell (1987) kommen Peyer und Künzli $(1999,186)$ zu der Einschätzung, dass die didaktischen Vorstellungen der Autorinnen und Autoren von «einem pragmatisch-praktizistischen Interesse und einem didaktischen Feindbild: dem fragend-entwickelnden Unterricht» dominiert werden. Dies wird für Peyer und Künzli $(1999,186)$ u. a. durch den expliziten Metapherngebrauch deutlich, in dem Grell und Grell (1987) die Position von Lehrerinnen und Lehrern im fragend-entwickelnden Unterrichtsgespräch als «Bettler» umschreiben, «die um Almosen der Schüler bitten und immer nur einzelne trockene Wörter bekommen, während die Schüler sich mit längeren Beiträgen vorsichtig oder vornehm zurückhalten, weil sie nicht sicher sein können, was der Lehrer will» (Grell und Grell 1987, 218 f.).

Dabei kann an diesem Zitat exemplarisch deutlich werden, was Peyer und Künzli $(1999,186)$ für die gesamte Arbeit kommentieren: Der verwendeten Bildsprache wird kaum «substantielle inhaltliche Geltung zuerkannt» und die jeweiligen 
metaphorischen Konzepte «lassen keinen Fokus und keine wiederkehrende Zentrierung der Argumentation erkennen». So wird auch das Bild des Bettlers nicht konsequent durchgehalten, wie an den Formulierungen «trockene Wörter», vorsichtiger oder vornehmer Zurückhaltung sowie Unsicherheit der Schülerinnen und Schüler zu erkennen ist. Für Grell und Grell (1987), so die Einschätzung von Peyer und Künzli (1999, 186), ist jedes Bild recht, «solange es seine rhetorische Wirkung zu tun verspricht, den Leser oder die Leserin anzusprechen, zu unterhalten oder abzuholen». Vor dem Hintergrund ihrer Fragestellung kommen Peyer und Künzli $(1999,186)$ zu der zusammenfassenden Einschätzung: «Statt einem theoretischen Allgemeinheitsanspruch signalisiert die Sprache von Grell und Grell (1987) einen allgemeinen Publizitätsanspruch und Kundenorientierung.»

In der sprachwissenschaftlichen Analyse von Roth $(1968,45)$ lassen sich nach Peyer und Künzli $(1999,187$ f.) deutlich konsequentere metaphorische Konzepte identifizieren, die im Gegensatz zu Grell und Grell (1987) deutlich anders entfaltet werden. So wird der Unterricht bei Roth (1968) nach Peyer und Künzli (1999, 187 f.) unter anderem mit dem Herkunftsbereich von «Frucht, Wachstum, Reifung und dem Geburtsprozess» verbunden, wie sie an dem folgenden Zitat veranschaulichen:

"[Gesinnung] muß (sic) herauswachsen aus dem liebenden Interesse am Gegenstand als eine Frucht des Geistigen, das in den Auseinandersetzungen während des Unterrichtsprozesses dem Gegenstand entbunden und in dem Kind erweckt wird. Liebendes Interesse [...] ist die treibende Kraft des schaffenden Lebens selbst, das nehmend und gebend an der Kultur teilnimmt. [...] Anknüpfungspunkte zwischen Kind und Gegenstand sind erregende und fruchtbare Momente im Unterrichtsprozeß (sic)» (Roth, 1968, 45).

Zugleich markieren Peyer und Künzli (1999, 187 f.) verschiedene Fragen, die sich bei Betrachtung der Bildsprache von Roth (1968) stellen, z. B. «ob der Lehrer nur Geburtshelfer sei oder auch Erzeuger». Auch die Grenzen der jeweiligen Bilder werden hinterfragt, z. B. «wird bei dieser Bildlichkeit nicht klar», so Peyer und Künzli (1999, 187 f.), «was genau seine Rolle ist, wenn der Unterrichtsprozeß einmal angefangen hat, denn dieser ist in seiner Natürlichkeit eigenständig». In der Gesamtbetrachtung der Ausführungen von Roth (1968) markieren Peyer und Künzli $(1999,188)$ zudem eine Spannung zwischen «Bildern von natürlichem Wachstum und von Beherrschung unheimlicher Kräfte voller Tükken». Für Peyer und Künzli $(1999,188)$ kann die Vielfalt der Bildsprache von Roth «als Ausdruck eines stark rhetorischen Interesses gedeutet werden, wobei mehr die Anschlußähigkeit und Wirksamkeit der Argumentation im Vordergrund steht, weniger eine konsistente Ideenfokussierung» (ebd.).

Als drittes Beispiel untersuchen Peyer und Künzli (1999, 188 f.) verschiedene Texte von Wolfgang Klafki $(1968,1991)$ Beim Vergleich mit den zwei vorherigen Beispielen ist die Sprache von Klafki für Peyer und Künzli $(1999,188)$ «beinahe unterkühlt». 
So verzichtet Klafki $(1968,1991)$ weitgehend auf die Verwendung expliziter Bilder wie sie von Grell und Grell (1987) sowie Roth (1968) skizziert werden. Stattdessen scheint Klafki nach Peyer und Künzli $(1999,189)$ «den metaphorischen Grundzug aller Sprache, wie sie in den 〈toten> Metaphern latent bleibt, zu nutzen». Beispielhaft wird die für Peyer und Künzli $(1999,188)$ an Verben wie «verflochten», «entfaltet» oder «fundiert» erkennbar. An den wenigen Textstellen bei denen explizite Bilder Verwendung finden, existieren nach Peyer und Künzli $(1999,188)$ «keine Konflikte in der Bildwelt»: «Methoden des Lehrens und Lernens müssen hier nicht nur als der jeweiligen Thematik dienende Lernformen und Lehrhilfen, sondern auch in ihrer Funktion als Anreger und Vermittler (oder auch als Begrenzungen sozialer Lernprozesse) durchdacht ... werden (ebd., S. 283). Welche ‘Anschauungen` sind geeignet, die auf das Wesen des jeweiligen Inhaltes, auf seine Struktur gerichtete Fragestellung in den Kindern zu erwecken, jene Fragestellung, die gleichsam den Motor des Unterrichtsverlaufs darstellen soll?» (Klafki 1968, 54).

Bei der Gesamtbetrachtung der Texte von Klafki $(1968,1991)$ fält es für Peyer und Künzli $(1999,188)$ auf, "wie wenig fachsprachlich terminologisch im engeren Sinne Klafkis Text geschrieben ist und wie sinnlich und plastisch seine Aussagen bei fast vollständigem Verzicht auf einen expliziten Metapherngebrauch doch formuliert sind.» Gleichwohl wird bei Peyer und Künzli $(1999,188)$ darauf verzichtet, mögliche metaphorische Konzepte zu rekonstruieren. So erscheint sich Klafki (1968) im Rahmen des exemplarischen Zitats unterschiedlicher Herkunftsbereiche zu bedienen. Während die Formulierung «Begrenzungen sozialer Lernprozesse» an ein BehälterSchema erinnert, lässt sich das «Wesen des jeweiligen Inhaltes» als Personifizierung der Inhalte deuten und die explizite Metaphorik vom «Motor» des Unterrichtsverlaufs entstammt einem maschinellen Herkunftsbereich.

Für die vorliegende Arbeit ist die Analyse auf zwei Ebenen interessant: Zum einen wird an den exemplarisch analysierten Texten deutlich, dass sich Didaktikerinnen und Didaktiker in unterschiedlicher Weise einer Bildsprache bedienen. Neben der von Schmitt $(2017)$ und Meyer-Drawe $(1999,163)$ formulierten Annahme der grundsätzlichen Metaphorik der Sprache, verwenden die verschiedenen Autorinnen und Autoren Metaphern mit zur Veranschaulichung und Zuspitzung ihrer Argumentation. Vor diesem Hintergrund kann die Analyse von Peyer und Künzli $(1999,188)$ dahingehend gewertet werden, dass «Metaphern nicht nur als rhetorischer Schmuck dienen, sondern ein wichtiges Instrument sind für unseren Umgang mit Wirklichkeit». Zum anderen lohnt es sich, Metaphern nicht nur im Sinne der kognitiven Metapherntheorie als kognitive Muster zu begreifen, sondern sich auch die verschiedenen rhetorischen und didaktischen Funktionen von Metaphern klarzumachen. In der (Selbst-) Reflexion der eigenen Wissenschaftssprache der Erziehungswissenschaft werden zudem unterschiedliche Analysemethoden sichtbar. Die Unterschiede zeigen sich im 
analysierten Material des zentralen Metaphernbegriffs bzw. der theoretischen Bezugspunkte sowie in der Konzeption des Forschungsgegenstandes der Analyse.

\subsubsection{Empirische Ansätze zur Metaphernforschung}

Mit der Darstellung der ausgewählten Beispiele zur Untersuchung von Metaphern in der pädagogischen Sprache (Kap. 5.5.1) wurden bereits unterschiedliche methodische Herangehensweisen. In diesen Untersuchungen wurden überwiegend wissenschaftliche Texte als Datenmaterial verwendet. Zudem existieren weitere empirische Ansätze, welche im deutschsprachigen Diskurs, so Gansen (2010, 160), eher vereinzelt zu finden sind. Auch Schmitt $(2017,226)$ markiert, dass in der Erziehungswissenschaft vor allem differenzierte Untersuchungen zur «Selbstreflexion der Disziplin» vorliegen. Zugleich gibt er einen umfassenden Einblick in die vielfältigen empirischen Ansätze und Zugänge der Metaphernforschung über den deutschsprachigen Diskurs hinaus. Schmitt (2017, 226 ff.) unterscheidet zwischen Ansätzen auf Basis der kognitiven Metapherntheorie und «empirische[n] metaphernanalytische[n] Arbeiten ohne Bezug zu Lakoff und Johnson» (Schmitt 2017, 243 ff.).

Für die vorliegende Arbeit wird im Folgenden ein Einblick in ausgewählte Forschungsprojekte gegeben, in denen Metaphern sowie metaphorische Konzepte zum Lernen in der Hochschule (Ryter Krebs 2008), zum Erleben von Schule (Geffert 2006) sowie zum Lernen in der Alltagssprache (Wiedenhöft 2014) rekonstruiert werden. Während sich die Forschungsarbeiten hinsichtlich der konkreten Forschungsgegenstände jeweils unterscheiden, wird in allen Arbeiten der Versuch unternommen, die Perspektive der jeweils Lernenden in den Blick zu nehmen. Dabei werden zugleich sehr unterschiedliche methodische Herangehensweisen erkennbar.

Eine exemplarische Untersuchung der «Alltagsvorstellungen des Lernens» ist für Schmitt $(2017,230)$ die systematische Metaphernanalyse mit dem Titel «Vom Reinziehen, Eintrichtern und Anbohren» von Wiedenhöft (2014). In der Aufarbeitung des Forschungsstandes markiert Wiedenhöft $(2014,24)$ das Fehlen einer systematischen Aufarbeitung «der zum Thema Lernen und Lehren gebräuchlichen Metaphern, um die Bandbreite dessen, was Konzepte des Lernens für Lernende, Lehrende und das zu erwerbende Wissen an unterschiedlichen Vorstellungen bereithalten kann, besser zu erfassen». Vor diesem Hintergrund widmet sich Wiedenhöft $(2014,24)$ dem Ziel, «ein möglichst breites Spektrum der in der Alltagssprache existierenden Metaphoriken aufzuzeigen». In Anlehnung an das von Schmitt (2003) entworfene Vorgehen zur systematischen Metaphernanalyse untersuchte Wiedenhöft $(2014,25)$ verschiedene "Quellen des alltäglichen Lebens»: Zeitungen und Zeitschriften (z. B. Die ZEIT, GEO oder DER SPIEGEL), die Radiosendung des Deutschlandfunks «Journal am Vormittag, Forum Pisa: 〈Wie und warum lernen Kinder»» sowie «Gespräche mit Teilnehmenden und Lehrenden einer beruflichen Weiterbildung». Im Rahmen ihrer Analyse arbeitet 
Wiedenhöft (2014, 29 ff.) 12 verschiedene metaphorische Konzepte zum Lernen in der Alltagssprache heraus. Die Strukturierung der Konzepte erfolgt hinsichtlich der identifizieren Quell- bzw. Bildbereiche. Dies zeigt sich an drei exemplarisch ausgewählten Konzepten von Wiedenhöft (2014): «Vom Einblicken und Durchschauen - Die Metaphorik des Sehens» (ebd., 29), «Vom Einflössen und Eintrichtern - Die Metaphorik des Behälters» (ebd., 33) sowie «Hegen und Pflegen - Die Metaphorik des Gärtners» (ebd., 40).

In der Diskussion ihrer herausgearbeiteten Konzepte weist Wiedenhöft (2014, 77) darauf hin, dass sich die auftretenden Bedeutungen innerhalb einer Metaphorik "durchaus unterscheiden können, schon ein einzelnes Konzept kann u.U. entgegengesetzte Sichtweise zulassen». Exemplarisch verweist Wiedenhöft $(2014,77)$ auf die Gärtner-Metaphorik: «dessen Aufgabengebiet ist zu breit gefächert [...], um daran eine bestimmte pädagogische Sichtweise festzumachen; vielmehr lassen sich eine große Anzahl verschiedener Auffassungen mit diesem Bild vereinbaren». Für Schmitt $(2017,230)$ ist die Breite der Datenerhebung dafür verantwortlich, dass die Autorin gezwungen ist, "die scheinbare Eindeutigkeit der gefundenen metaphorischen Konzepte zu relativieren». Zugleich stellt sich die Frage, inwiefern die von Wiedenhöft $(2014,24)$ herausgearbeiteten Konzepte eine notwendige Präzision aufweisen oder eher auf der Ebene von Schemata verbleiben, wie Schmitt (2017) es auch bei der Arbeit von Marsch (2009) problematisiert.

Für das vorliegende Forschungsprojekt ist die Arbeit von Wiedenhöft (2014) aus unterschiedlichen Gründen interessant. Inhaltlich bietet die herausgearbeitete Metaphorik eine mögliche Vergleichsfolie zur Analyse von metaphorischen Konzepten von Studierenden, ähnlich dem Vorgehen von Geffert (2006). Interessant sind dabei weniger die konventionellen Metaphernmodelle sondern, wie Ryter Krebs (2008, 214) markiert, deren individuelle Ausgestaltung durch die Studierenden. Ferner wird in Betrachtung der von Wiedenhöft $(2014,24)$ herausgearbeiteten Forschungslücke zugleich ihr spezifisches Verständnis von Lernen erkennbar, welches sie durch die drei Charakteristika «Lernende, Lehrende und das zu erwerbende Wissen» ausdrückt. In dieser Hervorhebung wird bereits ein spezifischer Fokus auf institutionelles Lernen mit Lehrenden (im Gegensatz zum informellen Lernen in Gemeinschaften) sowie auf den Erwerb von Wissen (im Gegensatz zum Erlernen von Fähigkeiten und Verhaltensänderungen) sichtbar.

«Metaphern von Schule» ist der Titel des Dissertationsprojektes von Geffert (2006), welches von Gansen $(2010,164)$ und Schmitt $(2017,226)$ als exemplarische Untersuchung diskutiert wird. Im Fokus der empirischen Studie von Geffert $(2006,7)$ steht die Frage, «welche Metaphern (und metaphorischen Konzepte) benachteiligte Schülerinnen und Schüler von Schule generieren» (ebd., 7). Diese Frage ist für Geffert $(2006,165)$ vor dem Hintergrund seiner Annahme interessant, dass Metaphern als «Konstruktionsprinzip zur Organisation unserer Erfahrung» verstanden werden 
können. Für seine Metaphernanalyse wurde das empirische Material von Geffert $(2006,205)$ «aus 40 Aufsätzen von Schülerinnen und Schülern sowie aus 3 Gruppendiskussionen mit insgesamt 21 Schülerinnen und Schülern gewonnen». Im Rahmen der Auswertung der Sprachbilder der Schülerinnen und Schüler stellt Geffert (2006, 208 ff.) zunächst die 12 empirisch rekonstruierten metaphorischen Konzepte vor, z. B. "Schule ist eine Versorgungsanstalt», «Schule und Lernen ist Arbeit», "Schule und Unterricht ist eine schwere Last» oder «Schule und Lernen ist ein Weg, den man gehen muss». Interessant an diesen Formulierungen ist, dass die rekonstruierten Konzepte sich in den allgemeinen metaphorischen Konzepten von Wiedenhöft (2014) nur begrenzt identifizieren lassen.

Anschliessend zeigt Geffert (2006) auf, welche (empirisch bekannten) Metaphern von den Schülerinnen und Schülern im Rahmen der jeweiligen Konzepte nicht vorkommen und welche (empirisch bekannten) Konzepte von den Schülerinnen und Schülern überhaupt nicht artikuliert werden. Auf Basis der Auseinandersetzung mit Erkenntnissen weiterer Untersuchungen als Interpretationsfolie wird es für Geffert $(2006,239)$ möglich, das jeweils Andere dieser Konzepte zu erkennen. So kann sichtbar werden, «dass die soziale Konstruktion von Wirklichkeiten aus den Metaphernfeldern Benachteiligter eine andere ist als die der Lehrerinnen und Lehrer und Personen im Lehr-Lernkontext und somit unterschiedliche, und zum Teil inkompatible, Zielstellungen hervorbringen kann». So arbeitet Geffert (2006, $238 \mathrm{ff}$.) u. a. die folgenden spannungsvollen Aspekte heraus:

- In dem metaphorischen Konzept «Schule ist eine Versorgungsanstalt» steht für Geffert (2006, 211 f.) die Versorgung der Schülerinnen und Schüler durch die Lehrerinnen und Lehrer im Vordergrund. Dabei wird in dem rekonstruierten Konzept vor allem der Einsatz der Lehrerinnen und Lehrer betont und die Metaphorik des «Bringens» und des «Gebens» der Lehrkräfte betont. Geffert $(2006,240)$ weist darauf hin, dass in diesem Konzept der «Lehr-Lern-Prozess als «Weitergabe) konzeptualisiert werden [kann]». In diesem Konzept bleibt der Lernende gleichwohl überwiegend passiv (Geffert 2006, 240). Ebenfalls denkbare, mit dem Konzept kompatible Metaphern die dem Lernenden eine aktivere Rolle einräumen, wie z. B. «Lehren und Lernen ist Tauschen» wurden jedoch nicht rekonstruiert.

- In dem metaphorischen Konzept «Schule und Lernen sind Arbeit» zeigt sich für Geffert $(2006,242)$ eine Dominanz im «Machen» und 〈Arbeiten»»: «Es wird (gemacht), (gearbeitet) und an der Zukunft oder an der Beziehung (gebaut), da «laufen> Projekte, und die Schüler 'funktionieren` wie in technischen Abläufen als ‘Maschinen`» (Geffert 2006, 242). Allerdings finden im Rahmen diese Metaphorik keine Bezüge zu eigenaktiven Tätigkeiten, welche durch Begriffe wie "produktiv», «entwerfen» oder «entwickeln» zum Ausdruck gebracht werden können. Ferner weist Geffert (2006) darauf hin, dass das «Bauen» in den Konzepten der Untersuchungsgruppe vor allem negativ konnotiert war, «z. B. indem man sich seine Möglichkeiten «verbaut` oder Scheiße 〈baut»» (Geffert 2006, 242). 
- Die in der pädagogischen Sprache gebrauchte Metaphorik vom Lehr- und Lernprozess als «Bauen, Wissen ist ein Haus» (248 f.), schulisches Lehren und Lernen als «Essen» (249 f.) sowie vom «Wachsen und Gärtner» (250 f.) werden von den Schülerinnen und Schülern nicht verwendet. Für Geffert $(2006,251)$ steht in diesen Konzepten «die Aktivität des Lerners im Zentrum». Das «Fehlen» dieser Konzepte kann nach Geffert $(2006,251)$ als Indikator dafür gesehen werden, dass diese Konzepte inkompatibel mit den Konzepten der Untersuchungsgruppe sind.

Auf Basis seiner empirischen Analyse lässt sich für Geffert $(2006,251)$ am Beispiel der benachteiligten Schülerinnen und Schüler zeigen, «dass die von ihnen gebrauchte Metaphorik ihre Passivität im schulischen Erleben betont». Diese Erkenntnis wird auch von Gansen $(2010,165)$ und Schmitt $(2017,228)$ hervorgehoben. Für die vorliegende Arbeit ist das Ergebnis u. a. deshalb interessant, weil die Ergebnisse auf inhaltlicher Ebene eine deutliche Diskrepanz zu den diskutierten Leitvorstellungen für das Lehren und Lernen in der Hochschule aufweisen, wenngleich die Untersuchungsgruppe nicht identisch ist. Methodisch bietet die Arbeit ein anschauliches Beispiel, in welcher Form Daten erhoben und wie mit der Heuristik des Hiding und Highlighting neue Erkenntnisse gewonnen werden können.

Eine weitere exemplarische empirische Untersuchung zu Metaphern des Lernens von Studierenden in der «Primarlehrpersonenausbildung» hat Ryter Krebs (2008) vorgelegt. Im Fokus der empirischen Untersuchung steht für Ryter Krebs $(2008,204)$ die systematische Analyse «von alltagssprachlichen und fachsprachlichen Metaphern, die die Studierenden spontan einsetzen, um ihr Erleben des Lernens mit der Selbstlernarchitektur zu versprachlichen» (ebd.). Der Begriff der Selbstlernarchitektur steht für einen spezifischen Ansatz in der Erwachsenenbildung. Die Analyse der studentischen Metaphern erfolgte im Rahmen eines Begleitforschungsprojektes mit dem Ziel «Muster des Denkens» zu rekonstruieren. Im Kontrast zu den zuvor skizzierten Arbeiten erfolgte die Datenerhebung «im Rahmen von individuellen Lernberatungsgesprächen» (Ryter Krebs 2008, 204). Diese Gespräche wurden in zwei Abschnitte geteilt: einen Interviewabschnitt sowie einen Beratungsabschnitt. Transkribiert und ausgewertet wurden zehn Interviews von Studierenden im zweiten Semester der «Primarlehrpersonenausbildung» (Ryter Krebs 2008, 216). Die Auswertung erfolgte wie bei den zwei zuvor skizzierten Auswertungen nach Schmitt (2003). Zudem wurden die Studierenden zum Abschluss des Gesprächs aufgefordert, «ihr Lernen mit einer Metapher zu illustrieren» (Ryter Krebs 2008, 204). Diese Metaphern wurden in einem eigenen Bereich der Analyse berücksichtigt. Im Rahmen der Auswertung widmet sich Ryter Krebs $(2008,216)$ zunächst der Frage, «welche metaphorischen Ausdrücke verwenden die Studierenden, wenn sie ihr Lernen beschreiben, und welchen Herkunftsbereichen lassen sie sich zuordnen?». Dafür erfolgte im ersten Schritt eine nach Herkunftsbereichen sortierte Zusammenstellung der rekonstruierten 
Metaphernmodelle inklusive der absoluten Häufigkeit konkreter identifizierter metaphorischer Redewendungen und Einzelmetaphern.

Ryter Krebs (2008, 219 ff.) unterscheidet im Rahmen ihrer Auswertung sieben Herkunftsbereiche, welche Studierende verwenden, um ihr Erleben des Lernens mit der Selbstlernarchitektur zu beschreiben: «Raum» (219), «Ökonomischer Herkunftsbreich» (220), «Körper» (220), «Kampf/Krieg» (221), «Technik/Forschung» (221), «Natur» (221) sowie «Last» (221). Die identifizierten Einzelmetaphern und metaphorischen Redewendungen reichen von 11 «Nennungen» für den Herkunftsbereich «Last» bis zu 203 «Nennungen» für die Herkunftsbereich «Raum». Im Rahmen der verschiedenen Herkunftsbereiche unterscheidet Ryter Krebs $(2008,219)$ weiterhin metaphorische Modelle wie z. B. «Lernen heißt, auf einem Weg (vorwärts) gehen» oder «Lernen heißt, Distanz und Nähe zu einem Zentrum suchen (finden)» (Tab. 5.2). Insgesamt unterscheiden Ryter Krebs (2008, 219 ff.) 40 verschiedene Metaphernmodelle mit 11 Modellen im Herkunftsbereich Raum und lediglich einem Modell im Herkunftsbereich «Last».

\begin{tabular}{|l|l|}
\hline Metaphernmodelle & Ankerbeispiele \\
\hline $\begin{array}{l}\text { Lernen heißt, auf einem Weg } \\
\text { vorwärts gehen (70) }\end{array}$ & $\begin{array}{l}\text { - Ich bin nicht sehr weit } \\
\text { - Sollte ich da vorwärts machen } \\
\text { - Weil ich ja nicht auf der Strecke bleiben soll } \\
\text { - Ich muss ja zum Ziel kommen } \\
\text { - Es findet halt viel so an Gedankenschritten statt }\end{array}$ \\
\hline $\begin{array}{l}\text { Lernen heißt, Distanz und } \\
\text { Nähe zu einem Zentrum su- } \\
\text { chen (finden) (27) }\end{array}$ & $\begin{array}{l}\text { - Kann ich mich ein bisschen konzentrieren } \\
\text { - Dann kann ich alles rundherum vergessen } \\
\text { - Dass mein Leben jetzt wirklich darum dreht } \\
\text { - Und habe das dann auch so ausgeweitet }\end{array}$ \\
\hline
\end{tabular}

Tab. 5.2: Auszug aus der Darstellung der Metaphernmodelle zum Herkunftsbereich Raum von Ryter Krebs (2008, 219).

Für die vorliegende Arbeit eröffnen die Ergebnisse einen weiteren Einblick, wie das Erleben und die Vorstellungen von Lernen mithilfe von Metaphern versprachlicht werden können. Die Untersuchungsgruppe ist hinsichtlich der Semesteranzahl sowie der Ausrichtung des Studiengangs vergleichbar mit den Lehramtsstudierenden der TU Darmstadt. Wenngleich die Ergebnisse von Ryter Krebs (2008) verschiedene Fragen aufgeworfen haben, eröffnet die Diskussion einzelner metaphorischer Modelle interessante Einsichten dazu, wie Studierende Formulierungen eines bestimmten Herkunftsbereichs (z. B. hinsichtlich der Weg-Metaphorik) adaptieren und so unterschiedliche Qualitäten des Lernens zum Ausdruck bringen (Ryter Krebs 2008, 226). Zudem zeigt Ryter Krebs $(2008,242$ f.) auf, wie die Kombination unterschiedlicher Metaphernmodelle «eine spezifische Deutung der persönlichen Lernerfahrung» schaffen kann. 


\subsubsection{Didaktische Funktionen von Metaphern in Bildungskontexten}

Mit der Verwendung von Metaphern in Bildungskontexten - vor allem expliziter bzw. absichtsvoller Metaphern (Beger 2016) - werden unterschiedliche Funktionen und Leistungen sowie potentielle Wirkungen diskutiert (z. B. Beger 2016; Gansen 2010; Ryter Krebs 2008). Dafür untersucht Beger (2016) den Einsatz absichtsvoller Metaphern in universitären Lehrveranstaltungen hinsichtlich unterschiedlicher Formen und Funktionen. Ryter Krebs (2008) diskutiert die Funktionen und Wirkungen von Metaphern im Kontext von Lernberatungsgesprächen und hinsichtlich der Auseinandersetzung mit Texten didaktischer Modelle und Theorien. Gansen (2010) widmet sich der Auseinandersetzung mit Funktionen und Leistungen von Metaphern in umfassender Weise. Zur Eröffnung eines strukturierten Einblicks in den pädagogischen bzw. didaktischen Diskussionsstand wird zwischen drei zentralen Funktionen unterschieden: der Erklärungsfunktion für Lehrende, der Verständnis- und Modellierungsfunktion für Lernende sowie der kommunikativen Funktion zur Verständigung von Lehrenden und Lernenden. Auf Basis dieser Gliederung ist es möglich, verschiedene Argumente und Diskussionsstränge der Debatte vorzustellen und hinsichtlich institutioneller Vermittlungssituationen zu verorten ${ }^{154}$.

Die Erklärungsfunktion umfasst Möglichkeiten für Lehrende, abstrakte Sachverhalte und Theorien über Metaphern für Lernende erklären bzw. zugänglich machen zu können. Mit den Worten von Ryter Krebs (2008, 212): «Durch einen Rückgriff auf einen der direkten Erfahrung zugänglicheren Herkunftsbereich wird ein abstrakter Zielbereich verständlich» gemacht. Als typisches Beispiel verweist Ryter Krebs (2008, 212) auf den Bereich der Physik, in dem bestimmte Metaphern bereits als konventionalisierte Fachsprache erscheinen. Formulierungen wie «schwarze Löcher» oder «fließender Strom» können dies exemplarisch veranschaulichen. Die Verwendung von (absichtsvollen) Metaphern bleibt in der konkreten Unterrichtspraxis und Hochschullehre jedoch keineswegs auf den Bereich der Physik beschränkt. Gansen (2010, 169 f.) markiert auf der Basis der empirischen Studie von Cameron (2003) für den Bereich des Unterrichts zunächst, dass in «möglichen alltäglichen Sprachhandlungen (wie Erklären [...])» metaphorische Formulierungen vorkommen. Metaphern können, so deutet Gansen $(2010,170)$ die Ergebnisse von Cameron (2003) weiter, «anscheinend dabei helfen, Inhalte besser zugänglich zu machen, verständlicher und anschaulicher zu machen». Beger (2016) zeigt in einer empirischen Untersuchung auf, wie Hochschullehrende aus den Bereichen Molekularbiologie, Evolutionspsychologie, Kernchemie, Sozialpsychologie Metaphern in absichtsvoller Weise zur Erklärung

154 Mit dieser Gliederung der Debatte wird nicht der Anspruch verfolgt, bestehende Systematisierungsund Strukturierungsvorschläge zu synthetisieren. Dafür lohnt eine Auseinandersetzung mit Gansen (2010). Ferner bleibt der Strukturierungsvorschlag auf klassische Vermittlungssituationen beschränkt. Kontexte für Lernberatungsgespräche oder die Auseinandersetzung mit Literatur werden in diesem Vorschlag ausgeblendet (Ryter Krebs 2008). 
von theoretischen Sachverhalten verwenden. Zum Abschluss ihrer Analyse kommt sie zu dem Fazit:

"In all lectures, metaphors were used deliberately in order to offer the students a presumably more familiar source domain at points when abstract concepts needed explanation. Hence, deliberate metaphors are used as a teaching tool in science education at university level» (Beger 2016, 83).

Die Verständnisfunktion umfasst Funktionen für Lernende, mithilfe von Metaphern unbekannte Themen, abstrakte Sachverhalte und Theorien zu lernen. In dieser Hinsicht kann die Verständnis- bzw. Modellierungsfunktion als Erklärungsfunktion auf Lernendenseite verstanden werden. Zugleich gehen die folgenden Ausführungen über die erste Funktionsbestimmung der Erklärungsfunktion hinaus. Für Gansen (2010, 168) bietet die Untersuchung von Cameron (2003) in diesem Kontext einen empirischen Beleg für die pädagogische Relevanz von Metaphern: «Besonders wichtig ist die Feststellung, dass Metaphern gerade beim Lernen von gänzlich Neuem und bei schwierigen Zusammenhängen eine wichtige Funktion der Modellbildung und Illustrierung erfüllen und damit gleichsam als «Übergangsbegriffe» dienen können» (Gansen 2010, 168). Mit dem Begriff der «Übergangsbegriffe» verweist Gansen (2010) u. a. auf eine Publikation von Petrie und Oshlag (1993) ${ }^{155}$, in der das Bild einer «Brückenfunktion» von Metaphern thematisiert wird. So diskutieren Petrie und Oshlag $(1993,608)$ die Frage in welcher Weise wir etwas neues Lernen können. Zur Beantwortung dieser Frage, so das Verständnis von Gansen (2010, 150), lohnt eine Auseinandersetzung mit Metaphern bzw. metaphorischem Denken,

«[...] denn diese seien für die Herstellung und Veränderung von Zusammenhängen zuständig. Metaphern hätten jenseits der pädagogisch bekannten ästhetischen Funktion, jenseits von Illustration und Gedächtnisstütze einen heuristischen und epistemischen Wert; sie hätten das Potential gänzlich neue Wissensbestände zu erarbeiten, indem sie diese vom Bekannten herleiten, also in dem sie verschiedene Sach- und Denkebenen überwinden bzw. mit einander verbinden. Etwas wirklich Neues zu lernen, bedeute immer einen Konzeptwechsel und neue Zusammenhänge herzustellen; dafür sei metaphorisches Denken als der ideale Weg - bzw. als eine 〈Brücke〉 anzusehen» (Gansen 2010, 150).

Für Gansen (2010, 150) können die Ausführungen von Petrie und Oshlag (1993) dabei so verstanden werden, dass sie «metaphorisches Denken und Verstehen als grundlegendes Lernprinzip» markieren. Dieses Verständnis erscheint anknüpfungsfähig an die «Theorie des erfahrungsbasierten Verstehens» von Gropengießer (2007),

155 Wenngleich Gansen $(2010,150)$ in seiner Arbeit nur den Namen von Hugh G. Petrie referenziert, erfolgte die Veröffentlichung in Zusammenarbeit mit Rebecca S. Oshlag. 
welcher im vergangenen Jahrzehnt vielfältig in der naturwissenschaftlichen Fachdidaktik aufgegriffen wurde. Eine grundlegende Annahme lautet, dass Verstehen in weiten Bereichen imaginativ ist bzw. weitgehend metaphorisch funktioniert. Unter den Begriff «Verstehen» fasst Gropengießer $(2007,105)$ «Denkprozesse und [...] kognitives Lernen». Metaphern sind in dieser Perspektive nicht nur Mittel und Werkzeug um Lernen zu ermöglichen, sondern es wird davon ausgegangen, dass kognitives Lernen nur in Form von Metaphern möglich ist.

Die kommunikative Funktion von Metaphern begründet sich einerseits vor dem Hintergrund der Debatte über die kognitive Metapherntheorie, wie sie von Beger (2016) konstruiert wird. Beger $(2016,63)$ weist darauf hin, dass die Annahme, jede Einzelmetapher bzw. konkrete metaphorische Redewendung lasse sich auf ein gedankliches metaphorisches Konzept zurückführen, von verschiedenen Autorinnen und Autoren kritisiert und zur Auflösung dieser Problematik eine Erweiterung des Modells der kognitiven Metapherntheorie vorgeschlagen wurde:

«In order to resolve the apparent paradox that language items can be seen as metaphorical on the linguistic level although they may not be processed as metaphors on a conceptual level, Steen $(2008,2010,2013)$ proposes a model of metaphor that adds a third dimension to the existing two-dimensional model of metaphor as a phenomenon of language and thought. Steen calls this essentially pragmatic level the communicative dimension of metaphor (2008: 221)» (Beger 2016, 63).

Das Metaphern im Unterricht auch jenseits der Erklärungsfunktion eine Rolle spielen, zeigen auch die Ergebnisse von Cameron, wie sie in Anlehnung an Gansen (2010) schon hinsichtlich der vorherigen Funktionen skizziert wurden. Dabei zeigt Gansen (2010) an den Ergebnissen von Cameron auf, dass Lehrkräfte Metaphern nicht nur zur Erklärung von neuen Inhalten verwenden, sondern auch «auf der Beziehungsebene, d.h. auch in ihrer pädagogischen Ansprache der Schüler» Gansen (2010, 169). So wird nachvollziehbar, dass die Verwendung von Metaphern nicht nur auf spezifische Erklärungsstrategien von Lehrenden oder dem individuellen Wissenserwerb von Lernenden beschränkt bleibt. Zudem wurde auch gezeigt, «dass solche Ausdrücke und bildlichen Konzepte im Unterrichtsdiskurs auch weiter tragen (sic) können, dass sie also dauerhafte Veranschaulichungs- und Erinnerungsstützen bieten können, auf die sich Schüler und Lehrer immer wieder beziehen und die wieder abrufbar, erweiterbar und veränderbar sind» (Gansen 2010, 168). Metaphern dienen in diesem Sinne als Diskussionsobjekte, die von einer Gruppe von Menschen geteilt werden. Dabei wird in der Untersuchung von Cameron auch erkennbar, so Gansen $(2010,169)$, wie anstrengend es ist, eine gemeinsame Sprachebene zu finden. 


\subsection{Zusammenfassung}

Das vorliegende Kapitel bildet einen zentralen Ausgangspunkt des entwicklungsorientierten Bildungsforschungsprojektes. Im Fokus stand zum einen die Diskussion zur aktuellen Herausforderung in den erziehungswissenschaftlichen Studienanteilen des Lehramtsstudiums. Die erfahrungsfundierten Lernvoraussetzungen der Studierenden stehen in einem Spannungsverhältnis zu den Zielstellungen aktueller Leitbilder. Zum anderen widmete sich das Kapitel den theoretischen und empirischen Grundlagen zur Gestaltung sowie zur Analyse dieses Spannungsfeldes. Im Fokus standen dafür Metaphern als Möglichkeit zur Thematisierung und Reflexion studentischer Vorstellungen.

Nach der methodischen Verortung (Kap.5.1) erfolgte die von Sesink und Reinmann (2015) beschriebene Phase der Problematisierung. Dafür erfolgte zunächst eine Diskussion des Leitbildes Lehrkräfte als Expertinnen und Experten für das Lehren und Lernen (Kap. 5.2.1) auf Basis verschiedener bildungspolitischer Papiere. Als zentrale Ziele des Lehramtsstudiums wurde der Aufbau einer pädagogischen Wissensbasis sowie die Entwicklung einer Reflexionsfähigkeit markiert (Kap. 5.2.1). Die Betrachtung der aktuellen Praxis der erziehungswissenschaftlichen Studien im Lehramtsstudium (Kap. 5.2.2) erfolgte mit zwei Schwerpunkten. Zum einen erfolgte ein Vergleich der Zielstellungen des Leitbildes mit der Studienordnung der TU Darmstadt (Kap. 5.2.2.1). Zum anderen wurden die Lernvoraussetzungen der Lehramtsstudierenden diskutiert (Kap. 5.2.2.1). Beim Vergleich der Zielstellungen der skizzierten Leitbilder wurde das bereits benannte Spannungsverhältnis zu den erfahrungsbasierten Vorstellungen und Erwartungen von Lehramtsstudierenden sichtbar. Zur Identifikation konkreter Gestaltungsmöglichkeiten erwies sich das Modell zur doppelten TheoriePraxis-Relationierung von Wildt $(2003,78)$ als hilfreich.

Im Anschluss erfolgte eine Aufarbeitung theoretischer Konstrukte zur Thematisierung und wissenschaftlichen Analyse erfahrungsfundierter Vorstellungen von Studierenden (Kap. 5.3). Dafür wurden die drei Forschungskonstrukte «Beliefs» bzw. Überzeugungen (Kap. 5.3.1), «Subjektive Theorien» (Kap. 5.3.2) und metaphorische Konzepte (Kap. 5.3.3) diskutiert. Die Diskussion erfolgte jeweils hinsichtlich der drei Aspekte: «Begriffliche Klärung und konzeptionelle Annahmen», «Anschlussstellen zu Forschungskonstrukten und methodischen Zugängen» sowie mögliche «Orientierung für die Praxis der Lehrerinnen- und Lehrerbildung». Beim Vergleich der konzeptionellen Annahmen der drei Konstrukte wurde die Entscheidung getroffen, die Auseinandersetzung mit Metaphern bzw. metaphorischen Konzepten zu vertiefen (Kap. 5.3.4). So wird davon ausgegangen, dass die Rekonstruktion metaphorischer Konzepte zum einen anschlussfähige Erkenntnisse für den Diskurs über Beliefs und Subjektive Theorien schaffen kann. Zum anderen bieten Metaphern aufgrund ihres Potenzials, implizite Vorstellungen bewusst zu machen und so zu Reflexionsprozessen anregen zu können, einen geeigneten Ansatz zum Umgang mit dem skizzierten 
Spannungsfeld zwischen erfahrungsfundierten Vorstellungen von Studierenden und dem Leitbild der reflexiven Lehrerinnen- und Lehrerbildung in den erziehungswissenschaftlichen Studien (Kap. 5.2.3).

In Kapitel 5.4 wurden Überlegungen zu Metaphern als Möglichkeiten zur Reflexion, Erhebung und Analyse erfahrungsbasierter Vorstellungen in den Fokus gerückt. In Anlehnung an Gropengießer $(2004,2007)$ wurden u. a. zentrale Annahmen und Anknüpfungspunkte zur Theorie des erfahrungsbasierten Verstehens vorgestellt (Kap. 5.4.1). Metaphern können in diesem Theoriekontext - metaphorisch formuliert - «als Fenster auf unsere Kognition» (Gropengießer 2007, 105) verstanden werden. So kann die Analyse impliziter und expliziter metaphorischer Denkfiguren Hinweise über die Vorstellungen über Lehr- und Lernprozesse geben. Neben den Möglichkeiten zur forschungsmethodischen Auswertung (Kap. 5.4.2) eröffnet die Formulierung und Diskussion von Metaphern zum Lehren und Lernen vielfältige didaktische Möglichkeiten zur Anregung von Reflexion eigener (oft unbewusster) Vorstellungen (Kap. 5.4.3). Gropengießer (2004) und de Guerrero und Villamil $(2000,2002)$ markieren beispielsweise die Möglichkeit, dass Metaphern und metaphorische Konzepte zum Lehr- und Lernprozess uns explizit bewusstmachen können, wie wir über Lehren und Lernen denken und sprechen. Zudem wird die Eignung von expliziten Metaphern als Reflexionsinstrumente für Lehrerinnen und Lehrern hervorgehoben (z. B. Marsch 2009; de Guerrero und Villamil 2002; Saban et al. 2007).

Zum Abschluss erfolgte im Teilkapitel «Metaphern in weiteren pädagogischen Kontexten» eine Betrachtung von Metaphern in weiteren pädagogischen Zusammenhängen. Dies erfolgte exemplarisch an drei gewählten Schwerpunkten. Der erste Schwerpunkt widmete sich unter der Überschrift «Metaphern beim pädagogischen Sprechen und Schreiben» (Kap. 5.5.1) verschiedenen Projekten zur Reflexion der Sprache der Erziehungswissenschaft und Pädagogik. Am Beispiel verschiedener Beiträge wurde markiert, dass Metaphern einen zentralen Bestandteil pädagogischer Sprache darstellen. So weisen verschiedene pädagogische Grundbegriffe einen metaphorischen Gehalt auf (Meyer-Drawe 1999). Auch in pädagogischen Fachtexten verwenden Autorinnen und Autoren in unterschiedlicher Weise metaphorische Ausdrücke und Redewendungen (Peyer und Künzli 1999). Die Analyse wissenschaftlicher Texte bzw. die Reflexion der eigenen Wissenschaftssprache stellt in der Pädagogik einen empirischen Zugang für Forschungsprojekte dar. Darüber hinaus werden im Kapitel «Empirische Ansätze zur Metaphernforschung» (Kap. 5.5.2) weitere forschungsmethodische Zugänge skizziert. Als Datenbasis werden u. a. öffentliche Zeitungsartikel sowie Schreibprodukte von Schülerinnen und Schülern analysiert. Der dritte Schwerpunkt umfasst das Thema: «Didaktische Funktionen von Metaphern in Bildungskontexten» (Kap. 5.5.3). Jenseits empirischer Forschungsmethoden wurden drei didaktische Funktionen von Metaphern in Vermittlungssituationen skizziert: die Erklärungsfunktion, die Verständnisfunktion sowie die kommunikative Funktion. 
Die theoretischen und empirischen Grundlagen dieses Kapitels bilden die zentralen Orientierungspunkte für die theoriegeleitete Entwicklung eines Konzepts sowie für den Entwurf konkreter Lehrveranstaltungen (Kap. 6). Auf Basis der vorgestellten Erkenntnisse und Einblicke erfolgte zudem die Konzeption des forschungsmethodischen Vorgehens zur Analyse studentischer Metaphern, welche bereits in den Überlegungen zur Datenerhebung und Auswertung (Kap. 3.3.4, 3.4.3) beschrieben wurde. Der dort gewählte Begriff der metaphorischen Muster steht für eine Bündelung einzelner metaphorischer Formulierungen von Studierenden, welche Gemeinsamkeiten hinsichtlich des konkreten Bild- bzw. Quellbereichs sowie des abstrakten Zielbereichs aufweisen. Der Begriff des metaphorischen Musters ist damit anschlussfähig an die Begriffe «metaphorisches Konzeptes» von Schmitt (2017) (Kap. 5.3.2), an den Begriff der «Denkfigur» von Gropengießer (2004) (Kap. 5.4.1) sowie an den Begriff «Metaphernmodell» von Ryter Krebs (2008). Aufgrund der von Schmitt (2017) formulierten Kritik an spezifischen Erhebungsmethoden (Kap. 5.4.2) zur Rekonstruktion metaphorischer Konzepte, wie sie partiell auch im vorliegenden Projekt verwendet werden, erscheint es mit dem Begriff des metaphorischen Musters angemessen zu sein, eine begriffliche Abgrenzung vorzunehmen ${ }^{156}$. Der Argumentation von de Guerrero und Villamil $(2000,2002)$ folgend wurde eine forschungsmethodische Vorgehensweise entwickelt, um die sprachlich zugänglichen Vorstellungen in Form von Metaphern zu erfassen und diese für die verschiedenen Seminargruppen zu metaphorischen Mustern verdichten zu können. Auf dieser Basis ist es möglich, den Anregungsgehalt der entwickelten Lehr- und Lernsituationen (Kap 6) in Form potenzieller Diskussions- und Reflexionsanlässe zur Entwicklung einer pädagogischen Artikulations- und Reflexionsfähigkeit erkennen und bewerten zu können (Kap. 7.4.3, 8.2, 10.4.3, 11.2).

\section{Literatur}

Abels, Simone. 2011. LehrerInnen als «Reflective Practitioner». Reflexionskompetenz für einen demokratieförderlichen Naturwissenschaftsunterricht. Wiesbaden: VS Verlag für Sozialwissenschaften.

Alger, Christianna L. 2009. «Secondary teachers' conceptual metaphors of teaching and learning: Changes over the career span». Teaching and Teacher Education 25 (5): 743-51. https:// doi.org/10.1016/j.tate.2008.10.004.

Appelt, Ralf. 2011. «Blogfarm an der Fakultät EPB». In Medien \& Bildung, herausgegeben von Torsten Meyer, Wey-Han Tan, Christina Schwalbe, und Ralf Appelt, 388-94. Wiesbaden: VS Verlag für Sozialwissenschaften. https://doi.org/10.1007/978-3-531-92082-5_31.

156 Das beschriebene Vorgehen zur Erhebung und Analyse von Seminarprodukten zur Artikulation expliziter Metaphern kann aufgrund der Anforderungen an die Datenmaterialien nicht den Anspruch verfolgen, auf individueller Ebene unbewusste metaphorische Konzepte zu rekonstruieren. 
Arbeitsgruppe Erziehungswissenschaft, Bardo Herzig. 1997. «Arbeitsgruppe «Erziehungswissenschaft»». In Neue Medien - neue Aufgaben für die Lehrerausbildung: Tagungsdokumentation, herausgegeben von Gerhard Tulodziecki und Sigirid Blömeke, 39-53. Gütersloh: Verlag Bertelsmann Stiftung.

Arnold, Rolf, und Hans-Georg Kempkes. 1998. «Praktisches des Konstruktivismus». Hessische Blätter für Volksbildung 48.

Arslan, Derya, und Zeynep Karatas. 2015. «Mirror of Prospective Teachers' Mind: Metaphors». Procedia - Social and Behavioral Sciences 197 (Supplement C): 1464-71. https://doi. org/10.1016/j.sbspro.2015.07.096.

Aufenanger, Stefan. 2003. «Medienkompetenz und Medienbildung». ajs-informationen: Medienkompetenz und Chancengleichheit I: 1-9.

Aydin, Selami. 2012. "A review of research on Facebook as an educational environment». Educational Technology Research and Development 60 (6): 1093-1106. https://doi.org/10.1007/ s11423-012-9260-7.

Baacke, Dieter. 1973. Kommunikation und Kompetenz. München: Juventa.

Baacke, Dieter. 1997. Medienpädagogik. Bd. 1. Grundlagen der Medienkommunikation. Tübingen: Niemeyer.

Bader, Roland. 2010. «Orientierung im virtuellen Raum - Mentale Modelle internetgestützter Lernumgebungen im Studium der Sozialen Arbeit». In Digitale Lernwelten, herausgegeben von Kai-Uwe Hugger und Markus Walber, 157-74. Wiesbaden: VS Verlag für Sozialwissenschaften. https://doi.org/10.1007/978-3-531-92365-9_10.

Barth, Matthias. 2008. «Das Lernen mit Neuen Medien als Ansatz zur Vermittlung von Gestaltungskompetenz». In Kompetenzen der Bildung für nachhaltige Entwicklung, herausgegeben von Inka Bormann und Gerhard Haan, 199-213. Wiesbaden: VS Verlag für Sozialwissenschaften. https://doi.org/10.1007/978-3-531-90832-8_14.

Baumert, Jürgen, und Mareike Kunter. 2006. «Stichwort: Professionelle Kompetenz von Lehrkräften». Zeitschrift für Erziehungswissenschaft 9 (4): 469-520. https://doi.org/10.1007/ s11618-006-0165-2.

Baumgartner, Peter. 2006. «Web 2.0: Social Software \& E-Learning». Computer + Personal (CoPers), Schwerpunktheft: E-Learning und Social Software 14 (8): 20-22.

Beger, Anke. 2016. «Different Functions of (Deliberate) Metaphor in Teaching Scientific Concepts». METAPHORIK.de 26: 61-86.

Berlanga, Adriana J., Francisco García Peñalvo, und Peter B. Sloep. 2010. «Towards eLearning 2.0 University». Interactive Learning Environments 18: 199-201.

Biermann, Ralf, Johannes Fromme, und Dan Verständig. 2014. «Partizipative Medienkulturen als Transformation von Beteiligungsmöglichkeiten - Einleitung». In Partizipative Medienkulturen: Positionen und Untersuchungen zu veränderten Formen öffentlicher Teilhabe, herausgegeben von Ralf Biermann, Johannes Fromme, und Dan Verständig, 7-17. Wiesbaden: Springer Fachmedien Wiesbaden. https://doi.org/10.1007/978-3-658-01793-4_1.

Blömeke, Sigrid. 2000. Medienpädagogische Kompetenz: theoretische und empirische Fundierung eines zentralen Elements der Lehrerausbildung. 1. Aufl. München: kopaed. 
Blömeke, Sigrid. 2004. «Empirische Befunde zur Wirksamkeit der Lehrerbildung». In Handbuch Lehrerbildung, herausgegeben von Sigrid Blömeke, Peter Reinhold, Gerhard Tulodziecki, und Johannes Wildt, 59-91. Bad Heilbrunn: Julius Klinkhard Verlag.

Blömeke, Sigrid. 2005. «Medienpädagogische Kompetenz: Theoretische Grundlagen und erste empirische Befunde». In Kompetenzdiagnostik: Theorien und Methoden zur Erfassung und Bewertung von beruflichen Kompetenzen, herausgegeben von Andreas Frey, 1. Aufl., 76-97. Landau in der Pfalz: Empirische Pädagogik.

Blömeke, Sigrid, Dana Eichler, und Christiane Müller. 2003. «Rekonstruktion kognitiver Strukturen von Lehrpersonen als Herausforderung für die empirische Unterrichtsforschung. Theoretische und methodologische Überlegungen zu Chancen und Grenzen von Videostudien». Unterrichtswissenschaft 31 (2): 103-121.

BMBF, Bundesministerium für Bildung und Forschung. 2010. Kompetenzen in einer digital geprägten Kultur. Medienbildung für die Persönlichkeitsentwicklung, für die gesellschaftliche Teilhabe und für die Entwicklung von Ausbildungs- und Erwerbsfähigkeit. https://www.dlr.de/ pt/Portaldata/45/Resources/a_dokumente/bildungsforschung/Medienbildung_Broschuere_2010.pdf.

BMBF, Bundesministerium für Bildung und Forschung. 2016. Bildungsoffensive für die digitale Wissensgesellschaft. Strategie des Bundesministeriums für Bildung und Forschung. https:// www.bmbf.de/files/Bildungsoffensive_fuer_die_digitale_Wissensgesellschaft.pdf.

BMFSFJ, Bundesministerium für Familie, Senioren, Frauen und Jugend. 2013. Medienkompetenzförderung für Kinder und Jugendliche. Eine Bestandsaufnahme. http://www.medienkompetenzbericht.de/pdf/Medienkompetenzfoerderung_fuer_Kinder_und_Jugendliche.pdf.

Bonk, Curtis J., Mimi Miyoung Lee, Nari Kim, und Meng-Fen Grace Lin. 2009. «The tensions of transformation in three cross-institutional wikibook projects». The Internet and Higher Education 12 (3-4): 126-35. http://dx.doi.org/10.1016/j.iheduc.2009.04.002.

Bos, Wilfried, Birgit Eickelmann, Julia Gerick, Frank Goldhammer, Heike Schaumburg, Knut Schwippert, Martin Senkbeil, Renate Schulz-Zander, und Heike Wendt, Hrsg. 2014. «ICILS 2013. Computer- und informationsbezogene Kompetenzen von Schülerinnen und Schülern in der 8. Jahrgangsstufe im internationalen Vergleich». Münster [u. a.]: Waxmann.

Bundestag, Deutscher. 2015. Antrag der Fraktionen der CDU/CSU und SPD: Durch Stärkung der Digitalen Bildung Medienkompetenz fördern und digitale Spaltung überwinden. Bd. Drucksache 18/4422. http://dipbt.bundestag.de/extrakt/ba/WP18/660/66045.html.

Busemannn, Katrin. 2013. «Wer nutzt was im Social Web? Ergebnisse der ARD/ZDF-Onlinestudie 2013». Media Perspektiven 7-8: 391-399.

Busemannn, Katrin, und Christoph Gscheidle. 2012. «Web 2.0: Habitualisierung der Social Communitys». Media Perspektiven 7-8: 380-390.

Cameron, Lynne. 2003. Metaphor in educational discourse. Advances in applied linguistics. London [u. a.]: Continuum.

Dalsgaard, Christian, und Morten Paulsen. 2009. «Transparency in Cooperative Online Education». The International Review of Research in Open and Distributed Learning 10 (3). http:// www.irrodl.org/index.php/irrodl/article/view/671. 
Danciu, Elena, und Gabriela Grosseck. 2011. «Social aspects of web 2.0 technologies: Teaching or teachers' challenges?» Procedia - Social and Behavioral Sciences 15: 3768 - 3773. https:// doi.org/10.1016/j.sbspro.2011.04.371.

Dander, Valentin. 2014. «Die Kunst des Reg(istr)ierens mit Big Data. Ein Versuch über Digitale Selbstverteidigung und Aktive Medienarbeit mit Daten.» medienimpulse. Beiträge zur Medienpädagogik, Nr. 4: 1-13.

Dann, Hanns-Dietrich. 1989. «Subjektive Theorien als Basis erfolgreichen Handelns von Lehrkräften». Beiträge zur Lehrerinnen- und Lehrerbildung 7 (2): 247-254.

Dittler, Ullrich, Hrsg. 2007. Online-Communities als soziale Systeme: Wikis, Weblogs und Social Software im E-Learning. Bd. 40. Medien in der Wissenschaft. Münster [u. a.]: Waxmann.

Döbeli Honegger, Beat. 2016. Mehr als 0 und 1. Schule in einer digitalisierten Welt. Bern: hep verlag.

Dohn, Nina Bonderup. 2009. «Web 2.0: Inherent tensions and evident challenges for education». International Journal of Computer-Supported Collaborative Learning 4 (3): 343-363. https:// doi.org/10.1007/s11412-009-9066-8.

Dron, Jon. 2007. «Designing the undesignable: Social software and control». Journal of Educational Technology \& Society 10 (3): 60-71.

Ebner, Martin, Conrad Lienhardt, Matthias Rohs, und Iris Meyer. 2010. «Microblogs in Higher Education - A chance to facilitate informal and process-oriented learning?» Computers \& Education 55 (1): 92-100. http://dx.doi.org/10.1016/j.compedu.2009.12.006.

Ehlers, Ulf-Daniel. 2010. «Qualität für digitale Lernwelten: Von der Kontrolle zur Partizipation und Reflexion». In Digitale Lernwelten: Konzepte, Beispiele und Perspektiven, herausgegeben von Kai-Uwe Hugger und Markus Walber, 59-73. Wiesbaden: VS Verlag für Sozialwissenschaften. https://doi.org/10.1007/978-3-531-92365-9_4.

Ehlers, Ulf-Daniel, Heimo H. Adelsberger, und Sinje Teschler. 2009. «Reflexion im Netz: Auf dem Weg zur Employability im Studium». In E-Learning 2009: Lernen im digitalen Zeitalter, herausgegeben von Nicolas Apostolopoulos, Harriet Hoffmann, Veronika Mansmann, und Andreas Schwill, 15-29. Medien in der Wissenschaft. Münster [u. a.]: Waxmann.

Faulstich, Peter. 2008. «Lernen». In Erziehungswissenschaft. Ein Grundkurs, herausgegeben von H. Faulstich-Wieland und P. Faulstich, 33-58. Reinbek bei Hamburg: Rowohlt.

Fraillon, Julian, John Ainley, Wolfram Schulz, Tim Friedman, und Eveline Gebhardt. 2014. Preparing for Life in a Digital Age: The IEA International Computer and Information Literacy Study International Report. Berlin [u. a.]: Springer. http://www.doabooks.org/ doab? func $=$ fulltext $\&$ rid $=17333$.

Fussangel, Kathrin. 2008. «Subjektive Theorien von Lehrkräften zur Kooperation : eine Analyse der Zusammenarbeit von Lehrerinnen und Lehrern in Lerngemeinschaften». PhD Thesis, Bergische Universität Wuppertal. http://nbn-resolving.de/urn:nbn:de:hbz:468-20080475.

Gansen, Peter. 2010. Metaphorisches Denken von Kindern: theoretische und empirische Studien zu einer pädagogischen Metaphorologie. Bd. 56. Erziehung Schule Gesellschaft. Würzburg: ERGON Verlag. 
Gapski, Harald. 2001. Medienkompetenz. Eine Bestandsaufnahme und Vorüberlegungen zu einem systemtheoretischen Rahmenkonzept. Wiesbaden: Westdeutscher Verlag.

Geffert, Bruno. 2006. «Metaphern von Schule : welche Metaphern und metaphorischen Konzepte generieren Benachteiligte von Schule». Schriftenreihe Studien zur Berufspädagogik. PhD Thesis, Hamburg: TU Darmstadt.

Gerstenmaier, Jochen, und Heinz Mandl. 1995. «Wissenserwerb unter konstruktivistischer Perspektive». Zeitschrift für Pädagogik 41 (6): 867-88.

GI, Gesellschaft für Informatik. 2016. Stellungnahme zum KMK-Strategiepapier «Bildung in der digitalen Welt». Gesellschaft für Informatik. https://fb-iad.gi.de/fileadmin/FB/IAD/Dokumente/gi-fbiad-stellungnahme-kmk-strategie-digitale-bildung.pdf.

GMK, Gesellschaft für Medienpädagogik und Kommunikationskultur. 2016. Stellungnahme der Gesellschaft für Medienpädagogik und Kommunikationskultur (GMK) zum Strategie-Papier der $K M K$ «Bildung in der digitalen Welt». Gesellschaft für Medienpädagogik und Kommunikationskultur. https://www.gmk-net.de/fileadmin/pdf/GMK-Stellungnahme_zum_KMK-Strategie-Entwurf.pdf.

Gouseti, Anastasia. 2010. «Web 2.0 and education: not just another case of hype, hope and disappointment?» Learning, Media and Technology 35 (3): 351-56. https://doi.org/10.1080/1743 9884.2010.509353.

Grell, Jochen, und Monika Grell. 1987. Unterrichtsrezepte. Weinheim [u. a.]: Beltz.

Grell, Petra. 2006. Forschende Lernwerkstatt. Bd. 472. Internationale Hochschulschriften. Münster [u. a.]: Waxmann.

Grell, Petra, und Franco Rau. 2011. «Partizipationslücken - Social Software in der Hochschullehre». MedienPädagogik: Zeitschrift für Theorie und Praxis der Medienbildung 21 (0): 1-23. https://doi.org/10.21240/mpaed/21/2011.11.21.X.

Grell, Petra, und Franco Rau. 2014. «Medienbildung 2.0. Soziale Online-Netzwerke in der pädagogischen Diskussion.» Computer + Unterricht 95: 14-17.

Grell, Petra, Franco Rau, und Tine Nowak. 2015. «Digitale Lehrerbildung». Verbreitung und Vernetzung von praxiserprobten Medienkonzepten zur Förderung von Medienkompetenz in der Lehrerbildung». Darmstadt: Institut für Allgemeine Pädagogik und Berufspädagogik.

Groeben, Norbert, Diethelm Wahl, Jörg Schlee, und Brigitte Scheele. 1988. Das Forschungsprogramm Subjektive Theorien : eine Einführung in die Psychologie des reflexiven Subjekts. Tübingen: Francke.

Gropengießer, Harald. 1997. «Schülervorstellungen zum Sehen.» Zeitschrift für Didaktik der Naturwissenschaften 3 (1): 71-87.

Gropengießer, Harald. 2004. «Denkfiguren im Lehr-Lernprozess». In Lehren fürs Leben : didaktische Rekonstruktion in der Biologie, herausgegeben von Harald Gropengießer, 8-24. Köln: Aulis Verlag Deubner.

Gropengießer, Harald. 2006. Wie man Vorstellungen der Lerner verstehen kann : Lebenswelten, Denkwelten, Sprechwelten. 2. aktualisierte Aufl. Bd. 4. Beiträge zur didaktischen Rekonstruktion. Oldenburg: Didaktische Zentrum (diz). 
Gropengießer, Harald. 2007. «Theorie des erfahrungsbasierten Verstehens». In Theorien in der biologiedidaktischen Forschung : ein Handbuch für Lehramtsstudenten und Doktoranden, herausgegeben von Dirk Krüger und Helmut Voigt, 105-16. Berlin, Heidelberg: Springer. http:// dx.doi.org/10.1007/978-3-540-68166-3_10.

Grosseck, Gabriela. 2009. «To use or not to use web 2.0 in higher education?» Procedia - Social and Behavioral Sciences 1 (1): 478-482. https://doi.org/10.1016/j.sbspro.2009.01.087.

Grzanna, Cindy. 2011. «Die Subjektiven Theorien von Absolventen der Wirtschaftspädagogik über ihre Berufsidentität - Ergebnisse einer explorativen Studie». In Grundlagenforschung zum Dualen System und Kompetenzentwicklung in der Lehrerbildung, herausgegeben von Uwe Faßhauer, Bärbel Fürstenau, und Eveline Wuttke, 9-20. Schriftenreihe der Sektion Berufs- und Wirtschaftspädagogik der Deutschen Gesellschaft für Erziehungswissenschaft (DGfE). Opladen ; Berlin: Budrich. https://doi.org/10.3224/86649461.

Guerrero, María C. M. de, und Olga S. Villamil. 2000. «Exploring ESL Teachers' Roles through Metaphor Analysis». TESOL Quarterly 34 (2): 341-51.

Guerrero, María C. M. de, und Olga S. Villamil. 2002. "Metaphorical conceptualizations of ESL teaching and learning». Language Teaching Research 6 (2): 95-120. https://doi. org/10.1191/1362168802/r1010a.

Guski, Alexandra. 2007. Metaphern der Pädagogik. Metaphorische Konzepte von Schule, schulischem Lernen und Lehren in pädagogischen Texten von Comenius bis zur Gegenwart. Explorationen. 53. Bern: Lang.

Guth, Sarah, und Corrado Petrucco. 2009. «Social Software and Language Acquisition». In Handbook of Research on E-Learning Methodologies for Language Acquisition, herausgegeben von R. Càssia Veiga Marriott und P. L. Torres, 424-42. New York: Information Science Reference.

Habermas, Jürgen. 1992. Faktizität und Geltung. Beiträge zur Diskurstheorie des Rechts und des demokratischen Rechtsstaats. Frankfurt am Main: Suhrkamp.

Hager, Ulrike. 2013. Metaphern in der Wissensvermittlung. Kognitive Metaphernkonzepte in Sachund Fachtexten zum Web 2.0. Hamburg: Diplomica Verlag GmbH.

Hall, David, und Simone Buzwell. 2013. «The problem of free-riding in group projects: Looking beyond social loafing as reason for non-contribution». Active Learning in Higher Education 14 (1): 37-49. https://doi.org/10.1177/1469787412467123.

Heinmann, Paul. 1962. «Didaktik als Theorie und Lehre». Die Deutsche Schule 54 (9): 407-427.

Herzig, Bardo, und Silke Grafe. 2010. «Entwicklung von Bildungsstandards für die Medienbildung - Grundlagen und Beispiele». In Jahrbuch Medienpädagogik 8: Medienkompetenz und Web 2.0, herausgegeben von Bardo Herzig, Dorothee M. Meister, Heinz Moser, und Horst Niesyto, 103-120. Wiesbaden: VS Verlag für Sozialwissenschaften. https://doi.org/10.1007/9783-531-92135-8_6.

Herzig, Bardo, Dorothee M. Meister, Heinz Moser, und Horst Niesyto, Hrsg. 2010. Jahrbuch Medienpädagogik 8. Medienkompetenz und Web 2.0. Wiesbaden: VS Verlag für Sozialwissenschaften.

Hessischer Bildungsserver. 2016. Portfolio Medienbildungskompetenz. http://medien.bildung. hessen.de/pomebiko/das_portfolio.html. 
Hollick, Danièle. 2013. «Informelles Lernen von Lehrerinnen und Lehrern im Kontext Schule und Migration : eine explorative Studie über Subjektive Theorien von Lehrerinnen und Lehrern». PhD Thesis, Kassel: Universität Kassel.

Horstkemper, Marianne. 2004. «Erziehungswissenschaftliche Ausbildung». In Handbuch Lehrerbildung, herausgegeben von Sigirid Blömeke, Peter Reinhold, Gerhard Tulodziecki, und Johannes Wildt, 461-76. Bad Heilbrunn: Julius Klinkhardt.

Iske, Stefan, und Winfried Marotzki. 2010. «Wikis: Reflexivität, Prozessualität und Partizipation». In Medienbildung in neuen Kulturräumen. Die deutschprachige und britische Diskussion, herausgegeben von Ben Bachmair, 1. Aufl., 141-151. Wiesbaden: VS Verlag für Sozialwissenschaften. https://doi.org/10.1007/978-3-531-92133-4_10.

Jäkel, Olaf. 2003. Wie Metaphern Wissen schaffen : die kognitive Metapherntheorie und ihre Anwendung in Modell-Analysen der Diskursbereiche Geistestätigkeit, Wirtschaft, Wissenschaft und Religion. Hamburg: Kovač.

Jank, Werner, und Hilbert Meyer. 2008. Didaktische Modelle. Berlin: Cornelsen.

Jenkins, Henry, Ravi Purushotma, Margaret Weigel, Katie Clinton, und Alice J. Robison. 2009. Confronting the Challenges of Participatory Culture. Cambridge, Massachusetts London, England: MIT Press. https://mitpress.mit.edu/books/confronting-challenges-participatoryculture.

Jörissen, Benjamin. 2011. «Medienbildung» - Begriffsverständnisse und Reichweiten». MedienPädagogik: Zeitschrift für Theorie und Praxis der Medienbildung 20 (0): 211-235. https://doi. org/10.21240/mpaed/20/2011.09.20.X.

Jörissen, Benjamin, und Winfried Marotzki. 2007. «Neue Bildungskulturen im Web 2.0: Artikulation, Partizipation, Syndiaktion». In Internet - Bildung - Gemeinschaft, herausgegeben von Friederike von Gross, Winfried Marotzki, und Uwe Sander, 203-25. Wiesbaden: Springer VS.

Jörissen, Benjamin, und Winfried Marotzki. 2009. Medienbildung - eine Einführung. Theorie Methoden - Analysen. UTB. 3189. Erziehungswissenschaft, Medienbildung. Bad Heilbrunn: Klinkhardt.

Junco, Reynol. 2012. «Too much face and not enough books: The relationship between multiple indices of Facebook use and academic performance». Computers in Human Behavior 28 (1): 187-98. http://dx.doi.org/10.1016/j.chb.2011.08.026.

Kalra, Mani Bhasin, und Bharati Baveja. 2012. «Teacher Thinking about Knowledge, Learning and Learners: A Metaphor Analysis». Procedia - Social and Behavioral Sciences 55 (Supplement C): 317-26. https://doi.org/10.1016/j.sbspro.2012.09.509.

Kammerl, Rudolf. 2015. Medienbildung - (k)ein Unterrichtsfach? Eine Expertise zum Stellenwert der Medienkompetenzförderung in Schulen. Hamburg: Medienanstalt Hamburg/SchleswigHolstein (MA HSH).

Kammerl, Rudolf, und Kerstin Mayrberger. 2011. «Medienpädagogik in der Lehrerinnen- und Lehrerbildung in Deutschland: Aktuelle Situation und Desiderata». Beiträge zur Lehrerinnenund Lehrerbildung 29 (2): 172-184. 
Kammerl, Rudolf, und Sandra Ostermann. 2010. Medienbildung - (k)ein Unterrichtsfach? Eine Expertise zum Stellenwert der Medienkompetenzförderung in Schulen. Hamburg: Medienanstalt Hamburg/Schleswig-Holstein (MA HSH).

Kammerl, Rudolf, und Alexander Unger. 2014. «Diskursive und produktive Praktiken in der Digitalen Kultur». In Jahrbuch Medienpädagogik 11: Diskursive und produktive Praktiken in der digitalen Kultur, herausgegeben von Rudolf Kammerl, Alexander Unger, Petra Grell, und Theo Hug, 7-12. Wiesbaden: Springer Fachmedien Wiesbaden. https://doi.org/10.1007/9783-658-06462-4_1.

Kane, Ruth, Susan Sandretto, und Chris Heath. 2002. «Telling Half the Story: A Critical Review of Research on the Teaching Beliefs and Practices of University Academics». Review of Educational Research 72 (2): 177-228. https://doi.org/10.3102/00346543072002177.

KBoM, Keine Bildung ohne Medien! 2009. Medienpädagogisches Manifest. http://www.keinebildung-ohne-medien.de/medienpaedagogisches-manifest/.

KBoM, Keine Bildung ohne Medien! 2011. Bildungspolitische Forderungen. Medienpädagogischer Kongress 2011. http://www.keine-bildung-ohne-medien.de/kongress-dokumentation/keine-bildung-ohne-medien_bildungspolitische-forderungen.pdf.

KBoM, Keine Bildung ohne Medien! 2014. Grundbildung Medien für alle pädagogischen Fachkräfte. http://www.keine-bildung-ohne-medien.de/wp-content/uploads/2014/11/Position_Grundbildung_KBoM.pdf.

Kim, Yunhwan, Michael Glassman, Mitchell Bartholomew, und Eun Hye Hur. 2013. "Creating an educational context for Open Source Intelligence: The development of Internet self-efficacy through a blogcentric course». Computers \& Education 69 (0): 332-42. http://dx.doi. org/10.1016/j.compedu.2013.07.034.

Kimpeler, Simone. 2010. «Lernen mit Online-Medien - E-Learning». In Handbuch Online-Kommunikation, herausgegeben von Wolfgang Schweiger und Klaus Beck, 364-84. Wiesbaden: VS Verlag für Sozialwissenschaften. https://doi.org/10.1007/978-3-531-92437-3_15.

King, Alison. 1993. «From Sage on the Stage to Guide on the Side». College Teaching 41 (1): 30-35. https://doi.org/10.1080/87567555.1993.9926781.

Klafki, Wolfgang. 1968. «Die didaktische Analyse». In Unterricht: Aufbau und Kritik, herausgegeben von Günther Dohmen, 48-57. Erziehung in Wissenschaft und Praxis ; 7. München: Piper.

Klafki, Wolfgang. 1991. Neue Studien zur Bildungstheorie und Didaktik: zeitgemäße Allgemeinbildung und kritisch-konstruktive Didaktik. 2., erw. Aufl. Reihe Pädagogik. Weinheim [u. a.]: Beltz.

KMK, Kultusministerkonferenz. 2000. Aufgaben von Lehrerinnen und Lehrern heute - Fachleute für das Lernen. Gemeinsame Erklärung des Präsidenten der Kultusministerkonferenz und der Vorsitzenden der Bildungs- und Lehrergewerkschaften sowie ihrer Spitzenorganisationen Deutscher Gewerkschaftsbund DGB und DBB - Beamtenbund und Tarifunion. http://www. kmk.org/fileadmin/Dateien/veroeffentlichungen_beschluesse/2000/2000_10_05-BremerErkl-Lehrerbildung.pdf.

KMK, Kultusministerkonferenz. 2004. Standards der Lehrerbildung: Bildungswissenschaften. Kultusministerkonferenz. http://www.kmk.org/fileadmin/Dateien/veroeffentlichungen_ beschluesse/2004/2004_12_16-Standards-Lehrerbildung.pdf. 
KMK, Kultusministerkonferenz. 2008. Ländergemeinsame inhaltliche Anforderungen für die Fachwissenschaften und Fachdidaktiken in der Lehrerbildung. http://www.kmk.org/fileadmin/Dateien/veroeffentlichungen_beschluesse/2008/2008_10_16-Fachprofile-Lehrerbildung.pdf.

KMK, Kultusministerkonferenz. 2012. Medienbildung in der Schule. (Beschluss der Kultusministerkonferenz vom 8. März 2012). Kultusministerkonferenz. https://www.kmk.org/fileadmin/ Dateien/veroeffentlichungen_beschluesse/2012/2012_03_08_Medienbildung.pdf.

KMK, Kultusministerkonferenz. 2014. Standards der Lehrerbildung: Bildungswissenschaften. Kultusministerkonferenz. http://www.kmk.org/fileadmin/Dateien/veroeffentlichungen_ beschluesse/2004/2004_12_16-Standards-Lehrerbildung-Bildungswissenschaften.pdf.

KMK, Kultusministerkonferenz. 2016a. Bildung in der digitalen Welt. Strategie der Kultusministerkonferenz. https://www.kmk.org/fileadmin/Dateien/veroeffentlichungen_ beschluesse/2016/2016_12_08-Bildung-in-der-digitalen-Welt.pdf.

KMK, Kultusministerkonferenz. 2016b. Ländergemeinsame inhaltlicheAnforderungen für die Fachwissenschaften und Fachdidaktiken in der Lehrerbildung. Kultusministerkonferenz. http:// www.kmk.org/fileadmin/Dateien/veroeffentlichungen_beschluesse/2008/2008_10_16Fachprofile-Lehrerbildung.pdf.

Kobak, Mevhibe, und Nazli Ruya Taskin. 2012. «Prospective Teachers' Perceptions of using Technology in Three Different Ways». Procedia - Social and Behavioral Sciences 46 (Supplement C): 3629-36. https://doi.org/10.1016/j.sbspro.2012.06.118.

Koc, Mustafa. 2013. «Student teachers' conceptions of technology: A metaphor analysis». Computers \& Education 68 (Supplement C): 1-8. https://doi.org/10.1016/j.compedu.2013.04.024.

Köhler, T., und J. Neumann. 2011. «Integration durch Offenheit. Wissensgemeinschaften in Forschung und Lehre». In Wissensgemeinschaften Digitale Medien - Öffnung und Offenheit in Forschung und Lehre, herausgegeben von T. Köhler und J. Neumann, 11-17. Münster [u. a.]: Waxmann.

Kraut, Boris. 2012. «Freie Bildung: Web 2.0-Tools als Türöffner für die Wirtschaft». In Grundfragen Multimedialen Lehrens und Lernens: von der Innovation zur Nachhaltigkeit, herausgegeben von Nicolas Apostolopoulos, Ulrike Mußmann, Wolfgang Coy, und Andreas Schwill, 275-80. Münster [u. a.]: Waxmann. http://www.gml-2012.de/tagungsband/Tagungsband_ GML2012_web.pdf.

Kron, Friedrich W., und Alivisos Sofos. 2003. Mediendidaktik : neue Medien in Lehr- und Lernprozessen: mit 6 Tabellen. Bd. 2404 : Medien-und Kommunikationswissenschaft, Pädagogik. München [u. a.]: UTB.

Kron, Friedrich W., Jutta Strandop, und Jürgens Eiko. 2014. Grundwissen Didaktik. 6. Auflage. München, Basel: Reinhardt.

Lakoff, George, und Mark Johnson. 1980. Metaphors we live by. Chicago [u. a.]: Univ. of Chicago Press.

Lakoff, George, und Mark Johnson. 2014. Leben in Metaphern: Konstruktion und Gebrauch von Sprachbildern. 8. Aufl. Systemische Horizonte. Heidelberg: Carl-Auer-Verl. 
Leavy, Aisling M., Fiona A. McSorley, und Lisa A. Boté. 2007. «An examination of what metaphor construction reveals about the evolution of preservice teachers' beliefs about teaching and learning». Teaching and Teacher Education 23 (7): 1217-33. https://doi.org/10.1016/j. tate.2006.07.016.

LKM, Länderkonferenz MedienBildung. 2015. Kompetenzorientiertes Konzept für die schulische Medienbildung. http://www.laenderkonferenz-medienbildung.de/files/Dateien_lkm/Dokumente/LKM-Positionspapier_2015.pdf.

Luehmann, April Lynn. 2008. «Using Blogging in Support of Teacher Professional Identity Development: A Case Study». Journal of the Learning Sciences 17 (3): 287-337.

Marsch, Sabine. 2009. «Metaphern des Lehrens und Lernens. Vom Denken, Reden und Handeln bei Biologielehrern». PhD Thesis, Berlin: Freie Universität Berlin.

Marsch, Sabine, und Dirk Krüger. 2007. «Vorstellungen von Biologielehrern». In Lehr- und Lernforschung in der Biologiedidaktik. Ausbildung und Professionalisierung von Lehrkräften, herausgegeben von Ute Harms und Angela Sandmann, 253-272. Innsbruck, Wien, Bozen: Studienverlag.

Martinez, Maria A., Narcis Sauleda, und Günter L. Huber. 2001. «Metaphors as blueprints of thinking about teaching and learning». Teaching and Teacher Education 17 (8): 965-77. https:// doi.org/10.1016/S0742-051X(01)00043-9.

Mayrberger, K. 2007. «Hochschuldidaktik und eLearning - eine förderliche Allianz für die Veränderung von akademischer Lehr- und Lernkultur». In Die Qualität akademischer Lehre. Zur Interdependenz von Hochschuldidaktik und Hochschulentwicklung, herausgegeben von M. Merkt und Mayrberger K., 189-215. Innsbruck: Studienverlag.

Mayrberger, Kerstin. 2010a. «Ein didaktisches Modell für partizipative eLearning-Szenarien Forschendes Lernen mit digitalen Medien gestalten». In Digitale Medien für Lehre und Forschung, Tagungsband der GMW-Jahrestagung 2010, herausgegeben von Schewa Mandel, Manuel Rutishauser, und Eva Seiler Schiedt, 363-375. Medien in der Wissenschaft. Münster [u. a.]: Waxmann.

Mayrberger, Kerstin. 2010b. «Web 2.0 in der Hochschule - Überlegungen zu einer (akademischen) Medienbildung für 〈E-Learning 2.0»». In Jahrbuch Medienpädagogik 8, herausgegeben von Bardo Herzig, Dorothee M. Meister, Heinz Moser, und Horst Niesyto, 309-28. Wiesbaden: VS Verlag für Sozialwissenschaften. https://doi.org/10.1007/978-3-531-92135-8_17.

Mayrberger, Kerstin. 2012. «Medienpädagogische Kompetenz im Wandel - Vorschlag zur Gestaltung des Übergangs in der Lehrerbildung am Beispiel mediendidaktischer Kompetenz». In Jahrbuch Medienpädagogik 9, herausgegeben von Renate Schulz-Zander, Birgit Eickelmann, Heinz Moser, Horst Niesyto, und Petra Grell, 389-412. Wiesbaden: VS Verlag für Sozialwissenschaften. https://doi.org/10.1007/978-3-531-94219-3_17.

Mayrberger, Kerstin. 2013. «Eine partizipative Mediendidaktik (nicht nur) für den Hochschulkontext?»In E-Learning zwischen Vision und Alltag. Zum Stand der Dinge, herausgegeben von C. Bremer und D. Krömker, 96-106. Münster [u. a.]: Waxmann. https://www.waxmann. com/?elD=texte\&pdf=2953Volltext.pdf\&typ=zusatztext. 
Mayrberger, Kerstin. 2014. «Partizipative Mediendidaktik. Inwiefern bedarf es im Kontext einer partizipativen Medienkultur einer spezifischen Mediendidaktik?» In Partizipative Medienkulturen: Positionen und Untersuchungen zu veränderten Formen öffentlicher Teilhabe, herausgegeben von Ralf Biermann, Johannes Fromme, und Dan Verständig, 261-282. Wiesbaden: Springer Fachmedien Wiesbaden. https://doi.org/10.1007/978-3-658-01793-4_12.

Mayrberger, Kerstin, und Heinz Moser. 2011. «Editorial: Partizipationschancen im Kulturraum Internet nutzen und gestalten: Das Beispiel Web 2.0». MedienPädagogik: Zeitschrift für Theorie und Praxis der Medienbildung 21 (0). https://doi.org/10.21240/mpaed/21/2011.10.10.X.

Mayrberger, Kerstin, Stephan Waba, und Michael Schwratz. 2013. «Social Media in der Lehrerbildung. Editorial». Herausgegeben von Kerstin Mayrberger, Stephan Waba, und Michael Schwratz. Journal für LehrerInnenbildung 13 (4): 4-5.

Mayring, Philipp. 1990. Qualitative Inhaltsanalyse : Grundlagen und Techniken. 2., überarb. Aufl. Weinheim [u. a.].

Mcloughlin, Catherine, und Mark J. W. Lee. 2007. «Social software and participatory learning: pedagogical choices with technology affordances in the Web 2.0 era", ICT: Providing choices for learners and learning». In ICT: Providing choices for learners and learning. Proceedings ascilite Singapore, herausgegeben von R.J. Atkinson, C. McBeath, S. K. A. Soong, und C. Cheers, 664-75. Singapore: Centre for Educational Development, Nanyang Technological University. http://www.ascilite.org/conferences/singapore07/procs/mcloughlin.pdf.

Meder, Norbert. 2007. «Theorie der Medienbildung. Selbstverständnis und Standortbestimmung der Medienpädagogik». In Jahrbuch Medien-Pädagogik 6: Medienpädagogik-Standortbestimmung einer erziehungswissenschaftlichen Disziplin, herausgegeben von Werner Sesink, Michael Kerres, und Heinz Moser, 55-73. Wiesbaden: VS Verlag für Sozialwissenschaften. https://doi.org/10.1007/978-3-531-90544-0_3.

Meyer, Hilbert. 2003. «Skizze eines Stufenmodells zur Analyse von Forschungskompetenz». In Forschendes Lernen: Theorie und Praxis einer professionellen LehrerInnenausbildung, herausgegeben von Alexandra Obolenski und Hilbert Meyer, 99-115. Bad Heilbrunn: Klinkhardt.

Meyer, Katrina A. 2010. «Web 2.0 research: Introduction to the special issue». The Internet and Higher Education 13 (4): 177-78. http://dx.doi.org/10.1016/j.iheduc.2010.07.004.

Meyer-Drawe, Käte. 1999. "Zum metaphorischen Gehalt von «Bildung〉 und 〈Erziehung»». Zeitschrift für Pädagogik 45 (2): 161-175.

Moser, Heinz. 2008. «Medien und Reformpädagogik». In Handbuch Medienpädagogik, herausgegeben von Uwe Sander, Friederike von Gross, und Kai-Uwe Hugger, 1. Aufl. Wiesbaden: VS Verlag für Sozialwissenschaften.

Moser, Heinz. 2010a. «Die Medienkompetenz und die «neue» erziehungswissenschaftliche Kompetenzdiskussion». In Jahrbuch Medienpädagogik 8: Medienkompetenz und Web 2.0, herausgegeben von Bardo Herzig, Dorothee M. Meister, Heinz Moser, und Horst Niesyto, 59-79. Wiesbaden: VS Verlag für Sozialwissenschaften. https://doi.org/10.1007/978-3-531-92135$8 \_4$.

Moser, Heinz. 2010b. Schule 2.0 : Medienkompetenz für den Unterricht. 1. Aufl. Bd. 20. Schulmanagement konkret. Kronach: Link. 
Moser, Heinz. 2012. «Bildungsstandards im Medienbereich». In Jahrbuch Medienpädagogik 9, herausgegeben von Renate Schulz-Zander, Birgit Eickelmann, Heinz Moser, Horst Niesyto, und Petra Grell, 249-269. Wiesbaden: VS Verlag für Sozialwissenschaften. https://doi. org/10.1007/978-3-531-94219-3_12.

Moser, Heinz, Petra Grell, und Horst Niesyto, Hrsg. 2011. Medienbildung und Medienkompetenz: Beiträge zu Schlüsselbegriffen der Medienpädagogik. München: kopaed.

Moser, Karin S. 2000. "Metaphor analysis in psychology-Method, theory, and fields of application». Forum Qualitative Sozialforschung / Forum: Qualitative Social Research 1 (2). http:// www.qualitative-research.net/index.php/fqs/article/view/1090/2388.

Müller, Karin. 2007. «Subjektive Theorien von Erzieher und Erzieherinnen zu Bildung im Kindergarten». Bildungsforschung 4 (1).

Munby, Hugh. 1987. «Metaphor and Teachers' Knowledge». Research in the Teaching of English 21 (4): 377-397.

Münderlein, Regina. 2013. «Schulkooperation zwischen Handlungslogik und Programmatik: Qualitative Fallstudie über Subjektive Theorien zur interinstitutionellen Zusammenarbeit bei Akteuren von Haupt-/Mittelschulen und Akteuren der Kinder- und Jugendarbeit, Schwerpunkt Ganztagsschule». PhD Thesis, München: Ludwig-Maximilians-Universität. http://nbnresolving.de/urn:nbn:de:bvb:19-156006.

Nespor, Jan. 1987. «The role of beliefs in the practice of teaching». Journal of Curriculum Studies 19 (4): 317-28. https://doi.org/10.1080/0022027870190403.

Niesyto, Horst. 2012. «Medienpädagogik in der Lehrerbildung in Baden-Württemberg. Konzeptionelle Überlegungen und praktische Schritte zu einer medienpädagogischen Grundbildung». In Jahrbuch Medienpädagogik 9, herausgegeben von Renate Schulz-Zander, Birgit Eickelmann, Heinz Moser, Horst Niesyto, und Petra Grell, 333-357. Wiesbaden: VS Verlag für Sozialwissenschaften. https://doi.org/10.1007/978-3-531-94219-3_15.

Obolenski, Alexandra, Hrsg. 2003. Forschendes Lernen : Theorie und Praxis einer professionellen LehrerInnenausbildung. Bad Heilbrunn/Obb.: Klinkhardt.

Öner, Diler. 2009. «Pre-service teachers' experiences with Wiki: challenges of asynchronous collaboration». In WikiSym '09: Proceedings of the 5th International Symposium on Wikis and Open Collaboration, 1-2. New York, NY, USA: ACM. http://doi.acm.org/10.1145/1641309.1641353.

Owen, Martin, Lyndsay Grant, Steve Sayers, und Keri Facer. 2006. OPENING EDUCATION: Social software and learning. Bristol: Futurelab. https://telearn.archives-ouvertes.fr/hal-00190335/ document.

Pajares, M. Frank. 1992. «Teachers' Beliefs and Educational Research: Cleaning Up a Messy Construct». Review of Educational Research 62 (3): 307-32. https://doi. org/10.3102/00346543062003307.

Papastergiou, Marina, Vassilis Gerodimos, und Panagiotis Antoniou. 2011. «Multimedia blogging in physical education: Effects on student knowledge and $\{\mathrm{ICT}\}$ self-efficacy». Computers \& Education 57 (3): 1998-2010. http://dx.doi.org/10.1016/j.compedu.2011.05.006.

Peterhans, Matthias, und Stefanie Sagl. 2011. (N)Onliner Atlas 2011. Initiative D21 e.V. und TNS Infratest. https://initiatived21.de/publikationen/nonliner-atlas-2011/. 
Petko, Dominik. 2011. «Praxisorientierte medienpädagogische Forschung: Ansätze für einen empirischen Perspektivenwechsel und eine stärkere Konvergenz von Medienpädagogik und Mediendidaktik». MedienPädagogik: Zeitschrift für Theorie und Praxis der Medienbildung 20 (0): 245-258. https://doi.org/10.21240/mpaed/20/2011.09.22.X.

Petrie, Hugh. G., und Rebecca S. Oshlag. 1993. «Metaphor and Learning». In Metaphor and Thought, herausgegeben von A. Ortony, 579-609. London [u. a.]: Cambridge University Press. https://doi.org/10.1017/CB09781139173865.027.

Peyer, Ann, und Rudolf Künzli. 1999. «Metaphern in der Didaktik». Zeitschrift für Pädagogik 45 (2): 177-194.

Pinnegar, Stefinee, Jessica Mangelson, Meredith Reed, und Shaina Groves. 2011. «Exploring preservice teachers' metaphor plotlines». Teaching and Teacher Education 27 (3): 639-47. https://doi.org/10.1016/j.tate.2010.11.002.

Poom-Valickis, Katrin, Tuuli Oder, und Madis Lepik. 2012. «Teachers' Beliefs Regarding their Professional Role: A Gardener, Lighthouse or Circus Director?» Procedia - Social and Behavioral Sciences 69 (Supplement C): 233-41. https://doi.org/10.1016/j.sbspro.2012.11.404.

Rau, Franco. 2013. «Social Software in der Hochschullehre : kritische Analyse didaktischer Szenarien». Universität Potsdam. https://publishup.uni-potsdam.de/opus4-ubp/frontdoor/index/index/year/2013/docld/6202.

Rau, Franco. 2017a. «Hochschullehre mit dem Social Web? Zur Einschätzung von Trendthemen in Bildungskontexten.» Herausgegeben von Diana Bücker. 〈Trendy, hip und cool`. Aufdem Weg zu einer innovativen Hochschule? Bielefeld: W. Bertelsmann Verl.

Rau, Franco. 2017b. «Interaktives und kollaboratives Lernen mit sozialen Medien? Spannungsfelder in der Hochschullehre». In Lehren und Lernen online: Lehr- und Lernerfahrungen im Kontext akademischer Online-Lehre, herausgegeben von Hedwig Rosa Griesehop und Edith Bauer, 131-148. Wiesbaden: Springer Fachmedien Wiesbaden. https://doi.org/10.1007/9783-658-15797-5_7.

Rau, Franco, Olaf Krey, und Thorid Rabe. 2013. «Fallstudien zum Wiki-gestützten Praktikum 〈Physikalische Schulexperimente»». In Inquiry-based Learning - Forschendes Lernen, herausgegeben von S. Bernholt, 683-685. Kiel: IPN.

Reinmann, Gabi. 2010. «Selbstorganisation auf dem Prüfstand: Das Web 2.0 und seine Grenzen(losigkeit)». In Digitale Lernwelten: Konzepte, Beispiele und Perspektiven, herausgegeben von Kai-Uwe Hugger und Markus Walber, 75-89. Wiesbaden: VS Verlag für Sozialwissenschaften. https://doi.org/10.1007/978-3-531-92365-9_5.

Reintjes, Christian. 2006. «Wie beurteilen die Ausbilder der zweiten Phase die pädagogischen Kenntnisse der Absolventen der ersten Phase?» In Kompetenzen und Kompetenzentwicklung von Lehrerinnen und Lehrern, herausgegeben von Cristina Allemann-Ghionda und Ewald Terhart, 51. Beiheft:182-198. Zeitschrift für Pädagogik. Weinheim [u. a.]: Beltz.

Roth, Heinrich. 1968. «Die Kunst der rechten Vorbereitung». In Unterricht: Aufbau und Kritik, herausgegeben von Günther Dohmen, 37-48. Erziehung in Wissenschaft und Praxis ; 7. München: Piper. 
Ruge, Wolfgang B. 2014. «Pädagogik * Medien - Eine Suchanfrage». In Perspektiven der Medienbildung, herausgegeben von Winfried Marotzki und Norbert Meder, 187-207. Wiesbaden: Springer Fachmedien Wiesbaden. https://doi.org/10.1007/978-3-658-03529-7_9.

Ryter Krebs, Barbara. 2008. «Rosinen picken` oder in einer Mine schürfen`? Metaphern des Lernens in Lernberatungsgesprächen». In Autonomie und Struktur in Selbstlernarchitekturen. Empirische Untersuchung zur Dynamik von Selbstlernprozessen, herausgegeben von Christiane Maier Reinhard und Daniel Wrana, 203-248. Beiträge der Schweizer Bildungsforschung. Opladen: Budrich UniPress.

Saban, Ahmet, Beyhan Nazli Kocbeker, und Aslihan Saban. 2007. «Prospective teachers' conceptions of teaching and learning revealed through metaphor analysis». Learning and Instruction 17 (2): 123-39. https://doi.org/10.1016/j.learninstruc.2007.01.003.

Sander, Uwe, Friederike von Gross, und Kai-Uwe Hugger, Hrsg. 2008. Handbuch Medienpädagogik. 1. Aufl. Wiesbaden: VS Verlag für Sozialwissenschaften.

Schaumburg, Heike, und Sebastian Hacke. 2010. «Medienkompetenz und ihre Sicht der empirischen Bildungsforschung». In Jahrbuch Medienpädagogik 8: Medienkompetenz und Web 2.0, herausgegeben von Bardo Herzig, Dorothee M. Meister, Heinz Moser, und Horst Niesyto, 147-161. Wiesbaden: VS Verlag für Sozialwissenschaften. https://doi.org/10.1007/978-3-53192135-8_6.

Schiefner, Mandy. 2011. "Social Software und Universitäten: eine kritische Analyse des Status quo». In Medien \& Bildung, herausgegeben von Torsten Meyer, Wey-Han Tan, Christina Schwalbe, und Ralf Appelt, 307-23. Wiesbaden: VS Verlag für Sozialwissenschaften. https:// doi.org/10.1007/978-3-531-92082-5_20.

Schiefner-Rohs, Mandy. 2012a. Kritische Informations- und Medienkompetenz Theoretisch-konzeptionelle Herleitung und empirische Betrachtungen am Beispiel der Lehrerausbildung. Internationale Hochschulschriften. Münster [u. a.]: Waxmann.

Schiefner-Rohs, Mandy. 2012b. «Verankerung von medienpädagogischer Kompetenz in der universitären Lehrerbildung». In Jahrbuch Medienpädagogik 9, herausgegeben von Renate Schulz-Zander, Birgit Eickelmann, Heinz Moser, Horst Niesyto, und Petra Grell, 359-387. Wiesbaden: VS Verlag für Sozialwissenschaften. https://doi.org/10.1007/978-3-531-942193_16.

Schiefner-Rohs, Mandy. 2013a. «Das Social Web als Erfahrungsraum für die Lehrerbildung - Medienbildung zwischen Werkzeug und Raum». journal für lehrerlnnenbildung 13 (4): 6-12.

Schiefner-Rohs, Mandy. 2013b. «Medienpädagogik. Strömungen, Forschungsfragen und Aufgaben». In Lehrbuch für Lernen und Lehren mit Technologien, herausgegeben von Martin Ebner und Sandra Schön, 1-9. Graz: L3T. http://l3t.eu/homepage/das-buch/ebook-2013/kapitel/o/ id/100/name/medienpaedagogik.

Schmidt, Jan. 2008. "Was ist neu am Social Web? Soziologische und kommunikationswissenschaftliche Grundlagen». In Kommunikation, Partizipation und Wirkungen im Social Web. Grundlagen und Methoden: Von der Gesellschaft zum Individuum, 18-40. Herbert von Halem Verlag. 
Schmidt, Jan-Hinrik, und Monika Taddicken, Hrsg. 2017. Handbuch Soziale Medien. Springer Reference Sozialwissenschaften. Wiesbaden: Springer VS.

Schmitt, Rudolf. 2003. «Methode und Subjektivität in der Systematischen Metaphernanalyse». Forum Qualitative Sozialforschung / Forum: Qualitative Social Research 4 (2): 22.

Schmitt, Rudolf. 2017. Systematische Metaphernanalyse als Methode der qualitativen Sozialforschung. Wiesbaden: Springer Fachmedien Wiesbaden. https://doi.org/10.1007/978-3-65813464-8.

Schneider, Ralf, Birgit Szczyrba, Ulrich Welbers, und Johannes Wildt. 2009. «Einleitung». Herausgegeben von R. Schneider, B. Szczyrba, U. Welbers, und J. Wildt. Wandel der Lehr- und Lernkulturen. Bielefeld: Bertelsmann.

Schorb, Bernd. 2008. "Handlungsorientierte Medienpädagogik». In Handbuch Medienpädagogik, herausgegeben von Uwe Sander, Friederike von Gross, und Kai-Uwe Hugger, 1. Aufl., 75-86. Wiesbaden: VS Verlag für Sozialwissenschaften.

Schulmeister, Rolf. 2009. Gibt es eine »Net Generation«? Erweiterte Version 3.0. Universität Hamburg, Zentrum für Hochschul- und Weiterbildung. http://epub.sub.uni-hamburg.de/epub/ volltexte/2013/19651/pdf/schulmeister_net_generation_v3.pdf.

Schulz, Wolfgang. 2006. «Die lehrtheoretische Didaktik». In Didaktische Theorien, herausgegeben von Herbert Gudjons und Rainer Winkel, 12., 35-56. Hamburg: Bergmann und Helbig.

Schulz-Zander, Renate, und Gerhard Tulodziecki. 2011. «Pädagogische Grundlagen für das Online-Lernen.» In Online-Lernen. Handbuch für Wissenschaft und Praxis, herausgegeben von P. Klimsa und L. J. Issing, 35-45. München: Oldenburg wissenschaftsverlag.

Selwyn, Neil, und Eve Stirling. 2016. «Social media and education ... now the dust has settled». Learning, Media and Technology 41 (1): 1-5. https://doi.org/10.1080/17439884.2015.1115769.

Sesink, Werner. 2007. «Bildung und Medium. Bildungstheoretische Spurensuche auf dem Felde der Medienpädagogik». In Jahrbuch Medien-Pädagogik 6: Medienpädagogik - Standortbestimmung einer erziehungswissenschaftlichen Disziplin, herausgegeben von Werner Sesink, Michael Kerres, und Heinz Moser, 74-100. Wiesbaden: VS Verlag für Sozialwissenschaften. https://doi.org/10.1007/978-3-531-90544-0_4.

Sesink, Werner. 2015. Entwicklungsorientierte Bildungsforschung. Plädoyer für einen «dritten Weg» in pädagogischer Forschung. Eine Textsammlung. http://www.sesink.de/wordpress/ wp-content/uploads/2015/11/Entwicklungsorientierte-Bildungsforschung_Sesink_2015. pdf.

Sesink, Werner, und Gabi Reinmann. 2015. «Umrisse eines Strukturmodells für entwicklungsorientierte bildungswissenschaftliche Forschung». In Entwicklungsorientierte Bildungsforschung. Plädoyer für einen ‘dritten Weg〉 in pädagogischer Forschung. Eine Textsammlung, herausgegeben von Werner Sesink, 69-83. http://www.sesink.de/wordpress/wp-content/ uploads/2015/11/Entwicklungsorientierte-Bildungsforschung_Sesink_2015.pdf.

Sim, Jeffrey Wee Sing, und Khe Foon Hew. 2010. «The use of weblogs in higher education settings: A review of empirical research». Educational Research Review 5 (2): 151-163. http:// dx.doi.org/10.1016/j.edurev.2010.01.001. 
Spanhel, Dieter. 2006. Medienerziehung : Erziehungs- und Bildungsaufgaben in der Mediengesellschaft. Stuttgart: Klett-Cotta.

Spanhel, Dieter. 2007. «Zur Standortbestimmung der Medienpädagogik aus anthropologischer und bildungswissenschaftlicher Sicht». In Jahrbuch Medien-Pädagogik 6: Medienpädagogik-Standortbestimmung einer erziehungswissenschaftlichen Disziplin, herausgegeben von Werner Sesink, Michael Kerres, und Heinz Moser, 33-54. Wiesbaden: VS Verlag für Sozialwissenschaften. https://doi.org/10.1007/978-3-531-90544-0_2.

Spannagel, Chrstian. 2013. «Am Modell lernen: Produktive Nutzung digitaler Medien im Lehramtsstudium». journal für lehrerInnenbildung 13 (4): 12-16.

Spitz, Malte. 2014. Was macht ihr mit meinen Daten? Hamburg: Hoffmann und Campe.

Stein, Sabine. 2007. «Professionalisierung zwischen Schule und Hochschule. Eine empirische Studie über reflexive Lehrerbildung». PhD Thesis, Pädagogische Hochschule Freiburg.

Stoffers, Ana-Maria. 2015. «Subjektive Theorien von Informatiklehrkräften zur fachdidaktischen Strukturierung ihres Unterrichts». PhD Thesis, Oldenburg: Universität Oldenburg. http:// oops.uni-oldenburg.de/2653.

Sutter, Tilmann. 2010. «Medienkompetenz und Selbstsozialisation im Kontext Web 2.0». In Jahrbuch Medienpädagogik 8: Medienkompetenz und Web 2.0, herausgegeben von Bardo Herzig, Dorothee M. Meister, Heinz Moser, und Horst Niesyto, 41-58. Wiesbaden: VS Verlag für Sozialwissenschaften. https://doi.org/10.1007/978-3-531-92135-8_3.

Taddicken, Monika, und Jan-Hinrik Schmidt. 2017. «Entwicklung und Verbreitung sozialer Medien». In Handbuch Soziale Medien, herausgegeben von Jan-Hinrik Schmidt und Monika Taddicken, 3-22. Wiesbaden: Springer Fachmedien Wiesbaden. https://doi.org/10.1007/978-3658-03765-9_1.

Terhart, Ewald. 1999. «Sprache der Erziehungswissenschaft. Eine Einführung in den Thementeil». Zeitschrift für Pädagogik 45 (2): 155-159.

Terhart, Ewald, Hrsg. 2000. Perspektiven der Lehrerbildung in Deutschland. Abschlussbericht der von der Kultusministerkonferenz eingesetzten Kommission. Weinheim [u. a.]: Beltz.

Terhart, Ewald. 2002. «Was müssen Lehrer wissen und können? Einleitende Bemerkungen zur Tagung». In Die Lehrerbildung der Zukunft - eine Streitschrift, herausgegeben von Georg Breidenstein, Werner Helsper, und Catrin Kötters-König, 17-23. Wiesbaden: VS Verlag für Sozialwissenschaften. https://doi.org/10.1007/978-3-322-80881-3_2.

Terhart, Ewald. 2009. Didaktik : eine Einführung. Stuttgart: Reclam.

Thomas, Lynn, und Catherine Beauchamp. 2011. «Understanding new teachers’ professional identities through metaphor». Teaching and Teacher Education 27 (4): 762-69. https://doi. org/10.1016/j.tate.2010.12.007.

TU Darmstadt, Institut für Allgemeine Pädagogik und Berufspädagogik. 2006. Studienordnung Lehramt an Gymnasien Grundwissenschaften. https://www.intern.tu-darmstadt.de/media/ dezernat_ii/satzungsbeilagen/sb3_06.pdf. 
TU Darmstadt. 2009. Studienordnung Lehramt an Gymnasien Grundwissenschaften. https:// www.zfl.tu-darmstadt.de/media/zfl/studium_medien/studium_lag/studium_lag_studienordnungen/grundwissenschaften/Grundwissenschaften_LaG_Ausfuehrungsbestimmung_10-2005_SB_3-06.pdf.

Tulodziecki, Gerhard, Silke Grafe, und Bardo Herzig. 2013. Gestaltungsorientierte Bildungsforschung und Didaktik: Theorie - Empirie - Praxis. Bad Heilbrunn: Klinkhardt.

Tulodziecki, Gerhard. 1992. Medienerziehung in Schule und Unterricht. 2., neubearbeitete Auflage. Bad Heilbrunn: Klinkhardt.

Tulodziecki, Gerhard. 1997. Medien in Erziehung und Bildung: Grundlagen und Beispiele einer handlungs- und entwicklungsorientierten Medienpädagogik. 3., überarb. und erw. Aufl. Bad Heilbrunn: Klinkhardt.

Tulodziecki, Gerhard. 2010. «Standards für die Medienbildung als eine Grundlage für die empirische Erfassung von Medienkompetenz-Niveaus». In Jahrbuch Medienpädagogik 8: Medienkompetenz und Web 2.0, herausgegeben von Bardo Herzig, Dorothee M. Meister, Heinz Moser, und Horst Niesyto, 81-101. Wiesbaden: VS Verlag für Sozialwissenschaften. https:// doi.org/10.1007/978-3-531-92135-8_5.

Tulodziecki, Gerhard. 2012. «Medienpädagogische Kompetenz und Standards in der Lehrerbildung». In Jahrbuch Medienpädagogik 9, herausgegeben von Renate Schulz-Zander, Birgit Eickelmann, Heinz Moser, Horst Niesyto, und Petra Grell, 271-297. Wiesbaden: VS Verlag für Sozialwissenschaften. https://doi.org/10.1007/978-3-531-94219-3_13.

Tulodziecki, Gerhard, Silke Grafe, und Bardo Herzig. 2014. «Praxis- und theorieorientierte Entwicklung und Evaluation von Konzepten für medienpädagogisches Handeln als gestaltungsorientierte Bildungsforschung.» In Jahrbuch Medienpädagogik. Methodologie und Methoden medienpädagogischer Forschung, herausgegeben von Anja Hartung, Bernd Schorb, Horst Niesyto, Heinz Moser, und Petra Grell, 10:213-229. Jahrbuch Medienpädagogik. Wiesbaden: Springer VS.

Tulodziecki, Gerhard, Bardo Herzig, und Silke Grafe. 2010. Medienbildung in Schule und Unterricht. Grundlagen und Beispiele. Bad Heilbrunn [u. a.]: UTB / Klinkhardt.

Wagner, Rudi F. 2016. Unterricht aus Sicht der Lehrerinnen und Lehrer. Subjektive Theorien zur Unterrichtsgestaltung und ihre Veränderung durch ein Training zu neuen Unterrichtsmethoden. Bad Heilbrunn: Klinkhardt.

Waycott, Jenny, Judithe Sheard, Celia Thompson, und Rosemary Clerehan. 2013. «Making students' work visible on the social web: A blessing or a curse?» Computers \& Education 68: 86-95.

Wheeler, Steve. 2009. «Learning Space Mashups: Combining Web 2.0 Tools to Create Collaborative and Reflective Learning Spaces». Future Internet 1 (1): 3. https://doi.org/10.3390/ fi1010003.

Whitcomb, Jennie, Hilda Borko, und Dan Liston. 2008. «Why Teach?: Part II». Journal of Teacher Education 59 (4): 267-72. https://doi.org/10.1177/0022487108322199. 
White, Patrick, und Neil Selwyn. 2012. «Learning online? Educational Internet use and participation in adult learning, 2002 to 2010». Educational Review 64 (4): 451-69. https://doi.org/10.1 080/00131911.2011.626123.

Wiedenhöft, Simone D. 2014. «Vom Reinziehen, Eintrichtern und Anbohren. Eine Metaphernanalyse über die Alltagssprache des Lernens». Diplomarbeit, Magisterarbeit, Universität Bremen. http://psydok.psycharchives.de/jspui/handle/20.500.11780/635.

Wildt, Johannes. 2002. «Ein hochschuldidaktischer Blick auf Lehren und Lernen». In Neues Handbuch Hochschullehre, herausgegeben von Brigitte Berendt, Hans-Peter Voss, und Johannes Wildt, 1-10. Bonn: Raabe-Verlag.

Wildt, Johannes. 2003. «Reflexives Lernen in der Lehrerbildung - ein Mehrebenenmodell in hochschuldidaktischer Perspektive». In Forschendes Lernen: Theorie und Praxis einer professionellen LehrerInnenausbildung, herausgegeben von Alexandra Obolenski und Hilbert Meyer, 71-84. Bad Heilbrunn: Klinkhardt.

Wildt, Johannes. 2005. «Auf dem Weg zu einer Didaktik der Lehrerbildung?» Beiträge zur Lehrerinnen- und Lehrerbildung 23 (2): 183-190.

Wildt, Johannes. 2013. «Entwicklung und Potentiale der Hochschuldidaktik». In Professionalisierung der Lehre. Perspektiven formeller und informeller Entwicklung von Lehrkompetenz im Kontext der Hochschulbildung, herausgegeben von H. Matthias und J. Wildt, 27-57. Bielefeld: Bertelsmann.

Winkel, Rainer. 1997. «Die kritisch-kommunikative Didaktik». In Didaktische Theorien, herausgegeben von Herbert Gudjons und Rainer Winkel, 9. Auflage, 93-112. Hamburg: Bergmann und Helbig.

Wissenschaftsrat. 2001. Empfehlungen zur künftigen Struktur der Lehrerbildung. Bd. Drs. 5065/01. https://www.wissenschaftsrat.de/download/archiv/5065-01.pdf.

Wolf, Nina. 2013. «Subjektive Theorien zum Lerngegenstand «Nachhaltigkeit > - Bedingungen und Möglichkeiten zur Förderung eines nachhaltigen Handelns im Biologieunterricht». PhD Thesis, Dortmund: TU Dortmund. 